\title{
Xenobiotica-metabolizing enzymes in the lung of experimental animals, man and in human lung models
}

\author{
F. Oesch ${ }^{1,2} \cdot$ E. Fabian $^{3} \cdot$ Robert Landsiedel $^{3}$ (I)
}

Received: 1 August 2019 / Accepted: 15 October 2019 / Published online: 31 October 2019

(c) The Author(s) 2019

\begin{abstract}
The xenobiotic metabolism in the lung, an organ of first entry of xenobiotics into the organism, is crucial for inhaled compounds entering this organ intentionally (e.g. drugs) and unintentionally (e.g. work place and environmental compounds). Additionally, local metabolism by enzymes preferentially or exclusively occurring in the lung is important for favorable or toxic effects of xenobiotics entering the organism also by routes other than by inhalation. The data collected in this review show that generally activities of cytochromes P450 are low in the lung of all investigated species and in vitro models. Other oxidoreductases may turn out to be more important, but are largely not investigated. Phase II enzymes are generally much higher with the exception of UGT glucuronosyltransferases which are generally very low. Insofar as data are available the xenobiotic metabolism in the lung of monkeys comes closed to that in the human lung; however, very few data are available for this comparison. Second best rate the mouse and rat lung, followed by the rabbit. Of the human in vitro model primary cells in culture, such as alveolar macrophages and alveolar type II cells as well as the A549 cell line appear quite acceptable. However, (1) this generalization represents a temporary oversimplification born from the lack of more comparable data; (2) the relative suitability of individual species/models is different for different enzymes; (3) when more data become available, the conclusions derived from these comparisons quite possibly may change.
\end{abstract}

Keywords Xenobiotic metabolism · Lung · Cytochrome P450 · Phase II metabolism · Experimental animals · In vitro models

\section{Introduction}

Over the past decades it became more and more obvious that the mammalian lung-for a long time step motherly treated with respect to xenobiotic metabolism-is crucial on the one hand for xenobiotic metabolism of inhaled compounds and on the other hand with respect to xenobioticametabolizing enzymes which occur selectively or even exclusively in the lung also for compounds taken up by routes other than inhalation. Since studies on pulmonary xenobiotic metabolism directly in living humans meet with

Robert Landsiedel

robert.landsiedel@basf.com

1 Institute of Toxicology, Johannes Gutenberg-University, Obere Zahlbacherstr. 67, 55131 Mainz, Germany

2 Oesch-Tox Toxicological Consulting and Expert Opinions GmbH \& Co. KG, Rheinblick 21, Wackernheim, Germany

3 Experimental Toxicology and Ecology, GV/TB, Z470, BASF SE, Carl-Bosch-Str. 38, 67056 Ludwigshafen, Germany ethical and practical constraints, models which mirror the human pulmonary metabolism as closely as possible have become crucial for predictions of desired pharmacological and undesired toxicological effects. The main purpose of this review was to present currently available information on pulmonary metabolism in experimentally used animal species and human in vitro models in order to contribute to the possibility of a rational selection of the-according to present knowledge—-relatively best-suited surrogate system for a given problem.

As will be seen from the data collected in this review and from the summary presented in Table 11 at the end of this review, the selection of an appropriate system will be different as far as different enzymes are decisively involved in the metabolism of the compound under consideration. Since different properties of the compound in question may depend on different metabolites or on the extent of the removal of the parent compound, different systems may be required for investigating different properties even for one given compound. 
The mammalian lung is a very complex organ with many compartments/subcompartments and over 40 different cell types (Stone et al. 1992). As will become obvious to the reader by the data collected in this review, the pattern of xenobiotica-metabolizing enzymes is different in these various compartments and cell types. The majority of the currently available information apt for recognizing the relative suitability of individual experimental animal species or human in vitro models for mimicking the situation in the human lung are derived from the entire organ lung, such as lung homogenates and fractions derived from them. Such comparable data are collected in the tables of this review while more qualitative than quantitative data can be found in the text of this review.

Changes of these enzyme levels and/or activities during the process of carcinogenesis and in cancer cells as well as polymorphisms and their influence on the susceptibility to cancer have been excluded from consideration in the present review and may be the topic of a future review.

Finally two words of caution. (1) The activities of some pulmonary xenobiotica-metabolizing enzymes such as the activities of some cytochromes P450 (CYPs) are very low and accordingly difficult to measure with high precision. This may explain very large standard deviations in such cases, different values given in different studies as well as observed activities in some studies versus no activities in other studies. The numbers quoted from such studies should, therefore, be viewed with caution. (2) Many activities quoted in this review and/or presented in the tables stem from original publications in which it was not stated whether the rates given have duly been tested under ascertained conditions of linearity or whether one given result was just divided by minutes and by milligrams of protein. Hence, not all values presented may really be comparable. Thus, it would be wise to consider the numerical values quoted in this review as just indicating high, moderate, low or not observed activities. Similar considerations apply to low versus no activities, since in most "zero activity" cases the limits of detection were not given by the authors and a low activity numerically given by one author in reality may have been lower than the zero activity given by another author.

Each xenobiotica-metabolizing enzyme family/superfamily is briefly introduced at the beginning of its first occurrence in the review.

\section{Xenobiotica-metabolizing enzymes in the rat lung}

\section{Cytochromes P450 (CYP)}

Cytochromes $\mathrm{P} 450$ are the major xenobiotica-metabolizing oxidoreductases in the mammalian liver and in several extra-hepatic tissues. They act as mixed-function oxidases in most cases introducing one oxygen atom of the oxygen molecule $\mathrm{O}_{2}$ into the substrate and reducing the other oxygen atom generating water. NADPH serves as reducing cofactor.

\section{CYP and CYP reductase transcript expression}

CYP reductase Sukumaran et al. (2011) reported the presence of CYP reductase mRNA in the rat lung and circadian oscillations of its levels.

CYPs Several individual observations have been reported concerning the expression of various CYPs in the rat lung exemplary given in the following approximately in chronological order (Table 1).

Zhu et al. (1985) demonstrated the presence of CYP4A mRNA in rat lung arterial endothelial and smooth muscle cells as well as in bronchial epithelial and smooth muscle cells, type I epithelial cells and macrophages.

Hellmold et al. (1993) reported that CYP4A2 and CYP 4A8 transcripts were expressed in the rat lung.

Zeldin et al. (1996) discovered the presence of the at that time novel CYP2J3 mRNA in rat lung.

Lake et al. (2003) showed in precision-cut rat lung slices induction (up to 8.3-fold) of CYP1A1 mRNA by exposure of the slices to BNF (beta-naphthoflavone), aroclor 1254 or BP (benzo[a]pyrene). Harrigan et al. (2006) confirmed and extended these observations to an increase also observed in the slices after in vivo treatment of the donor rats with TCDD (2,3,7,8-tetrachlorodibenzo-p-dioxin) and to the observation of analogous increases in the levels of CYP1B1 mRNA.

Gate et al. (2006) reported a massive increase (26-2203fold) in the CYP1A1 and 1B1 and a marked decrease (-2.4fold) in the CYP2F2 mRNA level in the rat lung upon exposure of the animals to bitumen fumes.

Hanlon et al. (2009) showed in precision-cut rat lung slices marked increases in CYP1A1 and somewhat less CYP1B1 mRNA upon treatment of the slices with the aliphatic isothiocyanates sulforaphane and erucin. Sulforaphane led to a modest increase in CYP3A2 mRNA as well. Somewhat contrary to expectation the intact glucosinolates glucoraphanin and glucoerucin also led to increases in CYP1A1 and 1B1 mRNA in precision-cut lung slices (Abdull Razis et al. 2011).

Samuelsen et al. (2012) reported that exposure of rat lung primary alveolar type 2 cells to 2 -hydroxyethyl-methacrylate (HEMA) led to nuclear translocation of the AhR (aryl hydrocarbon receptor) and to induction of CYP1A1 and CYP1B1 (and GSTA2) mRNA, known to be AhR-dependent, but not to an increase in CYP2B1 or CYP2E1 mRNA, known not to be AhR-dependent. At the same time, the cells started to have an apoptotic-like morphology, which was prevented by 
Table 1 Expression of the major xenobiotica-metabolizing human CYP1, CYP2 and CYP3 families on the mRNA level: lung contrasted with liver

\begin{tabular}{|c|c|c|}
\hline Enzyme & Lung & Liver \\
\hline CYP1A1 & $\begin{array}{l}+/-^{\mathrm{a}} \\
+++^{\mathrm{b}}\end{array}$ & ++ \\
\hline CYP1A2 & $+/-$ & +++ \\
\hline CYP1B1 & $++/+$ & + \\
\hline CYP2A6 & $++/+$ & +++ \\
\hline CYP2A13 & $+/+++$ & +++ \\
\hline CYP2B6/7 & +++ & +++ \\
\hline CYP2C8 & $+/-$ & +++ \\
\hline CYP2C9 & $+/-$ & +++ \\
\hline CYP2C18 & + & + \\
\hline CYP2C19 & $+/-$ & +++ \\
\hline CYP2D6 & $+/-$ & +++ \\
\hline CYP2E1 & $+++/++/+$ & +++ \\
\hline CYP2F1 & $+++/++$ & - \\
\hline CYP2J2 & + & ++ \\
\hline CYP2R1 & + & + \\
\hline CYP2S1 & + & - \\
\hline CYP2W1 & $++^{\mathrm{c}}$ & $+^{\mathrm{c}}$ \\
\hline CYP3A4 & $+/-$ & +++ \\
\hline CYP3A5 & $+++/++$ & $+++/++$ \\
\hline CYP3A7 & $+/-$ & + \\
\hline CYP3А43 & $+/-$ & +++ \\
\hline CYP4B1 & + & - \\
\hline
\end{tabular}

Data from 22, 58, 59, 133, 146, 151, 189, 190, 232 quoted in Pavek and Dvorak (2008); in addition: Zhu et al. (2006), Thum et al. (2006), Gervot et al. (1999), Macé et al. (1998), Raunio et al. (1999) and Nhamburo et al. (1989)

+++ , high; ++, moderate; + , low; - , undetectable; +/-, controversial

Contrasts in bold letters/symbols

${ }^{\text {a Nonsmokers }}$

${ }^{\mathrm{b}}$ Smokers

${ }^{c}$ Late-term fetal lung

co-treatment with the CYP inhibitor disulfiram. The authors suggest that HEMA leads to induction of AhR-dependent xenobiotic-metabolizing enzymes, followed by metabolism of HEMA to a reactive metabolite, which induces apoptosis.

Chan et al. (2013) reported transcript expression of CYP1A1 and CYP1B1 in the neonatal (7 days old) and adult (8 weeks old) male Sprague-Dawley rat lung. CYP1A1 and CYP1B1 mRNA were higher in the adult compared with the neonatal rat lung (CYP1A1 mRNA more than 250-fold). In the neonatal lung CYP1A1 mRNA was equally distributed between airways and lung parenchyma, but in the adult CYP1A1 mRNA was 3-fold higher in lung parenchyma while CYP1B1 mRNA levels were higher in the airways than in the alveolar region. In rat lung slices Chang et al.
(2006) showed that Clara (Club) cells (non-ciliated bronchiolar epithelial cells) were highly sensitive (and much more sensitive than other cell types) to the CYP1A1 and CYP1B1 mRNA (and protein) inducing effect of TCDD (2,3,7,8-tetrachlorodibenzo-p-dioxin) and BP.

\section{Expression of CYP and related proteins}

CYP reductase Dees et al. (1980) observed CYP reductase protein in the bronchial and bronchiolar epithelia and alveolar wall of rat lung, indistinguishable between phenobarbital-treated and control rats. Baron and Voigt (1990) confirmed and extended these findings showing the presence of CYP reductase in rat lung bronchial as well as Clara (Club) cells and ciliated bronchiolar epithelia and in type II pneumocytes, quantitatively mostly (about 2 -fold more) in the Clara cells compared with the other CYP reductasecontaining cells. The relative distribution of CYP reductase remained unchanged after treatment of the rats with 3-methylcholanthrene or Aroclor 1254.

CYPs In untreated rats CYP1A1 protein (called P450c in the original publication) was seen in only a few Clara (Club) cells (non-ciliated bronchiolar epithelial cells) and type II pneumocytes. After treatment of the rats with 3-methylcholanthrene, staining was markedly enhanced and was present in many Clara cells and type II pneumocytes and was additionally present in arteries, arterioles, veins, venules and capillaries endothelia (Keith et al. 1987). In contrast, Foster et al. (1986) observed (in untreated and in beta-naphthoflavone-treated rats) CYP1A1 protein only in bronchiolar Clara cells. The differences in the observations may have been due to differences in the methodologies used by the two teams, which include the following: Keith et al. (1987) used polyclonal antibodies raised against CYP1A1 (and polyclonal antibodies raised against CYP2B2) and fixed, embedded sections. Foster et al. (1986) used monoclonal antibodies raised against CYP1A1 and frozen sections. Baron and Voigt (1990) confirmed dramatic increases of rat lung CYP1A1 (called P-450 BNF-B and P-450 MC-B in the original publication) upon pretreatment of the rats with CYP1A1 inducers (they used 3-methylcholanthrene and Aroclor 1254). The induced protein was localized to Clara cells and to alveolar wall cells (especially type II pneumocytes), while no increase of the immunochemical staining was observed in bronchial epithelium or in ciliated bronchiolar cells. The broader spectrum CYP inducer Aroclor 1254 led to similar increases of CYP1A1 protein as observed after pretreatment of the rats with 3-methylcholanthrene, but in addition in about $30 \%$ of the cells in the bronchial epithelium. In rat lung slices Chang et al. (2006) showed that Clara cells were highly sensitive (and much more sensitive than other cell types) to the CYP1A1 and CYP1B1 protein 
(and mRNA) inducing effect of TCDD and benzo[a]pyrene. For an overview of the localization of various xenobioticametabolizing enzymes, see Table 2.

Adams et al. (1985) observed in the rat lung suppression of CYP1A1 protein synthesis after pretreatment of the animals with phenobarbital.

Somewhat in line with the very low levels of CYP1A1 protein in the rat lung described in the above-mentioned studies, Hellmold et al. (1993) did not observe any CYP1A1 protein in lung microsomes derived from untreated rats, but did observe it in those derived from BNF-treated rats.

$\mathrm{Ma}$ et al. (2002) reported a marked increase in the CYP1A1 (but not CYP2B1) protein levels in the rat lung upon intratracheal instillation of asphalt fume condensate.

Lake et al. (2003) showed in precision-cut rat lung slices induction of CYP1A1 protein by BNF or aroclor 1254. Harrigan et al. (2006) confirmed induction of CYP1A1 protein levels in precision-cut rat lung slices by treatment of the slices with BP and extended the observation to an increase also observed in the slices after in vivo treatment of the donor rats with TCDD and to the observation of analogous increases in the levels of CYP1B1 protein. Abdull Razis et al. (2011) observed increases of the CYP1A1 and 1B1 protein upon treatment of rat lung precision-cut slices with the glucosinolates glucoraphanin or glucoerucin.

Chan et al. (2013) reported protein expression of CYP1A1 and CYP1B1 in the neonatal and adult rat lung. CYP1A1 protein was increased by particulate matter ("PM") using an ultrafine premixed flame particle ("PFP") as a defined and replicable agent. The increase was transient in the adult rat lung (visible at $2 \mathrm{~h}$ but no longer at $24 \mathrm{~h}$ after an exposure of $6 \mathrm{~h}$ ). In the neonate rat lung the increase was confined to the bronchiolar epithelium, also transient, but delayed (visible at $24 \mathrm{~h}$ but no longer at $48 \mathrm{~h}$ after the $6 \mathrm{~h}$ exposure) and much weaker as compared with the adult rat lung.

CYP2B1 ("P450 PB-B") protein levels in the untreated rat lung are much greater than those of CYP1A1 ("P-450 BNF-F") (Guengerich et al. 1982; Guengerich 1990; Keith et al. 1987). Out of seven investigated organs, De Waziers et al. (1990) observed the highest concentration of the CYP2B1/2B2 protein in the rat lung, similar to duodenum, higher than in the liver. Keith et al. (1987) observed

Table 2 Localization of some xenobiotica-metabolizing enzymes proteins in the human lung. Adapted and extended from Gundert-Remy et al. (2014)

\begin{tabular}{|c|c|c|c|c|c|c|c|c|}
\hline Enzyme & $\begin{array}{l}\text { Bronchiolar } \\
\text { epithelium }^{\text {a }}\end{array}$ & $\begin{array}{l}\text { Ciliated } \\
\text { columnar epi- } \\
\text { thelial cells }\end{array}$ & $\begin{array}{l}\text { Cuboidal } \\
\text { bronchiolar } \\
\text { epithelial } \\
\text { cells }\end{array}$ & Clara cells & $\begin{array}{l}\text { Alveolar } \\
\text { epithelium }^{\text {b }}\end{array}$ & $\begin{array}{l}\text { Type I pneu- } \\
\text { mocytes }\end{array}$ & $\begin{array}{l}\text { Type II pneu- } \\
\text { mocytes }\end{array}$ & $\begin{array}{l}\text { Alveolar mac- } \\
\text { rophages }\end{array}$ \\
\hline CYP & $\begin{array}{l}\text { 1A1, 2A6, } \\
\text { 2A13, 2B6, } \\
\text { 2C } 8,2 \mathrm{C} 18, \\
2 \mathrm{E} 1,2 \mathrm{~J} 2, \\
3 \mathrm{~A} 5\end{array}$ & $1 \mathrm{~A} 1,1 \mathrm{~A} 2,2 \mathrm{~J}$ & $1 \mathrm{~A}, 3 \mathrm{~A} 5$ & $2 \mathrm{~B} 6,(2 \mathrm{~B} 7)^{\mathrm{c}}$ & $\begin{array}{l}1 \mathrm{~A} 1,2 \mathrm{~B} 6 \\
2 \mathrm{E} 1,2 \mathrm{~J} 2 \\
3 \mathrm{~A} 5\end{array}$ & $\begin{array}{l}\text { 1A1, 1A2, } \\
3 \mathrm{~A} 5\end{array}$ & $\begin{array}{l}\text { 1A1, 1A2, } \\
3 \mathrm{~A} 5\end{array}$ & $\begin{array}{l}\text { 1B1, 2E1, 2J, } \\
3 \mathrm{~A} 5\end{array}$ \\
\hline $\begin{array}{l}\text { Microsomal epox- } \\
\text { ide hydrolase }\end{array}$ & + & & & + & + & & + & + \\
\hline $\begin{array}{l}\text { NADH/NADPH } \\
\text { quinone oxidore- } \\
\text { ductase }\end{array}$ & + & & & & & & & + \\
\hline $\begin{array}{l}\text { Glutathione } \\
S \text {-transferase }\end{array}$ & + & + & & + & + & & & + \\
\hline $\begin{array}{l}\text { Phenol } \\
\text { sulfotransferase }^{\mathrm{d}}\end{array}$ & + & & & & & & & + \\
\hline $\begin{array}{l}\text { Thermolabile } \\
\text { sulfotransferase }^{\mathrm{d}}\end{array}$ & + & & & & & & & + \\
\hline $\begin{array}{l}\text { Glucuronosyl } \\
\text { transferase }^{\mathrm{d}}\end{array}$ & - & & & & & & & - \\
\hline
\end{tabular}

+ , clearly demonstrated; -, investigated and not found; no sign, not investigated

${ }^{a}$ Not differentiated between cell types

${ }^{\mathrm{b}}$ Not differentiated between type I and type II pneumocytes

${ }^{\mathrm{c}}$ Apparently one single report

${ }^{\mathrm{d}}$ Studies performed before the advent of modern nomenclature. Phenol sulfotransferases generate sulfates from phenolic compounds including phenolic and catechol drugs and neurotransmitters. One phenol-sulfotransferase sub-form is thermolabile, generates sulfate formation from micromolar concentrations of dopamine and other phenolic monoamines, and is relatively resistant to inhibition by DCNP (2,6-dichloro-4-nitrophenol) (the other sub-form is thermostable, catalyzes sulfate formation from micromolar concentrations of simple phenols such as $p$-nitrophenol, and is relatively sensitive to inhibition by DCNP). The simple term "phenol-sulfotransferase" does not distinguish between these two 
(in untreated rats and after treatment with phenobarbital or 3-methylcholanthrene) CYP2B1 in bronchiolar Clara (Club) cells and type II pneumocytes, but not in other rat lung cells. Baron and Voigt (1990) observed CYP2B1 protein in the same cell types as reported by Keith et al. (1987) and in addition in bronchial epithelial cells and in ciliated bronchiolar cells. CYP2B1 levels in Clara cells were about 2-fold higher compared with the other cell types. Using image analysis of the immunochemical determinations the relative levels were determined to be (mean integrated absorbance $\times 100$ ) as follows: Clara cells 42.7 , type II pneumocytes 30.2 , bronchial epithelial cells 29.5 , type I pneumocytes/capillary endothelial cells 23.2, ciliated bronchiolar cells 20.7. The relative distribution of CYP2B1 remained unchanged after treatment of the rats with 3-methylcholanthrene or Aroclor 1254. Cassel et al. (2000) showed preferential expression of CYP2B1 protein in rat lung Clara cells starting on postnatal day 4 in line with a C/EBP binding site in the CYP2B1 proximal promoter and with a relatively high-level expression of $\mathrm{C} /$ $\mathrm{EBP} \alpha$ and C/EBP $\delta$ in rat lung Clara cells.

Using immunochemical methods De Waziers et al. (1990) observed CYP2E1 protein in rat lung. However, levels were much lower (only $1.5 \%$ ) than in the rat liver.

CYP3A2 (called P-450 PCN-E in the original publication) protein was observed in comparable levels in untreated rat lung bronchial epithelia, Clara cells as well as ciliated bronchiolar cells, type II and other alveolar wall cells (Baron and Voigt 1990). This distribution is similar to that of CYP1A1, but dissimilar to the selective locations of CYP2B2 and CYP reductase presented above. These divergent locations are expected to lead to corresponding selective targets of toxicities of xenobiotics according to the individual locations of the CYPs responsible for their toxication. The relative distribution of CYP3A2 remained unchanged after treatment of the rats with 3-methylcholanthrene or Aroclor 1254 (Baron and Voigt 1990).

Zhu et al. (1985) demonstrated the presence of CYP4A protein in rat pulmonary arteries, bronchi and also in cultured rat lung endothelial cells.

Zeldin et al. (1996) observed CYP2J3 protein not only in rat lung tissues primarily in ciliated epithelial airway cells, but also in nonciliated epithelial airway cells, lung vascular endothelium and alveolar macrophages.

\section{CYP catalytic activities (Table 3)}

In a pioneering work Wattenberg and Leong (1962) observed benzo[a]pyrene hydroxylase [later called aryl hydrocarbon hydroxylase $(\mathrm{AHH})$ ] activity in the rat lung alveolar wall, while they did not detect the activity in bronchus or bronchioles. This lack may have been due to technical difficulties, since later Baron and Voigt (1990) clearly observed benzo[a]pyrene hydroxylation at similar levels in rat bronchi, bronchioles as well as in type II pneumocytes (and much less in other alveolar wall cells). Furthermore, Grasso et al. (1971) had detected aniline hydroxylase activity in rat bronchial and alveolar wall epithelial cells. AHH activity was dramatically increased in rat lung upon pretreatment of the rats with 3-methylcholanthrene or Aroclor 1254 (Baron and Voigt 1990). The sites of the increased AHH activity did not completely coincide with the sites of increases of CYP1A1, in line with the fact that benzo[a]pyrene is hydroxylated mainly but not exclusively by CYP1A1. Thus, AHH activity was markedly increased not only in sites where CYP 1A1 was increased by the treatment with 3-methylcholanthrene or Aroclor 1254, but in addition also in ciliated epithelial bronchiolar cells and in all bronchial epithelial cells, not only in those in which CYP1A1 was induced by Aroclor 1254.

Also very early on Clara (Club) cells were identified as a prime location for CYP-dependent covalent binding of the rodent and cattle pneumotoxin 4-ipomeanol and as prime location of its toxicity (Boyd 1977). On the same line Belinsky et al. (1987) and Devereux et al. (1988) observed that guanine O6-methylation produced by 4-( $N$-methyl- $N$ nitrosamino)-1-(3-pyridinyl)-1-butanone (NNK) was found in several cell types of the rat lung (type II pneumocytes, endothelial cells, alveolar macrophages), but quantitatively mostly in Clara cells. However, in the same studies O6-methylguanine formation upon the treatment of rats with $N$-nitrosodimethylamine was similar in rat lung type II pneumocytes, endothelial cells, alveolar macrophages as in Clara cells in line with the assumption of variously localized CYPs being chiefly responsible for the metabolic toxication of these different nitrosamines.

Lorenz et al. (1979) already very early observed considerable activities of 7-ethoxycoumarin O-deethylase in rat lung (Table 1). Rates in rat lung microsomes were 57-fold higher as compared with human lung microsomes (Oesch et al. 1980). Rates were much higher in rat Clara cells compared with rat alveolar type II cells (Devereux et al. 1989).

Bond (1983) observed in rat lung S9 significant activity for metabolizing 1-nitropyrene to 3-, 6- and 9-hydroxypyrene. The rate $(60 \mathrm{pmol} / \mathrm{min} / \mathrm{mg} \mathrm{S} 9$ protein) was considerably lower compared with the nasal and liver tissue (650 and $300 \mathrm{nmol} / \mathrm{min} / \mathrm{mg} \mathrm{S} 9$ protein, respectively). In the isolated perfused rat lung Bond and Mauderly (1984) observed biotransformation of 1-nitropyrene to 3-, 6- and 8-hydroxynitropyrene and small quantities of 10-hydroxynitropyrene. Pretreatment of the rats with 3-methylcholanthrene led to a 10 -fold increase in the total metabolism of 1-nitropyrene in the perfused lung. Pre-exposure of the rats to diesel exhaust (7400 $\mu \mathrm{g}$ particles $/ \mathrm{m}^{3}$ for 4 weeks) led to a 2-fold increase of 1-nitropyrene metabolism and to a 4-fold increase of its covalent binding to lung constituents (Bond et al. 1985).

Wheeler et al. (1990) characterized in human and baboon lung a CYP homologous to the toxicologically 


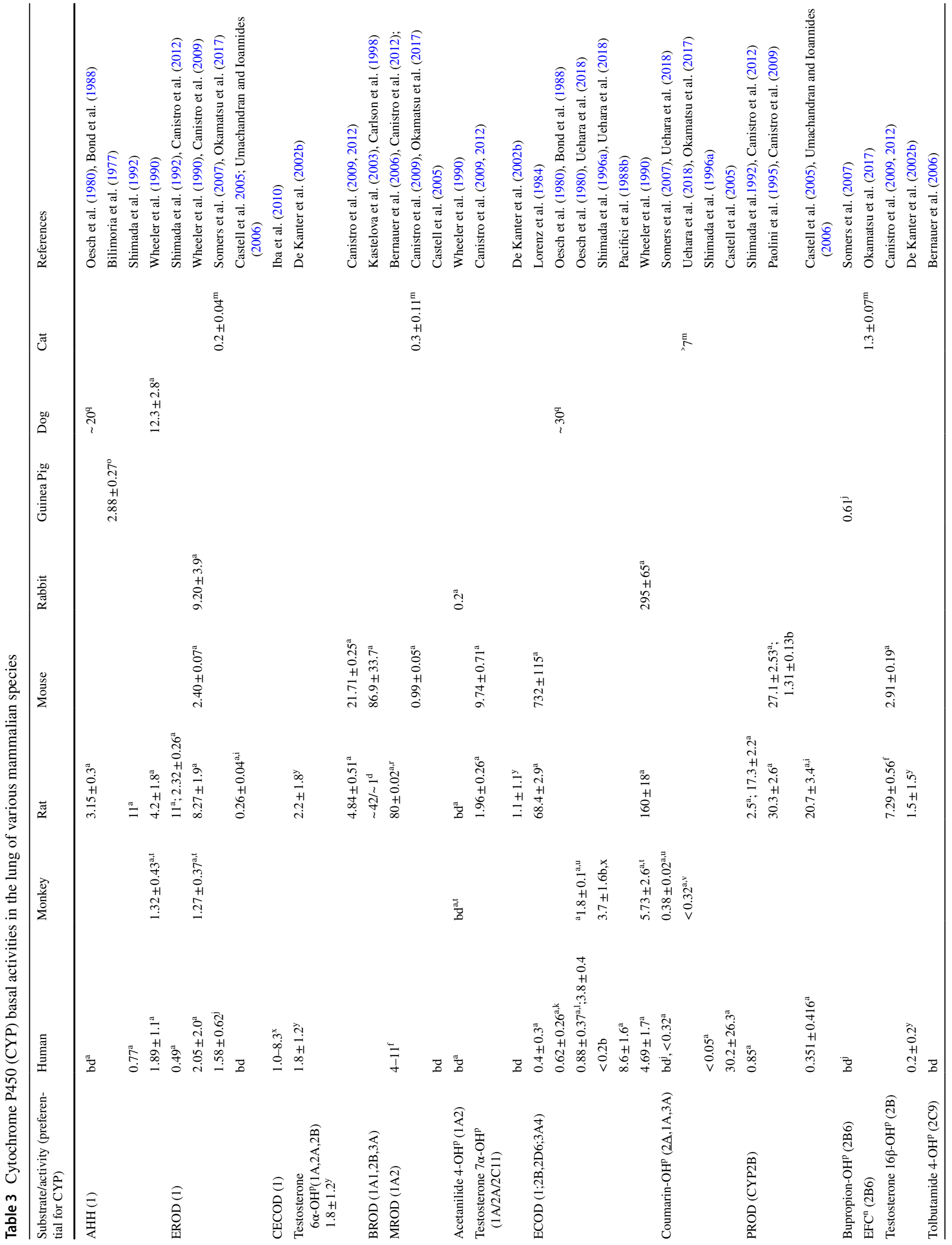




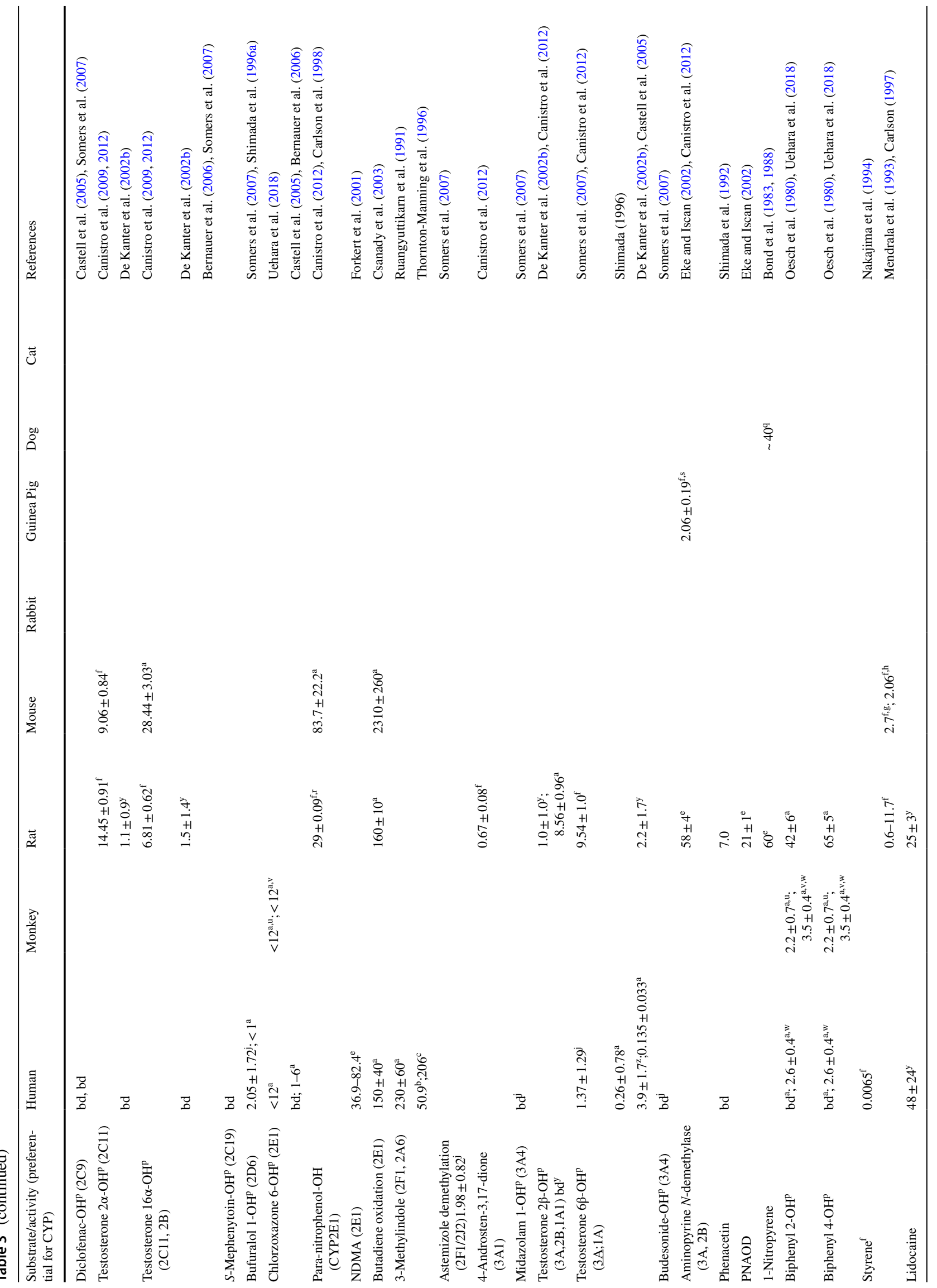




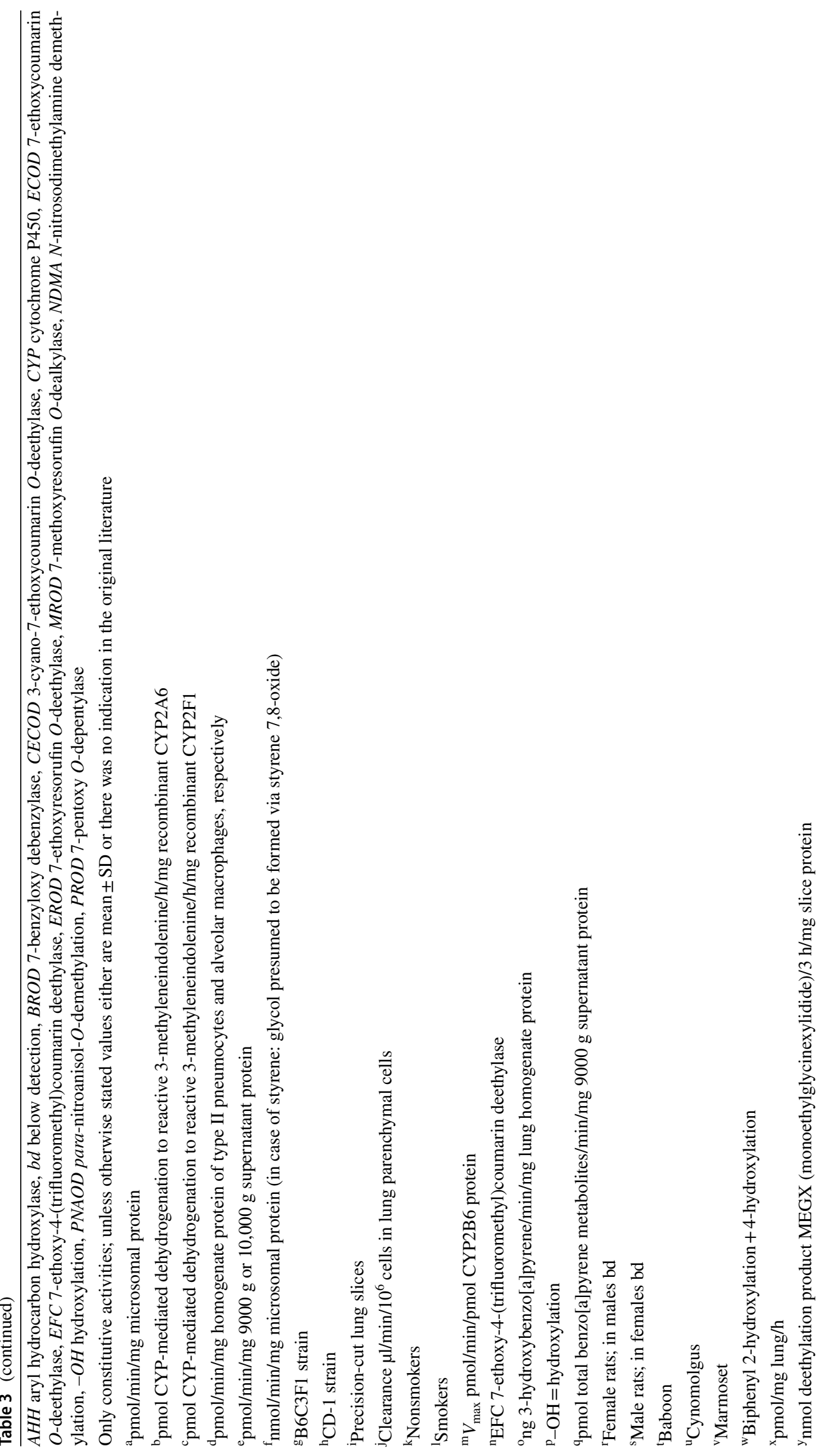


important CYP1A1 of rodents. Typical CYP1A enzymatic activities were found in human and baboon lung microsomes, in similar activities between these two species, but considerably lower activities as compared with rat and rabbit microsomes (see Table 1 comparing the Wheeler et al. 1990 data between these species).

De Waziers et al. (1990) observed relatively high constitutive 7-pentoxyresorufin $O$-depentylase (PROD) activities in the rat lung, out of seven investigated organs highest in lung and duodenum. The PROD activities in these seven organs closely correlated with the relative levels of constitutive CYP2B1/2B2 protein.

The rodent carcinogen butadiene was converted more than 10-fold slower to the monoepoxide by lung microsomes of the much less sensitive species rat compared with lung microsomes of the much more sensitive species mouse (Csanády et al. 1992).

Seaton et al. (1996) observed that isolated bronchioles of rats metabolized butadiene to the reactive butadiene epoxide only half as fast as the mice, which upon exposure to butadiene develop brochiolar-alveolar neoplasms, while rats are much less susceptible.

Shimada et al. (1992) reported the presence of AHH, 7-ethoxyreorufin $O$-deethylase (EROD) and PROD activities in rat lung microsomes which were increased by factors of 7 (AHH) and 5 (EROD) after treatment of the rats with $\beta$-naphthoflavone. Hellmold et al. (1993) observed in rat lung microsomes EROD activity which was about 100 -fold increased after pretreatment of the rats with $\beta$-naphthoflavone. They also observed in rat lung microsomes metabolic activation of the aromatic amines IQ (2-amino-3,8-dimethylimidazo[4,5-f]quinoline) and MeIQx (2-amino-3,8-dimethylimidazo[4,5-f]quinoxaline) to derivatives mutagenic for Salmonella typhimurium TA98 which they showed for IQ by inhibition studies using antibodies to be due to about $70 \%$ to CYP2A3. $\alpha$-Naphthoflavone, reputed to be a specific inhibitor of CYP1A activity, inhibited the activation of IQ by $30-40 \%$ and the activation of MeIQx by about $60-80 \%$.

Ma et al. (2002) reported an about 2.5-fold increase in EROD (but not PROD) activities in the rat lung microsomes upon intratracheal instillation of asphalt fume condensate.

Verschoyle et al. (1993) provided suggestive evidence for the importance of rat lung CYP4B1 catalytic activity for the toxication of 4-ipomeanol by observing inhibition of its covalent binding to rat lung microsomal protein by simultaneous treatment with the CYP4B1-selective substrate $p$-xylene (Smith et al. 1982).

Paolini et al. (1995) reported the presence of CYP2Bdependent PROD activity in the rat lung and its inducibility by quite a number of compounds (sodium phenobarbital, phorone, 1,2-dichloroethylene, cyclophosphamide).
Price et al. (1995) optimized conditions for using rat lung slices in xenobiotic metabolism studies. They employed an agarose instilling technique in order to produce cylinders for slice preparation using a Krumdieck tissue slicer. They observed active biotransformation of 7-ethoxycoumarin (to 7-hydroxycoumarin plus its glucuronides and sulfates, the latter as major metabolites) and of coumarin (to metabolites of the 3-hydroxylation pathway and to minor amounts of 7-hydroxycoumarin). One year thereafter they (Price et al. 1996) used the technique to show that agaritine was biotransformed by rat (and mouse) lung slices to reactive metabolites, which covalently bound to lung proteins, less in the rat lung compared with the mouse lung.

Green et al. (1997) observed moderate metabolism of trichloroethylene in rat lung (23-fold slower than in mouse lung, but "much faster" than in human lung, where it was not detected).

Iba et al. (1998) investigated a potential induction of pulmonary EROD activity by inhalation and by subcutaneous treatment of Sprague-Dawley rats with nicotine. Pulmonary EROD activity was increased after both routes of exposure (in the female rats pulmonary EROD activity was increased 15 -fold after subcutaneous treatment and 5-fold after inhalation; in the male rat pulmonary EROD was increased 3.5-fold after subcutaneous treatment and 1.5-fold after inhalation).

Hynes et al. (1999) observed that isolated rat lung Clara (Club) cells readily metabolized styrene to styrene 7,8-oxide. Styrene metabolism was severalfold lower in rat Clara cells compared with mouse Clara cells. The less genotoxic and less cytotoxic enantiomer $(S)$-styrene 7,8-oxide was preferentially formed in rat lung microsomes, while the mouse lung microsomes formed preferentially the $(R)$-enantiomer (in mose lung Clara cells the ratio $(R)$ :(S)-epoxide was 3.98). The authors suggested that these differences are important for the lower susceptibility of the rat to styrene-induced pneumotoxicity (Hynes et al. 1999) [it should be taken into account that the $(R)$-enantiomer also is faster hydrolysed by EPHX1 (Wenker et al. 2001), such that the resulting toxicity may be smaller than expected solely from the different formation rates of the two enantiomers].

The mutagenic nitroaromatic compound 1-nitronaphthalene (emitted from diesel engines) was shown to be activated by the rat lung CYPs (Watt and Buckpitt 2000). Baldwin et al. (2005) reported 1-nitronaphthalene was bioactivated by CYP2F4 of the rat.

Lake et al. (2003) showed in precision-cut rat lung slices induction of EROD activity by BNF, aroclor 1254 and BP.

After oral dosing of propranolol to rats EROD and 7-benzyloxyresorufin O-debenzylase (BROD) activities were markedly ( $\sim 50$ to $70 \%$ ) decreased in type II pneumocyte homogenates (PROD activities remained unchanged), while BROD activities were moderately $(\sim 30 \%)$ increased 
in alveolar macrophage homogenates. The authors conclude that coadministration of oral propranolol with inhaled drugs or inhaled environmental xenobiotics could lead to unwanted interactions (Kastelova et al. 2003).

The lung-selective rat CYP2F3 (recombinant) catalyzed the biotransformation of the pneumotoxin 1,1-dichloroethylene to glutathionyl acetate putatively generated via dichloroethylene epoxide (Simmonds et al. 2004).

Umachandran et al. (2004) observed that in precision-cut rat lung slices ECOD activities were stable only for the first $8 \mathrm{~h}$. After $24 \mathrm{~h}$ only about $10 \%$ of the activity was left.

Konsue and Ioannides (2008) investigated the alleged influence of the chemopreventive anti-cancer Brassicaceae constituent phenethyl isothiocyanate on xenobiotic metabolizing enzymes. They used doses commensurate with potential human diets $(0.06 \mu \mathrm{mol} / \mathrm{g}$ diet for 14 days; which they called "low dose"), as well as higher doses (called medium and high dose, 0.6 and $6 \mu \mathrm{mol} / \mathrm{g}$, respectively). In the rat lung the microsomal CYP-dependent EROD and PROD activities were not influenced at the low and medium dose. At the high dose EROD activity was 2.7-fold increased and PROD activity was decreased to about $30 \%$ of its activity in the controls. However, Canistro et al. (2012) reported that in rat lung microsomes CYP-dependent enzymatic activities were dramatically influenced by treating rats orally with glucoraphanin-rich black cabbage seed extract (120 or $240 \mathrm{mg} / \mathrm{kg}$ body weight daily for 4 days). PROD activity was increased in male rats up to 26-fold, while EROD activity showed a loss of $86 \%$ in female rats. Some further CYP-dependent activities also were substantially modulated. The authors state that eating Brassicaceae such as cabbage after cooking, which inactivates myrosinase (the enzyme that liberates from glucosinolates their corresponding isothiocyanates), may expose people to intact glucosinolates including glucoraphanin in addition to or rather than the isothiocyanate sulforaphane, such that the claimed health effects actually may include deleterious effects due to drastic CYP activity modulations. Abdull Razis et al. (2011) found that exposure of precision-cut rat lung slices to the intact glucosinolates glucoraphanin and glucoerucin led to increases of EROD activity, which were abolished by the presence of myrosinase.

Yilmaz et al. (2018) observed that the metabolite M3 of the anti-Parkinson drug mavoglurant is produced mainly by CYP1A1. Accordingly, only traces of M3 were found in hepatic systems (known to constitutively possess only very low/negligible amounts of CYP1A1 protein), but significant amounts of this metabolite were produced by rat lung microsomes and tissue slices (albeit relatively low amounts, which suggests that other extrahepatic organs also contribute to this reaction).

Yilmaz et al. (2019) observed in rat precision-cut lung slices low, but clearly quantifiable CYP3A4 activity toward midazolam (about $0.3 \%$ substrate conversion in $4 \mathrm{~h}$ ) and toward the CYP2C substrates amodiaquine (about $0.6 \%$ conversion), diclofenac (about $0.4 \%$ conversion), and diazepam (about $2 \%$ conversion) (while in human precision-cut lung slices no conversion was detected). The CYP1A1 substrate AFQ056 showed about 30\% conversion by the rat lung slices, and extremely little conversion (less than $0.1 \%$ estimated from the figure in Yilmaz et al. 2019) by the human lung slices.

\section{Non-CYP oxidoreductases}

\section{Flavin-dependent monoxygenase (FMO)}

FMOs occur in the mammalian liver and appear to be important xenobiotica-metabolizing oxidoreductases in extrahepatic tissues, in the latter possibly more important than CYPs. However, their substrate specificity is limited to soft nucleophilic centers most notably converting nitrogen and sulfur atoms in xenobiotic molecules to $\mathrm{N}$-oxides an $S$-oxides.

Sukumaran et al. (2011) reported the presence of FMO2 and FMO3 mRNA in the rat lung and circadian oscillations of their levels.

Yilmaz et al. (2018) observed in rat precision-cut lung slices substantial FMO activity toward benzydamine (about $80 \%$ substrate conversion in $4 \mathrm{~h}$ ) (while in human precisioncut lung slices only about $0.7 \%$ of the substrate was converted by FMO). These FMO activities were much higher than the CYP activities in rat (and human) lung slices observed in the same study (Yilmaz et al. 2019).

\section{NAD(P)H:quinone oxidoreductase (NQO)}

NQOs protect against quinone toxicity by their direct 2-electron reduction avoiding semiquinone radicals as primary metabolites. NQOs convert the carbonyl function to the hydroxyl function which usually serves as substrate for conjugation by glucuronsyltransferases and/or sulfotransferases followed by facilitated excretion. NADH or NADPH may serve as reducing equivalent.

Gate et al. (2006) reported the presence of and a considerable increase (6.7-fold) in the NQO1 mRNA level in the rat lung upon exposure of the animals to bitumen fumes.

Hanlon et al. (2009) showed in precision-cut rat lung slices upon treatment of the slices with the aliphatic isothiocyanates sulforaphane or erucin marked increases in NQO protein and activity, the latter determined with 3-(4,5-dimethylthiazo2-yl)-2,5-diphenyltetrazolium bromide as substrate. Sulforaphane led to a modest increase in CYP3A2 mRNA as well. Abdull Razis et al. (2011) found that exposure of precisioncut rat lung slices to the intact glucosinolates glucoraphanin and glucoerucin did not lead to increases of NQO activity, but 
addition of myrosinase, which liberates the isothiocyanates from the glucosinolates, led to increases of NQO activity.

\section{Alcohol dehydrogenase (ADH)}

ADHs convert alcohols to aldehydes or ketones. Ethanol is converted by ADH to acetaldehyde which is chiefly responsible for ethanol's acute toxicity. NAD serves as electron acceptor.

Bernstein (1982) showed in rat lung slices remarkable ethanol metabolizing activity $\left(V_{\max } 450 \mathrm{nmol} / \mathrm{g}\right.$ lung/h). At $10 \mathrm{mM}$ ethanol rat lung slices metabolized ethanol ten times faster than rat liver slices, leading to the calculation that rat lung metabolism could account for $30-40 \%$ of total in vivo ethanol metabolism.

\section{Aldehyde dehydrogenase (ALDH)}

ALDHs convert aldehydes to the corresponding carboxylic acids. This step usually represents a detoxication, but in the important case of methanol the conversion of its metabolite formaldehyde by ALDH leads to formic aid which is chiefly responsible for methanol's acute toxicity. Depending on the ALDH isoenzyme in question, NAD or NADP may serve as electron acceptor.

Yoon et al. (2006) studied the postnatal development of ALDH in the rat lung (and liver). ALDH1 protein levels were at their maximum at postnatal day (PND) 1 and decreased thereafter (both, in lung and liver). ALDH2 and ALDH3 drastically increased in the lung from PND1 to PND60. The only detectable ALDH3 in the lung was ALDH3A1. Lung ALDH was mostly cytosolic (in contrast to the liver where ALDH was mostly mitochondrial).

Gate et al. (2006) reported a 50-fold increase in the ALDH3A1 mRNA level in the rat lung upon exposure of the animals to bitumen fumes.

\section{Aldehyde oxidase (AO)}

AO catalyzes the conversion of aldehydes to carboxylic acids by simultaneous formation of hydrogen peroxide. It uses molecular oxygen as electron acceptor.

Yilmaz et al. (2019) observed in rat precision-cut lung slices AO activity toward carbazeran (about $0.9 \%$ substrate conversion in $4 \mathrm{~h}$ ) (while in human precision-cut lung slices about $0.2 \%$ of carbazeran was converted by $\mathrm{AO}$ ).

\section{Hydrolases}

\section{Epoxide hydrolase (EH)}

EHs convert electrophilically reactive epoxides to much less reactive diols by addition of water to the epoxide function.
This usually represents a detoxication except where the diols formed are precursors of even more toxic ultimate metabolites as in the case of pre-bay-region diols derived from angular PAHs.

Microsomal epoxide hydrolase $(\mathrm{mEH}$, also called EPHX1) Expression on the protein level Antibody raised against rat liver $\mathrm{mEH}$ stained rat lung bronchial epithelia, Clara (Club) as well as ciliated bronchiolar cells and alveolar wall cells, quantitatively strongest Clara cells (about 2-fold compared with the other cells) (Baron and Voigt 1990). Thus, the location of $\mathrm{mEH}$ in rat lung is similar to that of CYP reductase and CYP2B2 and dissimilar to that of CYP $1 \mathrm{~A} 1$ and $3 \mathrm{~A} 2$ discussed above. The relative distribution of $\mathrm{mEH}$ remained unchanged after treatment of the rats with 3-methylcholanthrene or Aroclor 1254 (Baron and Voigt 1990). De Waziers et al. (1990) determined the specific (i.e. per mg protein) amount of $\mathrm{mEH}$ protein in rat lung as $6 \%$ compared with the specific amount found in the rat liver. Pushparajah et al. (2007) reported induction of $\mathrm{mEH}$ protein in precision-cut rat lung slices by BP and activity toward BP 4,5-oxide. In the subsequent year (Pushparajah et al. 2008a), they reported induction of $\mathrm{mEH}$ protein by $\mathrm{BP}$, dibenzo[a,1] pyrene and 1-methylphenanththrene in precision-cut rat lung slices, comparatively much more pronounced than in rat liver slices (Table 4).

mEH activity (Table 5) Early on Oesch et al. (1977) investigated $\mathrm{mEH}$ activities in 25 extrahepatic organs of the rat. Specific activities were remarkably high in the lung, fourth after liver, testis and kidney. Schmassmann et al. (1978) and Lorenz et al. (1979) also observed considerable activities of microsomal epoxide hydrolase in rat lung. In the former study they observed lack of rat lung $\mathrm{mEH}$ inducibility by several epoxides, including long-lived, stable epoxides. VogelBindel et al. (1982) demonstrated relatively high activities of rat lung $\mathrm{mEH}$ toward the steroid epoxides estroxide and androstene oxide, the ratios of activities compared with the rat hepatic $\mathrm{mEH}$ being similar as for $\mathrm{BP} 4,5$-oxide.

Jones et al. (1983) reported much higher $\mathrm{mEH}$ activity in rat lung Clara cells as compared with type II pneumocytes.

DePierre et al. (1984) observed EH activities in rat lung $10,000 \mathrm{~g}$ supernatants (hence the sum of $\mathrm{mEH}$ and $\mathrm{sEH}$ ) which were not increased by the standard inducers of many xenobiotic metabolizing enzymes phenobarbital, 3-methylcholanthrene or trans-stilbene oxide.

Boogaard and Bond (1996) reported that rat lung microsomes hydrolyzed diepoxybutane with a much higher $V_{\max } / K_{\mathrm{m}}$ efficiency (19.2) than the mouse (3.93) providing a likely contribution for the much lower susceptibility to the carcinogenicity of the parent compound butadiene of the rat lung compared with the mouse lung.

Sukhanov et al. (2009) reported on rats, which were kept in vivariums, marked seasonal changes in pulmonary $\mathrm{mEH}$ 
Table 4 Non-CYP-mediated oxidoreductase activities in lung of various mammalian species

\begin{tabular}{|c|c|c|c|c|c|}
\hline Substrate (for) & Human & Rat & Mouse & Rabbit & References \\
\hline Propanal $\left(\mathrm{ALDH}^{\mathrm{a}}\right)$ & - & $1.5 \pm 0.1^{\mathrm{b}}$ & - & - & Yoon et al. (2006) \\
\hline Hexanal (ALDH) & - & $0.4 \pm 0.1^{\mathrm{b}}$ & - & - & Yoon et al. (2006) \\
\hline Benzaldehyde (ALDH) & - & $1.5 \pm 0.2^{\mathrm{b}}$ & - & - & Yoon et al. (2006) \\
\hline $\begin{array}{l}\text { 2,6-Dichlorophenolindophe- } \\
\text { nol }\left(\mathrm{NQO}^{\mathrm{a}}\right)\end{array}$ & - & - & $\sim 52^{\mathrm{d}}$ & - & $\begin{array}{l}\text { Kalpana Deepa } \\
\text { Priya et al. } \\
\text { (2011) }\end{array}$ \\
\hline Naloxone $\left(\mathrm{AKR}^{\mathrm{a}}\right)$ & - & - & - & $0.56^{\mathrm{c}}$ & Endo et al. (2014) \\
\hline Loxoprofen $\left(\mathrm{AKR}^{\mathrm{a}}\right)$ & - & - & - & $0.62^{\mathrm{c}}$ & Endo et al. (2014) \\
\hline Metyrapone $\left(\mathrm{AKR}^{\mathrm{a}}\right)$ & - & - & - & $0.95^{\mathrm{c}}$ & Endo et al. (2014) \\
\hline
\end{tabular}

Only constitutive activities; unless otherwise stated values either are mean \pm SD or there was no indication in the original literature; -, no comparable values found

a Abbreviations for enzymes: $A D H$ alcohol dehydrogenase, $A K R$ Aldo-keto reductase, $A L D H$ aldehyde dehydrogenase, $N Q O \mathrm{NAD}(\mathrm{P}) \mathrm{H}$ quinone oxidoreductase

${ }^{\mathrm{b}} \mathrm{nmol}$ product/mg cytosolic protein/min

${ }^{\mathrm{c}} k_{\text {cat }}$ per min

${ }^{\mathrm{d}} \mathrm{nmol}$ 2,6-dichlorophenolindophenol reduced/min/mg cytosolic protein

Table 5 Xenobiotic hydrolase activities in lung of various mammalian species

\begin{tabular}{|c|c|c|c|c|c|}
\hline Substrate (for) & Human & Rat & Mouse & Dog & References \\
\hline Para-nitrophenyl acetate $\left(\mathrm{E}^{\mathrm{a}}\right)$ & $19.0-48.2$ & & & & Forkert et al. (2001) \\
\hline Beclomethasone dipropionate (E) & $33 \pm 23^{\mathrm{d}}$ & & & & Somers et al. (2007) \\
\hline Phenanthrene 9,10-oxide $\left(\mathrm{mEH}^{\mathrm{a}}\right)$ & $3590 \pm 250^{c}$ & & & & Lorenz et al. (1979) \\
\hline Benz[a]anthracene 5,6-oxide (mEH) & $920 \pm 120^{\mathrm{b}}$ & & & & Lorenz et al. (1979) \\
\hline \multirow[t]{3}{*}{ Benzo[a]pyrene 4,5-oxide (mEH) } & $1219 \pm 428^{\mathrm{b}, \mathrm{f}}$ & $362 \pm 46^{\mathrm{b}} ; 384 \pm 21^{\mathrm{b}}$ & $427 \pm 148^{\mathrm{b}}$ & & Oesch et al. $(1977,1980)$ \\
\hline & $773 \pm 114^{\mathrm{b}} ; 739 \pm 189^{\mathrm{b}, \mathrm{e}}$ & $239 \pm 33^{b, j}$ & & & $\begin{array}{l}\text { Lorenz et al. (1979), Oesch } \\
\text { et al. (1980), Umachan- } \\
\text { dran and Ioannides (2006) }\end{array}$ \\
\hline & & $154.2 \pm 19.1^{\mathrm{k}}$ & & & Jones et al. (1983) \\
\hline 7-Methylbenz[a]anthracene 5,6-oxide (mEH) & $1280 \pm 90^{\mathrm{b}}$ & & & & Lorenz et al. (1979) \\
\hline 3-Methylcholanthrene 11,12 -oxide $(\mathrm{mEH})$ & $390 \pm 60^{b}$ & & & & Lorenz et al. (1979) \\
\hline \multirow[t]{2}{*}{ Styrene 7,8-oxide $(\mathrm{mEH})$} & $0.43 \pm 0.07^{\mathrm{h}} ; 1.08 \pm 0.14^{\mathrm{h}}$ & & $8.02 \pm 0.77^{\mathrm{h}}$ & $\sim 10^{\mathrm{g}}$ & $\begin{array}{l}\text { Schmassmann et al. (1978), } \\
\text { Pacifici et al. (1988b), } \\
\text { Carlson (1998), Bond } \\
\text { et al. (1988) }\end{array}$ \\
\hline & & $1.8-2.5^{\mathrm{h}}$ & $2.1^{\mathrm{h}}$ & & Mendrala et al. (1993) \\
\hline Cis-stilbene oxide (mEH) & $\sim 0.5^{\mathrm{g}, \mathrm{i}}$ & & & & Fanucchi et al. (2000) \\
\hline Trans-stilbene oxide $\left(\mathrm{sEH}^{\mathrm{a}}\right)$ & $8.5 \pm 2.8^{\mathrm{c}}$ & & & & Pacifici et al. (1988a) \\
\hline Diepoxybutane (mEH) & $21.7 \pm 1.9^{\mathrm{h}}$ & $19.3 \pm 7.8^{\mathrm{h}}$ & $49.8 \pm 9.7^{\mathrm{h}}$ & & Boogaard and Bond (1996) \\
\hline
\end{tabular}

Only constitutive activities; unless otherwise stated values either are mean \pm SD or there was no indication in the original literature

${ }^{a}$ Abbreviations: $E$ esterase, $m E H$ microsomal epoxide hydrolase (EPHX1), $s E H$ soluble epoxide hydrolase (EPHX2)

${ }^{\mathrm{b}} \mathrm{pmol} / \mathrm{min} / \mathrm{mg}$ microsomal protein

${ }^{\mathrm{c}} \mathrm{pmol} / \mathrm{min} / \mathrm{mg}$ 105,000 g supernatant fraction

${ }^{\mathrm{d}}$ Clearance $\mathrm{ml} / \mathrm{min} / 10^{6}$ cells in lung parenchymal cells

${ }^{\mathrm{e}}$ Nonsmokers

${ }^{\mathrm{f}}$ Smokers

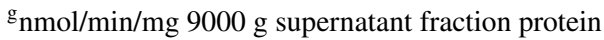

${ }^{\mathrm{h}} \mathrm{nmol} / \mathrm{min} / \mathrm{mg}$ microsomal protein

${ }^{\mathrm{i}}$ Distal airways

${ }^{\mathrm{j}}$ Precision-cut lung slices

${ }^{\mathrm{k}} \mathrm{pmol} / \mathrm{min} / 10^{6} 50 \%$ Clara cells 
activities (for phenanthrene 9,10 oxide as substrate), with two peaks, a steep peak in March (3- to 4-fold difference compared with the preceding lowest values) and a smoother peak in July to August. In contrast to the liver, the $\mathrm{mEH}$ activity in the lung was higher in females than in males.

Soluble epoxide hydrolase (sEH, also called EPHX2) sEH levels are relatively low in the (adult male Sprague-Dawley) rat lung compared with other organs (heart $>$ kidney $>$ liver $>$ brain $>$ lung $>$ testis $>$ spleen). The specific activity in the rat lung was $15 \%$ of that observed in the rat liver (Oesch et al. 1986; Schladt et al. 1986).

The team of Bruce Hammock (Smith et al. 2005) showed that $\mathrm{SEH}$ is involved in tobacco-smoke induced lung inflammation, which can be substantially attenuated by selectively inhibiting sEH activity.

\section{Esterases}

Esterases cleave esters (and in many cases also amides) to the corresponding alcohols (or amines, respectively) and acids. The selective presence of individual esterase isoenzymes and their substrate/inhibitor specificity are the basis for the selective toxicity of organophosphates to insects while mammals are largely protected.

Sukumaran et al. (2011) reported the presence of CES3 (carboxylesterase 3) mRNA in the rat lung and circadian oscillations of its levels.

Yilmaz et al. (2019) observed in rat precision-cut lung slices esterase activity toward the CES 1 substrate ramipril (between 10 and 20\% substrate conversion in $4 \mathrm{~h}$ ) (while in human precision-cut lung slices about $7 \%$ of the substrate were hydrolyzed).

\section{Conjugating enzymes}

\section{Glutathione S-transferase (GST)}

GSTs convert electrophilically reactive xenobiotics to their glutathione (GSH) conjugates. This is usually a detoxication, but in some cases a toxication (e.g. by generating threemembered sulfonium containing heterocycles).

GST transcripts Mainwaring et al. (1996) demonstrated the presence of GSTT transcripts in the rat lung, especially in Clara (Club) cells. Levels were markedly lower than in the mouse lung.

Gate et al. (2006) reported a significant increase (2-fold) in the GSTA5 mRNA level in the rat lung upon exposure of the animals to bitumen fumes.

Sukumaran et al. (2011) reported the presence of GSTA3 mRNA in the rat lung and circadian oscillations of its levels.
Samuelsen et al. (2012) reported that exposure of rat lung primary alveolar type 2 cells to 2 -hydroxyethyl-methacrylate (HEMA) led to nuclear translocation of the AhR and to induction of GSTA2 (and CYP1A1 and CYP1B1) mRNA (known to be AhR-dependent), but not to an increase in CYP2B1 or CYP2E1 mRNA (known not to be AhR-dependent).

GST protein GST 1-2 and GST 3-4 protein and a GST form, which the authors assumed to be unique to the lung, were observed throughout the rat lung alveolar wall and bronchiolar epithelium (Awasthi et al. 1984). Baron and Voigt (1990) localized the GST proteins GST 1-1, 3-4, and 5-5 in the untreated rat lung to the bronchial epithelia, Clara (Club) and ciliated bronchiolar cells as well as alveolar wall cells. The levels of all of them were lowest in type II pneumocytes, GST 1-1 levels highest in Clara cells, GST 3-4 levels highest in bronchial epithelia, GST 5-5 levels similar in Clara cells and bronchial epithelia (Baron and Voigt 1990). The relative distribution of the investigated GSTs remained unchanged after treatment of the rats with 3-methylcholanthrene or Aroclor 1254 (Baron and Voigt 1990). Mainwaring et al. (1996) demonstrated the presence of GST theta protein in the rat lung, especially in Clara cells. Levels were markedly lower in the rat lung compared with the mouse lung providing a possible explanation for the species specificity of the mouse carcinogen methylene chloride, which is metabolically activated by GST.

Hanlon et al. (2009) showed in precision-cut rat lung slices modest increases in GSTalpha protein upon treatment of the slices with the aliphatic isothiocyanates sulforaphane or erucin. GSTpi protein levels remained unchanged. Abdull Razis et al. (2011) observed increases in GSTalpha protein levels also upon exposure of the precision-cut rat lung slices to the isothiocyanate precursor glucosinolates, glucoraphanin and glucoerucin.

GST catalytic activities (Table 6) Very early on Lorenz et al. (1979) already observed considerable activities of GST for BP 4,5-oxide in rat lung cytosol (Table 6).

Autrup et al. (1980) showed in cultured rat tracheobronchial tissue a high proportion of BP metabolized to glutathione metabolites, much more than to glucuronides. Jones et al. (1983) reported that benzo[a]pyrene 4,5-oxide was much more extensively conjugated with GSH in rat Clara cells compared with type II pneumocytes (unknown whether due to GST activity or GSH levels or both).

Oesch et al. (1983) reported for the rat lung cytosol activities for several substrates prototypic for different GSTs (see Table 6). All of them were modestly, but significantly inducible by Aroclor 1254. All activities were lower in the lung compared with the liver, most of them 10- to 50-fold lower. 
Table 6 Glutathione $S$-transferase (GST) activities in lung of various mammalian species

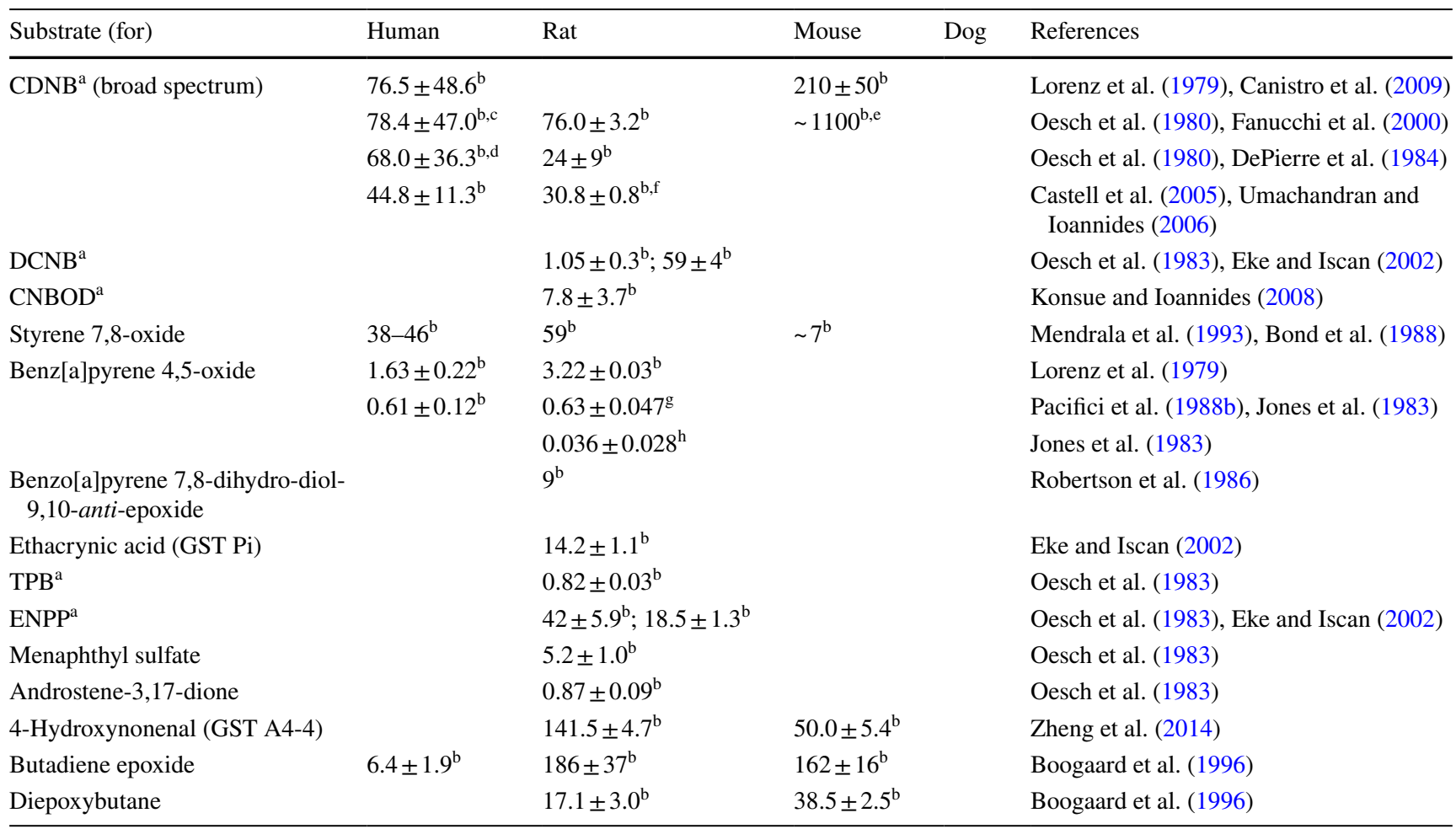

Only constitutive activities; unless otherwise stated values either are mean \pm SD or there was no indication in the original literature

${ }^{a}$ CDNB, 1-chloro-2,4-dinitrobenzene; CNBOD, 7-chloro-4-nitrobenzo-2-oxa-1,3-diazole; cytos, cytosol; DCNB, 1,2-dichloro-4-nitrobenzene; ENPP, 1,2-epoxy-3-( $p$-nitrophenoxy)-propane; GST, glutathione $S$-transferase; TPB, trans-4-phenyl-3-butene-2-one

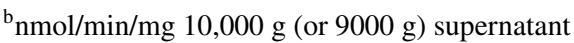

${ }^{\mathrm{c}}$ Nonsmokers

${ }^{\mathrm{d}}$ Smokers

${ }^{\mathrm{e}}$ Distal airways

${ }^{\mathrm{f}}$ Precision-cut lung slices

${ }^{\mathrm{g}} \mathrm{nmol} / \mathrm{min} / 10^{6} 30 \%$ Clara cells

${ }^{\mathrm{I}} \mathrm{nmol} / \mathrm{min} / 10^{6} 80$ type II pneumocytes

DePierre et al. (1984) observed GST activities in rat lung $10,000 \mathrm{~g}$ supernatants, which were not increased by the standard inducers of many xenobiotic metabolizing enzymes phenobarbital, 3-methylcholanthrene or trans-stilbene oxde. Similarly, Pushparajah et al. (2008b) observed in precision-cut rat lung slices no increase in GST activity toward the broad-spectrum substrate CDNB (1-chloro-2,4-dinitrobenzene) upon treatment with the polycyclic aromatic hydrocarbon (PAH) benzo[b]fluoranthene (whereas in rat liver slices marked increases were noted).

Robertson et al. (1986) observed highly efficient activity of the rat lung GST 7-7 toward the ultimately carcinogenic BP metabolite (+)-7 $\beta, 8 \alpha$-dihydroxy- $9 \alpha, 10 \alpha$-oxy-7,8,9,10tetrahydro-BP. Rat lung GST 4-4 was the second most active isozyme, the catalytic efficiency $(\mathrm{kcat} / \mathrm{km})$ being 7 -fold lower.
Boogaard et al. (1996) observed considerable GST activity toward diepoxybutane $(17.1 \pm 3.0 \mathrm{nmol} / \mathrm{min} / \mathrm{mg}$ cytosolic protein) in rat lung cytosol, about half of the activity observed in the mouse lung $(38.5 \pm 2.5 \mathrm{nmol} / \mathrm{min} / \mathrm{mg}$ cytosolic protein). They concluded that differences between GST activities are not the reason for the fact that rat upon exposure to butadiene accumulates lower concentrations of diepoxybutane in the blood than the mice, which are much more susceptible to the carcinogenicity of butadiene.

Eke and Iscan (2002) reported for rat lung 10,000 g supernatant GST activity toward CDNB, 1,2-dichloro4-nitrobenzene, ethacrynic acid and 1,2-epoxy-3-( $p$ nitrophenoxy)-propane. All of these activities increased after exposure of the rats to smoke from high-tar cigarettes ( $32 \mathrm{mg} /$ cigarette), while only the activity toward 1,2-dichloro-4-nitrobenzene was increased after exposure to smoke from low-tar cigarettes $(15 \mathrm{mg} /$ cigarette $)$. In the 
study by Huber et al. (2002) treatment of rats with the coffee constituents kahweol and cafestol (mixed in the diets) led to a modest, but significant increase by $37 \%$ in the rat lung 10,000 g supernatant of GST activity toward CDNB. This was interpreted as a potential contribution to the chemoprevention of DNA adduct formation by these coffee constituents, which had been shown in an earlier study (Huber et al. 1997). Hanlon et al. (2009) showed in precision-cut rat lung slices marked increases in GST activity determined with CDNB as substrate upon treatment of the slices with the aliphatic isothiocyanates sulforaphane or erucin (sulforaphane led to a modest increase in CYP3A2 mRNA as well). Abdull Razis et al. (2011) observed increases in GST activity toward CDNB as substrate also upon exposure of the precision-cut rat lung slices to the isothiocyanate precursor glucosinolate, glucoraphanin.

Li et al. (2011a) observed in rat pulmonary cytosol GST activity toward ethylene oxide as substrate. Zheng et al. (2014) observed that GST activity toward the prototypic substrate 4-hydroxynonenal was very high in rat lung $9000 \mathrm{~g}$ supernatant, higher than in the liver.

\section{UDP-glucuronosyltransferase (UGT) (Table 7)}

UGTs convert xenobiotics which possess appropriate substituents (mostly hydroxyl, sometimes amino, sulfhydryl or carboxyl) to glucuronides. This represents usually a detoxication, but in some cases (e.g. "acyl migration") a toxication.

UGT protein levels Using microfluorimetric determination of antibody binding, Baron and Voigt (1990) reported the finding that $p$-nitrophenol UGT and also $3 \alpha$-hydroxysteroid UGT proteins (or closely related antigens) occurred at similar levels in untreated rat bronchial epithelia, Clara (Club) cells and type II pneumocytes. $3 \alpha$-Hydroxysteroid UGT antibody bound at markedly lower levels in all three cell types. 17- $\beta$-Hydroxysteroid UGT antibody bound markedly less in type II pneumocytes compared with the other two

Table 7 UDP-glucuronosyl (UGT), sulfotransferase (SULT) and $N$-acetyltransferase (NAT) activities in lung of various mammalian species

\begin{tabular}{|c|c|c|c|c|c|}
\hline Substrate (for) & Human & Rat & Mouse & Dog & References \\
\hline Umbelliferone & & & & $\sim 0.15^{\mathrm{g}}$ & Bond et al. (1988) \\
\hline \multirow[t]{2}{*}{ 4-Methylumbelliferone (UGT ${ }^{\mathrm{a}}$ ) } & $\mathrm{bd}^{\mathrm{h}}$ & $189.1 \pm 54.0^{\mathrm{d}}$ & & & Somers et al. (2007), Jones et al. (1983) \\
\hline & $0.048 \pm 0.071^{\mathrm{b}}$ & $32.4 \pm 9.7^{\mathrm{e}}$ & & & Castell et al. (2005), Jones et al. (1983) \\
\hline 4-Nitrophenol & & & $\sim 10^{\mathrm{b}}$ & & Kalpana Deepa Priya et al. (2011) \\
\hline Bilirubin (UGT) & & $\sim 0.023^{\mathrm{b}}$ & & & Yokota et al. (2002) \\
\hline 7-Hydroxycoumarin (UGT) & $b d^{h}$ & $\sim 10^{\mathrm{i}}$ & & & Somers et al. (2007), De Kanter et al. (2002b) \\
\hline \multirow[t]{3}{*}{ 1-Naphthol (UGT) } & Trace $^{1}$ & & $0.12 \pm 0.001^{\mathrm{b}}$ & & Somers et al. (2007), Canistro et al. (2009) \\
\hline & $0.01 \pm 0.001^{\mathrm{b}}$ & $\sim 0.27^{\mathrm{b}}$ & & & Pacifici et al. (1988b), Yokota et al. (2002) \\
\hline & & $0.63 \pm 0.08^{\mathrm{b}, \mathrm{c}}$ & & & Umachandran and Ioannides (2006) \\
\hline Acetaminophen (UGT) & $b d^{h}$ & & & & Somers et al. (2007) \\
\hline Acetaminophen $\left(\right.$ SULT $\left.^{\mathrm{a}}\right)$ & $\mathrm{bd}^{\mathrm{h}}$ & & & & Somers et al. (2007) \\
\hline 7-Hydroxycoumarin (SULT) & $\mathrm{bd}^{\mathrm{h}} ; \sim 15^{\mathrm{i}}$ & & & & Somers et al. (2007), De Kanter et al. (2002b) \\
\hline 1-Naphthol (SULT) & $2.11 \pm 2.03^{\mathrm{h}}$ & & & & Somers et al. (2007) \\
\hline 2-Naphthol (SULT) & $66.5 \pm 15.7^{f}$ & $3540 \pm 1850^{\mathrm{f}, \mathrm{c}}$ & & & $\begin{array}{l}\text { Pacifici et al. (1988b); Umachandran and Ioannides } \\
\text { (2006) }\end{array}$ \\
\hline Naloxone (SULT) & $38.1 \pm 0.3^{\mathrm{f}}$ & & & & Kurogi et al. (2012) \\
\hline Buprenorphin (SULT) & $<\sim 0.1^{\mathrm{f}}$ & & & & Kurogi et al. (2012) \\
\hline Pentazocine (SULT) & $<\sim 0.1^{\mathrm{f}}$ & & & & Kurogi et al. (2012) \\
\hline Para-aminobenzoic acid (NAT ${ }^{\mathrm{a}}$ ) & $1.19 \pm 0.15^{\mathrm{g}}$ & & & & Pacifici et al. (1986) \\
\hline
\end{tabular}

Only constitutive activities; unless otherwise stated values either are mean \pm SD or there was no indication in the original literature

a Abbreviations: NAT N-acetyltransferase, SULT sulfotransferase, UGT UDP-glucuronosyltransferase

${ }^{\mathrm{b}} \mathrm{nmol}$ product/min/mg microsomal protein

${ }^{\mathrm{c}}$ Precision-cut lung slices

${ }^{\mathrm{d}} \mathrm{pmol} / \mathrm{min} / 10^{6} 50 \%$ Clara cells

${ }^{\mathrm{e}} \mathrm{pmol} / \mathrm{min} / 10^{6} 80 \%$ type II pneumocytes

${ }^{\mathrm{f}} \mathrm{pmol} / \mathrm{min} / \mathrm{mg} 9000 \mathrm{~g}$ supernatant protein

${ }^{\mathrm{g}} \mathrm{nmol} / \mathrm{min} / \mathrm{mg} 9000 \mathrm{~g}$ supernatant protein

${ }^{\mathrm{h}}$ Clearance $\mathrm{ml} / \mathrm{min} / 10^{6}$ cells in lung parenchymal cells

${ }^{\mathrm{i}}$ nmol product $/ 3 \mathrm{~h} / \mathrm{mg}$ slice 
cell types. The relative distribution of the investigated UGTs remained unchanged after treatment of the rats with 3-methylcholanthrene or Aroclor 1254.

UGT activity Bock et al. (1980) reported that rat lung possesses UGT activity toward 1-naphthol and that this activity is increased by pretreatment of the rats with Aroclor 1254 .

Jones et al. (1983) observed 4-methylumbelliferone glucuronidation in rat lung Clara cells and considerably lower activity in type II pneumocytes. In the latter cell type the activity was increased by $\beta$-naphthoflavone.

Yokota et al. (2002) observed in the rat lung very low activities of UGT toward 1-naphthol, which were further decreased to $20-30 \%$ of controls in hypophysectomized rats. Activities toward bilirubin in the rat lung were extremely low, but remained unchanged in hypophysectomized rats.

Yilmaz et al. (2019) observed in rat precision-cut lung slices quite remarkable UGT activity toward triclosan (about $40 \%$ substrate conversion in $4 \mathrm{~h}$ ) as opposed to very low activity in human precision-cut lung slices (about $0.1 \%$ conversion). About 5\% 4-methylumbelliferone were glucuronidated by the rat lung slices as compared to somewhat less than $0.2 \%$ by the human lung slices.

\section{Sulfotransferase (SULT)}

SULTs convert xenobiotics which possess appropriate substituents (mostly hydroxyl, sometimes amino, sulfhydryl or carboxyl) to sulfates. This conversion usually represents a detoxication, but in some cases it rather represents a toxication (e.g. converting benzylic alcohols to reactive esters).

Baron and Voigt (1990) reported the binding of antibody raised against rat liver aryl sulfotransferase IV to untreated rat lung bronchial epithelia, bronchiolar Clara (Club) and ciliated cells as well as type II pneumocytes and other alveolar wall cells, 15-25\% more to bronchial epithelia compared with the other cell types. They investigated the relative distribution of aryl sulfotransferase IV also after treatment of the rats with 3-methylcholanthrene or Aroclor 1254 and found it to remain unchanged.

Autrup et al. (1980) showed in cultured rat tracheobronchial tissue a high proportion of BP metabolized to sulfates, much more than to glucuronides.

Yilmaz et al. (2019) observed in rat precision-cut lung slices SULT activity toward triclosan (about 3\% substrate conversion in $4 \mathrm{~h}$ ) (while in human precision-cut lung slices about $40 \%$ of the substrate was converted by SULT). About 20\% 4-methylumbelliferone were converted by the rat lung slices SULT (as compared to about $7 \%$ by the human lung slices).

Sukumaran et al. (2011) reported circadian oscillations of SULT1A1 mRNA levels in the rat lung.

\section{$N$-Acetyltransferases (NAT)}

Depending on the relative speed of the individual reactions NATs detoxify or toxify: if in aromatic amines the oxidative formation of the hydroxylamine is much faster than the transfer of the acetyl group to the original amine, the acetyl moiety may be transferred to the oxygen atom generating acetoxy as a good leaving group (leaving as acetate) which leaves behind an electrophilically reactive ammonium ion.

Walraven et al. (2007) and Barker et al. (2008) showed that NAT1 and NAT2 transcripts were present in rat lung at roughly similar levels as in the liver, NAT2 transcripts even somewhat more abundant, while in all 14 tissues investigated (including the lung) their newly discovered NAT3 was expressed at very low levels ( 3 orders of magnitude less than NAT1 and NAT2).

Yilmaz et al. (2019) observed in rat precision-cut lung slices NAT activity toward p-toluidine (about $50 \%$ substrate conversion in $4 \mathrm{~h}$ ) (while in human precision-cut lung slices about $30 \%$ of the substrate were acetylated).

\section{Xenobiotica-metabolizing enzymes in the mouse lung}

\section{Cytochromes P450 (CYP)}

\section{CYP transcript expression}

Of 14 mouse organs investigated CYP1A1, CYP2B 10 and CYP4B1 constitutive mRNA levels were highest in the lung. Levels of CYP2F2 and CYP2S1 mRNA also were very high in the mouse lung, but-in contrast to earlier studies which did not include stomach and which consequently found highest levels of CP2S1 mRNA in the lung (Choudhary et al. 2003) - the lung was now with respect to CYP2S1 mRNA levels outperformed by the stomach (Renaud et al. 2011).

Jin et al. (2004) investigated possible reasons for the observation that-in contrast to CYP1A1 and 1B1 mRNACYP1A2 transcripts are not found in the mouse lung, but are abundant in the mouse liver. They found that in these two organs in the promoter's 5'-flanking region the $\mathrm{CpG}$ methylation pattern behaved differently in three regions, which they called R1, R2 and R3. In the $\mathrm{CpG}$ richest region, $\mathrm{R} 3$, the CpGs were almost completely methylated in both tissues, lung and liver. However, in R1 90\% were methylated in the lung compared with $6.7 \%$ in the liver and in R2 72.2\% were methylated in the lung compared with $5.6 \%$ in the liver, providing a possible explanation for CYP1A2 transcripts not being found in the lung.

Imaoka et al. (1995) observed in the mouse lung (relatively low levels of) CYP4B1 mRNA (but they could not detect CYP4B1 protein), while Choudhary et al. (2005) 
reported high levels of CYP4B1 transcripts in the mouse lung.

Rivera et al. (2002) reported the finding of the transcript for a novel mouse CYP, CYP2S1, which was induced in mouse lung (and liver) by TCDD [requiring the presence of AhR (aryl hydrocarbon receptor) and ARNT (AhR translocator)], the first observation of an induction by TCDD of a CYP outside the CYP1 family.

Choudhary et al. (2003) reported the constitutive expression of 24 Cyp genes in mouse embryonic or adult lung. In the adult mouse CYP2S $1 \mathrm{mRNA}$ was found in all four extrahepatic tissues investigated, including lung. CYP2F2, 2S1 and 4B1 mRNA were major transcripts in the lung. CYP2G1 mRNA was specific for the lung. CYP3A25 was found only in the liver and lung. During embryonic development CYP2F2 mRNA appeared at embryonic day 11 , increasing to relatively high levels at embryonic days 15-17.

Baldwin et al. (2004) observed that mRNA coding for CYP2F, which is converting the selective mouse Clara (Club) cell pneumotoxin naphthalene to its reactive metabolite naphthalene 1,2-epoxide, is highly expressed in mouse lung, much more than in the mouse liver, while in the mouse kidney no CYP2F transcripts were found. In the Rhesus Macaque airways no mRNA coding for CYP2F was found.

Choudhary et al. (2005) reported the expression of stageselective expression of CYP genes in the mouse embryo: Cyp 1al and $2 r 1$ were expressed only in stage E7, Cyp2e1 only in stage E17, while CYP2S1 mRNA was present in all stages. The authors suggest that the stage-selective expressions may be involved in development.

Theken et al. (2011) investigated the influence of the acute inflammatory response on eicosanoid metabolism and potentially related CYP expression. They observed the following CYP transcripts in the mouse lung: CYP2C 44 , 2J5, 2J9, 4A12a, 4A12b, 4F13 and 4F6. CYP2J9 was the most abundant. On the other end, CYP2C29 mRNA was not detected. The activator of innate immune response LPS (lipopolysaccharide) decreased the level of CYP2J9, 2C44, 4A12a, 4A12b, 4F13 and 4F16.

Tanimura et al. (2011) reported that Cyplal gene expression in the mouse lung was under CLOCK-dependent circadian rhythm control.

Theken et al. (2011) reported high mRNA levels of CYP2J9 and 4F13, moderate levels of CYP4A12a, 4A12b and $4 \mathrm{~F} 16$, very low levels of CYP2C44 and $2 \mathrm{~J} 5$ in the mouse lung, while they did not detect CYP2C29 mRNA. Treatment of the mice with lipopolysaccharide led to a decrease of all of them after 6 and $24 \mathrm{~h}$.

Tablin et al. (2012) reported that of 14 investigated genes, the most massive increase of expression in the mouse airways and lung parenchyma by exposure to concentrated ambient particulate was Cyplal.
Igarashi et al. (2012) developed a mouse model humanized for the important xenobiotic sensor PXR (SXR) which controls the induction of several CYPs (CYP3A, CYP2B) and further xenobiotica-metabolizing enzymes (such as carboxylesterase 6). The mouse versus human PXR differ in their ligand-binding specificities. In the humanized mice CYP3A (Cyp3a11) mRNA was now induced by the humanspecific ligand rifampicin and no longer by the mouse-specific ligand pregnenolone-16 $\alpha$-carbonitrile. The successful expression of the humanized PXR was demonstrated for several organs including lung.

Chikara et al. (2018) showed a strong increase in the mRNA levels of CYP1A1, CYP2A5 and a strong decrease in the mRNA levels of CYP4F37 in mouse lung upon treatment with flaxseed.

Yun et al. (2017) reported for the mouse lung marked changes of CYP1A1 and 1B1 transcript levels upon cigarette smoke exposure.

Gibbs-Flournoy et al. (2018) showed that the exposure of mice to fumes emitted from cooking fires led to great (50fold) increases in lung CYP1A1 mRNA when traditional 3 -stone fire or advanced natural-draft stoves were used, whereas no increase was noted upon exposure of the mice to forced-draft stoves.

Bauer et al. (2018) showed in an in vitro murine lung epithelial cell model (the non-tumorigenic type II cell line C10) significant increases in CYP1B1 mRNA levels upon co-treatment with BP and a "low molecular-weight" PAH mixture (1-methylanthracene and fluoranthene).

\section{CYP protein expression}

Total CYP protein in the (Swiss albino) mouse lung was reported by Kalpana Deepa Priya et al. (2011) to amount to slightly less than $0.6 \mathrm{nmol} / \mathrm{mg}$ microsomal protein, total cytochrome b5 to slightly less than $0.4 \mathrm{nmol} / \mathrm{mg}$ microsomal protein. CYP but not cytochrome b5 protein was increased (almost doubled) by pretreatment of the animals with the (putative) anticarcinogen sulforaphane.

Using a monoclonal antibody raised against rat liver polycylic aromatic hydrocarbon-induced CYPs, staining of mouse lung was only seen after treatment of the mice with 3-methylcholanthrene (Forkert et al. 1986, 1989; Anderson et al. 1987). Using a monoclonal antibody which recognized CYP1A1 (called P1-450 in the original publication) staining was apparent in alveolar wall cells (most intensely in type II pneumocytes), but not in other mouse lung cells. Using a monoclonal antibody which recognizes both, rat CYP1A1 (called P-450 BNF/MC-B in the original publication) and also CYP1A2 (called P-450 BNF/ISF-G in the original publication) stained in addition to alveolar wall cells also the capillary endothelium (Forkert et al. 1989; Anderson et al. 1987). Jiang et al. (2009) reported that in mouse lung 
CYP1A1 protein was induced by treatment of the animals with 3-methylcholanthrene and that the induced activities were maintained for 15 days.

Using a monoclonal antibody raised against rat liver CYP2B2 (called P-450 PB-B in the original publications) staining of the mouse bronchiolar epithelium (highest in Clara cells) and of type II pneumocytes, but not of other mouse lung cells was observed by Forkert et al. (1986, 1989). Staining was essentially identical in untreated mice and after treatment with phenobarbital or 3-methylcholanthrene. Renaud et al. (2011) showed that of 14 mouse organs investigated CYP2B10 protein constitutive levels were very high in the male mouse lung, $600 \%$ higher than in the liver.

Baldwin et al. (2004) observed that CYP2F protein, which is converting the selective mouse pneumotoxin naphthalene to its reactive metabolite naphthalene 1,2-epoxide, is highly expressed in mouse lung. In the mouse kidney no CYP2F protein was found. The CYP2F protein levels in the mouse airways were 20- to 40-fold higher than in rat airways. In both rodent species the highest CYP2F protein levels within the lung were found in the distal airways. In the Rhesus Macaque no CYP2F protein was found in the airways.

CYP4B2 protein was relatively high in mouse lung microsomes (Imaoka et al. 1995).

Sadler et al. (2018) reported that in the mouse lung highfat diet did not significantly change the amount of any of the investigated CYPs protein (in contrast to the liver), but exposure to cigarette smoke increased all of them (CYP1A1, 1A2, 2a5, 22B10, 2B10, 2B19, 2D11, 2D26, 2F2, 2S1, 4B1, 4V2, 20A, 39A1).

Stoddard et al. (2018) performed a developmental protein profiling study in mouse lung. The protein of the lungselective CYP2F2 was present at postnatal day 0 , increased 11-fold until day 21 and stayed then static until day 42 . CYP2B10 was not detected until postnatal day 21 and thereafter showed no further increase until day 42. CYP4B1 protein was detected on gestational day 17 , postnatal days 21 and 42. Its levels increased between gestational day 17 and postnatal day 216 -fold.

\section{CYP catalytic activities (Table 3)}

Styrene Styrene is carcinogenic for the mouse lung, but not for the rat lung. Upon inhalation lung adenomas were observed starting at $20 \mathrm{ppm}$, carcinomas at $160 \mathrm{ppm}$ (Cruzan et al. 2001), while styrene was not tumorigenic for rats up to 1000 ppm (Cruzan et al. 1998). Styrene also proved carcinogenic for mice upon exposure via gavage (Ponomarkov and Tomatis 1978; U. S. NCI 1979). No increase of tumors have been found in eight chronic rat studies on styrene (discussed in Cruzan et al. 2002). CYP-catalyzed metabolism of the mouse lung is, therefore, of special interest and is singled out here in a special chapter, while the activities for various other compounds are treated in the subsequent chapter.

Since styrene was largely negative in tests on DNA reactivity, while species and organ specificity of its tumorigenicity largely coincide with the locations of its cytotoxicity (Cohen et al. 2002), a cytotoxicity-dependent mode of action is likely. For an early review of styrene's toxicity see Bond (1989), for a later comprehensive evaluation see Cohen et al. (2002).

The complex metabolism of styrene in mouse contrasted with other experimental animal species and man has been reviewed by Vodicka et al. (2006). Important data concerning mouse pulmonary CYP-mediated metabolism are detailed below (note that, according to the scope of this review, only the local metabolism in the lung is considered).

Initially, the CYP-dependent biotransformation of styrene to the electrophilically reactive styrene 7,8 -oxide was suspected to be the major reason for the pneumotoxicity and lung tumors produced in mice. Some of the findings supporting this suspicion and investigating the contribution of individual CYPs to this metabolism were as follows.

Mendrala et al. (1993) reported for B6C3F1 mouse lung microsomes a relatively high rate of pulmonary biotransformation of styrene to styrene glycol (rightfully presumed to be via styrene 7,8 -oxide) of $2.7 \mathrm{nmol} / \mathrm{min} / \mathrm{mg}$ protein and Carlson (1997) for CD-1 mouse lung microsomes a rate of $2.06 \mathrm{nmol} / \mathrm{mg}$ protein/min [much faster than the rate in human lung microsomes of $0.0065 \mathrm{nmol} / \mathrm{mg}$ protein $/ \mathrm{min}$ (Nakajima et al. 1994)]. Worthy of note in this context is that Carlson et al. (2000) detected only in one out of eight human lung samples any styrene metabolism $(0.088 \mathrm{nmol} /$ $\mathrm{min} / \mathrm{mg}$ protein) while all eight samples had CYP activities for benzene used as a control substrates. Similarly Filser et al. (2002) found no metabolism of styrene in human lung homogenates, while they actively metabolized 7-ethoxycoumarin. Concluding from these studies, the metabolic toxication in the human lung appears - in marked contrast to the susceptible species mouse- to be very low.

An important detail: differences in the metabolic hydrolysis of the electrophilically reactive styrene 7,8-oxide to the unreactive styrene glycol by pulmonary $\mathrm{mEH}$ between mouse strains does not explain their different susceptibility to styrene's pneumotoxicity (Carlson 1998).

With respect to individual CYPs involved in the metabolism of styrene, Carlson (1997) demonstrated that low concentrations of the CYP2E1 inhibitor diethyl dithiocarbamate inhibited the metabolism of styrene to styrene 7,8-oxide in mouse lung and liver microsomes, while the CYP2F2 inhibitor 5-phenyl-1-pentyne predominantly inhibited the styrene metabolism in the mouse lung microsomes.

Carlson et al. (1998) showed that the CYP1A inhibitor $\alpha$-naphthoflavone did not inhibit styrene metabolism in mouse lung (or liver) microsomes, implying that CYP1A 
is not important for the metabolism of styrene in the mouse lung (or liver). Similarly, the CYP2B inhibitor $\alpha$-methylb enzylaminobenzotriazole inhibited the formation of styrene 7,8-oxide by these preparations only slightly (16-19\% inhibition of styrene 7,8-oxide formation at $1 \mu \mathrm{M}$ inhibitor) (while causing a $87 \%$ inhibition of the hepatic metabolism of the CYP2B substrate 7-benzyloxyresorufin), implying that CYP2B also is not important for the biotransformation of styrene to styrene 7,8-oxide.

Hynes et al. (1999) observed that isolated mouse lung Clara (Club) cells, the target cells for styrene-induced pneumotoxicity and the presumed cells of origin of the lung tumors in mice, readily metabolized styrene to styrene 7,8oxide. This metabolism was inhibited by the CYP2E1 inhibitor diethyldithiocarbamate, but was much more efficiently inhibited by the CYP2F2 inhibitor 5-phenyl-1-pentyne, implying a role of CYP2E1, but a much more decisive role of CYP2F2. The CYP1A inhibitor $\alpha$-naphthoflavone did not inhibit and the CYP2B inhibitor $\alpha$-methylbenzylaminobenzo triazole only minimally inhibited styrene biotransformation to styrene 7,8-oxide in mouse Clara cells. Styrene metabolism was very efficiently catalysed by mouse Clara cells while mouse alveolar type II cells were almost inactive [in agreement with styrene-induced hyperplasia being observed in the terminal bronchioles, later extending to alveolar ducts, but not to alveolar cells (Cruzan et al. 1998, 2001)]. Styrene metabolism was severalfold higher in mouse Clara cells compared with Clara cells of the species resistant to the tumorigenic action of styrene, the rat (Hynes et al. 1999).

Moreover, using the styrene metabolism data of Hynes et al. (1999), Sarangapani et al. (2002) developed a PBPK model of styrene metabolism which predicts that in mouse bronchioles the more genotoxic and more cytotoxic enantiomer $(R)$-styrene 7,8-oxide (Gadberry et al. 1996) was preferentially formed, while in the rat lung the $(S)$-enantiomer is preferentially formed. In addition, the model predicts that, due to saturation of styrene metabolism in the rat lung at 500-600 ppm styrene, the maximally achievable concentration of $(R)$-styrene 7,8-oxide in rat terminal bronchioles is $2 \mu \mathrm{M}$, which is only half the level achieved in mouse terminal bronchioles at the lowest investigated mouse-carcinogenic dose of $20 \mathrm{ppm}$ styrene. The Filser team developed a PBPK model for the metabolism of styrene, initially based on the metabolism in the liver (Csanády et al. 1994), which they subsequently extended to also include local metabolism in the lung (Csanady et al. 2003). Using the latter model they calculated a predicted concentration in the human lung of $0.016 \mu \mathrm{mol}$ styrene 7,8 -oxide per $\mathrm{kg}$ body weight for humans exposed to $20 \mathrm{pm}$ styrene, 17 times lower than in the rat and 50 times lower than in the mouse.

Taken together, obviously the lung tissue of the animal species susceptible to the carcinogenic action of styrene, the mouse, produces more styrene 7,8-oxide than that of the resistant rat and the latter much more than the human lung tissue (Nakajima et al. 1994; Carlson et al. 2000; Filser et al. 2002) [in addition, the major styrene 7,8-oxide inactivation by the microsomal epoxide hydrolase proceeds faster in human than in the rat, and similar in the latter compared with the mouse, albeit the activity of the minor styrene 7,8oxide inactivating system, pulmonary GST, is much lower in human than in the rat, and lower in the rat than in the mouse (see in the respective chapters)].

However, several additional findings cast doubt that styrene 7,8-oxide could be the sole or the major entity responsible for the mouse lung tumors induced by styrene.

The first step in the major biotransformation pathway of styrene is formation of styrene 7,8-oxide in the lung-cancersusceptible mouse, but likewise in the resistant rat as well, this entire pathway accounting for at least $80 \%$ of the total styrene metabolism (Sumner and Fennell 1994; Cruzan et al. 2002).

Styrene-induced mouse pneumotoxicity and mouse lung tumors are not related to blood levels of styrene oxide (Cruzan et al. 2002) [it should, however, also be taken into account that Sarangapani et al. (2002) reported that in their PBPK model of styrene metabolism the predicted concentration of styrene 7,8-oxide specifically in the mouse terminal bronchioles correlated better with the tumorigenicity results of Cruzan et al. (2001) than the concentration of styrene 7,8-oxide in the blood].

Using the PBPK model developed by Filser et al. (1999) the following was observed: The predicted concentration of styrene 7,8-oxide in the mouse lung upon inhalation of $40-80 \mathrm{ppm}$ styrene is virtually not different from the concentration in the rat lung exposed to $1000 \mathrm{ppm}$. The former concentration had led to mouse lung tumors, while the latter concentration was not tumorigenic (Cohen et al. 2002). Also, when considering the more toxic isomer $(R)$-styrene 7,8 -oxide, the predicted difference of its concentration in the mouse versus the rat lung ( $\sim 2$-fold) is not sufficient to explain the species difference in the styrene-induced tumorigenicity (Cohen et al. 2002) [the Harvard Center for Risk Analysis (HCRA) potential health risks associated with exposure to styrene review panel (Cohen et al. 2002) warned to give much weight to these modeled data because of inconsistencies in the measured levels of styrene 7,8-oxide in the blood]. Furthermore, oral administration of styrene doses leading to higher styrene 7,8-oxide levels in the lung of rats than those which have led to lung tumors in mice did not lead to tumors in rats (Cruzan et al. 2009).

In ventilated, perfused lungs of mice and rats exposed to styrene vapours the concentration of styrene 7,8-oxide in the effluent perfusate was only 2 -fold different, far too small to account for the species difference of susceptible versus resistant to the tumorigenicity of styrene (Hofmann et al. 2006). 
Ring-oxidized styrene metabolites (4-vinylphenol and/or metabolites derived from it) were shown to be important for styrene's pneumotoxicity, specifically the toxicity to Clara (Club) cells, the presumed site of origin of the mouse lung tumors induced by styrene (Carlson et al. 2002; Cruzan et al. 2005). 6-60 mg/kg/day for 14 days produced cytotoxicity in the terminal bronchioles of mice, but not of rats (which are resistant to the styrene-induced tumorigenicity).

4-Vinylphenol was 10 times as toxic as the parent compound styrene and 5 times as toxic as styrene 7,8-oxide (Carlson et al. 2002).

Kaufmann et al. (2005) showed that the intraperitoneal treatment of mice with the side chain styrene metabolites phenylacetaldehyde and phenylacetic acid caused apoptosis and cell proliferation only in the alveoli, but no effects in the terminal bronchioli, the presumed site of origin of the styrene-induced mouse lung tumors. However, treatment with styrene 7,8-oxide at a relatively high dose of $3 \times 100 \mathrm{mg} /$ $\mathrm{kg}$ body weight per day or with 4-vinylphenol already at a relatively low dose of $3 \times 20 \mathrm{mg} / \mathrm{kg}$ body weight per day led to an up to 19-fold increase in cell proliferation, glutathione depletion and histomorphological changes in the large/medium bronchi and terminal bronchioli including a loss of the typical bulging of the dome-shaped Clara cells. Histomorphologically, the damage was confined to the nonciliated bronchiolar epithelium with a severe initial damage to the Clara cells and the highest increase of cell proliferation occurred in the terminal bronchioli. These results suggest that 4-vinylphenol and/or their metabolites and-with lower potency-also styrene 7,8-oxide are the causative entities for the styrene-induced toxicity-dependent tumorigenicity of styrene for the mouse lung.

The data reported by Sumner and Fennell (1994) and Johanson et al. (2000) show by whole-body metabolism studies that mice produce much more styrene-derived ringoxidation products than rats.

Inhibition of CYP2F2 activity by 5-phenyl-1-pentyne prevented largely (up to practically 100\%) the cytotoxicity of 4-vinylstyrene in the mouse lung (Carlson 2002), demonstrating that not 4-vinylphenol itself, but rather a CYP2F2dependent metabolite derived from it, is a highly pneumotoxic agent.

4-Vinylphenol, is - in presence of NADPH-rapidly further metabolized by lung microsomes of the susceptible species, the mouse, and the resistant species, the rat, but by the mouse lung microsomes much ( 8 -fold) faster than by rat lung microsomes. The major catalyst for this biotransformation step in the mouse lung is CYP2F2. Lack of UV absorbance indicated that the metabolite(s) was/were ring-opened derivative(s) of styrene (Cruzan et al. 2002).

Green et al. (2001b) reported that inhibition of CYP2F enzymatic activity by the selective CYP2F inhibitor 5-phenyl-1-pentyne drastically reduced the styrene-induced increases in cell toxicity/necrosis and subsequent cell divisions in mouse lung bronchioles.

In whole-animals, namely CYP2E1 knock-out mice, pneumotoxicity of styrene was similar to that observed in wild-type mice, in contrast to the liver, where toxicity in the CYP2E1 knock-out mice was much reduced compared with the wild-type mice, demonstrating that for styrene metabolic toxication in mouse liver CYP2E1 was important, but not in lung. The author concludes that the results support the idea that for pneumotoxicity of styrene CYP2F2-dependent metabolism of styrene is important, but that for the susceptibility to the intrinsic toxicity of the reactive metabolite, styrene 7,8-oxide, there is no difference whether CYP2E1 activity is present or not (Carlson 2004).

CYP2Fs in various animal species appear to possess in general similar substrate preferences, but not for styrene: CYP2F2 of mice and CYP2F4 of rats readily metabolize styrene, but not the human CYP2F1 (Cruzan et al. 2002; Carlson 2008), e.g. Green et al. (2001a) did not detect styrene metabolism in the human nasal tissue, which possesses CYP2F1. While the mouse CYP2F2 and the rat 2F4 are structurally similar, the human CYP2F1 differs distinctly in having in its active site a lysine substituted for glutamine (Lewis et al. 2009). In this context it is of interest to note that the styrene metabolite and proximal pneumotoxin 4-vinylphenol (Carlson 2002) has been reported to be present (as a minor metabolite) in the urine of styrene-exposed workers (Pfaffli et al. 1981), but the CYP-dependent activity required for the toxication of 4-vinylphenol was not detected in the human lung (see above). In contrast to the findings of Pfaffli et al. (1981), Johanson et al. (2000) failed to detect in exhaled air, blood or urine of volunteers exposed to ${ }^{13} \mathrm{C}$-styrene vapour metabolites which could have been derived from styrene ring oxidation and Cruzan et al. (2002) concluded that in human urine ring-oxidized styrene metabolites were below detection.

Comparing potential styrene metabolism in mouse and rat Clara (Club) cells and type II alveolar pneumocytes Hynes et al. (1999) and Carlson et al. (2000) showed that styrene metabolism is virtually completely restricted to Clara cells [consistent with the localization of CYP2F in Clara cells and not in type II alveolar pneumocytes (Forkert 1995; Buckpit et al. 1995)] and is much faster in mice compared with rats.

Mice Clara cells possess 30-fold higher levels of CYP2F than rat Clara cells, while the lungs of the primate rhesus macaque has no measurable CYP2F (Baldwin et al. 2004). In this context it is worthy of note that the human lung possesses very few Clara cells and, moreover, the human Clara cells have very little to no smooth endoplasmic reticulum and very low levels of xenobiotica-metabolizing CYPs (Gram 1997; Ding and Kaminsky 2003).

In CYP2F1 knock-out mice Clara cell toxicity and general terminal bronchiole toxicity were not seen upon exposure to 
styrene or to styrene 7,8-oxide, whereas the wild-type mice treated with the same doses showed very marked necrosis and exfoliation of Clara cells as well as a strong increase of cell division in the terminal bronchioles (Cruzan et al. 2012).

Comparing CYP2E1-null mice with CYP2F2-null and wild-type mice, Shen et al. (2014) found neither a significant difference between 2E1-null and wild-type mice of styrene metabolism in lung microsomes [formation of styrene glycol (via styrene 7,8-oxide) and formation 4-vinylphenol] nor of styrene-induced lung toxicity (cell counts and LDH activity in bronchoalveolar fluid), while in 2F2-null mice lung microsomes metabolic formation of styrene glycol and 4-vinylphenol were dramatically reduced and styrene did not lead to pneumotoxicity.

Taken together the available data on styrene metabolic toxication in the mouse lung, it appears obvious that

- the CYP forms predominantly responsible for toxication of styrene in the mouse lung are different from those in the mouse liver: CYP2F2 in the lung, CYP2E1 in the liver;

- ring-oxidized metabolites of styrene are important for the styrene-induced pneumotoxicity in mice;

- mouse lung produces much more ring oxidation of styrene than the rat lung, while styrene ring oxidation in human lung is almost nil;

- the CYP form CYP2F2 is predominantly or exclusively responsible for the styrene-induced mouse-selective pneumotoxicity and lung tumors, the former centred in the bronchioles, the latter in the same region, albeit not distinguishable whether bronchiolar or alveolar or both;

- the CYP form homologous to the mouse CYP2F2 in the rat is CYP2F4, in human 2F1. Mouse lung possesses much higher CYP2F-dependent catalytic activity than the rat lung and the latter much more than the human lung.

CYP catalytic activities for compounds other than styrene Lorenz et al. (1984) showed that mouse lung microsomes very efficiently catalysed the deethylation of the broad-spectrum CYP substrate 7-ethoxycoumarin, almost 10 -fold faster than rat lung microsomes and about 1000-fold faster than human lung microsomes. Rates of 7-ethoxycoumarin O-deethylation were much higher in mouse Clara (Club) cells compared with mouse alveolar type II cells (Devereux et al. 1989).

Jiang et al. (2009) reported that in mouse lung CYP1A1/1A2-selective EROD activity was present, massively (380-fold) induced by treatment of the animals with 3-methylcholanthrene and that the induced activities were maintained for 15 days after discontinuation of the treatment.
Paolini et al. (1995) reported the presence of CYP2Bdependent PROD activity in the mouse lung and its inducibility by quite a number of compounds. Interestingly, of all compounds tested, sodium barbital led to the highest enhancement of PROD activity in the mouse lung (46-fold and 50-fold in the male and female mice, respectively), rather than sodium phenobarbital (2.5-fold and 8-fold in male and female mice, respectively). Phorone and trans1,2-dichloroethylene also led to increases of PROD activity in the mouse lung.

The CYP2E1-prototypic 4-nitrophenol hydroxylation was observed in mouse lung microsomes by Forkert et al. (2000).

Canistro et al. (2009) observed in mouse lung microsomes quite a number of CYP-mediated catalytic activities (EROD, MROD, PROD, testosterone hydroxylations at seven different positions) and that all of them were decreased upon treatment of the mice for 7 days with $50 \mathrm{mg} / \mathrm{kg}$ body weight of the (putative) health promoter resveratrol (the treatment was i.p., according to the authors in order to avoid a possible confounding factor by a first pass effect upon oral treatment).

Buckpit et al. (1995) noticed active and CYP2F2-associated metabolism of naphthalene in the airways of the species mouse, which is sensitive to its selective pneumotoxicity, while much slower metabolism of naphthalene was noticed in the airways of the rat, which is a species resistant to its toxicity. While CYP2F-dependent metabolism of naphthalene is especially crucial for its pneumotoxicity, several other CYPs have been shown to also contribute to the biotransformation of naphthalene to its reactive metabolite 1,2-epoxide: CYP1A1 (Greene et al. 2000), CYP1A2 (Cho et al. 2006), CYP2A5/6 (Asikainen et al. 2003), CYP2B (Nagata et al. 1990; van Bladeren et al. 1985) and CYP2E1 (Wilson et al. 1996). However, Genter et al. (2006) provided strong suggestive evidence that CYP1A1 and 1A2 do not significantly contribute to naphthalene bioactivation in vivo.

Genter et al. (2006) observed that the CYP2F inhibitor 5-phenyl-1-pentyne abolished the olfactory toxicity of naphthalene in mice, which suggests a decisive role of CYP2F-mediated metabolism of naphthalene for its toxicity in mice. Li et al. (2011b) showed that in Cyp2f-null mice the in vitro catalytic efficiency of lung microsomes for the epoxidation of naphthalene was decreased 160 -fold, but in the nasal mucosa only 16 -fold, and consequently the Cyp $2 f$ null mice were largely resistant against the pulmonary toxicity of naphthalene, but not against its cytotoxicity toward the nasal mucosa.

Stereochemical studies showed that mouse lung converted naphthalene with high preference to the $1 R, 2 S$-naphthalene epoxide in contrast to the rat lung. However, studies on the fate of the enantiomers strongly suggested that the rate of the epoxide formation rather than differences between the epoxide enantiomers are related to the species differences in 
susceptibility to the toxicity of naphthalene (Buckpitt et al. 2002).

Shultz et al. (1999) found that in the metabolism of the species-selective pulmonary toxicant naphthalene mouse CYP2F2 (107 nmol/nmol CYP/min) was 2400 times faster than human CYP2F1 (0.045 nmol/nmol CYP/min) and Shultz et al. (2001) found that the pulmonary toxicants 1-nitronaphthalene and 2-methylnaphthalene are metabolized by recombinant mouse lung-selective CYP2F2 with high $k_{\text {cat }}$ values (17.1 and 3.7 $\mu \mathrm{M}$, respectively). All of this suggests that mouse CYP2F2 is highly important for the selective murine pneumotoxicity of several pneumotoxins.

The mutagenic nitroaromatic compound 1-nitronaphthalene (emitted from diesel engines) was shown to be activated by the mouse lung CYPs (Watt and Buckpitt 2000), the rapid metabolism by mouse lung was confirmed by Baldwin et al. (2005).

The rodent carcinogen butadiene is metabolized by CYP isoenzymes, most notably including CYP2E1 and CYP $3 \mathrm{~A} 4$, to at least two genotoxic metabolites, butadiene epoxide and diepoxybutane (Bond and Medinsky 2001). It was converted more than 10 -fold faster to the monoepoxide by lung microsomes of the much more sensitive species mouse compared with lung microsomes of the much less sensitive species rat. Diepoxide formation also occurred in mouse lung microsomes ( $V_{\max } 0.2 \mathrm{nmol} / \mathrm{mg}$ protein $/ \mathrm{min}$ ), whereas this biotransformation did not occur at a measurable rate in rat or human lung microsomes (Csanády et al. 1992). Seaton et al. (1996) observed that isolated bronchioles of mice, which upon exposure to butadiene develop brochiolar-alveolar neoplasms, metabolized butadiene to the reactive butadiene epoxide twice as fast as rats, which are much less susceptible.

Hellmold et al. (1993) observed in mouse lung microsomes metabolic activation of the aromatic amines IQ (2-amino-3,8-dimethylimidazo[4,5-f]quinoline) and MeIQx (2-amino-3,8-dimethylimidazo[4,5-f]quinoxaline) to derivatives mutagenic for Salmonella typhimurium TA98. $\alpha$-Naphthoflavone, reputed to be a specific inhibitor of CYP1A activity, inhibited the activation of IQ by $30-40 \%$ and the activation of MeIQx by about $60-80 \%$ (however, the authors speculated that this inhibition may rather have been due to a hitherto unknown cross-inhibition of CYP2A3 activity by these antibodies).

Thornton-Manning et al. (1996) showed that the lung toxin 3-methylindole was efficiently activated to the reactive 3-methylindoleimine by recombinant mouse CYP1A2, in amounts exceeding those produced by the human CYP most active in this respect, CYP2F1. However, Zhou et al. (2012) showed by comparison of CYP2F2 knock-out mice with CYP2A5 knock-out and with wild-type mice, that mouse lung CYP2F2 preferentially metabolized 3-methylindole to reactive iminium ions.
Price et al. (1996) used mouse lung slices to show that agaritine was biotransformed by mouse (and rat) lung to reactive metabolites, which covalently bound to lung proteins, more in the mouse lung compared with the rat lung.

Green et al. (1997) found that metabolism of trichloroethylene was fast in mouse lung (23-fold slower in rat lung and not detectable in human lung).

The pneumotoxin and lung carcinogen ethyl carbamate was metabolically activated by mouse lung CYP2E1 (Forkert and Lee 1997), the activity of which is about 7-fold higher in the mouse lung compared with the human lung.

The Clara (Club) cell pneumotoxin 1,1-dichloroethylene was shown to be toxified by the mouse lung CYP2E1 to the reactive epoxide (Forkert 1999) and higher levels of the epoxide-derived glutathione conjugate were shown to be formed in the mouse lung compared with the human lung (Dowsley et al. 1999).

Born et al. (2002) used the CYP2F inhibitor 5-phenylpentyne to show that in the mouse lung CYP2F2 is the major catalyst responsible for the conversion of the selective mouse pneumotoxin coumarin to its 3,4-epoxide, the metabolite (and/or its rearrangement product ortho-hydroxyphenylacetaldehyde) responsible for coumarin's selective toxicity for the mouse lung (while coumarin 7-hydroxylation is the major pathway and coumarin 3-hydroxylation is a minor pathway in mouse, rat and human). Inhibition of CYP2F2 activity using 5-phenyl-1-pentyne eliminated coumarin's bronchiolar cytotoxicity.

\section{Non-CYP oxidoreductases (Table 4)}

\section{Flavin-dependent monooxygenases (FMO)}

Falls et al. (1997) observed FMO3 mRNA in mouse lung (of both sexes, but in the liver only in males and in the kidney not in either sex).

Sainkhuu et al. (2016) reported the presence of FMO2, FMO3 and FMO4 mRNA in the murine lung as well as its increase upon oral treatment of mice with Phellinus baumii extract and also by its glucan fraction ( $P$. baumii is a mushroom long used in the alternative medicine of Korea and other Asian countries).

In a developmental study on mouse lung Stoddard et al. (2018) found FMO1 protein in one sample on postnatal day 0 and then in all samples on postnatal days 21 and 42 . FMO2 protein was detected at all time points between gestational day 17 to postnatal day 42 , with a total 29 -fold increase.

\section{Cyclooxygenase (COX)}

Cox convert arachidonic acid to prostaglandins. In the first step a hydroperoxide cyclic endoperoxide is formed (prostaglandin $\mathrm{G}_{2}$ ), in the second step the hydroperoxide function 
is reduced to hydroxyl, leading to prostaglandin $\mathrm{H}_{2}$. Xenobiotica of sufficient lipophilicity and sufficient low redox potential (many phenols and aromatic amines) can serve as oxygen acceptor/electron donor in this second step. Many xenobiotica can be toxified by this co-oxidation reaction, e.g. forming unpaired electron radicals which may react with further molecules generating free radicals from them and ultimately lead to toxicity including lipid peroxidation as well as formation of DNA adducts.

Bauer et al. (2018) showed in an in vitro murine lung epithelial cell model (the non-tumorigenic type II cell line C10) significant increases in COX-2 mRNA levels upon co-treatment with BP and a "low molecular-weight" PAH mixture (1-methylanthracene and fluoranthene).

Gibbs-Flournoy et al. (2018) showed that the exposure of mice to fumes emitted from cooking fires led to significant increases in lung COX-2 mRNA when traditional 3-stone fire or advanced natural-draft stoves were used, whereas no increase was noted upon exposure of the mice to forceddraft stoves.

Rioux and Castonguay (1998) provided strong indirect suggestion that via inhibition of COX, especially COX-2, in the mouse lung a marked reduction of NNK metabolic bioactivation is achieved and that consequently this leads to a marked inhibition of NNK-mediated carcinogenesis in the mouse lung. Acetylsalicylic acid was used as a nonspecific COX inhibitor, $N$-[2-(cyclohexyloxy)-4-nitrophenyl]-methanesulfonamide (NS-398) as a selective COX2 inhibitor.

\section{NADH/NADPH quinone oxidoreductase (NQO)}

Transcripts Vanhees et al. (2012) reported the presence of NQO1 mRNA in mouse lung and a major (11.3-fold) increase in its level in 12-week-old male offspring after prenatal exposure to quercetin. However, no increase was observed in female offspring.

Zhang et al. (2012) observed increased levels of NQO1 mRNA in young (3-month-old), but not in middle-aged 18 -month-old) mouse lung after chronic $(150 \mathrm{~h}$ over 10 weeks) exposure to "vehicular-derived airborne nanosized particulate matter".

Yun et al. (2017) reported for the mouse lung a marked increase of NQO1 transcript formation by cigarette smoke exposure.

Gibbs-Flournoy et al. (2018) showed that the exposure of mice to fumes emitted from cooking fires led to 10-fold increases in lung NQO1 mRNA when traditional 3-stone fire or advanced natural-draft stoves were used, whereas no increase was noted upon exposure of the mice to forceddraft stoves.

Enzymatic activity Kalpana Deepa Priya et al. (2011) reported that mouse lung cytosol possesses NQO activ- ity, which is neither influenced by pretreatment of the animals with BP nor with the (putative) anticarcinogen sulforaphane.

\section{Carbonyl reductase (CBR)}

CBR catalyzes the reduction of the carbonyl function to the hydroxyl function and the reverse reaction. Substrates include prostaglandins and various xenobiotics. NAD(P) (H) serve as cofactor.

In a developmental study on mouse lung Stoddard et al. (2018) found CBR2 protein from gestational day 17 to postnatal day 42 with a total increase of 42 -fold [CBR2 is thought to be selectively expressed in the lung (Nakanishi et al. 1995).

Yun et al. (2017) reported for the mouse lung the CBR3 transcript formation and its marked increase by cigarette smoke exposure.

\section{Alcohol dehydrogenase (ADH) and aldehyde dehydrogenase (ALDH)}

Alcohol dehydrogenase (ADH) Stoddard et al. (2018) reported that in the developing mouse lung the alcohol dehydrogenases ADH1, ADHX (primarily involved in the dehydrogenation of long-chain primary alcohols)and AK1A1 (primarily involved in the reduction of aldehydes) proteins were present at gestational day 17 and decreased 3-fold until the last investigated time point of postnatal day 42 .

Aldehyde dehydrogenase (ALDH) Tablin et al. (2012) reported that of 14 investigated genes the second most massive increase of expression in the mouse airways by exposure to concentrated ambient particulate matter was Aldh3al (second after Cyplal).

Yun et al. (2017) reported for the mouse lung the ALDH3A1 transcript formation and its marked increase by cigarette smoke exposure.

Individual ALDHs developed in the mouse lung differently. ALDH1A1 protein increased from gestational day 17 to postnatal day 425 -fold while ALDH1A7 protein decreased 5-fold during the same period of time (Stoddard et al. 2018).

\section{Hydrolases}

\section{Epoxide hydrolase (EH)}

Microsomal epoxide hydrolase ( $\mathrm{mEH}$, also called EPHX1) (Table 3) Early on Oesch et al. (1977) investigated mEH activities in six extrahepatic organs of the mouse. Specific 
activities were remarkably high in the lung, third after liver and testis.

With regard to inactivation of the genotoxic styrene metabolite styrene 7,8-oxide the data in Oesch et al. (1977) and in Oesch et al. (1980) taken together show that microsomal epoxide hydrolase activity in the mouse lung is similar to that in the rat lung and the latter much lower than in the human lung. The data were obtained in part using styrene 7,8-oxide as substrate and in part using BP 4,5-oxide as substrate. However, Oesch and Bentley (1976) had shown that a single enzyme is catalyzing the hydrolysis of both, styrene 7,8-oxide and BP 4,5-oxide.

Boogaard and Bond (1996) reported that mouse lung microsomes hydrolyzed diepoxybutane with a much lower $V_{\max } / K_{\mathrm{m}}$ efficiency (3.93) than the rat (19.2) and human (32.5) providing a likely contribution for the much higher susceptibility to the carcinogenicity of the parent compound butadiene of the mouse lung compared with the rat lung.

In order to find a possible reason for the fact that the neonatal lung of various experimental animal species, including mice (Fanucchi et al. 1997), is especially vulnerable in response to cytotoxicants although potentially toxifying CYP activities are lower than in adults, Fanucchi et al. (2000) investigated the perinatal development of the predominantly detoxifying phase II xenobiotic metabolizing enzymes $\mathrm{mEH}$ and GST in mice lung. Protein levels of pulmonary $\mathrm{mEH}$ (determined in proximal and in distal airways of fetal and postnatal mice) increased with increasing age of the mice. However, $\mathrm{mEH}$ activity (determined in the distal airways of postnatal mice using cis-stilbene oxide as substrate) did not change with increasing age.

Carlson (1998) showed that differences in the rates of $\mathrm{mEH}$-catalyzed detoxication of styrene 7,8-oxide by its conversion to the resulting glycol between NSA (non-Swiss albino), a mouse strain susceptible to styrene pneumotoxicity versus Swiss CD-1, a more resistant strain, is not the underlying mechanism for the different susceptibilities. He also reported that conversion of styrene 7,8-oxide to glycol was induced by butylated hydroxylanisole in the mouse liver, but not in the lung.

Soluble epoxide hydrolase (sEH, also called EPHX2) sEH was observed in the mouse lung where its specific activity was $1 / 15$ th of that in the mouse liver (Gill and Hammock 1980).

Zheng et al. (2001) showed that sEH protein was highly localized to the vascular smooth muscle of small and medium-sized pulmonary vessels. They showed that leukotoxin is actually a protoxin which is activated by sEH to the ultimately toxic diol causing ARDS (acute respiratory distress syndrome), massive alveolar oedema and haemorrhage with interstitial oedema around blood vessels in the lung of the treated mice.
Keserü et al. (2008) reported that sEH is localized in mouse bronchioles and in the smooth muscles which surrounds arterioles. Freshly isolated and cultured mouse lung vascular smooth muscle cells showed sEH activity.

$\mathrm{EH}_{3}$ The team of Michael Arand discovered a third epoxide hydrolase, which they called $\mathrm{EH}_{3}$ (Decker et al. 2012). Expression on the mRNA level in the mouse lung was high, third after stomach and skin, of totally 20 investigated organs, while the expression in the liver was extremely low.

Recombinant $\mathrm{EH}_{3}$ had a high turnover rate for 9,10-epoxy stearate, for endogenous, arachidonic acid-derived epoxides (8,9-11,12- and 14,15-epoxyeicostrienoic acid) and for leukotoxin (9,10-epoxyoctadec-11-enoic acid) (the highest specific activity for hydrolysis of arachidonic epoxides and for leukotoxin reported to-date). This suggests that EH3 substantially contributes to the toxicity of leukotoxin, since the hydrolytically generated metabolite is a strong mediator of acute respiratory distress syndrome. On the other hand, the hydrolytic activity toward a standard EH substrate, styrene 7,8-oxide (a substrate for both, $\mathrm{mEH}$ and $\mathrm{sEH}$ ), was extremely low (essentially nil) (Decker et al. 2012).

\section{Conjugating enzymes}

\section{Glutathione S-transferase (GST) (Table 6)}

Transcripts Mainwaring et al. (1996) demonstrated the presence of GST theta transcripts and protein in the mouse lung, especially in Clara (Club) cells. Levels were substantially higher than in the rat and human lung.

Vanhees et al. (2012) reported the presence of GSTP1 mRNA in mouse lung and a 2.2-fold increase in its level in 12-week-old male (but not female) offspring after prenatal exposure to quercetin.

Yun et al. (2017) reported for the mouse lung the GSTP1 and GSTO1 transcript formation and its marked decrease by cigarette smoke exposure in C57/BL6 mice.

Protein McLellan et al. (1992) reported that in mouse lung GSTs were localized exclusively to the bronch.

In order to find a possible reason for the fact that the neonatal lung appears to be especially vulnerable in response to cytotoxicants, Fanucchi et al. (2000) investigated the perinatal development of the predominantly detoxifying phase II xenobiotic metabolizing enzymes $\mathrm{mEH}$ and GST in mice lung. Development of GST protein expression differed between individual GST isozymes. GST alpha and GST mu protein levels (determined in foetal total lung, in postnatal mice in proximal and distal airways) were low before birth, high on postnatal day 7 , decreased between days 14 and 21 and became again high on day 28 and in adult mice. GST pi protein levels, 
however, were high on gestational day 18 , again on postnatal day 4 , became undetectable on day 21 , but were high again in adult mice. GST activity determined in the postnatal mouse distal airways cytosolic fraction using CDNB (1-chloro-2,4-dinitrobenzene) as substrate increased steadily from day 7 to adulthood. Thus, GST activity toward the broad-spectrum substrate CDNB does not correlate with the development of the expression of the major GST proteins alpha, mu and pi.

Enzymatic activity Autrup et al. (1980) showed in cultured mouse tracheobrochial tissue a high proportion of BP metabolized to glutathione metabolites, much more than to glucuronides.

Boogaard et al. (1996) observed considerable GST activity toward diepoxybutane in mouse lung cytosol $(38.5 \pm 2.5 \mathrm{nmol} / \mathrm{min} / \mathrm{mg}$ cytosolic protein), approximately twice the activity observed in the rat lung $(17.1 \pm 3.0 \mathrm{nmol} /$ $\mathrm{min} / \mathrm{mg}$ cytosolic protein) and concluded that differences between GST activities are not the reason for the mice upon exposure to butadiene accumulating higher concentrations of diepoxybutane in the blood than the rats, which are much more resistant to the carcinogenicity of butadiene.

With respect to inactivation of the reactive styrene metabolite styrene 7,8-oxide by GST the data summarized by Cohen et al. (2002) show that the activity is much higher in the mouse than in the rat and much higher in the rat than in human. Accordingly, Delbressine et al. (1981) had shown that the excretion of styrene 7,8-oxide-derived mercapturic acids were higher in rodents compared with humans.

Canistro et al. (2009) observed in mouse lung cytosol GST activity toward the broad-spectrum substrate CDNB which was substantially reduced upon treatment of the mice with the (putative) health promoter resveratrol (the treatment was i.p., according to the authors in order to avoid a possible confounding factor by a first pass effect upon oral treatment).

Li et al. (2011a) observed in mouse pulmonary cytosol GST activity toward ethylene oxide as substrate. Zheng et al. (2014) observed that GST specific activity toward the prototypic substrate 4-hydroxynonenal was very high in mouse lung $9000 \mathrm{~g}$ supernatant, almost as high as in the liver.

\section{UDP-glucuronosyltransferase (UGT) (Table 7)}

Transcripts Sivils et al. (2013) reported low levels of UGT1A mRNA and much higher levels of UGT2B mRNA in mouse lung (and a massive reduction of both of them in Mrp1 knock outs).

Vanhees et al. (2012) reported the presence of UGT1A6 mRNA in mouse lung and a 2.2-fold increase in its level in 12-week-old male (but not in female) offspring after prenatal exposure to quercetin.
Yun et al. (2017) reported for the mouse lung the UGT1A6 and 1A7 transcript formation and its marked decrease by cigarette smoke exposure in C57/BL/6 mice.

Chikara et al. (2018) showed a strong increase in the mRNA levels of UGT2B34 in mouse lung upon treatment with flaxseed.

Catalytic activity Canistro et al. (2009) observed in mouse lung microsomes UGT activity toward 1-naphthol as substrate which was substantially reduced upon treatment of the mice with the (putative) health promoter resveratrol (as stated above, the treatment was i.p., according to the authors in order to avoid a possible confounding factor by a first pass effect upon oral treatment).

Kalpana Deepa Priya et al. (2011) reported that mouse lung microsomes possess quite remarkable $(\sim 10 \mathrm{nmol} /$ $\mathrm{min} / \mathrm{mg}$ microsomal protein) UGT activity toward 4-nitrophenol as substrate, which is slightly (but statistically significantly) reduced by pretreatment of the animals with BP or with the (putative) anticarcinogen sulforaphane.

\section{Sulfotransferase (SULT)}

Sivils et al. (2013) reported the presence of SULT1A1 mRNA in mouse lung (and its massive reduction in Mrp1 knockouts).

Autrup et al. (1980) showed in cultured mouse tracheobrochial tissue a high proportion of BP metabolized to sulfates, much more than to glucuronides.

\section{$N$-Acetyltransferase (NAT)}

Dairou et al. (2009) observed in the mouse lung mtCC1-2 Clara (Club) epithelial cell line (developed by Magdaleno et al. 1997) NAT2 protein and enzymatic activities for the following substrates: benzidine, $\beta$-naphthylamine, 4-aminobiphenyl, $p$-aminosalicylic acid and 2-aminofluorene.

\section{Xenobiotica-metabolizing enzymes in the rabbit lung}

\section{Cytochromes P450 (CYP)}

\section{CYP transcript expression}

CYP4B 1 mRNA expression was considerably higher in rabbit lung compared with other tissues including liver (Zeldin et al. 1995). 


\section{CYP and related proteins expression}

Serabjit-Singh et al. (1980) observed CYP reductase protein in rabbit lung Clara (Club) cells. CYP reductase is more highly localized to rabbit lung Clara cells than to type II pneunmocytes (Devereux and Fouts 1981; Domin et al. 1986; Serabjit-Singh et al. 1988) which in turn contain higher levels of this enzyme than alveolar macrophages (Domin et al. 1986). No CYP reductase protein was observed in rabbit type I pneumocytes or endothelial cells (Serabjit-Singh et al. 1988).

Neither CYP1A1 nor CYP1A2 protein (in the original publications called form 6 and form 4, respectively) were detected constitutively in rabbit lungs, but they were highly induced by TCDD (Dees et al. 1982; Domin et al. 1986; Guengerich 1990) in bronchial and bronchiolar epithelia as well as in pulmonary artheria, veins and capillary endothelia (Dees et al. 1982). Low levels of CYP1A1 were detected in rabbit alveolar macrophages (Domin et al. 1986).

CYP2B4 (in the original work called cytochrome P-450 form 2) was observed in rabbit Clara cells and type II pneumocytes (Serabjit-Singh et al. 1980; Devereux and Fouts 1981) and in bronchial and bronchiolar epithelia and even in type I pneumocytes (Dees et al. 1982), the latter not confirmed by Serabjit-Singh et al. (1988). The relative levels of CYP2B4 were found to be high in Clara cells and considerably lower in ciliated epithelial cell of the bronchus and the bronchioli (Plopper et al. 1987; Serabjit-Singh et al. 1988). Location of CYP2B4 in capillary endothelial cells was observed by Serabjit-Singh et al. (1988), but not by Dees et al. (1982) (possible reasons for the discrepancy: (a) different antibodies were used in the two studies, (b) Dees et al. used light microscopy in frozen, unfixed specimens, while Serabjit-Singh used electron microscopy in fixed samples). Low levels of CYP2B4 were detected in rabbit alveolar macrophages (Domin et al. 1986).

CYP4B1 (in the original publication called form 5) was observed in highest levels in Clara cells, in lower levels also in ciliated cells of the bronchus and of the bronchioli, in type II pneumocytes and in capillary endothelial cells, but not in type II pneumocytes (Serabjit-Singh et al. 1980, 1988; Devereux and Fouts 1981; Plopper et al. 1987). Wolf et al. (1978) calculated that CYP4B1 protein amounted to at least $35 \%$ of total rabbit lung CYP proteins. Low levels of CYP4B1 were detected in rabbit alveolar macrophages (Domin et al. 1986). A second CYP4B protein (CYP4B4) was also observed in rabbit lung and determined to be present in a similar amount as CYP2B1 (Wolf et al. 1978).

\section{CYP catalytic activities (Table 3)}

The broad-spectrum CYP substrate 7-ethoxycoumarin was deethylated by rabbit Clara cells and by rabbit alveolar type
II cells, the rate of deethylation being higher in the former compared with the latter (Devereux et al. 1989).

Wheeler et al. (1990) characterized in human and baboon lung in comparison with rat and rabbit a CYP homologous to the toxicologically important CYP1A1 of rodents. Typical CYP1A enzymatic activities were found in human and baboon lung microsomes, in similar activities between these two species, but considerably lower activities as compared with rat and rabbit microsomoses (see Table 3 comparing the Wheeler et al. 1990 data between these species).

Zeldin et al. (1995) observed in rabbit lung microsomes complete inhibition by anti-CYP2B4 antibodies of arachidonic acid biotransformation to dihydroxyeicosatrienoic acid and to epoxyeicosatrienoic acid, but no inhibition of arachidonic acid metabolism by anti-CYP4B1 antibodies.

CYP-catalyzed monooxygenation of several xenobiotics have been observed in rabbit lung (Sabourin et al. 1988). CYP 2B4-dependent activities were much greater in Clara cells compared with type II pneumocytes [consistent with the selective toxicity of 4-ipomeanol for Clara cells, 4-ipomeanol being (additionally to CYP4B1) toxified by CYP2B4 (and the rat ortholog CYP2B2) (Guengerich 1977a, b)].

Williams et al. (1984) showed that rabbit lung CYP4A1 (called P450PG- $\omega$ in the original publication) hydroxylated arachidonic acid at its terminal carbon atom (omega-hydroxylase).

Robertson et al. (1981) demonstrated toxication by the $16,000 \mathrm{~g}$ supernatant fraction of rabbit lung homogenates of several aromatic amines (2-aminoanthracene, 2-aminofluorene, 2-acetylaminofluorene) and aflatoxin $\mathrm{B}_{1}$ to metabolites mutagenic in the Ames test. The metabolic activation by the rabbit lung fraction was more effective than that by the corresponding rabbit liver fraction. Also more revertant colonies were obtained per nanomol of total CYP by the lung preparations compared with those obtained from the liver. They then showed that purified CYP4B1, but not CYP2B4 was responsible for their metabolic activation.

2-Aminofluorene was recognized as a lead substrate of CYP4B1 (Vanderslice et al. 1987).

Slaughter et al. (1983) demonstrated the importance of pulmonary CYP4B1 catalytic activity for the metabolic activation of the lung toxin 4-ipomeanol by observing inhibition of its covalent binding to rabbit lung microsomes by antiCYP4B1 antibodies. Czerwinski et al. (1991) demonstrated highly efficient activation of 4-ipomeanol by recombinant rabbit CYP4B1 (leading under the conditions of the study to covalent binding to cellular DNA of $84.23 \pm 15.85 \mathrm{dpm} / \mu \mathrm{g}$ DNA compared with recombinant human CYP4B1 leading to $0.98 \pm 0.18 \mathrm{dpm} / \mu \mathrm{g} \mathrm{DNA}$ ).

Thornton-Manning et al. (1996) showed that recombinant rabbit CYP4B1 efficiently activated the lung toxin 3-methylindole to the reactive 3-methylindoleimine in amounts 
exceeding those produced by the human CYP most active in this respect, CYP2F1.

\section{Non-CYP oxidoreductases}

\section{Flavin-dependent monooxygenases (FMO)}

Overby et al. (1992) reported that in the rabbit lung Clara (Club) cells of the terminal bronchiole were the major location of FMO. Much lower FMO levels have been found in ciliated cells throughout the airways, alveolar type I and type II cells, endothelial cells as well as in the lining layer of trachea.

\section{Aldo-keto reductases (AKR)}

Endo et al. (2014) showed that of several AKR tested AKR1C32 transcripts were especially highly expressed in rabbit lung. Recombinant rabbit AKR1C32 metabolized naloxone, metyrapone, loxoprofen as well as 3-, 17- and 20-ketosteroids.

\section{Hydrolases}

\section{Epoxide hydrolase (EH)}

Microsomal epoxide hydrolase ( $\mathrm{mEH}$, also called EPHX1) Devereux et al. (1985) reported the observation of $\mathrm{mEH}$ activity toward BP 4,5-epoxide as substrate in rabbit lung, much (about tenfold) higher in Clara (Club) cells as compared with type II pneumocytes.

Soluble epoxide hydrolase (sEH, also called EPHX2) sEH was observed in the rabbit lung where its specific activity was - after liver and kidney - third highest of seven investigated rabbit organs ( $1 / 10$ of the specific activity in the liver) (Gill and Hammock 1980).

\section{Xenobiotica-metabolizing enzymes in the hamster lung}

\section{Cytochromes P450 (CYP)}

\section{CYP catalytic activities (Table 3)}

Lorenz et al. (1984) showed that hamster lung microsomes efficiently catalyzed the deethylation of the broad-spectrum CYP substrate 7-ethoxycoumarin, about 5-fold slower than mouse lung microsomes, similar as rat lung microsomes, but more than 100-fold faster than human lung microsomes.

Hamster lung Clara (Club) cells were very early on identified as a prime location for CYP-dependent covalent binding of 4-ipomeanol, a pneumotoxin in rodents and cattle, these Clara cells being associated with 4-ipomeanol selective toxicity (Boyd 1977).

Fong and Rasmussen (1987) observed 6-O-ethylguanine produced by the treatment of hamsters with $\mathrm{N}$-nitrosodiethylamine to occur in type II pneumocytes and alveolar macrophages, but much more in Clara cells.

Paolini et al. (1995) reported the presence of CYP2Bdependent PROD activity in the Syrian hamster lung and its inducibility by several compounds (sodium phenobarbital, sodium barbital, phorone, cyclophosphamide).

Hoet et al. (1997) showed in hamster lung slices EROD activity, which was increased upon pretreatment of the slices with 3-methylcholanthrene or BNF. BROD activity was decreased after pretreatment with 3-methylcholanthrene, but increased after pretreatment with BNF.

\section{Conjugating enzymes}

\section{Glutathione S-transferase (GST)}

Autrup et al. (1980) showed in cultured hamster tracheobrochial tissue a high proportion of BP metabolized to glutathione metabolites, much more than to glucuronides.

\section{Sulfotransferase (SULT)}

Autrup et al. (1980) showed in cultured hamster tracheobrochial tissue a high proportion of BP metabolized to sulfates, much more than to glucuronides.

\section{Xenobiotica-metabolizing enzymes in the guinea pig lung \\ Cytochromes P450 (CYP)}

Bilimoria et al. (1977) observed in guinea pig lung total homogenate AHH activity (see Table 3), which was substantially (by about 50\%) reduced after exposure of the animals to cigarette smoke (whole smoke from nicotinefree cigarettes). Benzo[a]pyrene applied i.p. induced guinea pig lung AHH (about 1.7-fold), while 3-methylcholanthrene or Aroclor 1254 did not. 


\section{Xenobiotica-metabolizing enzymes in the pig lung}

\section{Cytochromes P450 (CYP)}

\section{CYP transcript expression}

CYP1A1 and CYP1A2 transcripts have been observed in the pig lung (Chirulli et al. 2007; Messina et al. 2008). In the former study a considerable increase of CYP1A1 mRNA levels upon pretreatment of the pigs with BNF has been observed, and a minimal increase of CYP1A2 as well. CYP1B1 mRNA was observed only after pretreatment with BNF.

Pig CYP2B22 (homologous to human CYP2B6, 81\% nucleotide identity) mRNA was constitutively expressed in pig lung at higher levels than in the liver (Nannelli et al. 2008).

CYP2D25 (homologous to human CYP2D6, 83\% nucleotide identity) transcripts have been observed-at low levels_-in pig lung (Hosseinpour and Wikvall 2000).

CYP2E1 transcript expression in the minipig lung was reported by Köhler et al. (2006a, b).

Pig CYP3A22, 3A29 and 3A46 mRNA were present in the pig lung, but at much lower levels than in the liver. They were increased upon pretreatment of the pigs with rifampicin (Nannelli et al. 2008). CYP3A22, CYP3A29 and CYP3A46 transcripts were also observed in the pig lung by Messina et al. (2009).

\section{CYP and related proteins expression}

Dees et al. (1980) observed NADPH CYP reductase protein in the bronchial and bronchiolar epithelia and alveolar wall of minipig lung, indistinguishable between phenobarbital-treated and control rats.

Chirulli et al. (2007) observed in the pig lung protein bands immunoreactive with anti-rat CYP1A1 and with anti-rat CYP1B1. Both of them were increased upon treatment of the pigs with BNF. The same team (Nannelli et al. 2008) reported the presence of CYP2B and CYP3A protein in the pig lung. They were not increased by pretreatment of the pigs with rifamicin.

Roos et al. (2002) observed in the minipig lung-in contrast to liver, kidney and duodenum-CYP1A1 protein, albeit at low levels, which were more than 10 -fold increased upon oral administration of PAH-contaminated soil.

CYP2E1 protein expression in the minipig lung was reported by Köhler et al. (2006b).

\section{CYP catalytic activities (Table 3)}

Chirulli et al. (2007) observed in the pig lung EROD (prototypic for CYP1A1), MROD (prototypic for CYP1A2), $17 \beta$-estradiol 4-hydroxylation (prototypic for CYP1B1) as well as PROD and EFCOD (7-ethoxy-4-trifluoromethylcoumarin deethylase) (both of them prototypic for CYP2B) activities. EROD, MROD and PROD activities were increased upon pretreatment of the pigs with BNF, acetanilide 4-hydroxylation was detected only after pretreatment with BNF. In subsequent work (Nannelli et al. 2008) they showed in pig lung BQOD (7-benzyloxyquinoline $O$-debenzylase), EFCOD, BROD and 9-anthraldehyde oxidase activities. None of these activities was increased upon pretreatment of the pigs with rifampicin.

Köhler et al. (2006b) observed in the minipig lung hydroxylation of the CYP2E1 prototypic substrate chlorzoxazone.

\section{Conjugating enzymes}

\section{Glutathione S-transferase (GST)}

Pettigrew et al. (2001) showed that pig lung GSTP is selectively labelled and inactivated by 3-methyleneoxindole. Striking protection against the inactivation by $S$-(hydroxyethyl)ethacrynic acid indicated that 3-methylenoxindole bound to the active site involving both, the xenobiotic substrate binding site and the glutathione binding site. Labelling with radioactive 3-methyleneoxindole showed its binding to Trp38. The authors state that this information may be useful for developing optimized combination chemotherapy since GSTP is selectively overexpressed in many tumors.

\section{Xenobiotica-metabolizing enzymes in the dog lung}

\section{Cytochromes P450 (CYP)}

\section{CYP transcript expression}

Visser et al. (2017) observed in dog lung the expression of CYP2B11 transcripts and minimal expression of other xenobiotic metabolizing enzymes mRNAs.

\section{CYP catalytic activities (Table 1)}

CYP-catalyzed monooxygenation of several xenobiotics has been observed in the dog lung (Bond et al. 1988) (see Table 3). Specific activities for BP, 1-nitropyrene and 7-ethoxycoumarin as substrates were considerably higher 
in bronchi, bronchioles and peripheral lung compared with trachea and larynx, for BP and 7-ethoxycoumarin highest in bronchioli (Bond 1993). Activities were considerably lower than in the dog liver. They also were considerably lower than those for EH, GST and UGT in the dog lung with respect to the substrates used for these assays (compare Tables 3, 5,6 and 7).

\section{Hydrolases}

\section{Epoxide hydrolase (EH) (Table 5)}

EH activity was observed in the dog lung by Bond et al. (1988). The authors used the assay developed by Oesch et al. (1971). They did not separate the soluble from the microsomal fraction of the cell homogenate. It, therefore, cannot be concluded whether the determined activity is due to $\mathrm{mEH}$ or $\mathrm{sEH}$ or both, since styrene 7,8-oxide, which is used in this assay, is a substrate of both, $\mathrm{mEH}$ and sEH. Activities were surprisingly high, similar to the activities in the dog liver. The activities were considerably (about 3 - to 4-fold) higher in the 10-18-generation bronchial ramifications (distal airways) compared with the mainstem bronchi and compared with the peripheral lung.

\section{Conjugating enzymes}

\section{Glutathione S-transferase (GST)}

Bond et al. (1988) observed considerable GST activities for styrene 7,8-oxide as substrate in the dog lung (Table 6). Activities were in the same order of magnitude (about one third) as those in the dog liver.

\section{UDP-glucuronosyltransferase (UGT) (Table 7)}

UGT activities were observed in the dog lung by Bond et al. (1988) using umbelliferone as substrate. Activities were relatively high, about half as high as those observed in the dog liver. However, these UGT activities in the dog lung were considerably lower than the EH and GST activities in the dog lung (about 6- to 60-fold lower with respect to the substrates used for the assays).

\section{Xenobiotica-metabolizing enzymes in the cat lung}

\section{Cytochromes P450 (CYP)}

Okamatsu et al. (2017) observed in cat lung CYP2B6 transcripts, protein and activity. Of eight organs investigated transcripts were by far most abundant in lung, modest in small intestine, low in colon and rectum, very low in brain and heart and practically nil in liver and kidney. Western blot analysis showed the presence of CYP2B6 protein in cat lung, but not in liver. Heterologously expressed cat $2 \mathrm{~B} 6$ protein metabolized several typical human CYP2B6 substrates (see Table 3) such as 7-ethoxy-4-(trifluoromethyl)-coumarin.

\section{Xenobiotica-metabolizing enzymes in the goat lung}

Xenobiotic-metabolizing enzymes in the goat lung are of special interest because of the selective toxicity of several compounds to the goat lung.

\section{Cytochromes P450 (CYP)}

\section{CYP transcript expression}

The presence of CYP4B transcripts in goat lung was demonstrated by Ramakanth et al. (1994).

\section{CYP catalytic activities (Table 3)}

Early studies showed that the goat pneumotoxin 3-methylindole was toxified by goat lung CYP, in that the covalent binding of 3-methylindole to goat lung microsomal protein was inhibited by SKF 525A (Becker et al. 1984) and by the CYP suicide substrate 1-aminobenzotriazole (Huijzer et al. 1989). Wang et al. (1998) confirmed the metabolism of 3 -methylindole in the goat lung. The goat form of the lungselective CYP2F subfamily, 2F3, which has $82 \%$ homology with the human and with the mouse forms, accounted for $20 \%$ of the total 3-methylindole metabolism in the goat lung. CYP4B2 was cloned from goat lung and used for recombinant production of CYP4B2 which toxified 3-methylindole to its reactive metabolite 3-methyleneindolenine (Carr et al. 2003b).

Goat recombinant CYP2F3 catalyzed the biotransformation of 1,1-dichloroethylene to glutathionyl acetate putatively generated via dichloroethylene epoxide (Simmonds et al. 2004).

\section{Xenobiotica-metabolizing enzymes in the duck lung}

\section{Flavin-dependent monooxygenase (FMO)}

The Flavin-dependent monooxygenase FMO3 is the major FMO in the human liver. It is important in the metabolism of many xenobiotic compounds, but also for metabolically neutralizing the extremely bad odor of trimethylamine which is 
not only a serious problem for human individuals deficient in this enzyme, but also for bad odor of nutritional components including duck meat. Wang et al. (2013) have therefore cloned and characterized the Pekin duck FMO3 cDNA. They reported that duck FMO3 transcripts were dramatically expressed in the duck lung (as well as liver and kidney, very much more than in 12 other tissues).

\section{Xenobiotica-metabolizing enzymes in the monkey lung}

\section{Marmoset}

\section{Cytochromes P450 (CYP)}

Uehara et al. (2018) demonstrated in marmoset lung high expression of CYP2F1 transcripts, much higher than in any of the other investigated organs (liver, kidney, jejunum, brain). By immunoblotting they found in marmoset lung CYP2F1 protein cross-reactive with human CYP2F1 antibodies. In marmoset lung microsomes they observed catalytic activity for several prototype CYP substrates, including coumarin, 7-ethoxycoumarin, 2-/4-biphenyl, but not chlorzoxazone. Table 3 (values with superscript/footnote $\mathrm{x}$ ) shows that the rates of these reactions are highly similar to/almost identical as those of the human lung.

\section{Cynomolgus}

\section{Cytochromes P450 (CYP)}

Uehara et al. (2018) demonstrated in cynomolgus monkey lung high expression of CYP2F1 transcripts, of ten investigated organs third highest after testis and ovaries. In cynomolgus monkey lung microsomes they observed catalytic activity for several prototypic CYP substrates, including coumarin, 7-ethoxycoumarin, 2-/4-biphenyl, but not chlorzoxazone (Table 3).

\section{Rhesus}

\section{Flavin-dependent monooxygenase (FMO)}

Krueger et al. (2001) demonstrated that in the Rhesus monkey's predominant lung FMO, FMO2, a relatively small sequence of the C-terminal portion ( $\mathrm{mFMO} 2471-535)$ is essential for stability and enzymatic activity.

\section{Baboon}

\section{Cytochromes P450 (CYP)}

In an early study Wheeler et al. (1990) characterized in human and baboon lung a CYP homologous to the toxicologically important CYP1A1 of rodents. Antibodies to rat CYP1A1 cross reacted with a human and a baboon lung microsomal protein with similar affinities between these two species, but these proteins of both species bound to a considerably lesser amount than the corresponding rat and rabbit lung mirosomal proteins did. Typical CYP1A enzymatic activities were found in human and baboon lung microsomes, in similar activities between these two species, but considerably lower activities as compared with rat and rabbit microsomoses (see Table 3 comparing the Wheeler et al. 1990 data between these species).

\section{Xenobiotica-metabolizing enzymes in the human lung}

\section{Cytochromes P450 (CYP)}

\section{CYP and CYP reductase transcript expression}

Zuo et al. (2018) demonstrated in samples - gained by bronchoscopy and by scraping from the human small airways epithelial Clara (Club) cells - transcripts of POR (cytochrome P450 oxidoreductase).

Reports on individual CYPs transcripts expression in the human lung are partly conflicting. For a quick overview on the transcript expression of the three major xenobioticametabolizing CYP families CYP1, CYP2 and CYP3 see Table 8 (conflicting results are indicated by "+/-").

The major CYPs expressed in the human liver have also been found to be expressed in the human lung, but some distinct preferences are obvious. Contrasts between the transcript expression in the lung versus liver are given in bold letters/symbols in Table 8 . Preferential or specific constitutive expression in the lung was observed for CYP1B1, 2F1, $2 \mathrm{~S} 1$ and 4B1 (the latter exclusively in the lung). In addition the following CYP mRNAs have consistently been reported to be present in the human lung: 2A6, 2A13, 2B6/7, 2E1, 2J2, 2R1, 3A5.

Details follow here:

Human CYP1A1 transcripts were found in the human lung by Omiecinski et al. (1990) and McLemore et al. (1990), in the latter studies only in smokers but not in those who had quit smoking since more than 6 weeks. Female smokers had considerably (more than 2-fold) higher expression of CYP1A1 mRNA than had male smokers and the amount of hydrophobic DNA adducts 
Table 8 Expression levels of genes involved in xenobiotic metabolism in 10 lung cell lines, primary cultures of HBEC, and lung tissues. Adapted from Courcot et al. (2012), with permission of the publisher

\begin{tabular}{|c|c|c|c|c|c|c|c|c|c|c|c|c|c|c|c|}
\hline \multirow[t]{2}{*}{ Gene } & \multicolumn{11}{|c|}{ Lung cell models } & \multicolumn{4}{|c|}{ Lung tissues } \\
\hline & $16 \mathrm{HBE}$ & 1HAE0 & A549 & BEAS-2B & Calu-1 & L-132 & $\mathrm{H} 292$ & H358 & $\mathrm{H} 460$ & $\mathrm{H} 727$ & HBEC & $\mathrm{BM}$ & $\mathrm{PP}$ & $\mathrm{SCC}$ & $\mathrm{AC}$ \\
\hline \multicolumn{16}{|c|}{ Phase I enzymes } \\
\hline$A A D A C$ & - & - & +++ & - & + & - & & - & + & - & - & + & ++ & + & + \\
\hline$A B P 1$ & - & - & - & - & - & - & + & + & - & +++ & - & + & + & + & ++ \\
\hline$A D H 1 A^{*}$ & - & - & - & - & - & - & - & - & - & - & - & - & - & - & - \\
\hline$A D H 1 B$ & - & - & - & ++ & - & - & - & - & - & - & - & +++ & +++ & ++ & ++ \\
\hline$A D H 1 C$ & - & - & - & & - & - & - & + & - & - & - & ++ & + & + & + \\
\hline$A D H 4$ & - & - & - & - & - & - & - & - & - & - & - & + & - & - & - \\
\hline$A D H 5$ & ++ & ++ & ++ & ++ & ++ & + & ++ & ++ & ++ & ++ & ++ & ++ & ++ & ++ & ++ \\
\hline ADH6 & - & - & - & - & - & - & - & - & - & - & - & ++ & - & - & - \\
\hline$A D H 7$ & - & - & - & - & - & - & - & - & - & - & ++ & +++ & - & ++ & - \\
\hline ADHFE1 & + & + & + & ++ & + & - & + & + & + & + & + & ++ & ++ & ++ & ++ \\
\hline$A K R 1 A 1$ & +++ & +++ & +++ & +++ & +++ & ++ & +++ & ++ & +++ & ++ & +++ & +++ & +++ & +++ & +++ \\
\hline$A K R 1 B 1$ & +++ & +++ & +++ & +++ & +++ & +++ & +++ & +++ & +++ & +++ & +++ & +++ & +++ & +++ & +++ \\
\hline$A K R 1 B 10$ & - & + & +++ & + & + & - & + & + & +++ & + & ++ & ++ & - & +++ & + \\
\hline$A K R 1 C 1 / 2$ & - & - & +++ & + & ++ & ++ & + & & +++ & + & +++ & ++ & ++ & +++ & ++ \\
\hline$A K R 1 C 3$ & - & - & +++ & ++ & ++ & ++ & + & + & +++ & ++ & ++ & ++ & ++ & +++ & ++ \\
\hline$A K R 1 C 4$ & - & - & - & - & - & - & - & - & - & $\ldots$ & - & - & - & - & - \\
\hline$A K R I C L 1$ & - & - & + & - & - & - & - & - & - & - & - & + & - & + & + \\
\hline AKR1CL2 & ++ & - & +++ & ++ & ++ & - & ++ & ++ & + & - & + & ++ & ++ & ++ & ++ \\
\hline$A K R 1 D 1$ & - & - & - & - & - & - & $\ldots$ & $\ldots$ & - & - & - & - & - & - & - \\
\hline AKR6A3 & - & - & - & - & - & - & - & - & - & - & - & + & ++ & + & + \\
\hline$A K R 6 A 5$ & ++ & ++ & +++ & ++ & ++ & ++ & ++ & ++ & ++ & + & + & ++ & +++ & ++ & ++ \\
\hline AKR6A9 & - & - & - & + & + & - & + & $\ldots$ & - & - & - & + & + & + & + \\
\hline$A K R 7 A 2$ & +++ & +++ & +++ & ++ & ++ & + & ++ & ++ & ++ & ++ & ++ & ++ & ++ & ++ & ++ \\
\hline$A K R 7 A 3$ & ++ & + & + & + & ++ & $\ldots$ & + & + & + & +++ & + & ++ & + & + & ++ \\
\hline$A K R 7 L$ & - & - & - & - & - & - & - & - & - & $\ldots$ & - & - & - & - & - \\
\hline$A L D H 16 A 1$ & + & + & + & + & + & $\ldots$ & + & + & + & - & + & + & + & + & + \\
\hline$A L D H 18 A 1$ & +++ & +++ & +++ & +++ & +++ & ++ & +++ & ++ & +++ & ++ & +++ & +++ & ++ & +++ & +++ \\
\hline$A L D H 1 A 1$ & - & - & +++ & - & + & - & $\ldots$ & - & +++ & ++ & ++ & +++ & +++ & ++ & ++ \\
\hline$A L D H 1 A 2$ & - & + & - & - & - & - & + & - & - & + & - & ++ & ++ & + & + \\
\hline$A L D H I A 3$ & +++ & +++ & +++ & +++ & +++ & ++ & +++ & +++ & + & + & +++ & +++ & ++ & ++ & ++ \\
\hline$A L D H 1 B 1$ & +++ & ++ & +++ & ++ & ++ & ++ & ++ & ++ & ++ & + & ++ & ++ & ++ & ++ & ++ \\
\hline$A L D H 1 L 1$ & ++ & + & + & ++ & - & - & ++ & + & + & - & + & ++ & + & + & + \\
\hline$A L D H 2$ & - & ++ & +++ & +++ & ++ & ++ & +++ & + & ++ & ++ & +++ & +++ & +++ & +++ & +++ \\
\hline$A L D H 3 A 1$ & + & - & +++ & +++ & ++ & + & ++ & + & +++ & - & ++ & +++ & + & ++ & + \\
\hline$A L D H 3 A 2$ & +++ & ++ & +++ & +++ & +++ & ++ & +++ & +++ & +++ & ++ & +++ & +++ & +++ & +++ & +++ \\
\hline$A L D H 3 B 1$ & +++ & ++ & +++ & +++ & +++ & ++ & +++ & ++ & +++ & + & ++ & +++ & +++ & ++ & +++ \\
\hline$A L D H 3 B 2$ & - & + & - & - & - & - & + & $\ldots$ & - & - & ++ & + & - & + & - \\
\hline$A L D H 4 A 1$ & +++ & +++ & +++ & +++ & +++ & + & ++ & ++ & ++ & + & ++ & ++ & ++ & ++ & ++ \\
\hline$A L D H 5 A 1$ & ++ & ++ & ++ & ++ & + & + & ++ & ++ & ++ & + & + & ++ & ++ & ++ & ++ \\
\hline$A L D H 6 A 1$ & ++ & +++ & ++ & +++ & +++ & + & ++ & ++ & ++ & ++ & ++ & ++ & ++ & ++ & ++ \\
\hline$A L D H 7 A 1$ & +++ & +++ & +++ & +++ & +++ & ++ & +++ & ++ & ++ & ++ & +++ & ++ & ++ & ++ & ++ \\
\hline$A L D H 8 A 1$ & - & - & - & - & - & - & - & $\ldots$ & - & - & - & + & + & + & + \\
\hline$A L D H 9 A 1$ & +++ & ++ & +++ & +++ & +++ & ++ & +++ & +++ & +++ & ++ & ++ & +++ & +++ & ++ & +++ \\
\hline$A O C 2$ & + & ++ & ++ & - & + & + & - & + & + & + & + & + & + & + & + \\
\hline$A O C 3$ & - & + & + & - & + & $\ldots$ & $\ldots$ & + & + & $\ldots$ & + & ++ & +++ & ++ & ++ \\
\hline$A O F 1$ & +++ & ++ & +++ & ++ & +++ & ++ & ++ & ++ & ++ & + & ++ & +++ & ++ & ++ & ++ \\
\hline$A O F 2$ & +++ & +++ & +++ & +++ & +++ & ++ & ++ & ++ & ++ & ++ & ++ & ++ & ++ & ++ & ++ \\
\hline
\end{tabular}


Table 8 (continued)

\begin{tabular}{|c|c|c|c|c|c|c|c|c|c|c|c|c|c|c|c|}
\hline \multirow[t]{2}{*}{ Gene } & \multicolumn{11}{|c|}{ Lung cell models } & \multicolumn{4}{|c|}{ Lung tissues } \\
\hline & $16 \mathrm{HBE}$ & 1HAE0 & A549 & BEAS-2B & Calu-1 & L-132 & H 292 & H358 & $\mathrm{H} 460$ & $\mathrm{H} 727$ & HBEC & $\mathrm{BM}$ & $\mathrm{PP}$ & $\mathrm{SCC}$ & $\mathrm{AC}$ \\
\hline$A O X 1$ & ++ & + & ++ & ++ & +++ & - & + & + & ++ & + & ++ & ++ & ++ & + & ++ \\
\hline BCHE & - & - & + & - & - & - & - & - & ++ & + & - & ++ & ++ & + & + \\
\hline$C B R 1$ & +++ & +++ & +++ & +++ & ++ & ++ & ++ & ++ & ++ & + & ++ & ++ & ++ & +++ & ++ \\
\hline$C B R 3$ & ++ & + & ++ & ++ & ++ & + & $\ldots$ & + & + & - & + & ++ & + & ++ & + \\
\hline$C B R 4$ & ++ & ++ & ++ & ++ & ++ & + & ++ & ++ & ++ & + & ++ & ++ & ++ & ++ & ++ \\
\hline CES1 & - & - & +++ & - & - & - & - & - & ++ & + & + & +++ & +++ & +++ & +++ \\
\hline CES2 & ++ & ++ & ++ & ++ & ++ & + & ++ & ++ & ++ & + & +++ & ++ & ++ & ++ & ++ \\
\hline CES3 & ++ & ++ & ++ & + & + & + & + & + & + & $\ldots$ & + & ++ & ++ & + & ++ \\
\hline CES4 & - & - & - & - & - & - & - & - & - & - & - & + & + & + & - \\
\hline CES7* & - & - & - & - & - & - & - & - & - & - & - & - & - & - & - \\
\hline CYP1A1 & - & ++ & + & $\ldots$ & + & + & + & + & - & + & ++ & - & - & - & - \\
\hline CYP1A2* & - & - & - & - & - & - & - & - & - & - & - & - & - & - & - \\
\hline$C Y P 1 B 1$ & +++ & ++ & +++ & +++ & +++ & +++ & ++ & ++ & +++ & ++ & ++ & ++ & +++ & ++ & +++ \\
\hline СYР2A6 & - & - & - & - & - & - & $\ldots$ & - & - & - & - & - & - & - & - \\
\hline$C Y P 2 A 7$ & - & - & + & $\ldots$ & + & - & + & $\ldots$ & - & - & - & ++ & ++ & + & + \\
\hline CYP $2 A 13$ & - & - & - & - & - & - & + & - & - & - & - & ++ & - & - & - \\
\hline СYР2B6 & - & - & - & - & $\ldots$ & - & + & - & - & + & - & ++ & ++ & + & ++ \\
\hline СYР $2 C 8$ & - & - & - & - & - & - & - & - & - & - & - & + & - & - & - \\
\hline CYP2C9 & - & - & + & - & - & - & - & - & - & - & + & ++ & + & - & + \\
\hline СYР2C18 & - & - & + & - & - & - & - & - & - & - & ++ & ++ & + & + & + \\
\hline СYР $2 C 19$ & - & - & - & - & - & - & - & - & - & - & - & + & + & + & + \\
\hline CYP2D6 & - & - & - & - & - & - & + & - & - & - & - & + & - & + & + \\
\hline$C Y P 2 E 1$ & + & + & - & - & + & - & + & + & + & + & ++ & ++ & ++ & ++ & ++ \\
\hline$C Y P 2 F 1$ & - & - & - & - & - & - & - & - & - & - & - & +++ & + & - & - \\
\hline$C Y P 2 J 2$ & ++ & ++ & + & - & + & - & ++ & ++ & $\ldots$ & - & ++ & ++ & + & + & ++ \\
\hline$C Y P 2 R 1$ & - & ++ & ++ & + & ++ & + & ++ & ++ & ++ & ++ & ++ & ++ & ++ & ++ & ++ \\
\hline$C Y P 2 S 1$ & ++ & ++ & +++ & ++ & ++ & + & ++ & ++ & + & + & ++ & ++ & ++ & +++ & ++ \\
\hline$C Y P 2 U 1$ & ++ & ++ & ++ & ++ & +++ & + & ++ & ++ & ++ & + & ++ & ++ & ++ & ++ & ++ \\
\hline$C Y P 2 W 1$ & - & - & - & - & + & - & + & - & - & - & + & ++ & - & + & - \\
\hline CYP $3 A 4^{*}$ & - & - & - & - & - & - & - & - & - & - & - & - & - & - & - \\
\hline СYР3А5 & - & - & ++ & - & - & - & + & - & - & ++ & + & + & ++ & - & + \\
\hline СYР3A7 & - & - & - & - & - & - & - & - & - & - & - & - & + & - & - \\
\hline СYР $3 A 43 *$ & - & - & - & - & - & - & - & - & - & - & - & - & - & - & - \\
\hline CYP4A11/22 & - & - & - & + & - & - & - & - & - & - & \pm & - & - & - & - \\
\hline$C Y P 4 B 1$ & - & - & - & ++ & - & - & + & - & - & - & ++ & +++ & +++ & + & ++ \\
\hline$C Y P 4 F 2 *$ & - & - & - & - & - & - & - & - & - & - & - & - & - & - & - \\
\hline$C Y P 4 F 3$ & + & - & + & - & - & - & $\ldots$ & - & - & - & - & + & ++ & + & + \\
\hline CYP4F8* & - & - & - & - & - & - & - & - & - & - & - & - & - & - & - \\
\hline$C Y P 4 F 11$ & - & ++ & +++ & ++ & + & + & ++ & - & +++ & + & ++ & ++ & + & ++ & + \\
\hline CYP4F12 & - & + & ++ & + & + & - & + & - & - & + & + & ++ & ++ & + & + \\
\hline CYP4F22 & - & + & - & - & - & - & - & - & - & $\ldots$ & + & + & + & + & + \\
\hline CYP4V2 & ++ & + & ++ & ++ & ++ & + & ++ & ++ & ++ & + & ++ & ++ & ++ & ++ & ++ \\
\hline CYP $4 X 1$ & - & + & - & ++ & - & - & + & + & + & - & + & +++ & ++ & ++ & ++ \\
\hline CYP4Z1 & - & - & - & + & - & - & - & - & - & - & - & ++ & + & - & + \\
\hline CYP5A11 & - & + & +++ & + & ++ & + & - & + & + & + & + & ++ & ++ & ++ & ++ \\
\hline CYP7A1* & - & - & - & - & - & - & - & - & - & - & - & - & - & - & - \\
\hline$C Y P 7 B 1$ & ++ & - & ++ & + & - & - & + & + & - & - & + & ++ & ++ & ++ & ++ \\
\hline СYР8А1 & ++ & - & - & ++ & ++ & - & + & - & - & - & - & ++ & +++ & ++ & ++ \\
\hline
\end{tabular}


Table 8 (continued)

\begin{tabular}{|c|c|c|c|c|c|c|c|c|c|c|c|c|c|c|c|}
\hline \multirow[t]{2}{*}{ Gene } & \multicolumn{11}{|c|}{ Lung cell models } & \multicolumn{4}{|c|}{ Lung tissues } \\
\hline & $16 \mathrm{HBE}$ & 1HAE0 & A549 & BEAS-2B & Calu-1 & L-132 & $\mathrm{H} 292$ & H358 & $\mathrm{H} 460$ & $\mathrm{H} 727$ & HBEC & $\mathrm{BM}$ & $\mathrm{PP}$ & SCC & $\mathrm{AC}$ \\
\hline CYP8B1 & - & - & - & - & - & - & - & - & - & - & - & + & + & + & + \\
\hline$C Y P 11 B 1^{*}$ & - & - & - & - & - & - & - & - & - & - & - & - & - & - & - \\
\hline$C Y P 11 B 2 *$ & - & - & - & - & - & - & - & - & - & - & - & - & - & - & - \\
\hline CYP17A1 & - & - & - & - & - & - & - & - & - & - & - & - & + & - & + \\
\hline CYP19A1 & - & - & - & - & $\ldots$ & - & - & $\ldots$ & - & - & - & - & - & + & - \\
\hline CYP20A1 & +++ & ++ & ++ & ++ & ++ & + & ++ & ++ & ++ & ++ & ++ & ++ & ++ & ++ & ++ \\
\hline$C Y P 21 A 2$ & - & $\ldots$ & - & + & $\ldots$ & - & + & - & - & - & - & + & + & - & + \\
\hline$C Y P 24 A 1$ & +++ & ++ & +++ & - & +++ & - & ++ & + & - & - & ++ & ++ & - & ++ & ++ \\
\hline CYP26A1 & - & + & ++ & - & - & - & + & + & - & - & - & ++ & - & ++ & + \\
\hline$C Y P 26 B 1$ & + & + & +++ & - & ++ & - & + & + & - & - & + & ++ & ++ & ++ & ++ \\
\hline$C Y P 26 C 1 *$ & - & - & - & - & - & - & - & - & - & - & - & - & - & - & - \\
\hline$C Y P 27 A 1$ & - & - & - & ++ & ++ & - & - & - & - & - & + & ++ & +++ & ++ & ++ \\
\hline$C Y P 27 B 1$ & ++ & + & ++ & ++ & ++ & $\ldots$ & ++ & ++ & ++ & ++ & ++ & + & ++ & ++ & ++ \\
\hline$C Y P 27 C 1$ & +++ & ++ & ++ & + & + & $\ldots$ & - & ++ & - & - & ++ & + & - & + & + \\
\hline СYР39A1 & + & - & + & ++ & + & $\ldots$ & + & + & + & + & + & ++ & ++ & + & + \\
\hline CYP $46 A 1$ & - & - & - & - & $\ldots$ & - & - & - & - & - & - & - & - & - & - \\
\hline CYP51A1 & +++ & +++ & +++ & +++ & +++ & ++ & ++ & ++ & ++ & ++ & +++ & ++ & ++ & ++ & ++ \\
\hline DHRS2 & +++ & ++ & ++ & + & ++ & $\ldots$ & ++ & +++ & + & + & - & - & - & + & + \\
\hline DHRS4 & +++ & +++ & +++ & ++ & +++ & ++ & ++ & ++ & ++ & ++ & ++ & ++ & ++ & ++ & ++ \\
\hline DHRS9 & - & - & ++ & - & $\ldots$ & - & + & $\ldots$ & - & + & ++ & +++ & ++ & ++ & ++ \\
\hline$D P Y D$ & +++ & ++ & ++ & ++ & +++ & + & ++ & ++ & ++ & - & + & +++ & +++ & ++ & +++ \\
\hline EPHXI & +++ & ++ & +++ & +++ & +++ & ++ & ++ & ++ & +++ & ++ & ++ & +++ & +++ & +++ & +++ \\
\hline EPHX2 & ++ & ++ & +++ & ++ & + & + & ++ & ++ & ++ & ++ & ++ & ++ & ++ & ++ & ++ \\
\hline$E S D$ & +++ & +++ & +++ & +++ & +++ & ++ & +++ & ++ & ++ & ++ & ++ & ++ & ++ & ++ & ++ \\
\hline FMOI & - & - & - & - & - & - & - & - & - & - & - & + & + & ++ & ++ \\
\hline FMO2 & - & - & - & + & - & - & - & - & - & - & - & +++ & +++ & ++ & ++ \\
\hline$F M O 3$ & - & - & - & + & - & - & - & - & - & - & \pm & ++ & ++ & ++ & ++ \\
\hline FMO4 & + & + & + & + & + & - & + & + & + & $\ldots$ & + & ++ & ++ & ++ & + \\
\hline FMO5 & + & + & ++ & + & + & + & + & ++ & + & ++ & + & +++ & +++ & ++ & ++ \\
\hline HSD $17 B 10$ & +++ & +++ & +++ & +++ & +++ & ++ & +++ & +++ & +++ & ++ & +++ & +++ & +++ & +++ & +++ \\
\hline$M A O A$ & ++ & ++ & ++ & +++ & +++ & + & +++ & ++ & ++ & ++ & +++ & +++ & +++ & +++ & +++ \\
\hline$M A O B$ & - & + & ++ & ++ & + & + & $\ldots$ & + & - & - & + & ++ & +++ & ++ & ++ \\
\hline$N Q O 1$ & +++ & +++ & +++ & +++ & +++ & +++ & +++ & +++ & +++ & +++ & +++ & +++ & ++ & +++ & +++ \\
\hline$N Q O 2$ & +++ & ++ & +++ & ++ & +++ & ++ & ++ & ++ & +++ & ++ & ++ & ++ & ++ & ++ & ++ \\
\hline$P A O X$ & ++ & + & ++ & ++ & ++ & - & ++ & + & + & + & ++ & ++ & + & ++ & ++ \\
\hline PON1 & - & - & + & - & - & - & - & - & - & - & - & - & + & - & + \\
\hline PON2 & +++ & ++ & +++ & +++ & +++ & ++ & +++ & ++ & ++ & ++ & +++ & ++ & +++ & +++ & +++ \\
\hline PON3 & ++ & + & +++ & + & + & - & ++ & + & + & + & + & + & ++ & + & ++ \\
\hline$S P R$ & +++ & ++ & +++ & ++ & ++ & ++ & ++ & ++ & ++ & ++ & ++ & ++ & ++ & ++ & ++ \\
\hline SUOX & ++ & ++ & ++ & ++ & ++ & + & ++ & ++ & ++ & + & ++ & ++ & ++ & ++ & ++ \\
\hline$X D H$ & ++ & + & ++ & + & + & - & ++ & + & - & - & ++ & ++ & + & + & ++ \\
\hline \multicolumn{16}{|c|}{ Phase II enzymes } \\
\hline$A A N A T^{*}$ & - & - & - & - & - & - & - & - & - & - & - & - & - & - & - \\
\hline$A S 3 M T$ & ++ & ++ & ++ & ++ & ++ & - & ++ & + & ++ & + & + & ++ & ++ & ++ & ++ \\
\hline$B A A T$ & - & - & ++ & - & - & - & - & $\ldots$ & - & - & - & - & - & - & + \\
\hline COMT & +++ & +++ & +++ & +++ & +++ & ++ & ++ & +++ & ++ & ++ & +++ & ++ & +++ & +++ & ++ \\
\hline$G G T 1$ & + & + & +++ & - & + & $\ldots$ & ++ & ++ & +++ & + & ++ & ++ & ++ & ++ & ++ \\
\hline GLYAT & - & $\ldots$ & - & - & + & - & - & - & - & - & - & - & - & - & - \\
\hline
\end{tabular}


Table 8 (continued)

\begin{tabular}{|c|c|c|c|c|c|c|c|c|c|c|c|c|c|c|c|}
\hline \multirow[t]{2}{*}{ Gene } & \multicolumn{11}{|c|}{ Lung cell models } & \multicolumn{4}{|c|}{ Lung tissues } \\
\hline & $16 \mathrm{HBE}$ & 1HAE0 & A549 & BEAS-2B & Calu-1 & L-132 & $\mathrm{H} 292$ & H358 & H460 & H727 & HBEC & $\mathrm{BM}$ & PP & $\mathrm{SCC}$ & $\mathrm{AC}$ \\
\hline GNMT & - & - & - & + & - & - & - & - & - & - & - & + & + & - & - \\
\hline GSTA1 & - & - & + & - & - & - & - & - & - & - & + & +++ & ++ & ++ & + \\
\hline GSTA2 & - & - & + & - & - & - & - & - & - & - & - & +++ & + & + & $\ldots$ \\
\hline GSTA3 & - & - & - & - & - & - & - & - & - & - & - & ++ & + & - & - \\
\hline GSTA4 & ++ & + & ++ & ++ & ++ & + & + & ++ & ++ & ++ & ++ & ++ & +++ & ++ & ++ \\
\hline GSTA5* & - & - & - & - & - & - & - & - & - & - & - & - & - & - & - \\
\hline GSTK1 & +++ & +++ & +++ & +++ & +++ & ++ & +++ & ++ & ++ & ++ & +++ & +++ & +++ & +++ & +++ \\
\hline GSTM1 & - & - & - & - & ++ & - & - & - & + & - & \pm & - & - & - & - \\
\hline GSTM2 & - & - & ++ & ++ & ++ & - & + & + & ++ & + & + & ++ & ++ & ++ & ++ \\
\hline GSTM3 & - & ++ & ++ & ++ & ++ & ++ & + & + & +++ & + & ++ & ++ & ++ & ++ & ++ \\
\hline GSTM4 & ++ & ++ & ++ & ++ & ++ & ++ & ++ & + & ++ & + & + & ++ & ++ & ++ & ++ \\
\hline GSTM5 & - & - & - & - & + & - & - & - & + & - & - & ++ & ++ & + & + \\
\hline GSTOI & +++ & +++ & +++ & +++ & +++ & ++ & +++ & ++ & +++ & +++ & +++ & ++ & +++ & +++ & ++ \\
\hline GSTO2 & ++ & ++ & + & ++ & + & - & ++ & ++ & ++ & ++ & ++ & ++ & + & ++ & + \\
\hline GSTP1 & +++ & +++ & +++ & +++ & +++ & +++ & +++ & +++ & +++ & +++ & +++ & +++ & +++ & +++ & +++ \\
\hline GSTT1 & +++ & ++ & - & - & - & + & ++ & ++ & ++ & ++ & + & + & ++ & ++ & + \\
\hline GSTT2/2B & ++ & +++ & + & ++ & ++ & + & ++ & + & + & $\ldots$ & + & ++ & ++ & ++ & ++ \\
\hline GSTZ1 & ++ & +++ & +++ & ++ & ++ & + & ++ & ++ & ++ & ++ & ++ & ++ & ++ & ++ & ++ \\
\hline$H N M T$ & - & - & ++ & + & +++ & + & ++ & ++ & ++ & ++ & + & +++ & +++ & ++ & +++ \\
\hline INMT & - & - & - & + & - & - & - & - & - & $\ldots$ & - & ++ & +++ & + & ++ \\
\hline$M G S T 1$ & +++ & +++ & +++ & +++ & +++ & +++ & +++ & +++ & +++ & ++ & +++ & +++ & +++ & +++ & +++ \\
\hline$M G S T 2$ & ++ & ++ & +++ & +++ & ++ & ++ & +++ & ++ & +++ & ++ & ++ & +++ & ++ & ++ & ++ \\
\hline MGST3 & +++ & ++ & +++ & +++ & +++ & ++ & +++ & ++ & +++ & +++ & +++ & +++ & +++ & +++ & +++ \\
\hline$M P S T$ & +++ & +++ & +++ & +++ & +++ & ++ & +++ & ++ & +++ & ++ & +++ & ++ & +++ & +++ & ++ \\
\hline NAT1 & + & + & + & + & + & - & + & + & - & $\ldots$ & - & + & - & + & - \\
\hline NAT2 & - & - & - & - & $\ldots$ & - & - & - & - & - & - & - & - & - & - \\
\hline NAT5 & +++ & +++ & +++ & +++ & +++ & ++ & +++ & +++ & +++ & ++ & +++ & +++ & ++ & +++ & +++ \\
\hline$N N M T$ & ++ & ++ & +++ & ++ & ++ & ++ & +++ & - & + & +++ & ++ & ++ & +++ & ++ & +++ \\
\hline$P N M T$ & - & - & - & - & - & - & - & $\ldots$ & - & + & - & + & + & - & - \\
\hline SULT1A1 & - & + & - & $\ldots$ & - & $\ldots$ & - & - & - & - & - & + & - & - & - \\
\hline SULT1A2 & - & + & - & + & - & - & + & $\ldots$ & - & - & + & + & ++ & + & + \\
\hline SULT1A3/4 & ++ & ++ & ++ & ++ & ++ & + & ++ & + & + & + & ++ & ++ & ++ & ++ & ++ \\
\hline SULT1B1 & - & - & - & - & ++ & - & - & - & - & - & + & + & + & + & + \\
\hline SULT1C2 & - & - & + & - & + & - & - & - & - & + & - & - & + & - & ++ \\
\hline SULT1C $3 *$ & - & - & - & - & - & - & - & - & - & - & - & - & - & - & - \\
\hline SULT1C4 & - & - & - & - & - & - & - & - & - & - & - & ++ & ++ & + & + \\
\hline SULT1E1 & - & - & - & + & - & - & + & + & - & - & + & ++ & + & + & - \\
\hline$S U L T 2 A 1^{*}$ & - & - & - & - & - & - & - & - & - & - & - & - & - & - & - \\
\hline$S U L T 2 B 1$ & +++ & + & +++ & - & - & - & ++ & ++ & - & $\ldots$ & +++ & ++ & ++ & ++ & ++ \\
\hline SULT4AI & - & ++ & - & - & - & + & - & - & - & - & + & + & - & + & - \\
\hline SULT6B1 & - & - & - & - & - & - & - & - & + & - & - & - & - & - & - \\
\hline$T P M T$ & +++ & +++ & +++ & +++ & +++ & ++ & ++ & ++ & ++ & ++ & +++ & ++ & ++ & ++ & +++ \\
\hline$T S T$ & +++ & ++ & +++ & ++ & ++ & ++ & ++ & ++ & ++ & ++ & ++ & ++ & ++ & ++ & ++ \\
\hline UGT1A1 & - & + & ++ & - & - & $\ldots$ & - & - & $\ldots$ & + & + & ++ & + & ++ & + \\
\hline UGT1A10 & - & - & - & - & - & - & - & - & - & - & \pm & ++ & - & ++ & + \\
\hline UGT1A4 & - & - & - & - & - & - & - & - & - & - & - & + & - & ++ & $\ldots$ \\
\hline UGT1A5 & - & - & - & - & - & - & - & - & - & - & - & + & - & + & + \\
\hline UGT1A6 & - & ++ & ++ & - & - & +++ & - & - & - & - & ++ & ++ & - & ++ & + \\
\hline
\end{tabular}


Table 8 (continued)

\begin{tabular}{|c|c|c|c|c|c|c|c|c|c|c|c|c|c|c|c|}
\hline \multirow[t]{2}{*}{ Gene } & \multicolumn{11}{|c|}{ Lung cell models } & \multicolumn{4}{|c|}{ Lung tissues } \\
\hline & $16 \mathrm{HBE}$ & 1HAE0 & A549 & BEAS-2B & Calu-1 & L-132 & $\mathrm{H} 292$ & $\mathrm{H} 358$ & $\mathrm{H} 460$ & $\mathrm{H} 727$ & HBEC & $\mathrm{BM}$ & $\mathrm{PP}$ & $\mathrm{SCC}$ & $\mathrm{AC}$ \\
\hline UGT1A7 & - & - & +++ & - & - & - & - & - & - & - & - & ++ & - & ++ & + \\
\hline UGT1A8 & - & - & ++ & - & - & - & - & - & - & - & \pm & + & - & + & - \\
\hline UGT1A9 & - & - & ++ & - & - & - & - & - & $\ldots$ & - & - & + & - & + & - \\
\hline$U G T 2 A 1$ & - & - & - & - & - & - & - & - & - & - & - & ++ & - & - & - \\
\hline$U G T 2 A 3$ & - & - & ++ & - & - & - & - & - & - & - & - & - & - & - & - \\
\hline$U G T 2 B 10$ & - & - & + & - & $\ldots$ & - & - & - & - & $\ldots$ & - & - & - & + & - \\
\hline$U G T 2 B 11$ & - & - & ++ & - & - & - & - & - & $\ldots$ & - & - & + & + & + & + \\
\hline$U G T 2 B 15$ & - & - & + & - & - & - & - & $\ldots$ & - & $\ldots$ & - & + & - & + & + \\
\hline$U G T 2 B 17$ & - & - & - & - & - & - & - & + & - & - & - & + & - & + & + \\
\hline$U G T 2 B 28$ & - & - & + & - & - & - & - & - & - & - & - & + & - & + & - \\
\hline$U G T 2 B 4$ & - & - & + & - & - & - & - & - & - & - & - & - & + & - & - \\
\hline$U G T 2 B 7$ & - & - & ++ & - & - & - & - & - & - & + & - & - & - & - & - \\
\hline$U G T 3 A 1 *$ & - & - & - & - & - & - & - & - & - & - & - & - & - & - & - \\
\hline UGT3A2 & - & + & - & ++ & - & - & + & - & - & - & - & + & - & - & + \\
\hline UGT8 & - & - & - & - & +++ & ++ & - & ++ & ++ & + & - & ++ & + & ++ & ++ \\
\hline
\end{tabular}

$A C$ adenocarcinoma, $B M$ bronchial mucosa, $H B E C$ human bronchial epithelial cell, $P P$ pulmonary parenchyma, $S C C$ squamous cell carcinoma - , not detectable $(\beta \mathrm{Ct}>26)$; ..., very low $(26>\beta \mathrm{Ct}>24) ;+$, low $(24>\beta \mathrm{Ct}>20) ;++$, moderate $(20>\beta \mathrm{Ct}>16)$;,+++ high $(\beta \mathrm{Ct}<16) ;+/-$, variable

*Not detectable in all models

correlated positively with the expression of CYP1A1 mRNA in the lung of both, males and females (Mollerup et al. 1999). Interestingly, a relatively small subset of smokers (10-20\%) did not have measurable amounts of CYP1A1 mRNA in the lung (nor CYP1A1 protein or activity) (Hukkanen et al. 2002). This lack of inducibility by tobacco smoke constituents is not explained by polymorphisms of AhR (aryl hydrocarbon receptor), ARNT (AhR translocator) or CYP1A1 (Anttila et al. 2000). AhR and ARNT, which are involved in the regulation of CYP1A1 expression, have both been identified in human lung (Hukkanen et al. 2002). Cigarette smoke contains many AhR agonists such as benzo[a]pyrene and further polycyclic aromatic hydrocarbons. The localization of CYP1A1 mRNA generally corresponded well with that of CYP1A1 protein (Saarikoski et al. 1998). CpG sites up to $1.4 \mathrm{~kb}$ upstream of the CYP1A1 promoter were partially or completely methylated in practically all (98\%) nonsmokers, but only in 33\% of heavy smokers (Anttila et al. 2003).

CYP1B1 mRNA also is expressed in human lung (Sutter et al. 1994; Willey et al. 1996; Shimada et al. 1996a). Levels of CYP1B 1 transcripts in human lung were elevated in smokers as shown by Port et al. (2004) in smokers bronchial mucosa. Takahashi et al. (2018) found that CYP1B1 transcripts were increased in bronchial brushings from currently smoking asthmatics compared with ex-smoking and non-smoking asthmatics and non-smoking healthy individuals. Conversely, Walters et al. (2017) found that in bronchial brushings obtained from small airway epithelium of individuals smoking water pipe (a common form of tobacco use in the Middle East) CYP1B1 transcripts were reduced compared with non-smokers, but the $C Y P 1 B 1$ gene was hypomethylated.

Chang et al. (2006) showed that Clara cells were highly sensitive to TCDD and benzo[a]pyrene as inducers of CYP1A1, CYP1B1 and AhR mRNA (and protein).

CYP2A6 mRNA is expressed in human bronchial epithelium (Crawford et al. 1998; Macé et al. 1998). Tobacco smoking represses the expression of CYP2A6 transcript in human bronchial epithelial cells (Crawford et al. 1998). Su et al. (2000) reported high expression of CYP2A13 mRNA in human lung (of 17 investigated tissues second highest after nasal mucosa).

CYP2B6 gene is expressed in human lung as a splicing variant, which previously has been called CYP2B7. Czerwinski et al. (1994) detected "CYP2B7" and CYP4B1 transcripts in normal human lung and in human lung cancer tissues, in the former at a higher levels. CYP2B6 mRNA also was detected in human lung by Gervot et al. (1999).

Macé et al. (1998) found 2C8 and 2C18 transcripts in the human bronchus as well as in the peripheral lung tissue.

Guidice et al. (1997) found CYP2D6 transcripts in human lung, the levels in comparison with the liver being 3 times 
lower in the bronchial mucosa and 6 times lower in the lung parenchyma.

Nhamburo et al. (1990) were first to identify a very low mRNA level of the lung-selective CYP2F1 in human lung tissue. Raunio et al. (1998) confirmed the presence of the mRNA for CYP2F1 in the human lung. Because of the CYP2F-mediated species differences in susceptibility to quite a number of pneumotoxins it is worthy of note that the human CYP2F1 cDNA has a $82 \%$ identity with the CYP2F2 of the mouse which is highly susceptible to many of these pneumotoxins.

Zeldin et al. (1996) discovered the presence of CYP2J2 mRNA in human lung.

Rylander et al. (2001) observed mRNA for the novel CYP2S1 in human lung.

Kivisto et al. (1995) reported CYP3A5 and 3A7, but not 3A4 transcripts expressed in normal and in tumorous human lung tissue. Anttila et al. (1997) observed CYP3A5 mRNA in the lung of all eight human individuals investigated, CYP3A4 in only one and CYP3A7 in none of them. Westlind et al. (2001) observed in the human lung CYP3A4 transcripts, albeit at a very low level. CYP3A5 mRNA was present in human alveolar macrophages and was repressed by tobacco smoking (Hukkanen et al. 2002). mRNA for CYP3A43 were observed in one (Westlind et al. 2001), but not in another investigation (Domanski et al. 2001).

Willey et al. (1996) reported the presence of quite a number of CYP transcripts in human bronchial epithelial cells: CYP1A1, 1B1, 2B7, 2E1, 2F1 and 4B1; CYP1A1 and 1B1 more highly expressed in smokers compared with nonsmokers; no difference between smokers and non-smokers were noted for CYP2B7 transcripts. Hukkanen et al. (1997) observed expression of mRNA for several CYPs in human lung as well as human bronchoalveolar macrophages. CYP1A1, 2B6/7, 2E1, 2F1, 3A and 4B1 were observed in whole lung tissue, CYP2B6/7, CYP2C, CYP2E1, CYP2F1, CYP3A5 and CYP4B1 in bronchoalveolar macrophages. Crawford et al. (1998) reported the presence of CYP2A6 and 2E1 mRNA in human bronchial epithelial cells.

Macé et al. (1998) investigated CYP transcript expression in human lung samples of 14 individuals. In the bronchial mucosa specimens CYP1A1, 2C8 and 2C18 transcripts were expressed in all samples, CYP3A5 transcripts in 90\%, CYP2A6 and 2B6 transcripts in 85\% and CYP2E1 transcripts in 50\% of them, CYP1A2, 2D6 and 3A4 in none of them. CYP transcript expression was similar in peripheral lung tissue except that there CYP1A2 and 3A4 transcripts have been expressed.

Vieira et al. (1998) observed CYP2E1 mRNA at comparable, but very low levels in foetal, newborn and adult human lung. CYP2E1 expression in the human lung was under the control of CpGs methylation of the CYP2E1 gene.
The very low expression of CYP2E1 mRNA in human lung (as opposed to liver) corresponded to a very heavy methylation of HpaII/MspI sites within the promoter, the first exon and first intron of the CYP2E1 gene (Vieira et al. 1998). On the other hand, low expression of the CYP2E1 gene in lung tumor was associated with hypomethylation of the CYP2E1 gene (Botto et al. 1994).

Choudhary et al. (2005) found that the predominant expression of the mRNA for different CYPs in the human late-term fetus was organ-selective: in the foetal lung highest for CYP4B1 and 2W1 (as compared to liver: highest for CYP2E1, thymus: highest for CYP1B1 and 2U1, brain: highest for CYP1A1 and 2R1).

Ling et al. (2007) using a 216-base pair CYP2A13 promoter fragment demonstrated that the expression of transcripts coding for the respiratory-tract selective CYP2A13 is regulated by CCAAT/enhancer binding protein (C/EBP) transcription factors. In NCI-H441 human lung cancer cells CYP2A13 expression was increased by overexpression of $\mathrm{C} /$ EBP delta and decreased by small interfering RNA-mediated suppression of C/EBPdelta.

Bieche et al. (2007) investigated the expression of CYP transcripts in 22 different human tissues. CYP1A1 mRNA was higher in lung than in any other tissue, and CYP1B1 and 2B6 transcripts were relatively high in lung. CYP2F1 mRNA was found exclusively in the lung. The lung-selective expression of CYP2F1 is due to SP1-dependent proximal promoter elements and the presence of $\mathrm{Sp} 1$ and $\mathrm{Sp} 3$ transcription factors in lung cells (Wan et al. 2005) and to a lung-specific factor ("LSF") which binds to a lung-specific binding motif in the 5 '-upsteam region of the CYP2F1 promoter (Carr et al. 2003a).

Some confusion arose on human lung CYP4B. Nhamburo et al. (1989) had reported the discovery of a new CYP expressed in human lung at the mRNA level, which they called CYP IVB1 (CYP4B1). The cDNA inserted into a vaccinia virus expression vector followed by expression of the enzyme in human cell lines - in contrast to the related rabbit CYP ("P450 isozyme 5", now called CYP4B1)—did not activate 2-aminofluorene nor did human lung microsomes catalyze this reaction despite the presence of CYP IVB1 mRNA in 3 out of 4 human lungs (CYP IVB 1 transcripts were not detected in the human liver). The recombinant enzyme also did not metabolize 4-ipomeanol (Czerwinski et al. 1991), a prototypic substrate for rabbit CYP4B1. On the other hand, Waxman et al. (1991) reported the observation of typical CYP3A activities such as $6 \beta$-hydroxylation of testosterone, androstenedione and progesterone using this "CYP4B1" protein. The results were finally explained by a CYP3A5 contamination in the protein preparations (Zheng et al. 1998). Recently Choudhary et al. (2005) reported that among human organs CYP4B1 transcripts were found predominantly in the lung ( $70 \%$ of organ distribution, the 
remainder in heart, skeletal muscle and kidney; none in the liver).

Zuo et al. (2018) demonstrated in samples_-gained by bronchoscopy and by scraping from the human small airways epithelial Clara (Club) cells - transcripts of the following CYPs: 2F1 (very abundant), 4B1 (abundant), 2J2, 51A1, as well as CYB5R3 (cytochrome b5 reductase 3).

Up versus down regulation of the expression of CYP transcripts by cigarette smoke (as seen by comparison between smokers and nonsmokers) was differential for individual CYPs (Thum et al. 2006): in bronchus biopsies of smokers CYP1A1, 1B1 and 2C9 transcripts were induced, CYP2J2 transcripts were repressed. In bronchoalveolar lavage cells of smokers CYP1A1, CYP1B1, CYP2S1, LXR (liver X receptor) and GR (glucocorticoid receptor) transcripts were induced, CYP2B6/7 and CYP3A5 transcripts were repressed [the repression of CYP3A5 had already been observed by Hukkanen et al. (2003) in alveolar macrophages of smokers]. The induction of GR transcripts (in bronchoalveolar lavage cells) and the induction of CYP2C9 transcripts (in bronchus biopsies) in the study by Thum et al. (2006) may be related since CYP2C9 had been shown to be a glucocorticoid target gene (Gerbal-Chaloin et al. 2002).

Leclerc et al. (2011) found in healthy human bronchial mucosa and in pulmonary parenchyma, respectively, the following CYP transcripts at the following expression levels (Median DCt):

CYP1B1 16.3; 15.9

CYP2B6 16.6; 15.9

CYP2F1 15.5; 21.9

CYP2S1 $16.9 ; 18.7$

CYP4B1 13.3; 13.1

CYP4X1 $15.4 ; 17.4$

CYP8A1 17.2; 15.4

CYP27A1 17.0; 15.6.

Quite a number of CYPs mRNAs were not detected-neither in nonsmokers $(n=10)$ nor in smokers $(n=8)$, neither in bronchial biopsies nor in alveolar lavage cells-in the study of Thum et al. (2006): CYP1A2, 2C8, 2C18, 2C19, 2D6, 3A7, 4A11 (in the same study mRNAs of several other CYPs were detected, see just above). However, Somers et al. (2007) did detect mRNA of some of these in the human lung parenchyma: CYP1A2, 2C19, and 2D6.

\section{CYP and CYP reductase proteins expression}

CYP reductase protein was observed in human lung bronchial and bronchiolar epithelia, alveolar wall and alveolar macrophages (Hall et al. 1989).

Reports on individual CYPs proteins in the human lung are partly conflicting. One reason for the conflicting reports on the expression of various CYPs on the protein level may be the very low levels of total CYP in the human lung. Thus, McManus et al. (1980) reported that human lung microsomes did not contain detectable CYP. Subsequently, the levels of total CYPs in the human lung was variously reported to be between 2 and $10 \mathrm{pmol} / \mathrm{mg}$ microsomal protein. This concentration is much lower than in the lung of mouse, rat, rabbit, hamster, guinea pig or dog (Bond 1993), about 20-fold lower than in the rat lung and about 100-fold lower than in the rabbit lung (Hukkanen et al. 2002). Almost all xenobiotica-metabolizing CYPs are much lower in the human lung compared with the human liver, with notable exceptions including CYP2A13, 2F1, 2S1 and 3A5.

Nakajima et al. (1994) noticed in human lung tissueusing immunochemistry-faint bands upon treatment with antibodies against quite a number of CYP proteins: CYP1A2, 2B1, 2C9, 2E1 and 3A1, but not CYP2B6 or 2C8. Shimada et al. (1996a) also reported in the human lung the presence of quite a number of CYPs immunoreactive with the following anti-human CYP antibodies: CYP1A1, 2A6, 2C9, 2E1 and 3A4. Bernauer et al. (2006) also obtained evidence for the presence in the human lung of quite a number of CYP proteins cross-reactive with anti-CYP antibodies: firm evidence for the presence of CYP1A1, 1A2, 2E1 and 3A5; suggestive evidence for the presence of CYP1B1, 2A, 2B6, 2C, 2D6, and 3A4.

Evidence for the presence of individual CYPs in the human lung was found as follows.

CYP1A1 protein expression was reported to occur in human lung, but only in the epithelium of peripheral (small) airways, not in the epithelium of bronchi larger than $1 \mathrm{~mm}$ in diameter, not in alveolar macrophages and not in the lung of non-smokers (Wheeler et al. 1990). Using Western blotting Anttila et al. (1992, 2001) also observed CYP1A1 protein in human lung of smokers, but not in non-smokers. On the other hand, a relatively small subset of smokers (10-20\%) does not have measurable amounts of CYP1A1 protein in the lung (nor CYP1A1 mRNA or activity) (Hukkanen et al. 2002). However, in all investigated cases of peripheral lung cancer CYP1A1 protein was expressed and was, therefore, hypothesized to possibly be a prerequisite for its development (Zhang et al. 2006). Shimada et al. (1992) confirmed the presence of CYP1A1 protein in human lung, but levels were much lower than in the liver. The localization of CYP1A1 protein corresponded well with the expression of CYP1A1 mRNA (Saarikoski et al. 1998).

CYP1B1 protein also was detected in human lung (Tang et al. 1999; Spivack et al. 2001). Piipari et al. (2000) observed CYP1B1 protein in 13/31 bronchoalveolar macrophage samples from tobacco smokers and only in $3 / 16$ samples from non-smokers, while Chang et al. (2006) showed that Clara (Club) cells were highly sensitive to the CYP1A1, CYP1B1 and AhR protein (and mRNA) inducing effect of TCDD and benzo[a]pyrene. Kim et al. (2004) confirmed the presence of CYP1A1 and 1B1 protein in human 
lung (with high interindividual difference) and its increase by cigarette smoking ( $15.5 \mathrm{pmol}$ CYP1A1 protein per $\mathrm{mg}$ microsomal protein in smokers, $6 \mathrm{pmol} / \mathrm{mg}$ microsomal protein in non-smokers; $1.8 \mathrm{pmol} \mathrm{CYP1B1}$ protein per $\mathrm{mg}$ microsomal protein in smokers and $1.0 \mathrm{pmol} / \mathrm{mg}$ microsomal protein in nonsmokers). They localized CYP1A1 and 1B1 protein to alveolar type I and type II pneumocytes, ciliated columnar epithelial cells lining bronchoalveolar airways as well as alveolar macrophages. Iba et al. (2010) reported the increase of CYP1A1 and 1A2, but not 1B1 protein levels in human lung samples exposed to BP or to diesel exhaust particle extract.

Zhu et al. (2006) developed an antibody which highly specifically recognized CYP2A13 and not the closely related (93.5\% amino acid identity) CYP2A6 (important because of the high activity of CYP1A13 toward aflatoxin B1 and the lung carcinogen NNK). Using this antibody they detected a high expression of CYP2A13 in human bronchus and trachea, and very little expression in alveolar cells leading to their suggestion that this localization may substantially contribute to the fact that most smoking-related human lung cancers are bronchogenic. CYP2A6 and CYP2A13 proteins were detected by Chiang et al. (2012) in epithelial cells of the human trachea and bronchus, but in bronchiolar epithelia they observed only CYP2A6 protein (with the additional limitation that the antibody used for detecting CYP2A6 was cross-reactive with the non-functional CYP2A7 protein).

CYP2B6 protein was detected in human lung by Gervot et al. (1999) after developing a very specific antibody against CYP2B6, but its levels were much lower than in other tissues such as liver, kidney or intestine.

Yokose et al. (1999) observed CYP2C protein in human lung bronchial gland serous cells, but in no other human lung tissue.

CYP2D protein expression in human lung is very low or nil (Hukkanen et al. 2002) [while CYP2D6 mRNA has been observed in the human lung in some, but not in other studies (see above)].

Using immunochemical methods De Waziers et al. (1990) observed CYP2E1 protein in human lung. Runge et al. (2001) found in primary cultures of human bronchial epithelial cells low levels of CYP2E1 protein.

Somewhat later Wheeler et al. (1992) reported that they observed CYP2E1 and CYP3A, but not CYP2C8 or CYP2C9 proteins in human lung.

Zeldin et al. (1996) observed CYP2J2 protein in human lung tissues primarily in ciliated epithelial airway cells, but also in nonciliated epithelial airway cells, in the lung vascular endothelium and alveolar macrophages, much higher in the alveolar epithelium than in endothelial and vascular smooth muscle cells.

Rylander et al. (2001) observed the novel CYP2S1 protein in human lung, which was confirmed and extended by
Saarikoski et al. (2005) identifying this CYP protein at high levels in bronchi, bronchioli and nasal cavity.

Kivisto et al. (1995) determined immunohistochemically CYP3A and 2E1 protein in both, human lung cancer and normal human lung tissue and they observed the presence of CYP3A5 and 3A7 protein in normal and in tumorous human lung tissue (Kivisto et al. 1995). Shimada et al. (1996a) confirmed the presence of CYP1A1, 2E1 and 3A4 protein in human lung; in contrast to the lack of finding CYP2A6 protein by Macé et al. (1998) in human peripheral lung tissue, they noticed CYP2A6 protein in human whole lung and extended the list by the observation of CYP2C9 protein in human lung.

Kelly et al. (1997) observed CYP3A protein, but no CYP1A2 protein in human lung. In the subsequent year Anttila et al. (1997) reported the finding of CYP3A4 protein in human lung, but in contrast to the liver mostly 3A5. This CYP form (3A5) was localized widely in the lung: in bronchial ciliated, mucous and glandular cells, bronchiolar columnar and terminal cuboidal epithelium, type I and type II alveolar cells, vascular and capillary endothelium and alveolar macrophages (CYP3A4 in bronchial glands, bronchiolar columnar and terminal epithelium, type II alveolar cells and alveolar macrophages). CYP3A5 protein was found in all individuals tested, CYP3A4 only in $20 \%$ of them (Raunio et al. 1999). Macé et al. (1998) observed in human peripheral lung tissue CYP3A4/5 as well as CYP1A1, 1A2, 2B6 and 2E1, but not 2D6 proteins. Van Vleet et al. (2001) observed 3A4 and CYP1A in NHBE (normal human bronchial epithelial cells). Raunio et al. (2004) observed in lung adenocarcinoma derived A549 cells induction of CYP3A5 protein by glucocorticoids and, interestingly, in alveolar macrophages repression by cigarette smoke. In freshly isolated bronchoalveolar macrophages and/or A549 cells proteins immunologically related to CYP3A were observed in human fetal lung (Hakkola et al. 1998).

Toussaint et al. (1993) observed CYP1A1/1A2 protein in human pulmonary normal and tumor tissue, but CYP2B1/2B2, 2C8, 2E1 or 3A4 in neither of them.

Taken together, CYP protein expression in the human lung is in general much lower than in the liver, but a considerable number of CYPs proteins are present in the human lung. Preferential expression of CYP proteins in the human lung includes CYP1A1, 1B1, 2A13, 2E1, 2F1, 2S1, 3A5 and 4B1. An important difference in the localization of CYPs in the human lung compared with the lung of laboratory rodents is their weaker expression in Clara cells and the considerably lower number of Clara cells in the human lung and, in addition, their further reduction by tobacco smoking (Hukkanen et al. 2002). This is an especially important difference, since Clara cells (at least in rodents) are one primary origin and location of lung toxicity evoked by several xenobiotic compounds. 


\section{CYP catalytic activities (Table 3 )}

A major difficulty in studies on CYP catalytic activities in the human lung is their very low levels. Total human pulmonary CYP protein on a per milligram of protein basis is about 20 times less than in the rat lung and about 100-fold less than in the rabbit lung (Wheeler and Guenthner 1991). Nevertheless, despite their low levels, several pulmonary CYPs are involved in major toxication reactions and are important for local lung toxicities. Several pneumonal CYPs are involved in the activation of quite a number of procarcinogens by formation of reactive metabolites derived from, e.g. polycyclic aromatic hydrocarbons and $N$-nitrosamines (Hecht 1999). The essential role of CYPs in the activation of the lung-specific carcinogen NNK [4-methylnitrosamino)-1(3-pyridyl)-1-butanone] was proven by the use of lung CYP reductase null mice abolishing all CYP mediated activities (Weng et al. 2007). Examples involving individual CYPs are presented below.

Aryl hydrocarbon hydroxylase (AHH) Wheeler et al. (1990) detected in human lung microsomes AHH activities which were on a per $\mathrm{mg}$ protein basis 2-fold slower than in rat lung. Shimada et al. (1992) also observed AHH activity in human lung microsomes, 14-fold lower than in rat lung. Bartsch et al. (1992) reported AHH activity in the lungs of smokers in good agreement with the levels of CYP1A1 protein.

Prototypical CYP catalytic activities other than AHH Very early on Lorenz et al. (1979) already observed considerable activities of the broad-spectrum CYP activity 7-ethoxycoumarin $O$-deethylase (ECOD) in human lung microsomes, statistically not different between cancer patients and non-cancer (Table 3). Rates in human lung microsomes were 57-fold lower as compared with rat lung microsomes (Oesch et al. 1980). Pacifici et al. (1988b) confirmed activity of human lung microsomes toward the broad-specific prototype substrate 7-ethoxycoumarin.

Wheeler et al. (1990) detected in human lung the CYP1 prototypic 7-ethoxyresorufin O-deethylase (EROD) as well as ECOD activities which were on a per mg protein basis 4- and 34-fold slower than in rat lung. Shimada et al. (1992) observed very low levels of EROD and CYP 2B-prototypic PROD (7-pentoxyresorufin O-dealkylase) in human lung microsomes, 22- and 3-fold lower, respectively, than in rat lung microsomes (smoker status not reported). Anttila et al. (1992) observed EROD activity in the lung of smokers, but not in the lung of non-smokers. Bernauer et al. (2006) reported quite remarkable CYP1A2-prototypic MROD (7-methoxyresorufin O-dealkylase) activities in human lung microsomes, which were in the $4-11 \mathrm{nmol} / \mathrm{min} / \mathrm{mg}$ microsomal protein range. However, Donato et al. (1999) observed
EROD and MROD activities only in the lung samples from smokers.

Iba et al. (2010) observed in unbroken human lung ex vivo samples EROD, MROD and the CYP1 prototypic CECOD (3-cyano-7-ethoxycoumarin $O$-deethylase) activities, which were not substantially inhibited by the CYP1B1preferential inhibitor tetramethoxystilbene. The authors concluded that it is unlikely that CYP1B1 contributes to any of the three activities in the human lung. CECOD activity was markedly inhibited by the CYP1A2-preferential inhibitor fluvoxamine indicating substantial contribution of CYP1A2 to CECOD activity in the human lung.

CYP2A6-prototypic coumarin 7-hydroxylation was observed in lung samples of all human individuals investigated (Donato et al. 1999).

Shimada et al. (1992) reported the presence of the CYP2B6-prototypic PROD (7-pentoxyresorufin depentylase) activity in human lung microsomes, about 5-fold lower than in the human liver and about 3-fold lower than in the rat lung. Donato et al. (1999) observed PROD activity in all human lung samples investigated.

CYP2D6-prototypical bufuralol hydroxylation was not detected in human lung (Hukkanen et al. 2002) in agreement with very low or nonexistent CYP2D protein expression in human lung (see above).

CYP2E1-prototypical hydroxylation of 4-nitrophenol has been observed in human lung microsomes (Forkert et al. 2000). CYP2E1-prototypical NDMA ( $N$-nitrosodimethylamine) demethylation in human lung microsomes ranged from 36.9 to $82.4 \mathrm{pmol} / \mathrm{mg}$ protein $/ \mathrm{min}$ (Forkert et al. 2001). Bernauer et al. (2006) observed CYP2E-prototypic chlorzoxazone metabolism in human lung microsomes, about 100 times slower than in human liver microsomes. The activity varied about 20-fold between individuals (Bernauer et al. 2005). However, chlorzoxazone hydroxylation has not been observed in human lung microsomes by Uehara et al. (2018) at their limit of detection at $12 \mathrm{pmol} / \mathrm{min} / \mathrm{mg}$ microsomal protein.

Donato et al. (1999) observed the CYP3A-prototypic testosterone $6 \beta$-hydroxylation in the lung samples from all human individuals investigated.

Shimada et al. (1996a) monitored activity in human lung microsomes with several substrates and compared them with human liver microsomes, in all cases finding much lower activities in the lung: EROD specific activity (i.e. activity on a per mg protein basis) was 69 times lower in the lung; lung coumarin hydroxylase $<0.05 \mathrm{pmol} / \mathrm{mg}$ protein $/ \mathrm{min}$, liver $44 \mathrm{pmol} / \mathrm{mg}$ protein $/ \mathrm{min}$; lung ECOD $<0.2 \mathrm{pmol} / \mathrm{mg}$ protein $/ \mathrm{min}$, liver $38 \mathrm{pmol} / \mathrm{mg}$ protein/min; lung testosterone $6 \beta$-hydroxylase $0.26 \mathrm{pmol} / \mathrm{mg}$ protein/min, liver $2756 \mathrm{pmol} /$ $\mathrm{mg}$ protein/min. 
Pulmonary CYPs in the metabolism of styrene Because of the species-specific carcinogenicity of styrene (carcinogenic in the mouse lung, but not in the rat) (Ponomarkov and Tomatis 1978; U. S. NCI 1979; Cruzan et al. 1998, 2001, 2002) the metabolism of styrene in the human lung compared with the rat versus the mouse lung is of special interest. The complex metabolism of styrene in man contrasted with experimental animal species has been reviewed by Vodicka et al. (2006). Important data concerning human pulmonary CYP-mediated metabolism are detailed below:

Guengerich et al. (1991) showed that purified CYP2E1 metabolized styrene to the reactive genotoxin styrene 7,8-oxide.

Nakajima et al. (1994) observed that the sequence of styrene metabolism by 12 recombinant CYPs which occur in the human lung and/or liver was as follows: CYP2B6 and $2 \mathrm{E} 1$ occurring in the human liver and/or lung and CYP2F1 occurring in the human lung were fastest, followed by CYP1A2 and 2C8, while CYP3A3, 3A4, 3A5 and 4B1 were much slower and CYP2A6, 2C9 and 2D6 were almost inactive. Moreover they reported that the rate of glycol formation from styrene was higher in lung microsomes from smokers compared with current nonsmokers.

Fukami et al. (2008) compared the activity of purified CYP2A13 and CYP2E1 for styrene metabolism and found the former to be more active.

Nakajima et al. (1994) reported for human lung microsomes a rate of pulmonary biotransformation of styrene to styrene glycol (rightfully presumed to be via styrene 7,8 -oxide) of $0.13 \mathrm{nmol} / \mathrm{mg}$ protein per $20 \mathrm{~min}$ [assuming linearity with respect to time this would correspond to $0.0065 \mathrm{nmol} / \mathrm{mg}$ protein/min, much less than for mice: $2.06 \mathrm{nmol} / \mathrm{mg}$ protein/min for CD-1 mouse lung microsomes (Carlson 1997) and $2.7 \mathrm{nmol} / \mathrm{min} / \mathrm{mg}$ protein for B6C3F1 mouse lung microsomes (Mendrala et al. 1993)]. Using the kinetic constants obtained by these authors (Mendrala et al. 1993), Sarangapani et al. (2002) carried out PBPK modeling leading to predicting about 100-fold lower styrene 7,8-oxide concentration in the human terminal bronchioles compared with the mouse, the species in which lung tumors had been observed upon exposure to styrene (Cruzan et al. 2001) (in the rat where upon exposure to styrene no lung tumors had been observed the predicted styrene 7,8-oxide concentrations in the terminal bronchioles were about 10-fold lower compared with the mouse).

Carlson et al. (2000) reported that lung samples of only 1 out of 6 subjects were able to metabolize styrene to styrene 7,8-oxide (while the liver samples of all 6 subjects were able to perform this metabolic step), and this metabolism in the human lung was very slow $(0.088 \mathrm{nmol} / \mathrm{mg}$ protein $/ \mathrm{min}$; much slower than in the mouse lung).
One crucial difference between the human, rat and mouse lung is, that human lung possesses much fewer Clara (Club) cells than the rat and mouse lung and rat still much less than the mouse lung. Thus, risk for chemically induced toxicity and carcinogenicity mediated by and originating from Clara cells may rightfully be expected to be much lower in humans than in rats and these, in turn, to be much lower than in mice (Green 2000).

Various other CYP catalytic activities Beside the studies presented in the above chapter on styrene metabolism, quite early studies already led to the recognition that also with respect to other xenobiotic compounds their metabolism in the human lung was associated with organ selective toxicities as well as species differences in susceptibility.

Thus, Warren et al. (1982) recognized that the selective toxicity of naphthalene for the lung was associated by its CYP-catalyzed metabolism.

On the other end, Dowsley et al. (1999) reported that human lung microsomes metabolized 1,1-dichloroethylene to a reactive epoxide, but much slower than liver microsomes.

With respect to species differences Csanády et al. (1992) reported that butadiene was converted by human and rat lung microsomes to the monoepoxide at a more than 10-fold lower rate than by mouse lung microsomes, possibly providing an important contribution to the high susceptibility of the mouse for butadiene-induced lung carcinogenicity compared with the much more resistant rat.

Further examples are discussed below.

Several pieces of information are available (in addition to those presented in the two preceding chapters) concerning metabolism of xenobiotic compounds in the human lung by individual forms of CYP.

Chae et al. (1993) reported that in human lung CYP1A1 was predominantly responsible for the biotransformation of the carcinogen 6-nitrochrysene. Later on they determined that compared with human liver microsomes the rates of biotransformation of mono-nitropyrenes were 10- to 70-fold lower in human lung microsomes (Chae et al. 1999).

In the study by Yilmaz et al. (2019) the only substrate which showed extremely little, but clearly observable conversion by human lung slices was the CYP1A1 substrate AFQ056 (less than $0.1 \%$ conversion in $4 \mathrm{~h}$, estimated from the figure in Yilmaz et al. 2019), while no CYP3A4 activity toward midazolam and no activity toward the CYP2C substrates amodiaquine, diclofenac and diazepam was detected [quite in contrast to rat precision-cut lung slices, where they detected low, but clearly quantifiable CYP3A4 activity toward midazolam (about $0.3 \%$ substrate conversion in $4 \mathrm{~h}$ ) and toward the CYP2C substrates amodiaquine (about $0.6 \%$ conversion), diclofenac (about $0.4 \%$ conversion) and 
diazepam (about $2 \%$ conversion) and, most notably, about $30 \%$ conversion of the CYP1A1 substrate AFQ056].

While it is generally assumed that pneumonal CYP1A1 activity contributes to lung damage presumably via uncoupling-produced $\mathrm{H}_{2} \mathrm{O}_{2}$ (e.g. Mansour et al. 1988; Hazinski et al. 1989). Veith and Moorthy (2018) reported induction, inhibition and genetic knock-out data which rather suggest that CYP1A activity protects against hyperoxic lung injury, while their studies suggest that CYP1B1 activity indeed increases hyperoxic lung injury.

CYP2A6 which is clearly expressed at the mRNA level, but controversially at the protein level in the human lung (see above), plays an important role in the toxication of the tobacco-specific carcinogen NNK (4-methylnitrosamino1,3-pyridyl-1-butanone). However, the 7-hydroxylation of coumarin, which is characteristic for CYP2A6, has not been detected in human lung (Zhang et al. 2006). On the other hand, the closely related CYP2A13, which in human lung is very highly expressed at the mRNA level (see above), was found to have a catalytic efficiency for NNK much greater than any other CYP investigated previously (Su et al. 2000). Conversely, Brown et al. (2007) concluded from their study that neither CYP2A6 nor CYP2A13 levels appeared to constitute a predominant factor for NNK bioactivation in peripheral lung, at least for most subjects [in addition to CYPs, lipoxygenases and lipid hydroperoxides have been suggested to also be involved in NNK activation (Smith et al. 1995)]. Another tobacco-specific carcinogen, NNN ( $N^{\prime}$-nitrosonornicotine), is very efficiently activated by human CYP2A13 ( $\mathrm{Su}$ et al. 2000). Hydrogen bonding between the amino acid Asn297 and NNK appears important for substrate orientation and site-directed mutation of this residue to Ala strongly decreased the catalytic efficiency for NNK and NNN metabolism by 4- to 5 -fold (Schlicht et al. 2009). In addition, heterologously expressed CYP2A13 metabolizes $\mathrm{N}, \mathrm{N}$-dimethylaniline, $\mathrm{N}$-nitrosodiethylamine (NDEA), $\mathrm{N}$-nitrosomethylphenylamine, 2,6-dichlorobenzonitrile, hexamethylphosphoramide (HMPA), and 2'-methoxyacetophenone (Ding and Kaminsky 2003).

Human lung CYP2E1 activated vinyl carbamate, the intermediate in the pathway of the pneumotoxin and lung carcinogen ethyl carbamate to vinyl carbamate epoxide (Forkert et al. 2001).

The lung-selective human CYP2F1, recombinantly expressed, was shown to efficiently toxify several lung toxins including 3-methylindole (toxified by CYP2F-mediated dehydrogenation to an electrophilic methylene imine), naphthalene (toxified by epoxidation) (Lanza et al. 1999) and styrene (Nakajima et al. 1994). Human lung microsomes dehydrogenated 3-methylindole and this reaction was inhibited by the CYP2F inhibitor 5-phenyl-1-pentyne (Bernauer et al. 2009b). The human CYP2F1-catalyzed naphthalene turnover rate $(0.035 \mathrm{nmol} / \mathrm{min} / \mathrm{nmol} \mathrm{CYP})$ was more than 1000-fold lower than that of the mouse ortholog CYP2F2. Metabolic activation of the pneumotoxin 4-ipomeanol by the human CYP2F1 was rather low (Czerwinski et al. 1991), while the efficiency of the human CYP2F1 toward 3-methylindole was relatively high $\left(V_{\max } 1.3 \mathrm{nmol} / \mathrm{min} / \mathrm{nmol} \mathrm{CYP}\right.$, $K_{\mathrm{m}} 60 \mu \mathrm{M}$ ) (Buckpitt et al. 2002). Recombinant CYP2F1 metabolized benzene with a turnover rate of $0.01 \mathrm{pmol} / \mathrm{min} /$ pmol CYP and reasonably efficient $\left(V_{\max } / K_{\mathrm{m}} 2.6\right)$ (Sheets et al. 2004). The recombinant CYP2F1 also metabolized 7-ethoxycoumarin, 7-propoxycoumarin and 7-pentoxyresorufin, but not 7-ethoxyresorufin (Nhamburo et al. 1990).

The novel CYP2S1 metabolizes naphthalene (Karlgren et al. 2005). Since CYP2S1 occurs in the human lung it has been hypothesized that it contributes to naphthalene's pneumotoxicity (Buckpitt et al. 2002). Bui and Hankinson (2009) demonstrated that in presence of an oxidizing agent such as hydrogen peroxide human CYP2S1 metabolizes BP 7,8-dihydrodiol with a high rate to the ultimate carcinogens BP-7,8,9,10-dihydrodiol-epoxides.

Kelly et al. (1997) reported that aflatoxin $B_{1}$ was biotransformed in the human lung and that CYP3A played a role in this, but that this activity was very low compared with the same activity in the human liver. Van Vleet et al. (2001) observed that aflatoxin $\mathrm{B}_{1}$ was metabolized to an epoxide in NHBE (normal human bronchial epithelial cells). CYP2A13 was found to be chiefly responsible for the metabolism of aflatoxin B1 to its 8,9-epoxide in the human lung (He et al. 2006).

The toxication of benzo[a]pyrene 7,8-dihydrodiol to the ultimate mutagen/carcinogen 7,8-dihydrodiol 9,10-epoxide was catalysed by human lung microsomes and this biotransformation step was enhanced by the CYP3A activator $\alpha$-naphthoflavone (Shimada et al. 1989, 1992).

CYP3As are important for the metabolism of several inhaled drugs such as theophylline, salmeterol and glucocorticoids (Pavek and Dvorak 2008).

\section{Non-CYP oxidoreductases (Tables 2, 9)}

\section{Flavin-dependent monooxygenases (FMO)}

FMO transcripts FMO4 mRNA is expressed broadly in many human tissues, including the lung, while FMO2 mRNA is selectively expressed in human lung (Zhang and Cashman 2006).

Woenckhaus et al. (2006) observed in smokers bronchial and alveolar epithelium downregulation of FMO3 transcripts.

Nishimura and Naito (2006) observed in the human lung relatively high levels of FMO2, modest levels of FMO5 and low levels of FMO1, FMO3 and FMO4 mRNA. 
Leclerc et al. (2011) observed in the human bronchial mucosa high levels of FMO2 and moderate levels of FMO5 transcripts, and in pulmonary parenchyma high levels of both of them.

Zuo et al. (2018) demonstrated in samples-gained by bronchoscopy and by scraping from the human small airways epithelial Clara (Club) cells-transcripts of FMO2.

Table 9 Genes exhibiting high interindividual variability (CV $>10 \%)$ in primary cultures of HBEC. Reproduced from Courcot et al. (2012), with permission of the publisher

\begin{tabular}{|c|c|c|c|}
\hline Genes & Minimum $\beta C t$ & Maximum $\beta C t$ & $\begin{array}{l}\text { Coefficient } \\
\text { of variation }\end{array}$ \\
\hline \multicolumn{4}{|c|}{ Phase I enzymes } \\
\hline$C Y P 4 B 1$ & 17.09 & 23.85 & 15.41 \\
\hline$C Y P 2 W 1$ & 20.14 & 28.09 & 14.46 \\
\hline CYP11A1 & 22.04 & 28.91 & 13.83 \\
\hline CYP4A11/22 & 22.07 & 30.53 & 13.78 \\
\hline$A L D H 3 B 2$ & 17.29 & 23.01 & 12.17 \\
\hline DHRS2 & 21.97 & 28.71 & 12.13 \\
\hline$A L D H 1 A 2$ & 22.07 & 28.09 & 11.61 \\
\hline$A L D H 1 A 3$ & 10.41 & 13.85 & 11.61 \\
\hline$C Y P 26 B 1$ & 18.49 & 24.55 & 11.49 \\
\hline$A L D H 3 A 1$ & 15.75 & 19.96 & 11.16 \\
\hline FMO3 & 23.29 & 28.91 & 10.88 \\
\hline CYP4F11 & 15.04 & 19.14 & 10.38 \\
\hline$A D H 7$ & 16.31 & 20.72 & 10.27 \\
\hline \multicolumn{4}{|c|}{ Phase II enzymes } \\
\hline GSTT1 & 17.34 & 30.53 & ${\underline{28.58^{\underline{a}}}}^{\underline{a}}$ \\
\hline GSTM1 & 20.80 & 30.53 & $\underline{19.82^{\underline{a}}}$ \\
\hline$I N M T$ & 21.62 & 28.91 & 15.11 \\
\hline UGT1A10 & 22.52 & 30.53 & 14.66 \\
\hline$G S T T 2 / 2 B$ & 17.48 & 23.78 & 13.44 \\
\hline UGT1A8 & 23.10 & 30.53 & 12.28 \\
\hline$S U L T 2 B 1$ & 13.03 & 16.93 & $\underline{10.89}$ \\
\hline \multicolumn{4}{|l|}{ Transporters } \\
\hline$A B C A 4$ & 20.08 & 28.09 & 15.02 \\
\hline SLC7A5 & 11.68 & 14.83 & 13.06 \\
\hline SLCO4A1 & 16.41 & 21.86 & 12.85 \\
\hline SLC7A11 & 13.14 & 17.04 & 12.81 \\
\hline ABCC6 & 22.98 & 28.91 & 10.75 \\
\hline$A B C B 11$ & 22.33 & 27.98 & 10.70 \\
\hline$S L C O 5 A 1$ & 22.71 & 28.91 & 10.52 \\
\hline \multicolumn{4}{|c|}{ Nuclear receptors and other genes } \\
\hline$A R N T 2$ & 19.93 & 26.64 & 14.69 \\
\hline FOXA2 & 17.29 & 22.57 & 13.65 \\
\hline$H I F 3 A$ & 21.25 & 28.71 & 12.34 \\
\hline$M T 1 X$ & 12.01 & 15.71 & 12.61 \\
\hline
\end{tabular}

Interindividual variability in gene expression evaluated by calculation of the coefficient of variation $(\mathrm{CV}=\beta \mathrm{Ct} \mathrm{SD} /$ mean $\beta \mathrm{Ct})$

${ }^{a}$ Highest coefficients of variation underlined. Underlined genes represent genes that exhibited the highest coefficients of variation
FMO1 gene expression in the human bronchial airway epithelium was upregulated by acute and by chronic cigarette smoking. Likewise, in cultured human bronchial epithelial cells, which were obtained from non-smoking donors, FMO1 gene expression was upregulated by acute exposure to cigarette smoke (Billatos et al. 2018).

FMO catalytic activities FMO2, which is selectively expressed in the lung, differs from other FMOs in that it is capable to catalyse the $\mathrm{N}$-oxidation of certain primary alkyl amines (Dolphin et al. 1998; Chung et al. 2000). FMO2 catalytic activities include the $S$-oxygenation of low molecular mass thioureas such as the lung toxicant ethylene thiourea (Zhang et al. 2006).

Yilmaz et al. (2019) observed in human precision-cut lung slices about $0.7 \%$ conversion of the FMO substrate benzydamine in $4 \mathrm{~h}$ (while in rat precision-cut lung slices about $80 \%$ of the substrate were converted by FMO). These activities catalyzed by FMO are much higher compared with the (very much lower!) CYP activities in human (and rat) lung slices observed in the same study (Yilmaz et al. 2019) suggesting that FMO activities may quite generally be considerably more important for xenobiotic oxidation reactions in the lung than CYP activities, albeit preferentially toward structurally different groups of compounds. FMOs preferentially catalyze oxidations on soft nucleophilic centers, such as nitrogen and sulphur atoms, while CYPs preferentially catalyze oxidations on hard nucleophilic centers, such as carbon atoms (for a more complete discussion see OeschBartlomowicz and Oesch 2007).

\section{Alcohol dehydrogenase (ADH)}

Transcripts Nishimura and Naito (2006) observed in the human lung relatively high levels of ADH5 mRNA, modest levels of ADH1C, low levels of ADH1B, ADH4 and ADH7 and very low levels of ADH1B and ADH6, while levels of ADH1A were below quantification.

Woenckhaus et al. (2006) observed in smokers bronchial epithelium and alveolar epithelium downregulation of ADH1B (class I) transcripts.

Leclerc et al. (2011) found in healthy human bronchial mucosa ADH1B and ADH7 transcripts, both of them highly expressed, in pulmonary parenchyma ADH1B highly expressed, ADH7 at a very low expression level.

Van Dyck et al. (2014) reported the presence of ADH1A, ADH1C and ADH7 mRNA in human bronchial tissue, the decrease of $\mathrm{ADH} 1 \mathrm{~A}$ and $1 \mathrm{C}$ in smokers with lung or head and neck cancer compared with non-smokers and the decrease of all three of them in smokers with lung or head and neck cancer compared with smokers without cancer. 


\section{Aldehyde dehydrogenase (ALDH)}

Transcripts Nishimura and Naito (2006) observed in the human lung relatively high levels of ALDH2, modest levels of ALDH1A1, 1A2, 1A3, 1B1, 3A1, 3A2, 3B1, 5A1, 6A1, 7A1, 9A1, low levels of ALDH 4A1, 8A1 and very low levels of ALDH3B2 mRNA.

Leclerc et al. (2011) found in healthy human bronchial mucosa ALDH1A1, 1A3, 2, 3A1, 3A2, 3B1, 9A1 and 18A1 transcripts, all of them highly expressed. All of them were also present in pulmonary parenchyma, but several of them at a considerably lower expression level.

Van Dyck et al. (2014) reported the presence of ALDH3A1mRNA in human bronchial tissue, its increase in smokers without cancer compared with non-smokers, and its decrease in smokers with lung or head and neck cancer compared with smokers without cancer.

Zuo et al. (2018) demonstrated in samples-gained by bronchoscopy and by scraping from the human small airways epithelial Clara (Club) cells-transcripts of the ALDH1A1 as well as ALDH2 (the latter: mitochondrial).

$A L D H 3 A 1$ gene expression in the human bronchial airway epithelium was upregulated by acute and by chronic cigarette smoking. In cultured human bronchial epithelial cells, which were obtained from non-smoking donors, ALDH3AI gene expression was upregulated upon acute exposure to cigarette smoke (Billatos et al. 2018).

Takahashi et al. (2018) found that ALDH3A1 transcripts were overexpressed in bronchial brushings from currently smoking asthmatics compared with ex-smoking and nonsmoking asthmatics and non-smoking healthy individuals.

Líbalová et al. (2014) reported strong upregulation of $A L D H 1 A 3$ and $A L D H 3 A 1$ gene expression upon exposure of A549 cells to the extractable organic matters (EOMs) from the respirable fraction of airborne PM2.5 particles $(<2.5 \mu \mathrm{m})$ from three heavily polluted areas of the Czech Republic.

\section{NAD(P)H:quinone oxidoreductase (NQO)}

Nishimura and Naito (2006) observed in the human lung relatively high levels of NQO2 and modest levels of NQO1 mRNA.

Leclerc et al. (2011) observed in the human bronchial mucosa high levels of NQO1 transcripts and in pulmonary parenchyma moderate levels of FMO5 transcripts.

Takahashi et al. (2018) found that NQO1 transcripts were highly upregulated in bronchial brushings from currently smoking asthmatics compared with ex-smoking and nonsmoking asthmatics and non-smoking healthy individuals.
Aldehyde oxidase (AO)

Yilmaz et al. (2019) observed in human precision-cut lung slices $\mathrm{AO}$ activity toward carbazeran (about $0.2 \%$ substrate conversion in $4 \mathrm{~h}$ ) (while in rat precision-cut lung slices about $0.9 \%$ of the substrate was converted by $\mathrm{AO}$ in $4 \mathrm{~h}$ ).

\section{Aldo-keto reductases}

The aldo-keto reductase 1C1 (AKR1C1) (20 $\alpha$-hydroxysteroid dehydrogenase; dihydrodiol dehydrogenase) mRNA expression is highest in the lung, higher than in the liver and in four further investigated organs (El-Kabbani et al. 2011).

Leclerc et al. (2011) found in healthy human bronchial mucosa and in pulmonary parenchyma, respectively, the following AKR transcripts at the following expression levels (median DCt):

AKR1A1 14.9 15.1

AKR1B1 15.915 .2

AKR1B10 20.6 26.5

AKR1C1/2 16.2 17.7

AKR1C3 16.3 17.1

AKR6A5 17.4 15.8.

Miller et al. (2012) observed strong AKR1C immunoreactivity in human bronchial epithelium, but not in human alveolar pneumocytes.

Kalabus et al. (2012) showed in human lung tissue samples that carbonyl reductase 1 (CBR1) mRNA and protein were increased 1.5 -fold in smokers compared with neversmokers. Regarding these results, it is of interest to note that polynuclear quinones as well as ortho-quinones derived from polycyclic aromatic hydrocarbons such as BP are good substrates of CBR1 (Wermuth et al. 1986).

Lin et al. (2013) reported a marked decrease in AKR1C3 mRNA and protein in glucose-6-phosphate dehydrogenase knockdown human lung A549 cells.

Van Dyck et al. (2014) reported the presence of AKR1B10, 1C2, 1C3 and 1C4 mRNA in human bronchial tissue, the increase of all of them in smokers without cancer compared with non-smokers, the increase of AKR 1B10, $1 C 2$ and 1C3 mRNA in smokers with lung or head and neck cancer compared with non-smokers and the decrease of all four of them in smokers with lung or head and neck cancer compared with smokers without cancer. In the same study they reported the presence of DHRS9 (SDR family dehydrogenase/reductase 9) mRNA in human bronchial tissue and its increase in smokers without cancer compared with nonsmokers and in smokers with lung or head and neck cancer compared with non-smokers.

Zuo et al. (2018) demonstrated in samples—gained by bronchoscopy and by scraping from the human small airways epithelial Clara (Club) cells-transcripts of DHRS3 
(SDR family dehydrogenase/reductase 3) and DHRS9 (SDR family dehydrogenase/reductase 9).

Takahashi et al. (2018) found that AKR1C1 transcripts were more highly expressed in bronchial brushings from currently smoking asthmatics compared with ex-smoking and non-smoking asthmatics and non-smoking healthy individuals.

\section{Hydrolases}

\section{Epoxide hydrolase (EH) (Table 5)}

EHs have exogenous and endogenous functions, with respect to the topic of this review, xenobiotic metabolism, detoxifying and toxifying depending on and predictable from the structure of the xenobiotic compound in question (Bentley et al. 1977).

\section{Microsomal epoxide hydrolase (EPHX1 or mEH} or EH1) Transcripts Nishimura and Naito (2006) observed in the human lung modest levels of EPHX1 mRNA.

Somers et al. (2007) reported the presence of EPHX1 transcripts in the human lung parenchyma.

Leclerc et al. (2011) found in human bronchial mucosa and in pulmonary parenchyma high levels of EPHX1 transcripts.

Lin et al. (2013) reported a marked decrease in EPHX1 mRNA (and protein) in glucose-6-phosphate dehydrogenase knockdown human lung A549 cells.

Protein Amount of EPHX1 protein in human lung varied 6-fold between 92 individuals (Bernauer et al. 2009a).

Lin et al. (2013) reported a marked decrease in EPHX1 protein (and mRNA) in glucose-6-phosphate dehydrogenase knockdown human lung A549 cells and a marked increase in the sensitivity to the cytotoxicity of aflatoxin B1. The authors conclude that glucose-6-phosphate dehydrogenase is protective against the cytotoxicity of aflatoxin B1 by leading to a higher level of EPHX1 which, in turn, detoxifies the aflatoxin B1 derived 8,9-exo-epoxide more efficiently. They speculate that this may be caused by glucose-6-phosphate dehydrogenase providing increased levels of NADPH for NADPH oxidase to induce EPHX1.

Catalytic activities (Table 5) Very early on Lorenz et al. (1979) already observed in human lung considerable activities of microsomal epoxide hydrolase toward several substrates, statistically not different between cancer patients and non-cancer patients (Table 5), low in lung compared with liver. EPHX1 activity in human lung peripheral squamous cell carcinomas was considerably lower (only one third) compared with non-diseased portions of the lungs of the same subjects (Oesch et al. 1980) [which is in striking contrast to the rat liver, where EPHX1 was considerably increased in lung tumor tissue and has been identified as a preneoplastic antigen (Levin et al. 1978)].

Pacifici et al. (1988b) confirmed in the human lung microsomal fraction EPHX1 activity using styrene 7,8oxide as substrate. Using BP 4,5-oxide as substrate Petruzzelli et al. (1988) observed modest $(0.25 \pm 0.10 \mathrm{nmol} /$ $\mathrm{min} / \mathrm{mg}$ protein) EPXH1 activity in human pulmonary macrophages.

Omiecinski et al. (1993) observed a high correlation between human individual EPHX1 activity in blood lymphocytes and in their lungs and Omiecinski et al. (1994) reported a EPHX1 activity of $16 \mathrm{pmol} / \mathrm{mg} 9000 \mathrm{~g}$ supernatant protein/min already in the human fetus at a gestational age of 12 weeks with practically no change until birth and already approaching adult levels.

Boogaard and Bond (1996) observed in human lung microsomes considerable EPHX1 activity toward diepoxybutane, the putative ultimate genotoxin formed from butadiene. The activity in the human lung microsomes was much higher than in those of rodents, which are susceptible to the carcinogenic action of butadiene. In the rat lung microsomes the EPHX1 activity was, in turn, higher than in the lung microsomes of the mouse, the species most susceptible to the lung carcinogenic action of butadiene (see Table 5).

Li et al. (2011a) observed in human pulmonary microsomes $\mathrm{mEH}$ activity toward ethylene oxide as substrate (but not in pulmonary microsomes of rats or mice).

Soluble epoxide hydrolase (EPHX2 or sEH or CEH or EH2) Nishimura and Naito (2006) observed in the human lung modest levels of EPHX2 mRNA.

Somers et al. (2007) reported the presence of EPHX2 transcripts in the human lung parenchyma.

Pacifici et al. (1988a) observed in the human lung cytosolic fraction EPHX2 activity using trans-stilbene oxide as substrate. Strikingly, the specific activities were higher in the fetal lungs compared with adults $(43.2 \pm 19.2$ versus $8.5 \pm 2.8 \mathrm{pmol} / \mathrm{min} / \mathrm{mg}$ cytosolic protein) with very little changes during fetal development (determined at gestational ages between 17 and 23 weeks).

\section{Esterases/Amidases}

Nishimura and Naito (2006) observed in the human lung modest levels of carboxyl esterase (CES) CES1, CES2 and esterase D (ESD) mRNA.

Somers et al. (2007) reported the presence of CES1 transcripts in the human lung parenchyma with expression levels similar to those in the liver.

Leclerc et al. (2011) found in human bronchial mucosa and in pulmonary parenchyma high levels of CES 1 transcripts. 
Vickers et al. (1997) demonstrated in human lung slices efficient cleavage of the methylcarbonate cyclosporine A derivative SDZ SCP 764 liberating the esterase product $\mathrm{N}$-hydroxycyclosporin A five times faster than the $\mathrm{N}$-hydroxylation of cyclosporine A proceeded. The authors conclude that the lung plays an important role in the metabolism of this cyclosporin A derivative and will play an even greater role, when the compound is administered by inhalation.

Quite a number of prodrug esters are cleaved by human lung esterases to the active drug [e.g. ciclesonide (Nave et al. 2006)], or to more active derivatives [e.g. cleavage of beclomethasone dipropionate to the monopropionate (Würthwein and Rohdewald 1990)].

Esterase rates (with beclomethasone dipropionate as substrate) in the lung parenchymal cells were similar to those in human hepatocytes (Somers et al. 2007).

Microsomal carboxylesterase activities for the prototypical substrate $p$-nitrophenyl acetate varied from $19.02 \pm 2.28$ to $48.18 \pm 4.34 \mathrm{nmol} / \mathrm{mg}$ protein/min (Forkert et al. 2001). Using inhibitor and antibodies as diagnostic tools these authors found that in the human lung vinyl carbamate [the intermediate in the toxication pathway from ethyl carbamate to vinyl carbamate epoxide (Dahl et al. 1978)] was detoxified by carboxyl esterase $\mathrm{A}$.

Yilmaz et al. (2019) observed in human precision-cut lung slices esterase activity toward the CES1 substrate ramipril (about 7\% substrate conversion in $4 \mathrm{~h}$ ) (while in rat precision-cut lung slices between $10 \%$ and $20 \%$ of the substrate were hydrolyzed in $4 \mathrm{~h}$ ).

\section{Conjugating enzymes}

\section{Glutathione S-transferase (GST)}

GST transcripts Mainwaring et al. (1996) demonstrated the presence of GSTtheta transcripts in the human lung, especially in Clara (Club) cells. Levels were substantially lower than in the mouse lung.

Macé et al. (1998) observed GSTM1 transcripts in 25\% of human peripheral lung tissue and in $40 \%$ of bronchial mucosa and GSTM3 in 100\% of both of them. Tang et al. (2010) reported the presence of GSTM2 transcripts in the human lung. GSTP1 transcripts were observed by Thum et al. (2006) in human bronchial tissue and bronchial lavage.

Leclerc et al. (2011) found in healthy human bronchial mucosa and in pulmonary parenchyma, respectively, the following GST transcripts at the following expression levels (median DCt):

GSTA1 13.718 .7

GSTA2 14.320 .2

GSTA4 16.915 .9

GSTK1 15.215 .2

GSTO1 16.916 .0
GSTP1 10.812 .110 .6

MGST1 14.713 .8

MGST3 15.7 14.7.

Zuo et al. (2018) demonstrated in samples—gained by bronchoscopy and by scraping from the small airways epithelial Clara (Club) cells - transcripts of microsomal GST.

Van Dyck et al. (2014) reported the presence of GSTA1, A4, M1, M2, M3, M5, P1, T1,T2/T2B and MGST1 mRNA in human bronchial tissue, and their different levels in smokers without cancer compared with non-smokers, in smokers with lung or head and neck cancer compared with nonsmokers and in smokers with lung or head and neck cancer compared with smokers without cancer.

GST protein Howie et al. (1990) observed in human lung tissue predominantly GSTP $(0.53 \pm 0.04 \mathrm{mg} / \mathrm{g}$ cytosolic protein $)$, followed by GSTM $(0.07 \pm 0.02 \mathrm{mg} / \mathrm{g}$ cytosolic protein) and GSTA $B_{1}$ subunit $(0.07 \pm 0.01 \mathrm{mg} / \mathrm{g}$ cytosolic protein) and $B_{2}$ subunit $(0.04 \pm 0.01 \mathrm{mg} / \mathrm{g}$ cytosolic protein).

Anttila et al. (1993) reported the presence of the following GSTs in the human lung: GSTA1/A2, M1, M2, M3 and P1 in varying amounts: GSTA1/A2 and P1 most abundant; GSTM3 varying from abundant to minimal; GSTM2 minimal; GSTM1 ambiguous. GSTA1/A2 and P1 were found in the bronchial and bronchiolar epithelia, GSTM2 in the epithelia of the terminal airways, GSTM3 in ciliated airway epithelia and in lung smooth muscle cells. In the bronchial epithelia of current smokers GSTM3 was more abundant than in those of ex-smokers.

Wang et al. (2003) found GSTP1 as the most abundant form in the human lung.

Mainwaring et al. (1996) demonstrated the presence of GSTtheta protein in the human lung, especially in Clara cells. Levels were substantially lower than in the mouse lung.

Development GSTP1 protein was the major GST isoenzyme expressed in the developing human lung, being present at relatively high levels in the ductular columnar epithelium already at 12-18 weeks, then decreased upon differentiation, between gestational age of 24-27 weeks becoming largely negative in the distal airways, but still detectable in the proximal airways. GSTA1 and A2 proteins were expressed at low levels in the same locations (Cossar et al. 1990). Similar observations regarding GSTP were reported by Beckett et al. (1990) noticing a slight decrease (from 14.1 to 3.8 pmol per mg cytosolic protein) between late fetal to 2 weeks postnatal to 2 years adulthood. Concerning GSTM they observed a slight decrease (from 3.4 to $1.2 \mathrm{pmol} / \mathrm{mg}$ cytosolic protein), while GSTA1 and A2 remained relatively constant.

GST activity (Table 6) Very early on Lorenz et al. (1979) already observed considerable activities of GST in human 
lung, statistically not different between cancer patients and non-cancer patients (Table 6). Toward the broad-spectrum substrate CDNB the specific activities of human and rat lung microsomes were practically identical (Oesch et al. 1980).

Autrup et al. (1980) showed in cultured human tracheobrochial tissue a high proportion of BP metabolized to glutathione metabolites, much more than to glucuronides.

Pacifici et al. (1988b) observed in the human lung $9000 \mathrm{~g}$ supernatant GST activity using BP 4,5-oxide as substrate. Also using BP 4,5-oxide as substrate Petruzzelli et al. (1988) observed modest $(0.22 \pm 0.12 \mathrm{nmol} / \mathrm{min} / \mathrm{mg}$ protein $)$ GST activity in human pulmonary macrophages.

Boogaard et al. (1996) observed in human lung cytosol GST activity toward butadiene epoxide (a reactive metabolite of the rodent carcinogen butadiene) $(6.4 \pm 1.9 \mathrm{nmol} / \mathrm{min} /$ $\mathrm{mg}$ cytosolic protein), considerably less than in the mouse and rat lung cytosol $(162 \pm 16$ and $186 \pm 37 \mathrm{nmol} / \mathrm{min} / \mathrm{mg}$ cytosolic protein, respectively).

Li et al. (2011a) observed in human pulmonary cytosol GST activity toward ethylene oxide as substrate.

GST specific activity toward the broad-spectrum substrate CDNB was markedly lower in the human lung compared with the human liver (Castell et al. 2005).

\section{UDP-glucuronosyltransferase (UGT)}

As opposed to the human liver, expression of UGTs on the mRNA level, protein level and enzymatic activity level isgenerally speaking—quite low in the human lung.

UGT transcripts Turgeon et al. (2001) reported the presence of the following UGT mRNAs in the human lung: UGT2B4, 2B7, 2B10, 2B11, 2B15 and 2B17.

Beclomethasone dipropionate increased the expression of UGT2B4 and UGT2B11 transcripts in cultured bronchial epithelial cells, which was hypothesized to possibly be related to individual differences in response to treatment with steroids (Kuzuya et al. 2004).

Somers et al. (2007) reported the presence of UGT1A1, 1A4, 1A6-1, 1A6-2, 2A1 2B4, 2B7 and 2B11 transcripts in the human lung parenchyma, but all of them at low expression levels, except UGT2A1, the expression level of which was higher in the lung than in the liver. Using PT-PCR Nakamura et al. (2008a, b) did neither detect UGT transcripts of the UGT1A subfamily nor of the UGT2B subfamily. Using RT-PCR Courcot et al. (2012) detected only traces of UGT1A1, 1A4, 1A5, 1A7, 1A8, 1A9 and 1A10 and only low expression of UTG1A3 and 1A6, while UGT2A1, 2A2, 2A3, 2B 4, 2B7, 2B10, 2B11, 2B15, 2B17 and 2B28 were not detected. Courcot et al. (2012) reported the presence of the following UGT transcripts in the human lung: UGT1A1 (trace), 1A3, 1A6, 1A10 (trace), while transcripts of the following UGTs were not detected: UGT1A4, 1A5,
1A7, 1A8, 1A9, 2A1, 2A2, 2A3, 2B4, 2B7, 2B10. 2B11, 2B15, 2B17, 2B28.

Van Dyck et al. (2014) reported the presence of UGT1A6, 1A9 and 2A1 mRNA in human bronchial tissue, and their different levels in smokers without cancer compared with non-smokers and in smokers with lung or head and neck cancer compared with non-smokers.

UGT catalytic activities (Table 7) UGT activities in the human lung are low. Their contribution to the total xenobiotic glucuronidation in the human organism is, therefore, relatively modest (Zhang et al. 2006). Only trace activities (with 1-naphthol as substrate) were observed in lung parenchymal cells by Somers et al. (2007). Pacifici et al. (1988b) observed in the human lung microsomal fraction low UGT activity $(0.01 \mathrm{nmol} / \mathrm{min} / \mathrm{mg}$ microsomal protein) toward 1-naphthol as substrate. Zheng et al. (2002) observed in none of the human lung specimen's microsomes tested UGT activity toward 3-, 7- and 9-hydroxy-BP, although these activities were clearly seen in all upper aerodigestive tract specimen microsomes (tongue, tonsil, larynx, esophagus).

UGT2B7, the mRNA of which was demonstrated in human lung (see above), is catalyzing the glucuronidation of the lung carcinogen NNAL (4-(methylnitrosamino)-1-(3pyridyl)-1-butanol) (Ren et al. 2000).

Yilmaz et al. (2019) observed in human precision-cut lung slices very low UGT activity toward triclosan (about $0.1 \%$ conversion in $4 \mathrm{~h}$ ) as opposed to a remarkable activity in rat precision-cut lung slices (about $40 \%$ substrate conversion in $4 \mathrm{~h}$ ). About $0.2 \%$-methylumbelliferone were glucuronidated by the human lung slices as compared to $5 \%$ by the rat lung slices.

\section{Sulfotransferase (SULT)}

SULT transcripts Her et al. (1996) demonstrated in human fetal lung the presence of SULT1E1 mRNA, Sakakibara et al. (1998) the presence of SULT1C2 mRNA.

SULT2B1b mRNA (but no SULT2B1a mRNA) was found in human lung tissue by He et al. (2005), while Gamage et al. (2006) report that SULT1B1_v1 and SULT1B1_v2 mRNA are found in human lung tissue.

Somers et al. (2007) reported the presence of SULT1A1, $1 \mathrm{~A} 2,1 \mathrm{~A} 3 / 4,1 \mathrm{C} 2,2 \mathrm{~A} 1$ and $2 \mathrm{~B} 1 / 2$ transcripts in the human lung parenchyma at expression levels similar or higher than those in the liver [SULT1A2 mRNA may not be translated into a protein; discussed in Gamage et al. (2006)].

SULT1C4 is expressed at relatively high levels in the human foetal lung (Sakakibara et al. 1998).

SULT proteins Hume et al. (1996) reported that phenol sulfotransferase (SULT1A1/2/3) was present in the human fetus, being at its peak expression in the early foetus and 
at this point of development being widely distributed throughout the respiratory epithelium, with later development decreasing in its levels and becoming in its localization predominantly restricted to the more proximal airways. The fetal development of hydroxysteroid sulfotransferase (SULT1E1/2A1/2B1b) was quite different, being present in early gestation at very low levels, but then increasing rapidly and reaching its peak at 1 year postnatally. In the mature epithelium the localization of both, phenol sulfotransferase and hydroxysteroid sulfotransferase, was in ciliated cells and in non-ciliated secretory cells and basal cells, but not in mucus-secreting cells.

He et al. (2005) observed the presence of SULT2B1b protein (but no SULT2B1a protein) in human lung. The enzyme was localized to the cytoplasm of ciliated epithelial cells in "terminal bronchia" of the human lung.

SULT1A1 and $1 \mathrm{~A} 3$ proteins have been localized to the epithelia of the human lung bronchioles (Gamage et al. 2006).

SULT1C4 and SULT1E1 were observed at relatively high levels in the human fetal lung (Lindsay et al. 2008).

SULT catalytic activities (Table 7) Autrup et al. (1980) showed in cultured human tracheobrochial tissue a high proportion of BP metabolized to sulphates, much more than to glucuronides.

Pacifici et al. (1988b) observed in the human lung $9000 \mathrm{~g}$ supernatant SULT activity using 2-naphthol as substrate.

Since SULT2B1b, the presence of which on transcript and protein level was proven in the human lung (see above), is selective for $3 \beta$-hydroxysteroids such as cholesterol, pregnenolone and dehydroepiandrosterone, He et al. (2005) speculated that this enzyme may be essential for the regulation of local steroid metabolism in the human lung.

Somers et al. (2007) reported that sulfotransferase rates (with 1-naphthol as substrate) in the lung parenchymal cells were similar to those in human hepatocytes.

Cappiello et al. (1991) observed in the human fetal lung (gestational age 18-25 weeks) higher activity of "dopamine SULT" ("TL", SULT1A3) than in the adult lung (22-76 years old individuals), while " $p$-nitrophenol SULT" ("TS", SULT1A1) in the fetal lung was about one third of that in the adult lung. However, Richard et al. (2001) reported that SULT1A1 was higher in the human fetal lung than in the postnatal lung, while they observed that SULT1A3 was higher in the fetal compared with the neonatal lung.

Yilmaz et al. (2019) observed in human precision-cut lung slices SULT activity toward triclosan (about $40 \%$ substrate conversion in $4 \mathrm{~h}$ ) (while in rat precision-cut lung slices only about $3 \%$ of the substrate was converted by SULT). About 7\% 4-methylumbelliferone were converted by the human lung slices SULT (as compared to about $20 \%$ by the rat lung slices).

\section{$\mathrm{N}$-Acetyltransferase (NAT)}

NAT transcripts Macé et al. (1998) observed NAT1 mRNA in $100 \%$ of the human peripheral lung tissue and of the bronchial mucosa, NAT2 mRNA in all peripheral lung tissues, but not in the bronchial mucosa.

NAT protein Dairou et al. (2009) observed in the human lung cell lines A549 and 16HBE NAT1 protein.

NAT activity (Table 7) Pacifici et al. (1986) observed in the adult human lung 150,000 g supernatant considerable activity of NAT for PABA (para-aminobenzoic acid) as substrate, one third of the specific activity observed in the liver. Surprisingly high activities were found in the human foetal lung, almost identical to the adult lung.

Dairou et al. (2009) reported for the following substrates in the human lung cell line A549 and 16HBE cytosols NAT activities: benzidine, $\beta$-naphthylamine, 4 -aminobiphenyl, $p$-aminosalicylic acid and 2-aminofluorene. Exposure of the cells to pathophysiologically relevant levels of $\mathrm{H}_{2} \mathrm{O}_{2}$ or peroxynitrite led to impairment of these activities.

Yilmaz et al. (2019) observed in human precision-cut lung slices NAT activity toward $p$-toluidine (about 30\% substrate conversion in $4 \mathrm{~h}$ ) (in rat precision-cut lung slices about $50 \%$ of the substrate were converted).

\section{Xenobiotica-metabolizing enzymes in human lung derived cells and cell lines as well as reconstructed lung models}

One major advantage of unbroken cell systems compared with homogenates/microsomes for studies on xenobiotic metabolism is the presence of natural concentrations of cofactors in unbroken cells, which can have very profound consequences for xenobiotc metabolism and consequent events such as metabolism-dependent mutagenicity and other toxicities (Glatt et al. 1981).

\section{Cell lines}

Many mechanistic details are more quickly and relatively easily investigated in cell lines as opposed to organ homogenates. Compared with primary cells in culture, cell lines have the advantage of being a more homogeneous cell population, results are easier to reproduce, cell lines are easier to handle and are readily available. A main disadvantage of cell lines compared with primary cells is that lines do not retain 
many differentiated functions and are more distant from the in vivo situation than are primary cells.

The ease of handling lung-derived cell lines have led to the wide-spread use of animal lung-derived cell lines including genetically modified cell lines for mechanistic problem solving (e.g. Schmalix et al. 1995; Landsiedel et al. 2011). However, the present review focusses exclusively on human lung-derived cells and cell lines as well as reconstructed lung models.

An overview on the expression of xenobiotic metabolismrelated genes in various cell lines and primary cells derived from human lung tissue is presented in Table 8 .

Human lung cell lines have been established from lung cancer tissue or by viral transformation of normal human lung tissue with the aim to manifest major characteristics of either bronchial cells, Clara (Club) cells of the bronchioli or alveolar epithelial cells. Ramirez et al. (2004) achieved generation of an immortalized cell line from normal (noncancer) human bronchial epithelial cells without the use of an immortalizing virus/viral oncoprotein. They transfected hTERT (human telomerase reverse transcriptase) to prevent senescence and the cell cycle protein cdk (cyclin-dependent kinase) 4. This allowed for continuous replication and at the same time for differentiation when the cells were taken up to the air-liquid interphase (Vaughan et al. 2006). Although very elegant, the cell line has the draw-back of a limited life-span (Ramirez et al. 2004). Several attempts to establish human bronchial epithelial cell lines have successfully led to lines, however, with marked differences compared with their normal counterparts (Reddel et al. 1993; Mace et al. 1997; Ramirez et al. 2004).

Walters et al. (2013) developed a human airway epithelial basal cell line providing multiple differentiation potential.

Two very frequently (perhaps the most frequently) used human lung-derived cell lines for studies on human pulmonary xenobiotic metabolism are A549 and BEAS-2B. A549 consists of cells derived from human alveolar type II adenocarcinoma (Lieber et al. 1976) and has been characterized as a type II pulmonary epithelial cell model for xenobiotic metabolism (Foster et al. 1998). BEAS-2B is derived from normal human lung epithelial cells which were immortalised using an adenovirus 12-SV40 hybrid virus (Reddel et al. 1988). Also quite frequently used and quite well characterized are the lung adenocarcinoma NCI-H322 cells derived from bronchiolar Clara cells (Ueng et al. 2000; Adissu and Schuller 2004).

Courcot et al. (2012) showed in their broad study on xenobiotic metabolizing enzyme (and transporter)-related gene expressions (Table 8) (quite expectedly) that none of the lung models [i.e. none of the lung-derived ten cell lines and also none of the four lung-derived primary human bronchial epithelial cells (HBECs) considered] mirrors the situation in the fresh lung tissue samples perfectly. As generally seen in in vitro models (Olsavsky et al. 2007; Hart et al. 2010; Bourgine et al. 2012), the number of expressed genes was in all investigated models considerably lower than in the lung tissues. 57 of the 380 genes studied were not expressed in all of these models, 37 of these were also not expressed in the lung tissues. However, some trends, which may be helpful for choosing a relatively suitable model, became apparent (see Table 8): primary human bronchial epithelial cell cultures (HBECs) were with respect to their tissue of origin, the bronchial mucosa, much closer in their expression profile of xenobiotica-metabolizing enzyme genes than any of the cell lines. Between cell lines there were substantial differences. H292 and BEAS-2B cell lines showed the lowest number of dysregulated genes compared with human non-tumour lung tissue and, therefore, appear to present for xenobiotic metabolism studies a relatively acceptable substitute for the primary cells, which are clearly better in this respect, but are more difficult to handle. However, the following distortions should be kept in mind: CYP4B1 expression was observed only in primary HBECs, BEAS-2B and H292; ADH1B expression only in BEAS-2B; CES1 only in primary HBECs, A549, H460 and H727. Compared with the lung tissues expression of CYP1A1 and CYP1B1 was much higher ("unnaturally high") in most of the investigated models (CYP1B1 expression especially high in 16HBE and BEAS-2B), while conversely CYP2B6, CYP2F1 and CYP4B1 expression was much lower or nil in most of the investigated models. With respect to xenobiotica-metabolizing phase II enzymes, despite high gene expression of GSTA1/ GSTA2 and INMT1 in the bronchial epithelial mucosa and pulmonary parenchyma, expression was not detected in most lung models studied (see Table 8). The highest overall similarity of the gene expression pattern between the fresh bronchial mucosa was quite expectedly observed for the primary bronchial epithelium cells HBEC $(r=0.76)$ followed by the bronchial epithelial cell line BEAS-2B $(r=0.72)$. The highest similarity for the pulmonary parenchyma was observed for BEAS-2B $(r=0.76)$ followed by HBEC $(r=0.73)$. On the other end, the lowest similarity was observed between the bronchial mucosa compared with 16HBE and 1HAEO $(r=0.61)$ and between the pulmonary parenchyma compared with 16HBE $(r=0.65)$ and 1HAEO $(r=0.62)$. The frequently used A549 cell line derived from an adenocarcinoma was more similar with tumor tissue, especially adenocarcinoma tissue, than with healthy bronchial mucosa or pulmonary parenchyma. Considering the gene expression quantitatively (gene expression levels more than 4-fold different from the non-tumorous lung tissues) HBEC and BEAS-2B showed the lowest number of differently expressed genes compared with bronchial mucosa and $\mathrm{H} 292$ and BEAS-2B showed the lowest number of differently expressed genes compared with pulmonary parenchyma, while the L-132 cell line had the highest number of differently expressed 
genes (compared with both non-tumorous tissues). It should also be taken into account that the investigated expression of some genes was quite variable in the primary HBECs, especially GSTM1 and GSTT1 with a coefficient of variation of almost $20 \%$ and almost $30 \%$, respectively (Table 9).

Castell et al. (2005) investigated comparatively several xenobiotica-metabolizing enzyme activities in A549 cells versus human lung tissue. As is evident from the data presented in Table 10 as far as the investigated activities were present in human lung microsomes, they were present in the A549 cells as well. However, the relative activities were dramatically distorted. While the investigated phase II activities of GST and UGT were similar between lung and A549 cells, the phase I activities ECOD and testosterone $6 \beta$-hydoxylase were 6 - to 12 -fold different and
PROD and coumarin hydroxylase 270-387-fold different. Similarly, induction of xenobiotica-metabolizing enzyme activities takes place in A549 cells after treatment with some known inducers, but not after treatment with some others, which increases the distortion. Various types of xenobiotica-metabolizing enzymes' induction require the presence of different regulators, of which AhR, ARNT (and even AhR repressor) have been demonstrated to be present in A549 cells (Iwanari et al. 2002; Tsuchiya et al. 2003) as well as the glucocorticoid receptor (Hukkanen et al. 2003). However, CAR and PXR, which participate in the regulation of CYP2B6, 2C, 3A4 and 3A7 (Waxman 1999), were not detected in A549 cells (Hukkanen et al. 2003). As a consequence, the lung-selective CYP3A5 is induced in A549 cells by glucocorticoids such as dexamethasone, but not by rifampicin and other prototypical

Table 10 Xenobiotica-metabolizing enzyme activities in human lung and human lung cells in culture

\begin{tabular}{|c|c|c|c|c|c|c|}
\hline $\begin{array}{l}\text { Substrate (preferentially } \\
\text { for) }\end{array}$ & Lung & $\begin{array}{l}\text { Primary type } \\
\text { II pneumo- } \\
\text { cytes }\end{array}$ & Alveolar macrophages & A549 & $16 \mathrm{HBE}$ & References \\
\hline EROD (CYP1) & $<0.001^{\mathrm{a}}$ & bd & bd & $<0.001^{\mathrm{a}}$ & & $\begin{array}{l}\text { Castell et al. (2005), Dimova } \\
\text { et al. (2001) }\end{array}$ \\
\hline MROD (CYP1A2) & $<0.001^{\mathrm{a}}$ & & & $<0.001^{\mathrm{a}}$ & & Castell et al. (2005) \\
\hline PROD (CYP2B) & $0.351 \pm 0.416^{\mathrm{a}}$ & $8.8 \pm 0.8^{\mathrm{b}}$ & bd & $0.001 \pm 0.001^{\mathrm{a}}$ & & $\begin{array}{l}\text { Castell et al. (2005), Dimova } \\
\text { et al. (2001) }\end{array}$ \\
\hline BROD (CYP3A,2B,1A1) & & $27.2 \pm 1.5^{\mathrm{b}}$ & $0.74 \pm 0.20^{\mathrm{b}}$ & & & Dimova et al. (2001) \\
\hline $\begin{array}{l}\text { Coumarin- } \mathrm{OH}^{\mathrm{c}} \\
(\mathrm{CYP} \underline{2 \mathrm{~A}}, 1 \mathrm{~A}, 3 \mathrm{~A})\end{array}$ & $30.2 \pm 26.3^{\mathrm{a}}$ & & & $0.078 \pm 0.037^{\mathrm{a}}$ & & Castell et al. (2005) \\
\hline $\begin{array}{l}\text { Testosterone } 6 \beta-\mathrm{OH}^{\mathrm{c}} \\
(\mathrm{CYP} \underline{\mathrm{A}}, 1 \mathrm{~A})\end{array}$ & $0.135 \pm 0.033^{\mathrm{a}}$ & & & $0.023 \pm 0.021^{\mathrm{a}}$ & & Castell et al. (2005) \\
\hline $\begin{array}{l}\text { ECOD (CYP broad spec- } \\
\text { trum) }\end{array}$ & $1.91 \pm 0.81^{\mathrm{a}}$ & & & $0.165 \pm 0.061^{\mathrm{a}}$ & & Castell et al. (2005) \\
\hline DCPIP (NQO) & & $423 \pm 119^{e}$ & $72.6 \pm 50.2^{\mathrm{e}}$ & & & Dimova et al. (2001) \\
\hline $\begin{array}{l}\text { CDNB (GST broad spec- } \\
\text { trum) }\end{array}$ & $45.3 \pm 12.0^{\mathrm{d}}$ & $104 \pm 31^{\mathrm{e}}$ & $29.0 \pm 11.5^{\mathrm{e}}$ & $31.9 \pm 18.7^{\mathrm{d}}$ & & $\begin{array}{l}\text { Castell et al. (2005), Dimova } \\
\text { et al. (2001) }\end{array}$ \\
\hline $\begin{array}{l}\text { 4-Methylumbelliferone } \\
\text { (UGT) }\end{array}$ & $48.2 \pm 71.9^{\mathrm{a}}$ & & & $49.0 \pm 1.2^{\mathrm{a}}$ & & Castell et al. (2005) \\
\hline Benzidine (NAT) & & & & $0.94 \pm 0.32^{\mathrm{d}}$ & $1.39 \pm 0.21^{\mathrm{d}}$ & Dairou et al. (2009) \\
\hline$\beta$-Naphthylamine (NAT) & & & & $3.37 \pm 0.81^{\mathrm{d}}$ & $4.04 \pm 1.14^{\mathrm{d}}$ & Dairou et al. (2009) \\
\hline 4-Aminobiphenyl (NAT) & & & & $0.99 \pm 0.21^{\mathrm{d}}$ & $1.74 \pm 0.84^{\mathrm{d}}$ & Dairou et al. (2009) \\
\hline $\begin{array}{l}p \text {-Aminosalicylic acid } \\
\text { (NAT) }\end{array}$ & & & & $6.62 \pm 1.24^{\mathrm{d}}$ & $9.23 \pm 1.42^{\mathrm{d}}$ & Dairou et al. (2009) \\
\hline 2-Aminofluorene (NAT) & & & & $4.69 \pm 1.32^{\mathrm{d}}$ & $7.37 \pm 1.51^{\mathrm{d}}$ & Dairou et al. (2009) \\
\hline
\end{tabular}

Only constitutive activities; unless otherwise stated values either are mean \pm SD or there was no indication in the original literature

$B d$ below detection, BROD CDNB, 1-chloro-2,4-dinitrobenzene, $C Y P$ cytochrome P450, DCPIP 2,6-dichlorophenolindophenol, ECOD 7-ethoxycoumarin $O$-deethylase, $E R O D$ 7-ethoxyresorufin $O$-deethylase, $G S T$ glutathione $S$-transferase, $M R O D$ 7-methoxyresorufin $O$-dealkylase, $N A T$ $N$-acetyltransferase, $P R O D$ 7-pentoxy $O$-depentylase, $U G T$ UDP-glucuronosyl transferase

${ }^{\mathrm{a}} \mathrm{pmol} / \mathrm{min} / \mathrm{mg}$ microsomal protein

${ }^{\mathrm{b}} \mathrm{pmol} / \mathrm{min} / \mathrm{mg}$ cell homogenate

c_-OH = hydroxylation

${ }^{\mathrm{d}} \mathrm{nmol} / \mathrm{min} / \mathrm{mg}$ cytosolic protein

${ }^{\mathrm{e}} \mathrm{nmol} / \mathrm{min} / \mathrm{mg}$ cell homogenate 
CYP3A4 inducers (Hukkanen et al. 2003). Thus, irrespective of whether constitutive xenobiotica-metabolizing enzyme activities or their induction are important for a given study, the use of the probably most frequently used pulmonary cell line A549 needs caution, in particular if CYP1A2, 2A13 or 2E1 are in question, since they are not expressed in A549 cells (Castell et al. 2005). In the study by Hukkanen et al. (2000) quite a number of CYPs were in A549 cells even not detected on the mRNA level: CYP1A2, 2A6, 2A7, 2A13, 2F1, 3A4 and 4B1.

Dairou et al. (2009) observed in the human lung cell lines A549 and 16HBE the presence of NAT1 protein, an enzyme relatively little studied in the human lung.

\section{Some individual examples pertinent to mechanistic studies on xenobiotic metabolism/metabolizing enzymes in human lung cell lines}

Carr et al. (2003a) reported using the two cell lines, A549 and BEAS-2B, that the promoter region from -1681 to + 115 of the gene, which codes for the lung-selective CYP2F1, supports its transcriptional activity, but that this is not the case in the human liver (hepatoma)-derived cell line HepG2. In addition, they observed that in the lung cell line BEAS-2B and in human lung tissue the promoter region from -182 to -152 , which contains 2 E-box elements, interacts with nuclear proteins, but that this is not the case in material derived from liver or heart. All of this is quite well in line in these cell lines concerning the lung-selective expression of CYP2F1 in the native human lung.

The factors potentially responsible for the selective expression of CYP4B1 mRNA in the human lung were investigated by Poch et al. (2005) in cell lines derived from human lung versus such lines derived from human liver or kidney. They observed in all lines a positively acting element between positions -139 to -45 , but only in HepG2 a negatively acting element between positions -457 to - 216. Moreover they observed a lung-specific positively acting distal element between positions -1087 to -1008 in the lung-derived A549 and BEAS-2B, but not in the liverderived HepG2 nor in the kidney-derived 293 cells. Within this distal element they observed two sites to which specific nuclear proteins of the lung-derived A549, but not of the liver-derived HepG2, bound. In addition, they identified in the proximal region two Sp sites (between -118 and -114 , and -77 and - 73) that bound Sp1 and Sp3 and a synergistic $\mathrm{Sp} 1$-dependent interaction between this proximal and the distal regulatory element. Neither $\mathrm{Sp} 1$ nor $\mathrm{Sp} 3$ bound in human liver tissue to the CYP4B1 proximal region, but did so in the human lung tissue. The authors conclude that the selective expression of CYP4B1 mRNA in the human lung depends on the synergistic interaction between the proximal Sp binding sites with the distal regulatory element.
Biggs et al. (2007) reported that in the human lung cell line A549 a double E-box repressor is present in the 5'-upstream region of the CYP3A4, but not CYP3A5 promoter, which diminishes CYP3A4 expression. They showed by site-directed mutations that a $C A A T$-box and a basic transcriptional element are important for the preferential expression of CYP3A5 over CYP3A4 in this cell line and, hence, quite probably also in the human lung. The CYP3A4 promoter contains a 57-bp insertion fragment, the deletion of which increased CYP3A4 expression in A549 cells and also in NHBE (normal human bronchial epithelial cells) (but not in hepatocytes), while its insertion into the CYP3A5 promoter decreased the CYP3A5 expression in A549 cells and NHBE (but not in hepatocytes).

In the same cell line A549 the expression of CYP3A5 mRNA was induced by glucocorticoids, but not by the standard CYP3A4 inducers rifampicin, clotrimazole or nifedipine (Hukkanen et al. 2003; Raunio et al. 2004). In line with this, the studies also showed no expression of the transcription factors CAR and PXR in this human lung cell line (Hukkanen et al. 2003).

Xiang et al. (2015) investigated in the cell line A549 a possible coordinate transcriptional control of the phase I gene CYPA13, the phase II gene UGT2B17 and the phase III gene $A B C B 1$, all three known to be involved in the control of the nicotine-derived lung carcinogen NNK (4-(methylnitrosamino)-1-(3-pyridyl)-1-butanone. They found that the lung organogenesis transcription factor FOXA2 exerted a coordinate positive control on the transcriptional activation of these three genes. Genetic manipulation of the FOXA2 consistently led to a corresponding modulation of the binding of FOXA2 to the promotor regions of all three genes CYPA13, UGT2B17 and $A B C B 1$, followed by a corresponding change in the levels of their transcripts and proteins. The authors conclude that they have established FOXA2 as a core trans-acting modulator of the coordinate expression of these three genes.

Juricek et al. (2015) investigated the differential response of CYP1A1 transcript expression and consequent EROD activity in the human lung epithelial cell line NCI-H292 to three aromatic amines and their acetylated metabolites. Interestingly the response did not at all follow a unified pattern. The acetylated metabolite 2-acetylaminofluorene significantly induced CYP1A1 transcript and consequent EROD expression, while the parent aromatic amine 2 -aminofluorene did not. However, quite to the contrary the parent amine 2-naphthylamine efficiently enhanced CYP1A1 transcript and consequent EROD activity expression while its acetylated metabolite, 2-acetylaminonaphthalene was much less effective. Finally, neither the aromatic amine 4-aminobiphenyl nor its acetylated metabolite 4-acetylaminobiphenyl led to an increase in the CYP1A1 transcript nor to EROD activity expression. 
Kalabus et al. (2012) observed that incubation of the human lung cancer cell line A549 with $2.5 \mu \mathrm{M}$ BP led to a 3-fold and 1.5-fold increase of CBR1 (carbonyl reductase 1) mRNA and protein, respectively, and nuclear translocation of AhR and ARNT was increased. A XRE (xenobiotic response element) motif in the proximal promoter of the CBR1 gene mediated the induction. Regarding these results, it is of interest to note that polynuclear quinones as well as ortho-quinones derived from polycyclic aromatic hydrocarbons such as BP are good substrates of CBR1 (Wermuth et al. 1986).

Jiang et al. (2006) showed in human bronchoalveolar H358 cells (obtained from the American Type Culture Collection ATCC no. CRL-5807) that BP-7,8,-dione inducedvia AhR activation - the expression of the constitutively not present CYP1A1/1B1 transcript, protein and catalytic activity, leading to formation of the reactive genotoxin/carcinogen BP 7,8-dihydrodiol 9,10-epoxide. Since the BP-7,8,dione was formed from BP by AKR1A1 (aldo-ketoreductase 1A1), this established a functionally important cooperation between AKR1A1 and CYP1A1/1B1.

\section{Some examples of studies on xenobiotica-metabolizing enzyme modulations in human lung-derived cell lines}

Induction of CYP1A1 mRNA has been shown to occur and to be mediated by AhR and ARNT in many human pulmonary cell lines (Hukkanen et al. 2002).

Rivera et al. (2002) observed CYP2S1 mRNA induction by TCDD in the human lung epithelial cell line A549. The induction of CYP2S1 is the first example of a CYP induction by TCDD outside the CYP1 family.

Transforming growth factor $\beta 1$ represses induced and basal expression of CYP1A1 and CYP1B1 and basal expression of AhR in the cell line A549 cells (Hukkanen et al. 2002).

The widely discussed "red wine antioxidant" resveratrol inhibited the expression of CYP1A1 and CYP1B1 mRNA in BEP2D cells (Mollerup et al. 2001).

Palackal et al. (2002) showed that aldo-keto reductase 1C (AKR1C) transcripts, which are dramatically overexpressed in human small cell lung cancer, also are highly expressed in the human lung carcinoma cell line A549. A549 cell lysates effectively converted the 3,4-dihydrodiol metabolite of the potent carcinogen DMBA (7,12-dimethylbenz[a]anthracene) to the highly electrophilic metabolite $o$-quinone, which, in turn, was further metabolized to mono- and bis-thioether conjugates.

\section{Some examples of studies on the effect of airborne} environmental xenobiotics on human lung cell lines

Lepers et al. (2014) observed a very strong increase in CYP1A1 and CYP1B1 mRNA in BEAS-2B cells upon exposure to particulate matter (urban, industrial and also rural samples, as well as $\mathrm{PM}_{2.5}$ ). EROD activities were detected only after exposure of the cells to these particulate matters.

Líbalová et al. (2014) reported strong upregulation of $C Y P 1 A 1$ and $1 B 1$ gene expression upon exposure of human lung A549 cells to the extractable organic matters (EOMs) from the respirable fraction of airborne PM2.5 particles $(<2.5 \mu \mathrm{m})$ from three heavily polluted areas of the Czech Republic.

Libalova et al. (2016) demonstrated a considerable increase of CYP1A1 and 1B1 mRNA upon exposure of BEAS-2B cell line to diesel exhaust particles and Rynning et al. (2018) demonstrated increases of the same CYPs mRNAs in HBEC3 (human bronchial epithelial cell line) upon exposure to diesel exhaust particles.

Longhin et al. (2016) observed increases in the mRNA levels of AKR1C1, 1C2 and 1C3 upon exposure of the BEAS-2B human lung cancer cell line to urban particulate matter and Libalova et al. (2016) demonstrated in BEAS2B cells a considerable increase of AKR1C2, 1C3 and 1C4 mRNA upon treatment with diesel exhaust particles.

Courcot et al. (2012) compared the expression of CYP and further xenobiotic metabolizing enzymes (totally 380 genes) transcripts in four primary cell cultures of human bronchial epithelial cells and ten commonly utilized lung cell lines with human lung tissue. Primary cell cultures had the highest similarity in the expression of these genes with human bronchial mucosa. Of the investigated cell lines (A549, H292, H358, H460, H727, Calu-1, 16HBE, 1 HAEO, BEAS-2B and L-132) H292 and BEAS-2B cells displayed the relatively highest similarity in the expression of these genes with primary lung cell cultures and with human (nontumoral) lung tissue (Table 8).

\section{Primary cells in culture}

Cell lines are easier to handle than primary cells and primary cells can have large donor-to-donor variability (Table 9), which, depending on the problem in question, may be an advantage or disadvantage and, if the latter is the case, may be overcome by pooling. However, primary cells have the distinct advantage of a better conservation of in vivo parameters.

Important for studies on xenobiotic metabolism, unbroken cells (beside primary cells discussed in this chapter also including lung slices and other systems discussed further below) largely maintain the physiological concentrations 
of cofactors required for the activity of many xenobiotic metabolizing enzymes. The distortion of these concentrations leads to important distortions of toxicological consequences in many broken cell/acellular systems ]such as the Ames test performed under standard conditions (Glatt et al. 1981)].

Nevertheless, primary cells in culture can also quite dramatically change their properties. Thus, it has been reported by Elbert et al. (1999) that human alveolar type II cellsisolated from normal human distal lung tissue by enzyme treatment and subsequent purification - developed in culture to monolayers, which consisted predominantly of type I cells with only some interspersed type II cells. The authors state that this mimics well the situation in vivo [type II cells are the progenitors of type I cells and relatively easily acquire type I properties during culture (Demling et al. 2006; Swain et al. 2008)]. On the other hand, it is advisable to keep in mind that alveolar type II cells are relatively rich in xenobiotic metabolizing enzymes while alveolar type I cells are practically devoid of xenobiotic metabolizing enzymes [an aspect which was outside the scope of the absorption and transport study and was not investigated by the authors (Elbert et al. 1999)].

Thus, a serious limitation consists in (potential) loss of xenobiotic metabolizing enzyme activities during the culture time. Thus, Dimova et al. (2001) report that in primary type II pneumocytes in culture PROD and BROD activities decreased at 24 h by $84 \%$ and $82 \%$, respectively and continued to decline with no measurable PROD activity left at $48 \mathrm{~h}$. GST activity declined by 25 and $42 \%$ at 24 and $48 \mathrm{~h}$, respectively. NQO activity increased at $24 \mathrm{~h}$ by $55 \%$ (EROD activities were not detected).

In addition it is important to realize that primary cells in culture may upon exposure to xenobiotics under investigation be transformed to malignant cells/cell lines with correspondingly profound changes in their phenotypic properties/enzymes expressed (Hasegawa et al. 1988) as specifically observed for airway epithelial cells in culture (Ensell et al. 1998; Damiani et al. 2008). Stably transformed human bronchial epithelial cell lines have actually been generated this way (Bersaas et al. 2016). Gao et al. (2018) reported that hypermethylation of the $p g c p$ gene (plasma glutamate carboxypeptidase) is specifically associated with the immortalisation of human bronchial epithelial cells.

Newland et al. (2011) observed in NHTBE (primary human tracheobronchial epithelial cells) [obtained from Lonza (Lonza Group Ltd., Switzerland)], which they cultured in presence of retinoic acid at the air-liquid interphase, stable expression over their entire culture period of 28 days of mRNA coding for CYP1A1/1B1 and CYP2A6/2A13, CYps which are relevant for several lung toxins. In addition they obtained clearly measurable catalytic activity also over the entire culture period of 28 days for the CYP2A6/2A13 prototypic substrate coumarin and after treatment with the inducer TCDD also for Luc-CEE, a CY1A1/1B1 luminogenic probe. This was clearly superior in comparison with the frequently used human lung cell lines A594 and NCIH292 where these activities were considerably lower or absent.

Runge et al. (2001) found in primary cultures of NHBE CYP2B7, 2E1 and 2F1 transcripts. They observed CYP2Eprototypical chlorzoxazone 6-hydroxylation in these primary cultures where ethanol treatment led within 4 days to an up to 5 -fold increase of this activity.

Weems and Yost (2010) showed in NHBE that CYP1A1 and CYP2F1 mRNA expression was increased by metabolites of the pneumotoxin 3-methylindole, the former dependent on AhR activation, but the latter not.

Han et al. (2005) observed in NHBE (normal human bronchial epithelial cells) (primary cultures obtained from the commercial source BioWhittaker, Inc., Walkersville, Maryland, USA) an interesting cross talk between classical CYP1 inducers (cigarette smoke in this case) and estrogen. They observed binding of transfected estrogen receptor $\alpha$ to the CYP1B1 promoter near the transcription start site. The transfection with the estrogen receptor $\alpha$ led to a 4-fold increase in the production of CYP1B1 transcripts (CYP1B1 protein 6.5 -fold). The cigarette smoke extract-induced expression of CYP1B1 mRNA was further increased by estrogen receptor $\alpha$ (2.3-fold). Estrogen receptor $\alpha$ did not change the production of CYP1A1 transcripts, but that of its protein (2-fold on cigarette smoke extract exposure, 6.2-fold on estrogen exposure). The authors conclude that estrogen receptor $\alpha$ contributes to the regulation of CYP1B1 expression at the transcriptional level and to that of CYP1A1 on the translational level. They speculate that this contribution to the CYP1 expression control may contribute to the known higher DNA adduct levels in smoking females compared with men (Mollerup et al. 2006) and eventually also the greater susceptibility of (non-smoking) females to lung cancer (Freedman et al. 2008).

Nakayama Wong et al. (2011) reported an increase in the CYP1A1 and CYP1B1 mRNA level in human bronchial epithelial cells upon exposure to wildfire fine particulate matter. This increase was attenuated by the deferoxamine mesylatemediated Fenton reaction inhibition and the thereby inhibited ROS production, but interestingly not by the cell membrane-permeable oxygen radical scavenger $N$-acetyl-Lcysteine. The authors speculate that the known influence of $N$-acetyl-L-cysteine on various signal transduction pathways may have overwhelmed its radical-scavenging-based potential effect on the CYPIAI and CYPIB1 gene expression.

For the study by Courcot et al. (2012), which observed that four primary cell cultures of human bronchial epithelial cells were much superior to ten commonly utilized lung cell lines in mimicking human lung tissue with respect to 
expression of 380 genes coding for xenobiotica metabolizing enzymes (and transporters), see preceding chapter (Table 8).

Primary lung cells may be obtained by fiberoptic bronchoscopy from volunteers or may be commercially obtained from, e.g. Epithelix, BioWhittaker, Lonza or MatTek.

\section{Lung explant cultures and lung slices}

Lung explants and lung slices provide the advantage that these models largely maintain the original organ architecture (albeit not at the cut surfaces, but these may be kept small as related to the entire mass of the tissue).

On the other hand, these models do not maintain the physiological inflow and outflow of liquid or the physiological exposure of the lung to air versus blood in the alveoli. In addition, the relatively short-term viability of these models may, depending on the problem in question, represent a further limitation.

\section{Explants}

Autrup et al. (1980) used already in 1980 cultured human tracheobronchial explants to compare BP metabolism in human, mouse, rat, hamster and bovine explants. Most strikingly, they found that the total BP metabolism was high in human and in those animal species, which were known to be susceptible to BP-induced lung cancer formation. In all investigated species a high proportion of $\mathrm{BP}$ was metabolized to glutathione conjugates and to sulfates, much more than to glucuronides.

In human lung explant cultures, CYP1A1 was consistently induced by benzo[a]pyrene and also by TCDD (2,3,7,8-tetrachlorodibenzo-p-dioxin) and TCDD-like agonists (Wei et al. 2002).

\section{Precision-cut lung slices}

Precision-cut tissue slices are used for a wide range of pharmacological and toxicological investigations (Bach et al. 1996; Sturton et al. 2008), most of all as part of an in vitro test battery in an attempt to reduce the number of the animals required for insuring safety or to eventually completely replace them (Hess et al. 2016). The standard conditions for precision-cut lung slices use a thickness $(500 \mu \mathrm{m})$ and diameter $(6-10 \mathrm{~mm})$ to allow on the one hand diffusion of the substrates to the innermost cells and on the other hand allow for the maintenance of the organ architecture and a tolerably small contribution of damaged cells at the cutting edges (de Kanter et al. 2002a). Moreover, the presence of the natural matrix and other cell types is advantageous, since it can strongly influence xenobiotic metabolism (Slaus et al. 2001 and references therein).
De Kanter et al. (1999) demonstrated the usefulness of lung slices for the study of xenobiotic metabolism by showing in human (and rat) lung (and liver) slices active phase I (hydroxylation, $O$ - and $\mathrm{N}$-dealkylation) and phase II (glucuronidation and sulfonylation) metabolism using as substrates 7-ethoxycoumarin, 7-hydroxycoumarin, testosterone and lidocaine. Ioannides (2013) reviewed the available information of his studies and from studies of others reported in the literature. He concluded that precision-cut slices of lung (and liver and intestine) of human origin (as well as slices of various experimental animal species) respond to known, standard xenobiotic metabolizing enzyme inducers adequately mimicking the in vivo situation.

Human pulmonary metabolism has been shown for quite a number of pharmaceutical drugs using precisioncut human lung slices (e.g. Nave et al. 2006; Pavek and Dvorak 2008; Yilmaz et al. 2019).

\section{3-dimensional cultures, cultures at the air-liquid interphase, co-cultures, lung-on-a-chip}

3-dimensional (3D) cultures are now increasingly used and novel improvements of the techniques (e.g. Koban et al. 2018) may increase their future usefulness. A 3D human airway model was reported to predict respiratory toxicity of inhaled compounds with high accuracy (Sivars et al. 2018).

In the following, some examples will be presented regarding the use of 3D cultures for investigations on xenobiotic metabolism in the human lung:

Carterson et al. (2005) developed a 3-D (3-dimensional) A549 system and reported that this system showed increased expression of epithelial cell-specific markers and less cancer-specific markers, which are expressed in this cell line in their standard maintenance as monolayers. Choe et al. (2006) established a 3-D culture system using tissue-engineered matrices to support airway cells. Fully differentiated and well-characterized 3-D NHT/BE (normal human tracheal/ bronchial epithelial) primary human cell culture systems are commercially available (EpiAirway ${ }^{\circledR}$ from MatTek and MucilAir $^{\mathrm{TM}}$ from Epithelix). The latter has been shown to retain a pseudostratified morphology and appropriate functional characteristics up to 6 months (see below).

With respect to xenobiotica-metabolizing enzymes Baxter et al. (2015) reported that the commercially available 3D reconstituted respiratory epithelia system MucilAir ${ }^{\mathrm{TM}}$ expressed CYP transcripts similar to normal human lung. Thus, CYP1A1 and 1B1 were expressed and induced by TCDD. Specific respiratory epithelium CYPs such as CYP2A13, 2F1 and 3A5 were clearly expressed. The cells possessed catalytic CYP1A1/1B1 activities (shown using Luc-CEE as luminogenic probe substrate), as well as CYP2A6/2A13 catalytic activities (shown using coumarin 
as probe substrate). The cells maintained these activities for at least 1 month.

Formation of spheroids appears to offer some advantage. Takahashi et al. (2004) reported that SP-C mRNA, an alveolar type II cell-specific protein, is only expressed in culture when alveolar type II cells form a spheroid. Spheroids can be obtained from bronchial brushings. They develop cilia on the outside and are free floating (Deslee et al. 2007). When grown on matrigel, primary human bronchial epithelial cells can form spheroids, which secrete zink-a2-glycoprotein, lactoferrin and lysozyme (markers of glandular serous cells) and MUC5B mucin (marker of glandular mucous cells) (Wu et al. 2011).

A further step attempts to generate lung-derived in vitro systems with exposure to oxygen reminiscent of the in vivo situation by positioning the cells at the air-liquid interface. The apical side of the epithelium is exposed to air (5\% $\mathrm{CO}_{2}$ in humidified air) while the basolateral portions are immersed in the culture medium. Such cultures show well-differentiated epithelial morphology (Adler et al. 1990; Ehrhardt et al. 2002; Mathia et al. 2002). Inclusion of several constituents, most importantly retinoic acid, allows for reproducible differentiation. The normally used procedure consists in first growing the cells submerged until they reach confluency within 5-7 days, then raising the cells to the liquid-air interphase where they reach full differentiation within normally 2-3 weeks. Bronchial epithelial cells differentiate into a pseudo-stratified layer with ciliated columnar cells and mucus-secreting goblet cells providing the mucociliary function (Pezzulo et al. 2010). Cells grown at the liquid-air interphase possess an apical surface closer to that seen in vivo. This is reported to lead to a better protection against harmful insult from xenobiotic compounds reminiscent of the in vivo situation (BéruBé et al. 2009). Toxic effects of compounds applied as vapors are increasingly tested in human primary bronchial epithelial cells cultured at the air-liquid interface (e.g. Dwivedi et al. 2018).

With respect to xenobiotica-metabolizing enzymes Boei et al. (2017) compared human bronchial epithelial cells at different time points kept in 3D cultures and showed the following: during differentiation of submerged primary cells and thereafter as air-liquid interface cultures CYP2B6, 2F1, 4B1, 4X1 and 4Z1 transcripts were increased following the mucociliary differentiation of airway epithelial cells into a pseudo-stratified epithelial layer. Simultaneously, levels of ADH1C, ALDH1A1 and GSTA1 transcripts were increased as well. Differences between cultures originating from different donors were small. At early stages of differentiation catalytic activities of the investigated xenobiotica-metabolizing CYPs were absent. However, the mature air-liquid interface bronchial epithelial cultures efficiently metabolized the prototypical substrates for CYP1A2, 2A6, 2B6, 2C9 and $3 \mathrm{~A} 4$ phenacetin, coumarin, bupropion, diclofenac and midazolam, respectively, but not the prototypical substrates for CYP2C19, 2D6 and 2E1 mephenytoin, bufuralol and chlorzoxazone, respectively.

Co-cultures (Hermanns et al. 2009, 2010; Kasper et al. 2011), even triple co-cultures (Rothen-Rutishauser et al. 2005, 2008; Gehr et al. 2006; Lehmann et al. 2011; Hoang et al. 2012; Farcal et al. 2013) and quadruple co-cultures (Alfaro-Moreno et al. 2008; Klein et al. 2013) have been developed. Klein et al. (2011) have reviewed the potential usefulness of various co-culture models to study inflammatory and sensitizing effects of particles on the lung and their underlying mechanisms.

Of late, a co-culture system of human alveolar epithelial (hAELVi) and macrophage (THP-1) cell lines was established (Kletting et al. 2018). A 3D tetraculture alveolar model cultivated at the air-liquid interface was recently evaluated for its usefulness to score diesel exhaust particulate matter toxicity and found to produce a realistic response (Fizeşan et al. 2018). A co-culture system of differentiated airway epithelium and a fibroblast-containing subepithelial matrix is commercially available from MatTek (EpiAirwayFT) and from Epithelix (MucilAir-HF).

An example concerning xenobiotic metabolism: Iskandar et al. (2015) compared the consequences of cigarette smoke exposure for a monoculture of human bronchial epithelial cells without fibroblasts (MucilAir purchased from Epithelix Sarl, Geneva, Switzerland) with a coculture which in addition contained human fibroblasts (MucilAir-HF from the same source) on several parameters including xenobiotic metabolism parameters (CYP1A/1B activity using the luminogenic CYP1A/1B substrate luciferin-6'-chloroethyl ether as well as transcriptomics). Influence on stress parameters was less pronounced in the co-culture, possibly due to a protection by the fibroblasts (making the co-culture system less sensitive). On the other hand, in the co-culture effects of cigarette smoke on the xenobiotic metabolism parameters investigated were closer to those seen in vivo than in monoculture.

In order to take xenobiotic metabolism into account a co-culture of NHBE (normal human bronchial epithelial) cells with human primary hepatocytes was developed, called Metabo-Lung (Cardiff university). Combining the cells with the highest biotransformation potential, hepatocytes, with bronchial epithelial cells to at least partially account for specific local metabolism in the lung, represents a reasonable step toward getting closer to what might be expected from the complex interactions in the human organism to ultimately be present in the lung as metabolites generated from xenobiotic compounds. Bérubé (2011) states that primary human bronchial cells co-cultured at the air-liquid interphase with primary human hepatocytes achieve xenobiotic metabolism comparable to the in vivo situation. On the other hand, this system is obviously not suitable to recognize 
specific pulmonary metabolism. The author states that in the absence of hepatocytes no xenobiotic metabolism was observed in this culture. However, some xenobiotic metabolism has clearly been observed in human bronchial epithelial cells in culture (see above).

Organ-on-a-chip models attempt to even more accurately mimick the in vivo situation in in vitro models (Van Duinen et al. 2015; Ahadian et al. 2018; Rothbauer et al. 2019). Lung-on-a-chip models were established with alveolar epithelial cells on the apical and endothelial cells on the basolateral side of a porous membrane combined with supply of nutrition by microfluidic flow of medium (Huh et al. 2010, 2012; Esch et al. 2015; Stucki et al. 2015). Even breathing was mimicked in a co-culture of human alveolar epithelial cells on one side of a flexible, porous and gas-permeable elastomer and pulmonary microvascular endothelial cells on the other side combined with a computer-controlled cyclic vacuum suction allowing for "breathing"-associated tissue deformation (Huh et al. 2010). In addition, human ESC (embryonic stem cells) (Wang et al. 2007) and human iPSC (induced pluripotent stem cells) (Ghaedi et al. 2013) have been differentiated to alveolar epithelial cells. All of these developments focus largely on lung-related therapy/lung tissue engineering or on problems of transport/tissue barrier and particle toxicity and as main site to be mimicked largely on alveoli. These sophisticated systems may also prove useful for studies on xenobiotica-metabolizing enzymes and their catalytic activities, although it must be remembered that (beside alveolar type 2 cells) the major site of xenobiotic metabolism in the human lung are Clara (Club) cells in the bronchioles. In this respect it is worthy of note that Van Haute et al. (2009) generated from human ESC (embryonic stem cells) lung epithelial-like tissue differentiating into the major cell types of pulmonary epithelium including Clara cells, expression of the Clara cell-specific marker CC16 peaking at day 10 to a 1000 -fold increase, then decreasing to about 10-fold at day 20 in air-liquid interphase culture.

In addition, some transgenic models have been generated, expressing human lung CYPs in the mouse. Thus, Wei et al. (2012) reported the successful generation of a CYP2A13/2B6/2F1-transgenic mouse model expressing the human lung CYPs with a tissue distribution agreeing well with the human respiratory-tract selective expression of CYP2A13 and CYP2F1 (and with the expression of CYP2B6 in the liver). Catalytic activity of the CYP2A13 substrate NNK was observed.

\section{Conclusions}

The lung is a very complex organ possessing several different substructures, each of which with a considerable number of different cell types which, in turn, populate various regions of the substructures in differing ratios and densities.

From what is detailed in this review it becomes obvious that these several substructures and within them the various cell types possess a different pattern of xenobiotica-metabolizing enzymes, but that we are far from a complete picture. Available information centres mostly around xenobioticametabolizing CYPs and largely ignores the vast realm of other xenobiotica-metabolizing enzymes which in many cases will be decisive for the problem to be solved.

Several compounds are selectively toxic to the lung because of the specific or selective presence in the lung (quite often even in susceptible substructures/cell types) of toxifying xenobiotica-metabolizing enzymes. In the rabbit 4-ipomeanol is toxified by the relatively large and constitutive amounts of CYP2B1 and CYP4B1 in the Clara cells (nonciliated epithelial cells situated in the bronchioles of the terminal airways), which explains the selective toxicity of 4-ipomeanol to this region of the rabbit lung (Smith and Brian 1991). Several xenobiotic compounds are toxic to the lung because of the lung-selective presence of CYP2F subfamily members (Lanza et al. 1999; Wang et al. 1998). Many $\mathrm{PAH}$ are converted to the ultimate mutagens/carcinogens by members of the CYP1 family, which are highly inducible by these PAH in the lung (Shimada et al. 1996b).

Obviously, biomedical experiments and analysis directly in living human beings are limited by ethical and practical constraints. In vitro models using materials of human origin are lacking the complex interactions of the complete human organism. Whole-body non-human animal species suffer from extensive species differences.

As far as the topic of this review is concerned, one key question becomes which of presently available replacement systems comes closest to the situation in the living human organism with respect to xenobiotica-metabolizing enzymes in the lung. Due to the above-discussed complexity of the organ, lung, this will be different for any different problem to be solved. On grounds of the paucity of comparable information available on the enormous realm of xenobiotica-metabolizing enzymes in the various substructures and cell types of the living human lung it is obviously difficult to recommend individual systems to substitute for the living human lung. Table 11 attempts to approximate an estimation of relative suitability of experimental animal species and in vitro models with respect to various xenobiotic metabolizing enzymes in the human lung. The comparisons of the various systems with the human lung are obviously only valid if the lung as a whole organ is in question, such as in considerations of the 
Table 11 Attempt to approximate an estimation of relative suitability of experimental animal species or in vitro models with respect to various xenobiotic metabolizing enzymes in the human lung

\begin{tabular}{|c|c|}
\hline Enzyme & Presumably suitable models ${ }^{\mathrm{a}}$ \\
\hline CYP $^{\mathrm{b}}$ broad spectrum ${ }^{\mathrm{c}}$ & Monkey, (A549 cell line) ${ }^{\mathrm{a}}$ \\
\hline CYP 1 family ${ }^{\mathrm{d}}$ & Monkey, mouse, rat, rabbit \\
\hline $\mathrm{CYP} 1 \mathrm{~A} 2^{\mathrm{e}}$ & Rat \\
\hline CYP1A/3A ${ }^{\mathrm{f}}$ & A549 cell line \\
\hline CYP2E1 ${ }^{\mathrm{g}}$ & $\underline{\text { Rat }}$ \\
\hline EPHX1 $(\mathrm{mEH})^{\mathrm{h}}$ & $\underline{\text { Mouse, }}$ rat \\
\hline Cytosolic GST ${ }^{\mathrm{i}}$ & $\begin{array}{l}\text { Alveolar macrophages, } \\
\text { rat, } \mathbf{\text { mouse }} \text { (primary alveolar type II } \\
\text { cells) }\end{array}$ \\
\hline $\mathrm{UGT}^{\mathrm{k}}$ & A549 cell line, (mouse) \\
\hline
\end{tabular}

According to present state of information. To be taken with great caution, because of very limited comparability of data in most cases (see data and footnotes in Tables 3, 4, 5, 6, 7, 10 and text). Selected for this table if activities in model compared with human lung appear to be within one order of magnitude, underlined if within a factor of 3 , underlined and bold letters if within a factor of 2 (in brackets if just marginally over a factor of 10). Only applicable if the lung as entire organ is in question, i.e. not applicable for a defined compartment/ subcompartment such as bronchiolar Clara cells

a 4- $M U$ 4-methylumbelliferone, $A H H$ aryl hydrocarbon hydroxylase, $C D N B$ 1-chloro-2,4-dinitrobenzene, $C Y P$ cytochrome P450, ECOD 7-ethoxycoumarin $O$-deethylase, $E R O D$ 7-ethoxyresorufin $O$-deethylase, EPHX1 mEH, microsomal epoxide hydrolase, GST glutathione $S$-transferase, $M R O D$ 7-methoxyresorufin $O$-demethylase, $U G T$ UDPglucuronosyltransferase

${ }^{\mathrm{b}}$ Estimated using ECOD activities (preferentially catalyzed by CYP1, CYP2B, Cyp2D6, CYP3A)

${ }^{c}$ Estimated using AHH and EROD activities. Not applicable for CYP1A2 (based on MROD activities). Reliability of numbers very limited because of technical difficulties of accurate determination of very low CYP activities

${ }^{\mathrm{d}}$ Estimated using MROD activities

${ }^{\text {e}}$ Estimated using testosterone $6 \beta$-hydroxylation activity

${ }^{\mathrm{f}}$ Estimated using butadiene epoxide (mono-epoxide) as substrate

${ }^{\mathrm{g}}$ Estimated using benzo[a]pyrene as substrate

${ }^{\mathrm{h}}$ Estimated using CDNB as substrate

${ }^{i}$ Estimated using 1-naphthol (mouse) or 4-methylumbelliferone (A549 cells) as substrate

contribution (or first pass effect) of the lung to the metabolism of, e.g. an inhaled drug, but not if the metabolism in a compartment or subcompartment of the lung is crucial, such as in the local metabolism-dependent local toxicity of a xenobiotic compound to a defined region, such as the Clara cells (CLUB cells) of the bronchioli. With this limitation in mind, it is hoped that the data summarized in Table 11 may be helpful for selecting a model most likely to be suitable for a problem which crucially involves one or more of the enzymes evaluated in Table 11. However, it also becomes conspicuously clear from the overview in Table 11 that even not for all major xenobiotica-metabolizing enzymes sufficient comparable data are available for a meaningful comparison. Major missing enzymes include CYP2A, CYP2B, CYP2C, CYP2F, CYP4B, non-CYP mediated oxidoreductases, esterases, sulfotransferases and $N$-acetyltransferases (as far as some data are available they are discussed in the corresponding portions of the text, but these data are not straightforward comparable, e.g. not computable to rates of metabolite formation per weight of considered material). Much work is left to be performed.

An example of meritorious attempts in the right direction and of the remaining limitations are the studies on the pulmonary metabolism of styrene in the lung of the species susceptible to styrene-induced lung cancer, the mouse, contrasted with the metabolism in the resistant species, the rat, both of these compared with the human lung (discussed in the body of this review in the respective chapters, most comprehensively in the chapter concerning the mouse). It becomes obvious from this example that it is difficult to even know which metabolite(s) is/are key responsible for the problem in question (here, the resulting cancer). If we were in the position to know this, then the even more difficult question would become which ones of the great number of xenobiotica-metabolizing enzymes contribute substantially to the control of the responsible metabolite(s). Then a rational search could start which test system would come closest to the human living organism with regard to the problem in question. For this search, the information collected in this review will hopefully be useful as far as xenobioticametabolizing enzymes in the lung are concerned. Of course, in a real-world scenario entirely sufficient information may never be available. However, it is hoped that the information collected in this review will help to at least choose a system, which according to present-day information meets relatively best the requirements of an appropriate tool for solving a concrete problem.

\section{Compliance with ethical standards}

Conflict of interest RL and EF are employees of BASF SE, a chemical company that produces compounds to which human exposure may be by inhalation.

Open Access This article is distributed under the terms of the Creative Commons Attribution 4.0 International License (http://creativeco mmons.org/licenses/by/4.0/), which permits unrestricted use, distribution, and reproduction in any medium, provided you give appropriate credit to the original author(s) and the source, provide a link to the Creative Commons license, and indicate if changes were made. 


\section{References}

Abdull Razis AF, Bagatta M, De Nicola GR, Iori R, Ioannides C (2011) Up-regulation of cytochrome P450 and phase II enzyme systems in rat precision-cut rat lung slices by the intact glucosinolates, glucoraphanin and glucoerucin. Lung Cancer 71:298-305. https ://doi.org/10.1016/j.lungcan.2010.06.015

Adams DJ, Oesch F, Hartmann R, Wolf CR (1985) Induction and suppression of cytochromes P-450 in rat tissues. Biochem Soc Transact 13:358

Adissu HA, Schuller HM (2004) Antagonistic growth regulation of cell lines derived from human lung adenocarcinomas of Clara cell and alveolar type II cell lineage: implications for chemoprevention. Int J Oncol 24:1467-1472

Adler KB, Cheng PW, Kim KC (1990) Characterization of guinea pig tracheal epithelial cells maintained in biphasic organotypic culture:cellular composition and biochemical analysis of released clycoconjugates. Am J Respir Cell Mol Biol 2:145-154

Ahadian S, Civitarese R, Bannerman D, Mohammadi MH, Lu R, Wang E, Davenport-Huyer L, Lai B, Zhang B, Zhao Y, Mandla S, Korolj A, Radisic M (2018) Organ-on-a-chip platforms: a convergence of advanced materials, cells, and microscale technologies. Adv Healthc Mater. https://doi.org/10.1002/adhm.201800734

Alfaro-Moreno E, Nawrot TS, Vanaudenaerde BM et al (2008) Cocultures of multiple cell types mimic pulmonary cell communication in response to urban PM10. Eur Respir J 32:1184-1194. https://doi.org/10.1183/09031936.00044008

Anderson LM, Ward JM, Park SS, Jones AB, Junker JL, Gelboin HV, Rice JM (1987) Immunohistochemical determination of inducibility phenotype with a monoclonal antibody to a methyl-cholanthrene-inducible isozyme of cytochrome P-450. Cancer Res 47:6079-6085

Anttila S, Vainio H, Hietanen E, Camus AM, Malaveille C, Brun G, Husgafvel-Pursiainen K, Heikkila L, Karjalainen A, Bartsch H (1992) Immunohistochemical detection of pulmonary cytochrome P450IA and metabolic activities associated with P4501A1 and P4501A2 isozymes in lung cancer patients. Environ Health Perspect 98:179-182

Anttila S, Hirvonen A, Vainio H, Husgafvel-Pursiainen K, Hayes JD, Ketterer B (1993) Immunohistochemical localization of glutathione $S$-transferases in human lung. Cancer Res 53:5643-5648

Anttila S, Hukkanen J, Hakkola J, Stjernvall T, Beaune P, Edwars RJ, Boobis AR, Pelkonen O, Raunio H (1997) Expression and localization of CYP3A4 and CYP3A5 in human lung. Am J Respir Cell Mol Biol 16:242-249

Anttila S, Lei XD, Elovaara E, Karjalainen A, Sun W, Vainio H, Hankinson O (2000) An uncommon phenotype of poor inducibility of CYP1A1 in human lung is not ascribable to polymorphisms in the AHR, ARNT, or CYP1A1 genes. Pharmacogenetics 10:741-751

Anttila S, Tuominen P, Hirvonen A, Nurminen M, Karjalainen A, Hankinson O, Elovaara E (2001) CYP1A1 levels in lung tissue of tobacco smokers and polymorphisms of CYP1A1 and aromatic hydrocarbon receptor. Pharmacogenetics 11:501-509

Anttila S, Hakkola J, Tuominen P, Elovaara E, Husgafvel-Pursiainen K, Karjalainen A, Hirvonen A, Nurminen T (2003) Methylation of cytochrome $\mathrm{P} 4501 \mathrm{~A} 1$ promoter in the lung is associated with tobacco smoking. Cancer Res 63:8623-8628

Asikainen A, Tarhanen J, Poso A, Pasanen M, Alhava E, Juvonen RO (2003) Predictive value of comparative molecular field analysis modelling of naphthalene inhibition of human CYP2A6 and mouse CYP2A5 enzymes. Toxicol In Vitro 17:449-455

Autrup H, Wefald FC, Jeffrey AM, Tate H, Schwartz RD, Trump BF, Harris CC (1980) Metabolism of benzo[a]pyrene by cultured tracheobronchial tissues from mice, rats, hamsters, bovines and humans. Int J Cancer 25:293-300

Awasthi YC, Sing SV, Moller PC (1984) 1mmunochemical localization of glutathione $S$-transferases in rat lung. Lung 162:305-313

Bach PH, Vickers AEM, Fisher R, Baumann A, Brittebo E, Carlile DJ, Koster HJ, Lake BJ, Salmon F, Sawyer TW, Skibinski G (1996) The use of tissue slices for pharmacotoxico logical studies. The report and recommendations of ECVAM Workshop 20. ATLA 24:893-923

Baldwin RM, Jewell WT, Fanucchi MV, Plopper CG, Buckpitt AR (2004) Comparison of pulmonary/nasal CYP2F expression levels in rodents and rhesus macaque. J Pharmacol Exp Ther 309:127-136

Baldwin RM, Shultz MA, Buckpitt AR (2005) Bioactivation of the pulmonary toxicants naphthalene and 1-nitronaphthalene by rat CYP2F4. J Pharmacol Exp Ther 312:857-865

Barker DF, Walraven JM, Ristagno EH, Doll MA, States JC, Hein DW (2008) Quantitative tissue and gene-specific differences and developmental changes in Nat1, Nat2, and Nat3 mRNA expression in the rat. Drug Metab Dispos 36:2445-2451. https://doi. org/10.1124/dmd.108.023564

Baron J, Voigt JM (1990) Localization, distribution, and induction of xenobiotic-metabolizing enzymes and aryl hydrocarbon hydroxylase activity within lung. Pharmacol Ther 47:419-445

Bartsch H, Castegnaro M, Rojas M, Camus AM, Alexandrov K, Lang M (1992) Expression of pulmonary cytochrome P4501A1 and carcinogen DNA adduct formation in high risk subjects for tobacco-related lung cancer. Toxicol Lett. Spec No: 477-83

Bauer AK, Velmurugan K, Plöttner S, Siegrist KJ, Romo D, Welge P, Brüning T, Xiong KN, Käfferlein HU (2018) Environmentally prevalent polycyclic aromatic hydrocarbons can elicit cocarcinogenic properties in an in vitro murine lung epithelial cell model. Arch Toxicol 92:1311-1322. https://doi.org/10.1007/ s00204-017-2124-5

Baxter A, Thain S, Banerjee A, Haswell L, Parmar A, Phillips G, Minet E (2015) Targeted omics analyses, and metabolic enzyme activity assays demonstrate maintenance of key mucociliary characteristics in long term cultures of reconstituted human airway epithelia. Toxicol In Vitro 29:864-875. https://doi.org/10.1016/j. tiv.2015.03.004

Becker GM, Breeze RG, Carlson JR (1984) Autoradiographic evidence of 3-methylindole covalent binding to pulmonary epithelial cells in the goat. Toxicology 31:109-121

Beckett GJ, Howie AF, Hume R, Matharoo B, Hiley C, Jones P, Strange RC (1990) Human glutathione $S$-transferase: radioimmunoassay studies on the expression of Alpha-, Mu-, and Pi-class isoenzymes in developing lung and kidney. Biochim Biophys Acta 1036:176-182

Belinsky SA, White CM, Devereux TR, Swenberg JA, Anderson MW (1987) Cell selective alkyl- ation of DNA in rat Jung following low dose exposure to the tobacco specific carcinogen 4-( $N$-methyl- $N$-nitrosamino)-1-(3-pyridyl)-1-butanone. Cancer Res 47:1143-1148

Bentley P, Oesch F, Glatt HR (1977) Dual role of epoxide hydratase in both activation and inactivation. Arch Toxicol 39:65-75

Bernauer U, Heinrich-Hirsch B, Tönnies M et al (2005) Cytochrome P450 (CYP2E1)-dependent chlorzoxazone hydroxylase activities in human lung microsomes. Naunyn Schmiedebergs Arch Pharmacol 371:R93-R94

Bernauer U, Heinrich-Hirsch B, Tonnies M, Wolski PM, GundertRemy U (2006) Characterization of the xenobiotic-metabolizing cytochrome $\mathrm{P} 450$ expression pattern in human lung tissue by immunochemical and activity determination. Toxicol Lett $164: 278-288$ 
Bernauer U, Appel KE, Tönnies M (2009a) Expression and variability of microsomal epoxide hydrolase in human lung tissue. Toxicol Lett 189:S110-S111

Bernauer U, Settels E, Tönnies M, Appel KE (2009b) Detection of CYP2F1-associated enzyme activities in human lung microsomes. Naunyn-Schmiedebergs Arch Pharmacol 379:291-292

Bernstein J (1982) The role of the lung in the metabolism of ethanol. Res Commun Chem Pathol Pharmacol 38:43-56

Bersaas A, Arnoldussen YJ, Sjøberg M, Haugen A, Mollerup S (2016) Epithelial-mesenchymal transition and FOXA genes during tobacco smoke carcinogen induced transformation of human bronchial epithelial cells. Toxicol In Vitro 35:55-65. https://doi. org/10.1016/j.tiv.2016.04.012

BéruBé K (2011) Alternatives for lung research: stuck between a rat and a hard place. Altern Lab Anim 39:121-130

BéruBé K, Aufderheide M, Breheny D, Clothier R, Combes R, Duffin R, Forbes B, Gaça M, Gray A, Hall I, Kelly M, Lethem M, Liebsch M, Merolla L, Morin JP, Seagrave J, Swartz MA, Tetley TD, Umachandran M (2009) In vitro models of inhalation toxicity and disease. The report of a FRAME workshop. Altern Lab Anim 37:89-141

Bieche I, Narjoz C, Asselah T, Vacher S, Marcellin P, Lidereau R, Beaune P, de Waziers I (2007) Reverse transcriptase-PCR quantification of mRNA levels from cytochrome (CYP)1, CYP2 and CYP3 families in 22 different human tissues. Pharmacogenet Genom 17:731-742

Biggs JS, Wan J, Cutler NS, Hakkola J, Uusimaki P, Raunio H, Yost GS (2007) Mol Pharmacol 72:514-525

Bilimoria MH, Johnson J, Hogg JC, Witschi HP (1977) Pulmonary aryl hydrocarbon hydroxylase: tobacco smoke-exposed guinea pigs. Toxicol Appl Pharmacol 41:433-440

Billatos E, Faiz A, Gesthalter Y, LeClerc A, Alekseyev YO, Xiao X, Liu G, Ten Hacken NHT, Heijink IH, Timens W, Brandsma CA, Postma DS, van den Berge M, Spira A, Lenburg ME (2018) Impact of acute exposure to cigarette smoke on airway gene expression. Physiol Genom 50:705-713. https://doi.org/10.1152/ physiolgenomics.00092

Bock KW, von Clausbruch UC, Kaufmann R, Lilienblum W, Oesch F, Pfeil H, Platt KL (1980) Functional heterogeneity of UDP-glucuronyltransferase in rat tissues. Biochem Pharmacol 29:495-500

Boei JJWA, Vermeulen S, Klein B, Hiemstra PS, Verhoosel RM, Jennen DGJ, Lahoz A, Gmuender H, Vrieling H (2017) Xenobiotic metabolism in differentiated human bronchial epithelial cells. Arch Toxicol 91:2093-2105. https://doi.org/10.1007/s0020 4-016-1868-7

Bond JA (1983) Bioactivation and biotransformation of 1-nitropyrene in liver, lung and nasal tissue of rats. Mutat Res 124:315-324

Bond JA (1989) Review of the toxicology of styrene. Crit Rev Toxicol 19:227-249

Bond JA (1993) Metabolism and elimination of inhaled drugs and airborne chemicals from the lungs. Pharmacol Toxicol 72(Suppl 3):36-47

Bond JA, Mauderly JL (1984) Metabolism and macromolecular covalent binding of [14C]-1- nitropyrene in isolated perfused and ventilated rat lungs. Cancer Res 44:3924-3929

Bond JA, Medinsky MA (2001) Insights into the toxicokinetics and toxicodynamics of 1,3-butadiene. Chem Biol Interact 135-136:599-614

Bond JA, Mauderly JL, Henderson RF, McClellan RO (1985) Metabolism of 1-[14C]nitropyrene in respiratory tract tissue of rats exposed to diesel exhaust. Toxicol Appl Pharmacol 79:461-470

Bond JA, Harkema JR, Russell VI (1988) Regional distribution of xenobiotic metabolizing enzymes in respiratory airways of dogs. Drug Metab Dispos 16:116-124
Boogaard PJ, Bond JA (1996) The role of hydrolysis in the detoxification of 1,2:3,4-diepoxybutane by human, rat, and mouse liver and lung in vitro. Toxicol Appl Pharmacol 141:617-627

Boogaard PJ, Sumner SC, Bond JA (1996) Glutathione conjugation of 1,2:3,4-diepoxybutane in human liver and rat and mouse liver and lung in vitro. Toxicol Appl Pharmacol 136:307-316

Born SL, Caudill D, Fliter KL, Purdon MP (2002) Identification of the cytochromes $\mathrm{P} 450$ that catalyze coumarin 3,4-epoxidation and 3-hydroxylation. Drug Metab Dispos 30:483-487

Botto F, Seree E el Khyari S, Cau P, Henric A, De Meo M, Bergeron P, Barra Y (1994) Hypomethylation and hypoexpression of human CYP2E1 gene in lung tumors. Biochem Biophys Res Commun 205:1086-1092

Bourgine J, Billaut-Laden I, Happillon M, Lo-Guidice JM, Maunoury V, Imbenotte M, Broly F (2012) Gene expression profiling of systems involved in the metabolism and the disposition of xenobiotics: comparison between human intestinal biopsy samples and colon cell lines. Drug Metab Dispos 40:694-705

Boyd MR (1977) Evidence for the Clara cell as a site of cytochrome P450-dependent mixed-function oxidase activity in lung. Nature 269:713-715

Brown PJ, Bedard LL, Reid KR, Petsikas D, Massey TE (2007) Analysis of CYP2A contributions to metabolism of 4-(methylnitrosamino)-1-(3-pyridyl)-1-butanone in human peripheral lung microsomes. Drug Metab Dispos 35:2086-2094

Buckpit A, Chang AM, Weir A, Van Winkle L, Duan X, Philpot R, Plopper C (1995) Relationship of cytochrome P450 activity to Clara cell cytotoxicity. IV. Metabolism of naphthalene and naphthalene oxide in microdissected airways from mice, rats, and hamsters. Mol Pharmacol 47:74-81

Buckpitt A, Boland B, Isbell M, Morin D, Shultz M, Baldwin R, Chan K, Karlsson A, Lin C, Taff A, West J, Fanucchi M, Van Winkle L, Plopper C (2002) Naphthalene-induced respiratory tract toxicity: metabolic mechanisms of toxicity. Drug Metab Rev 34:791-820

Bui PH, Hankinson O (2009) Functional characterization of human cytochrome P450 2S1 using a synthetic gene-expressed protein in Escherichia coli. Mol Pharmacol 76:1031-1043

Canistro D, Bonamassa B, Pozzetti L, Sapone A, Abdel-Rahman SZ, Biagi GL, Paolini M (2009) Alteration of xenobiotic metabolizing enzymes by resveratrol in liver and lung of CD1 mice. Food Chem Toxicol. 47:454-461. https://doi.org/10.1016/j. fct.2008.11.040

Canistro D, Barillari J, Melega S, Sapone A, Iori R, Speroni E, Paolini M (2012) Black cabbage seed extract affects rat Cyp-mediated biotransformation: organ and sex related differences. Food Chem Toxicol 50:2612-2621. https://doi.org/10.1016/j.fct.2012.05.030

Cappiello M, Giuliani L, Rane A, Pacifici GM (1991) Dopamine sulfotransferase is better developed than $p$-nitrophenol sulfotransferase in the human fetus. Dev Pharmacol Ther 16:83-88

Carlson GP (1997) Effects of inducers and inhibitors on the microsomal metabolism of styrene to styrene oxide in mice. J Toxicol Environ Health 51:477-488

Carlson GP (1998) Metabolism of styrene oxide to styrene glycol by mouse liver and lung. J Toxicol Environ Health A 53(1):19-27 (Erratum in: J Toxicol Environ Health (1999) 57:443)

Carlson GP (2002) Effect of the inhibition of the metabolism of 4 -vinylphenol on its hepatotoxicity and pneumotoxicity in rats and mice. Toxicology 179:129-136

Carlson GP (2004) Comparison of the susceptibility of wild-type and CYP2E1 knockout mice to the hepatotoxic and pneumotoxic effects of styrene and styrene oxide. Toxicol Lett 150:335-339

Carlson GP (2008) Critical appraisal of the expression of cytochrome P450 enzymes in human lung and evaluation of the possibility that such expression provides evidence of potential styrene 
tumorigenicity in humans. Toxicology 254:1-10. https://doi. org/10.1016/j.tox.2008.09.017

Carlson GP, Hynes DE, Mantick NA (1998) Effects of inhibitors of CYP1A and CYP2B on styrene metabolism in mouse liver and lung microsomes. Toxicol Lett 98:131-137

Carlson GP, Mantick NA, Powley MP (2000) Metabolism of styrene by human lung and liver. J Toxicol Environ Health 59:591-595

Carlson GP, Ullman M, Mantick NA, Snyder PW (2002) 4-Vinylphenol-induced pneumotoxicity and hepatotoxicity in mice. Toxicol Pathol 30:565-569

Carr BA, Wan J, Hines RN, Yost GS (2003a) Characterization of the human lung CYP2F1 gene and identification of a novel lung-specific binding motif. J Biol Chem 278:15473-15483

Carr BA, Ramakanth S, Dannan GA, Yost GS (2003b) Characterization of pulmonary CYP4B2, specific catalyst of methyl oxidation of 3-methylindole. Mol Pharmacol 63:1137-1147

Carterson AJ, Hönerzu Bentrup K, Ott CM et al (2005) A549 lung epithelial cells grown as three-dimensional aggregates: alternative tissue culture model for Pseudomonas aeruginosa pathogenesis. Infect Immun 73:1129-1140

Cassel TN, Gustafsson JA, Nord M (2000) CYP2B1 is regulated by $\mathrm{C} / \mathrm{EBP}$ alpha and C/EBP delta inlung epithelial cells. Mol Cell Biol Res Commun 3:42-47

Castell JV, Donato MT, Gómez-Lechón MJ (2005) Metabolism and bioactivation of toxicants in the lung. The in vitro cellular approach. Exp Toxicol Pathol 57(Suppl 1):189-204

Chae YH, Yun CH, Guengerich FP, Kadular FF, El-Bayoumy K (1993) Roles of human hepatic and pulmonary cytochrome P450 enzymes in the metabolism of the environmental carcinogen 6-nitrochrysene. Cancer Res 53:2028-2034

Chae YH, Thomas T, Guengerich FP, Fu PP, Bayoumy K (1999) Comparative metabolism of 1-, 2-, and 4-nitropyrene by human hepatic and pulmonary microsomes. Cancer Res 59:1473-1480

Chan JK, Vogel CF, Baek J, Kodani SD, Uppal RS, Bein KJ, Anderson DS, Van Winkle LS (2013) Combustion derived ultrafine particles induce cytochrome P-450 expression in specific lung compartments in the developing neonatal and adult rat. Am J Physiol Lung Cell Mol Physiol 304:L665-L677. https://doi. org/10.1152/ajplung.00370.2012

Chang H, Chang LW, Cheng YH, Tsai WT, Tsai MX, Lin P (2006) Preferential induction of CYP1A1 and CYP1B1 in CCSPpositive cells. Toxicol Sci 89:205-213

Chiang HC, Wang CK, Tsou TC (2012) Differential distribution of CYP2A6 and CYP2A13 in the human respiratory tract. Respiration 84:319-326

Chikara S, Mamidi S, Sreedasyam A, Chittem K, Pietrofesa R, Zuppa A, Moorthy G, Dyer N, Christofidou-Solomidou M, Reindl KM (2018) Flaxseed consumption inhibits chemically induced lung tumorigenesis and modulates expression of phase ii enzymes and inflammatory cytokines in $\mathrm{A} / \mathrm{J}$ mice. Cancer Prev Res (Phila) 11:27-37. https://doi.org/10.1158/1940-6207. CAPR-17-0119

Chirulli V, Marvasi L, Zaghini A, Fiorio R, Longo V, Gervasi PG (2007) Inducibility of AhR-regulated CYP genes by $\beta$-naphthoflavone in the liver, lung, kidney and heart of the pig. Toxicology 240:25-37

Cho TM, Rose RL, Hodgson E (2006) In vitro metabolism of naphthalene by human liver microsomal cytochrome P450 enzymes. Drug Metab Dispos 34:176-183

Choe MM., Tomei AA, Swartz MA (2006) Physiological 3D tissue model of the airway wall and mucosa. Nat Protoc 1:357-362. http://www.nature.com/nprot/journal/v1/n1/suppinfo/nprot .2006.54_S1.html

Choudhary D, Jansson I, Schenkman JB, Sarfarazi M, Stoilov I (2003) Comparative expression profiling of 40 mouse cytochrome $\mathrm{P} 450$ genes in embryonic and adult tissues. Arch Biochem Biophys 414:91-100

Choudhary D, Jansson I, Stoilov I, Sarfarazi M, Schenkman JB (2005) Expression patterns of mouse and human CYP orthologs (families 1-4) during development and in different adult tissues. Arch Biochem Biophys 436:50-61

Chung WG, Park CS, Roh HK, Lee WK, Cha YN (2000) Oxidation of ranitidine by isozymes of flavin-containing monooxygenase and cytochrome P450. Jpn J Pharmacol 84:213-220

Cohen JT, Carlson G, Charnley G, Coggon D, Delzell E, Graham JD, Greim H, Krewski D, Medinski M, Monson R, Paustenbach D, Petersen B, Rappoport S, Rhomberg I, Ryan PB, Thompson K (2002) A comprehensive evaluation of the potential health risks associated with occupational and environmental exposure to styrene. J Toxicol Environ Health 5:1-265

Cossar D, Bell J, Strange R, Jones M, Sandison A, Hume R (1990) The alpha and pi isoenzymes of glutathione $S$-transferase in human fetal lung: in utero ontogeny compared with differentiation in lung organ culture. Biochim Biophys Acta 1037:221-226

Courcot E, Leclerc J, Lafitte JJ, Mensier E, Jaillard S, Gosset P, Shirali P, Pottier N, Broly F, Lo-Guidice JM (2012) Xenobiotic metabolism and disposition in human lung cell models: comparison with in vivo expression profiles. Drug Metab Dispos 40:1953-1965. https://doi.org/10.1124/dmd.112.046896

Crawford EL, Weaver DA, DeMuth JP, Jackson CM, Khuder SA, Frampton MW, Utell MJ, Thilly WG, Willey JC (1998) Measurement of cytochrome P450 2A6 and 2E1 gene expression in primary human bronchial epithelial cells. Carcinogenesis 19:1867-1871

Cruzan G, Cushman JR, Andrews LS, Granville GC, Johnson KA, Hardy CJ, Coombes DW, Mullins PA, Brown WR (1998) Chronic toxicity/oncogenicity study of styrene in CD rate by inhalation exposure for 104 weeks. Toxicol Sci 46:266-281

Cruzan G, Cushman JR, Andrews LS, Granville GC, Johnson KA, Bevan C, Hardy CJ, Coombs DW, Mullins PA, Brown WR (2001) Chronic toxicity/oncogenicity study of styrene in CD-1 mice by inhalation exposure for 104 weeks. J Appl Toxicol 21:185-198

Cruzan G, Carlson GP, Johnson KA, Andrews LS, Banton MI, Bevan C, Cushman JR (2002) Styrene respiratory tract toxicity and mouse lung tumors are mediated by CYP2F-generated metabolites. Reg Toxicol Pharmacol 35:308-319

Cruzan G, Carlson GP, Turner M, Mellert W (2005) Ring-oxidized metabolites of styrene contribute to styrene-induced Clara-cell toxicity in mice. J Toxicol Environ Health A 68:229-237

Cruzan G, Bus J, Banton M, Gingell R, Carlson G (2009) Mouse specific lung tumors from CYP2F2- mediated cytotoxic metabolism: an endpoint/toxic response where data from multiple chemicals converge to support a mode of action. Regul Toxicol Pharmacol 55:205-218. https://doi.org/10.1016/j.yrtph.2009.07.002

Cruzan G, Bus J, Hotchkiss J, Harkema J, Banton M, Sarang S (2012) CYP2F2-generated metabolites, not styrene oxide, are a key event mediating the mode of action of styrene-induced mouse lung tumors. Regul Toxicol Pharmacol 62:214-220. https://doi. org/10.1016/j.yrtph.2011.10.007

Csanady GA, Kessler W, Hoffmann HD, Filser JG (2003) A toxicokinetic model for styrene and its metabolite styrene-7,8-oxide in mouse, rat and human with special emphasis on the lung. Toxicol Lett 138:75-102

Csanády GA, Guengerich FP, Bond JA (1992) Comparison of the biotransformation of 1,3-butadiene and its metabolite, butadiene monoepoxide, by hepatic and pulmonary tissues from humans, rats and mice. Carcinogenesis 13:1143-1153

Csanády GA, Mendrala AL, Nolan RJ, Filser JG (1994) A physiologic pharmacokinetic model for styrene and styrene-7,8-oxide in mouse, rat and man. Arch Toxicol 68:143-157 
Czerwinski M, McLemore TL, Philpot RM, Nhamburo PT, Korzekwa K, Gelboin HV et al (1991) Metabolic activation of 4-ipomeanol by complementary DNA-expressed human cytochromes P-450: evidence for species-specific metabolism. Cancer Res 51:4636-4638

Czerwinski M, McLemore TL, Gelboin HV, Gonzalez FJ (1994) Quantification of CYP2B7, CYP2B1, and CYPOR messenger RNAs in normal human lung and lung tumors. Cancer Res 54:1085-1091

Dahl GA, Miller JA, Miller EC (1978) Vinyl carbamate as a promutagen and more carcinogenic analog of ethyl carbamate. Cancer Res 38:3793-3804

Dairou J, Petit E, Ragunathan N, Baeza-Squiban A, Marano F, Dupret JM, Rodrigues-Lima F (2009) Arylamine $N$-acetyltransferase activity in bronchial epithelial cells and its inhibition by cellular oxidants. Toxicol Appl Pharmacol 236(3):366-371. https://doi. org/10.1016/j.taap.2009.02.010

Damiani LA, Yingling CM, Leng S, Romo PE, Nakamura J, Belinsky SA (2008) Carcinogen-induced gene promoter hypermethylation is mediated by DNMT1 and causal for transformation of immortalized bronchial epithelial cells. Cancer Res 68:9005-9014

De Kanter R, Olinga P, De Jager MH, Merema MT, Meijer DK, Groothius GM (1999) Precision-cut organ slices as a tool to study toxicity and metabolism of xenobiotics with special reference to non-hepatic tissues. Toxicol In Vitro 13:737-744

De Kanter R, Monshouwer M, Meijer DK, Groothuis GM (2002a) Precision-cut organ slices as a tool to study toxicity and metabolism of xenobiotics with special reference to non-hepatic tissues. Curr Drug Metab 3:39-59

De Kanter R, De Jager MH, Draaisma AL, Jurva JU, Olinga P, Meijer DK, Groothuis GM (2002b) Drug-metabolizing activity of human and rat liver, lung, kidney and intestine slices. Xenobiotica 32:349-362

De Waziers I, Cugnenc PH, Yang CS, Leroux J-P, Beaune PH (1990) Cytochrome P 450 isoenzymes, epoxide hydrolase and glutathione transferases in rat and human hepatic and extrahepatic tissues. J Pharmacol Exp Ther 253:387-394

Dees JH, Coe LD, Yasukocki Y, Masters BS (1980) lmmunofluorescence of NADPH-cytochrome c (P-450) reductase in rat and minipig tissues injected with phcnobarbital. Science 208:1473-1475

Dees JH, Masters BS, Muller-Eberhard U, Johnson EF (1982) Effect of 2,3,7,8-tetrachlorodibenzo-p-dioxin and phenobarbital on the occurrence and distribution of four cytochrome P-450 isozymes in rabbit kidney, lung, and liver. Cancer Res 42(4):1423-1432

Delbressine LP, van Bladeren PJ, Smeets FL, Seutter-Berlage F (1981) Stereoselective oxidation of styrene to styrene oxide in rats as measured by mercapturic acid excretion. Xenobiotica 11:589-594

Demling N, Ehrhardt C, Kasper M et al (2006) Promotion of cell adherence and spreading: a novel function of RAGE, the highly selective differentiation marker of human alveolar epithelial type I cells. Cell Tissue Res 323:475-488. https://doi.org/10.1007/ s00441-005-0069-0

DePierre JW, Seidegård J, Morgenstern R, Balk L, Meijer J, Aström A, Norélius I, Ernster L (1984) Induction of cytosolic glutathione transferase and microsomal epoxide hydrolase activities in extrahepatic organs of the rat by phenobarbital, 3-methylcholanthrene and trans-stilbene oxide. Xenobiotica 14(4):295-301

Deslee G, Dury S, Perotin J et al (2007) Bronchial epithelial spheroids: An alternative culture model to investigate epithelium inflammation-mediated COPD. Respir Res 8:86. https://doi. org/10.1186/1465-9921-8-86

Devereux TR, Fouts JR (1981) Xenobiotic metabolism by alveolar type II cells isolated from rabbit lung. Biochem. Pharmacol 30:1231-1237
Devereux TR, Diliberto JJ, Fouts JR (1985) Cytochrome P-450 monooxygenase, epoxide hydrolase and flavin monooxygenase activities in Clara cells and alveolar type II cells isolated from the rabbit. Cell Biol. Toxicol 1:57-65

Devereux TR, Anderson MW, Belinsky SA (1988) Factors regulating activation and DNA alkylation by 4 -( $N$-methyl- $N$-nitrosamino)1-(3-pyridyl)-1-butanone and nitrosodimethylamine in rat lung and isolated Jung cells, and the relationship to carcinogenicity. Cancer Res 48:4215-4221

Devereux TR, Domin BA, Philpot RM (1989) Xenobiotic metabolism by isolated pulmonary cells. Pharmacol Ther 41(1-2):243-256

Dimova S, Hoet PH, Nemery B (2001) Xenobiotic-metabolizing enzyme activities in primary cultures of rat type II pneumocytes and alveolar macrophages. Drug Metab Dispos 29(10):1349-1354

Ding X, Kaminsky LS (2003) Human extrahepatic cytochrome P450: function in xenobiotic metabolism and tissue-selective chemical toxicity in the respiratory and gastrointestinal tracts. Annu Rev Pharmacol Toxicol 43:149-173

Dolphin CT, Beckett DJ, Janmohamed A, Cullingford TE, Smith RL, Shephard EA, Phillips IR (1998) The flavin-containing monooxygenase 2 gene (FMO2) of humans, but not of other primates, encodes a truncated, nonfunctional protein. J Biol Chem 273:30599-30607

Domanski TL, Finta C, Halpert JR, Zaphiropoulos PG (2001) cDNA cloning and initial characterization of CYP3A43, a novel human cytochrome P450. Mol Pharmacol 59(2):386-392

Domin BA, Devereux TR, Philpot RM (1986) The cytochrome P-450 monooxygenase system of rabbit lung enzyme components, activities, and induction in the nonciliated bronchiolar epithelial (Clara) cell, alveolar type II cell, and alveolar macrophage. Mol Pharmacol 30(3):296-303

Donato MT, Castell JV, Gomez-Lechon MJ (1999) Characterization of drug metabolizing activities in pig hepatocytes for use in bioartificial liver devices: comparison with other hepatic cellular models. J Hepatol 31:542-549

Dowsley TF, Reid K, Petsikas D, Ulreich JB, Fisher RL, Forkert P-G (1999) Cytochrome P-450-dependent bioactivation of 1,1-dichloroethylene to a reactive epoxide in human lung and liver microsomes. J Pharmacol Exp Ther 289:641-648

Dwivedi AM, Upadhyay S, Johanson G, Ernstgård L, Palmberg L (2018) Inflammatory effects of acrolein, crotonaldehyde and hexanal vapors on human primary bronchial epithelial cells cultured at air-liquid interface. Toxicol In Vitro 46:219-228

Ehrhardt C, Kneuer C, Fiegel J et al (2002) Influence of apical fluid volume on the development of functional intercellular junctions in the human epithelial cell line 16HBE14o-: implications for the use of this cell line as an in vitro model for bronchial drug absorption studies. Cell Tissue Res 308:391-400

Eke BC, Işcan M (2002) Effects of cigarette smoke with different tar contents on hepatic and pulmonary xenobiotic metabolizing enzymes in rats. Hum Exp Toxicol 21:17-23

Elbert KJ, Schäfer UF, Schäfers HJ, Kim KJ, Lee VH, Lehr CM (1999) Monolayers of human alveolar epithelial cells in primary culture for pulmonary absorption and transport studies. Pharm Res 16:601-608

El-Kabbani O, Dhagat U, Hara A (2011) Inhibitors of human $20 \alpha$-hydroxysteroid dehydrogenase (AKR1C1). J Steroid Biochem Mol Biol 125:105-111. https://doi.org/10.1016/j.jsbmb .2010 .10 .006

Endo S, Matsunaga T, Arai Y, Ikari A, Tajima K, El-Kabbani O, Yamano S, Hara A, Kitade Y (2014) Cloning and characterization of four rabbit aldo-keto reductases featuring broad substrate specificity for xenobiotic and endogenous carbonyl compounds: relationship with multiple forms of drug ketone 
reductases. Drug Metab Dispos 42(4):803-812. https://doi. org/10.1124/dmd.113.056044

Ensell MX, Whong WZ, Heng ZC, Nath J, Ong T (1998) In vitro and in vivo transformation in rat tracheal epithelial cells exposed to diesel emission particles and related compounds. Mutat Res 412:283-291

Esch EW, Bahinski A, Huh D (2015) Organs-on-chips at the frontiers of drug discovery. Nat Rev Drug Discov 14:248-260. https:// doi.org/10.1038/nrd4539

Falls JG, Cherrington NJ, Clements KM, Philpot RM, Levi PE, Rose RL, Hodgson E (1997) Molecular cloning, sequencing, and expression in Escherichia coli of mouse flavin-containing monooxygenase 3 (FMO3): comparison with the human isoform. Arch Biochem Biophys 347(1):9-18

Fanucchi MV, Buckpitt AR, Murphy ME, Plopper CG (1997) Naphthalene cytotoxicity in the differentiating Clara cells of neonatal mice. Toxicol Appl Pharmacol 144:96-104

Fanucchi MV, Buckpitt AR, Murphy ME, Storms DH, Hammock BD, Plopper CG (2000) Development of phase II xenobiotic metabolizing enzymes in differentiating murine clara cells. Toxicol Appl Pharmacol 168:253-267

Farcal LR, Uboldi C, Mehn D et al (2013) Mechanisms of toxicity induced by $\mathrm{SiO}_{2}$ nanoparticles of in vitro human alveolar barrier: effects on cytokine production, oxidative stress induction, surfactant proteins A mRNA expression and nanoparticles uptake. Nanotoxicology 7:1095-1110. https://doi. org/10.3109/17435390.2012.710658

Filser JG, Oberste-Frielinghaus HR, Dhawan-Robl M, Faller TH, Kessler W, Csanady GA (1999) A physiological toxicokinetic model for styrene and styrene-7,8-oxide in mouse, rat and human considering metabolism in the lung. GSF Institute of Toxicology, Neuherberg (quoted in Cohen et al. 2002)

Filser JG, Kessler W, Csanady GA (2002) Estimation of a possible tumorigenic risk of styrene from daily exposure via food and ambient air. Toxicol Lett 126:1-18

Fizeşan I, Chary A, Cambier S, Moschini E, Serchi T, Nelissen I, Kiss B, Pop A, Loghin F, Gutleb AC (2018) Responsiveness assessment of a 3D tetra-culture alveolar model exposed to diesel exhaust particulate matter. Toxicol In Vitro 53:67-79. https://doi.org/10.1016/j.tiv.2018.07.019

Fong AT, Rasmussen RE (1987) Formation and accumulation of 0 6-ethylguanine in DNA of enriched populations of Clara cells, alveolar type II cells, and macrophages of hamsters exposed to diethylnitrosamine. Toxicology 43:289-299

Forkert PG (1995) CYP2E1 is preferentially expressed in Clara cells of murine lung: localization by in situ hybridization and immunohistochemical methods. Am J Respir Cell Mol Biol 12:589-596

Forkert PG (1999) 1,1-Dichloroethylene-induced Clara cell damage is associated with in situ formation of the reactive epoxide. Immunohistochemical detection of its glutathione conjugate. Am J Respir Cell Mol Biol 20:1310-1318

Forkert P-G, Lee RP (1997) Metabolism of ethyl carbamate by pulmonary cytochrome $\mathrm{P} 450$ and carboxylesterase isozymes: involvement of CYP2E1 and hydrolase A. Toxicol Appl Pharmacol 146:245-254

Forkert PG, Vessey ML, Elce JS, Park SS, Gelboin HV, Cole SPC (1986) Localization of phenobarbital- and 3-methylcholanthrene-inducible cytochromes P-450 in mouse lung with monoclonal antibodies. Res Commun Chem Path Pharmac 53:147-157

Forkert PG, Vessey ML, Park SS, Gelboin HV, Cole SPC (1989) Cytochromes P-450 in murine lung: an immunohistochemical study with monoclonal antibodies. Drug Metab Dispos $17: 551-555$
Forkert PG, Premdas PD, Bowers RJ (2000) Epoxide formation from diallyl sulfone is associated with CYP2E1 inactivation in murine and human lungs. Am J Respir Cell Mol Biol 23:687-695

Forkert PG, Lee RP, Reid K (2001) Involvement of CYP2E1 and carboxylesterase enzymes in vinyl carbamate metabolism in human lung microsomes. Drug Metab Dispos 29:258-263

Foster JR, Elcombe CR, Boobis AR, Davies O, Sesardic O, Mcquade J, Robson RT, Hayward C, Lock EA (1986) lmmunocytochemical localization of cytochrome P-450 in hepatic and extra-hepatic tissues of the rat with a monoclonal antibody against cytochrome P-450 c. Biochem Pharmacol 35:4543-4554

Foster KA, Oster CG, Mayer MM, Avery ML, Audus KL (1998) Characterization of the A549 cell line as a type II pulmonary epithelial cell model for drug metabolism. Exp Cell Res 243:359-366

Freedman ND, Leitzmann MF, Hollenbeck AR, Schatzkin A, Abnet CC (2008) Cigarette smoking and subsequent risk of lung cancer in men and women: analysis of a prospective cohort study. Lancet Oncol 9:649-656

Fukami T, Katoh M, Yamazaki H, Yokoi T, Nakajima M (2008) Human cytochrome P450 2A13 efficiently metabolizes chemicals in air pollutants: naphthalene, styrene, and toluene. Chem Res Toxicol 21:720-725

Gadberry MG, DeNicola DB, Carlson GP (1996) Pneumo-toxicity and hepatotoxicity of styrene to styrene oxide. J Toxicol Environ Health 48:273-294

Gamage N, Barnett A, Hempel N, Duggleby RG, Windmill KF, Martin JL, McManus ME (2006) Human sulfotransferases and their role in chemical metabolism. Toxicol Sci 90:5-22

Gao C, Xing X, He Z, Chen S, Wang S, Li Q, Guo P, Zhang H, Li H, Chen L, Wang Q, Zhao J, Xiao Y, Chen W, Li D (2018) Gene 642:505-512

Gate L, Langlais C, Micillino JC, Nunge H, Bottin MC, Wrobel R, Binet S (2006) Bitumen fume-induced gene expression profile in rat lung. Toxicol Appl Pharmacol 215:83-92

Gehr P, Blank F, Rothen-Rutishauser B (2006) Fate of inhaled particles after interaction with the lung surface. Paediatr Respir Rev 7:S73-S75

Genter MB, Marlowe J, Kevin KJ et al (2006) Naphthalene toxicity in mice and aryl hydrocarbon receptor-mediated CYPs. Biochem Biophys Res Commun 348:120-123

Gerbal-Chaloin S, Daujat M, Pascussi JM, Pichard-Garcia L, Vilarem MJ, Maurel P (2002) J Biol Chem 277(1):209-217

Gervot L, Rochat B, Gautier JC, Bohnenstengel F, Kroemer H, de Berardinis V, Martin H, Beaune P, de Waziers I (1999) Human CYP2B6: expression, inducibility and catalytic activities. Pharmacogenetics 9:295-306

Ghaedi M, Calle EA, Mendez JJ, Gard AL, Balestrini J, Booth A, Bove PF, Gui L, White ES, Niklason LE (2013) Human iPS cellderived alveolar epithelium repopulates lung extracellular matrix. J Clin Invest 123:4950-4962. https://doi.org/10.1172/JCI68793

Gibbs-Flournoy EA, Gilmour MI, Higuchi M, Jetter J, George I, Copeland L, Harrison R, Moser VC, Dye JA (2018) Differential exposure and acute health impacts of inhaled solid-fuel emissions from rudimentary and advanced cookstoves in female CD-1 mice. Environ Res 161:35-48. https://doi.org/10.1016/j. envres.2017.10.043

Gill SS, Hammock BD (1980) Distribution and properties of a mammalian soluble epoxide hydrase. Biochem Pharmacol 29:389-395

Glatt HR, Billings R, Platt KL, Oesch F (1981) Improvement of the correlation of bacterial mutagenicity with carcinogenicity of benzo[a]pyrene and four of its major metabolites by activation with intact liver cells instead of cell homogenate. Cancer Res 41:270-277

Gram TE (1997) Chemically reactive intermediates and pulmonary xenobiotic toxicity. Pharmacol Rev 49:297-341 
Grasso P, Williams M, Hodgson R, Wright MG, Gangolli SD (1971) The histochemical distribution of aniline hydroxylase in rat tissues. Histochem J 3:117-126

Green T (2000) Pulmonary toxicity and carcinogenicity of trichloroethylene: species differences and modes of action. Environ Health Perspect 108(Suppl. 2):261-264

Green T, Mainwaring GW, Foster JR (1997) Trichloroethylene-induced mouse lung tumors: studies on the mode of action and comparisons between species. Fundam Appl Toxicol 37:125-130

Green T, Lee R, Toghill A, Meadowcroft S, Lund V, Foster J (2001a) The toxicity of styrene to the nasal epithelium of mice and rats: studies on the mode of action and relevance to humans. Chem Biol Interact 137:185-202

Green T, Toghill A, Foster JR (2001b) The role of cytochromes P-450 in styrene induced pulmonary toxicity and carcinogenicity. Toxicology 169:107-117

Greene JF, Zheng J, Grant DF, Hammock BD (2000) Cytotoxicity of 1,2-epoxynaphthalene is correlated with protein binding and in situ glutathione depletion in cytochrome P4501A1 expressing Sf-21 cells. Toxicol Sci 53:352-360

Guengerich FP (1977a) Preparation and properties of highly purified cytochrome P-450 and NADPH-cyto-chrome P-450 reductase from pulmonary microsomes of untreated rabbits. Mol Pharmacol 13:911-923

Guengerich FP (1977b) Separation and purification of multiple forms of microsomal cytochrome P-450: activities of different forms of cytochrome P-450 towards several compounds of environmental interest. J Biol Chem 252:3970-3979

Guengerich FP (1990) Purification and characterization of xenobiotic-metabolizing enzymes from lung tissue. Pharmacol Ther 45:299-308

Guengerich FP, Wang P, Davidson N (1982) Estimation of isozymes of microsomal cytochrome P-450 in rats, rabbits and humans using immunochemical staining coupled with sodium dodecyl sulfatepolyacrylamide gel electrophoresis. Biochemistry 21:1698-1706

Guengerich FP, Kim D-H, Iwasaki M (1991) Role of human cytochrome P-450IIE1 in the oxidation of many low molecular weight cancer suspects. Chem Res Toxicol 4:168-179

Guidice JM, Marez D, Sabbagh N, Legrand-Andreoletti M, Spire C, Alcaïde E, Lafitte JJ, Broly F (1997) Evidence for CYP2D6 expression in human lung. Biochem Biophys Res Commun 241:79-85

Gundert-Remy U, Bernauer U, Blömeke B, Döring B, Fabian E, Goebel C, Hessel S, Jäckh C, Lampen A, Oesch F, Petzinger E, Völkel W, Roos PH (2014) Extrahepatic metabolism at the body's internal-external interfaces. Drug Metab Rev 46:291-324. https://doi. org/10.3109/03602532.2014.900565

Hakkola J, Pelkonen O, Pasanen M, Raunio H (1998) Xenobioticmetabolizing cytochrome P450 enzymes in the human fetoplacental unit-role in intrauterine toxicity. Crit Rev Toxicol 28:35-72

Hall PM, Stupans I, Burgess W, Birkett DJ, McManus ME (1989) lmmunohistochemical localization of NADPH-cytochrome P-450 reductase in human tissues. Carcinogeneis 10:521-530

Han W, Pentecost BT, Pietropaolo RL, Fasco MJ, Spivack SD (2005) Estrogen receptor alpha increases basal and cigarette smoke extract-induced expression of CYP1A1 and CYP1B1, but not GSTP1, in normal human bronchial epithelial cells. Mol Carcinog 44:202-211

Hanlon N, Coldham N, Sauer MJ, Ioannides C (2009) Modulation of rat pulmonary carcinogen-metabolising enzyme systems by the isothiocyanates erucin and sulforaphane. Chem Biol Interact 177:115-120

Harrigan JA, McGarrigle BP, Sutter TR, Olson JR (2006) Tissue specific induction of cytochrome P450 (CYP) 1A1 and 1B1 in rat liver and lung following in vitro (tissue slice) and in vivo exposure to benzo(a)pyrene. Toxicol In Vitro 20:426-438

Hart SN, Li Y, Nakamoto K, Subileau EA, Steen D, Zhong XB (2010) A comparison of whole genome gene expression profiles of HepaRG cells and HepG2 cells to primary human hepatocytes and human liver tissues. Drug Metab Dispos 38:988-994

Hasegawa MM, Nishi Y, Tsuda H, Inui N, Morimoto K (1988) Effects of diesel exhaust particles on chromosome aberration, sister chromatid exchange and morphological transformation in cultured mammalian cells. Cancer Lett 42:61-66

Hazinski TA, France M, Kennedy KA, Hansen TN (1989) Cimetidine reduces hyperoxic lung injury in lambs. J Appl Physiol 67:2586-2592

He D, Frost AR, Falany CN (2005) Biochim Biophys Acta 1724:119-126

He X-Y, Tang L, Wang S-L, Cai Q-S, Wang J-S, Hong J-Y (2006) Efficient activation of aflatoxin B1 by cytochrome P450 2A13, an enzyme predominantly expressed in human respiratory tract. Int J Cancer 118:2665-2671

Hecht SS (1999) Tobacco smoke carcinogens and lung cancer. J Natl Cancer Inst 91:1194-1210

Hellmold H, Overvik E, Stromstedt M, Gustafsson JA (1993) Cytochrome $\mathrm{P} 450$ forms in the rodent lung involved in the metabolic activation of food-derived heterocyclic amines. Carcinogenesis 14:1751-1757

Her C, Szumlanski C, Aksoy IA, Weinshilboum RM (1996) Human jejunal estrogen sulfotransferase and dehydroepiandrosterone sulfotransferase immunochemical characterization of individual variation. Drug Metab Dispos 24:1328-1335

Hermanns MI, Fuchs S, Bock M et al (2009) Primary human coculture model of alveolo-capillary unit to study mechanisms of injury to peripheral lung. Cell Tissue Res 336:91-105. https://doi. org/10.1007/s00441-008-0750-1

Hermanns MI, Kasper J, Dubruel P et al (2010) An impaired alveolarcapillary barrier in vitro: Effect of proinflammatory cytokines and consequences on nanocarrier interaction. J R Soc Interface 7(Suppl 1):S41-S54. https://doi.org/10.1098/rsif.2009.0288. focus

Hess A, Wang-Lauenstein L, Braun A, Kolle SN, Landsiedel R, Liebsch M, Ma-Hock L, Pirow R, Schneider X, Steinfath M, Vogel S, Martin C, Sewald K (2016) Prevalidation of the ex vivo model PCLS for prediction of respiratory toxicity. Toxicol In Vitro 32:347-361. https://doi.org/10.1016/j.tiv.2016.01.006

Hoang ATN, Chen P, Juarez J et al (2012) Dendritic cell functional properties in a three-dimensional tissue model of human lung mucosa. Am J Physiol Lung Cell Mol Physio 302:L226-L237. https://doi.org/10.1152/ajplung.00059.2011

Hoet PH, Demedts M, Nemery B (1997) In vitro modulation of the $\mathrm{P} 450$ activities of hamster and human lung slices. Cell Biol Toxicol 13:185-192

Hofmann C, Pütz C, Semder B, Faller TH, Csanády GA, Filser JG (2006) Styrene-7,8-oxide burden in ventilated, perfused lungs of mice and rats exposed to vaporous styrene. Toxicol Sci 90:39-48

Hosseinpour F, Wikvall K (2000) Porcine microsomal vitamin D3 25-hydroxylase (CYP2D25): catalytic properties, tissue distribution, and comparison with human CYP2D6. J Biol Chem 44:34650-34655

Howie AF, Forrester LM, Glancey MJ, Schlager JJ, Powis G, Beckett GJ, Hayes JD, Wolf CR (1990) Glutathione $S$-transferase and glutathione peroxidase expression in normal and tumour human tissues. Carcinogenesis 11(3):451-458

Huber WW, McDaniel LP, Kaderlik KR, Teitel CH, Lang NP, Kadlubar FF (1997) Chemoprotection against the formation of colon DNA adducts from the food-borne carcinogen 
2-amino-1-methyl-6-phenylimidazo[4,5-b]-pyridine (PhIP) in the rat. Mutat Res 376:115-122

Huber WW, Prustomersky S, Delbanco E, Uhl M, Scharf G, Turesky RJ, Thier R, Schulte-Hermann R (2002) Enhancement of the chemoprotective enzymes glucuronosyl transferase and glutathione transferase in specific organs of the rat by the coffee components kahweol and cafestol. Arch Toxicol 76:209-217

Huh D, Matthews BD, Mammoto A et al (2010) Reconstituting organlevel lung functions on a chip. Science 328:1662-1668. https:// doi.org/10.1126/science.1188302

Huh D, Torisawa YS, Hamilton GA et al (2012) Microengineered physiological biomimicry: organs-on-chips. Lab Chip 12:2156-2164. https://doi.org/10.1039/c2lc40089h

Huijzer JC, Adams JD Jr, Jaw JY, Yost GS (1989) Inhibition of 3-methylindole bioactivation by the cytochrome P-450 suicide substrates 1-aminobenzotriazole and alpha-methylbenzylaminobenzotriazole. Drug Metab Dispos 17:37-42

Hukkanen J, Hakkola J, Anttila S, Piipari R, Karjalainen A, Pelkonen O, Raunio H (1997) Detection of mRNA encoding xenobioticmetabolizing cytochrome P450s in human bronchoalveolar macrophages and peripheral blood lymphocytes. Mol Carcinogen 20:224-230

Hukkanen J, Lassila A, Paivarinta K, Valanne S, Sarpo S et al (2000) Induction and regulation of xenobiotic-metabolizing cytochrome P450s in the human A549 lung adenocarcinoma cell line. Am J Respir Cell Mol Biol 22:360-366

Hukkanen J, Pelkonen O, Hakkola J, Raunio H (2002) Expression and regulation of xenobiotic-metabolizing cytochrome P450 (CYP) enzymes in human lung. Crit Rev Toxicol 32:391-411

Hukkanen J, Vaisanen T, Lassila A et al (2003) Regulation of CYP3A5 by glucocorticoids and cigarette smoke in human lung-derived cells. J Pharmacol Exp Ther 304:745-752

Hume R, Barker EV, Coughtrie MW (1996) Differential expression and immunohistochemical localisation of the phenol and hydroxysteroid sulphotransferase enzyme families in the developing lung. Histochem Cell Biol 105:147-152

Hynes DE, DeNicola DB, Carlson GP (1999) Metabolism of styrene by mouse and rat isolated lung cells. Toxicol Sci 51:195-201

Iba MM, Scholl H, Fung J, Thomas PE, Alam J (1998) Induction of pulmonary CYP1A1 by nicotine. Xenobiotica 28:827-843

Iba MM, Shin M, Caccavale RJ (2010) Cytochromes P4501 (CYP1): catalytic activities and inducibility by diesel exhaust particle extract and benzo[a]pyrene in intact human lung ex vivo. Toxicology 273:35-44. https://doi.org/10.1016/j.tox.2010.04.012

Igarashi K, Kitajima S, Aisaki K, Tanemura K, Taquahashi Y, Moriyama N, Ikeno E, Matsuda N, Saga Y, Blumberg B, Kanno J (2012) Development of humanized steroid and xenobiotic receptor mouse by homologous knock-in of the human steroid and xenobiotic receptor ligand binding domain sequence. J Toxicol Sci 37:373-380

Imaoka S, Hiroi T, Tamura Y, Yamazaki H, Shimada T, Komori M et al (1995) Mutagenic activation of 3-methoxy-4-aminoazobenzene by mouse renal cytochrome P450 CYP4B1: cloning and characterization of mouse CYP4B1. Arch Biochem Biophys 321:255-262

Ioannides C (2013) Up-regulation of cytochrome P450 and phase II enzymes by xenobiotics in precision-cut tissue slices. Xenobiotica 43:15-28. https://doi.org/10.3109/00498254.2012.698766

Iskandar AR, Xiang Y, Frentzel S, Talikka M, Leroy P, Kuehn D, Guedj E, Martin F, Mathis C, Ivanov NV, Peitsch MC, Hoeng J (2015) Impact assessment of cigarette smoke exposure on organotypic bronchial epithelial tissue cultures: a comparison of mono-culture and coculture model containing fibroblasts. Toxicol Sci 147:207-221. https://doi.org/10.1093/toxsci/kfv122
Iwanari M, Nakajima M, Ryoichi K et al (2002) Induction of CYO1A1, CYP1A2, and CYP1B1 mRNAs by nitropoly-cyclic aromatic hydrocarbons in various human tissue-derived cells: chemical-, cytochrome P450 isoform-, and cell-specific differences. Arch Toxicol 76:287-298

Jiang H, Vudathala DK, Blair IA, Penning TM (2006) Competing roles of aldo-keto reductase 1A1 and cytochrome P4501B1 in benzo[a]pyrene-7,8-diol activation in human bronchoalveolar H358 cells: role of AKRs in P4501B1 induction. Chem Res Toxicol 19:68-78

Jiang W, Wang L, Zhang W, Coffee R, Fazili IS, Moorthy B (2009) Persistent induction of cytochrome P450 (CYP)1A enzymes by 3 -methylcholanthrene in vivo in mice is mediated by sustained transcriptional activation of the corresponding promoters. Biochem Biophys Res Commun 390:1419-1424. https://doi. org/10.1016/j.bbrc.2009.11.021

Jin B, Park DW, Nam K-W, Goo Taeg O, Lee Y-S, Ryu D-Y (2004) CpG methylation of the mouse CYP1A2 promoter. Toxicol Lett 152:11-18

Johanson G, Ernstgard L, Gullstrand E, Lof A, Osterman-Golkar S, Williams CC, Sumner SCJ (2000) Styrene oxide in blood, hemoglobin adducts, and urinary metabolites in human volunteers exposed to ${ }^{13} \mathrm{C} 8$-styrene vapors. Toxicol Appl Pharmacol 168:36-49

Jones KG, Holland JF, Fo Ureman GL, Bend JR, Fouts JR (1983) Xenobiotic metabolism in Clara cells and alveolar type II cells isolated from lungs of rats treated with $\beta$-naphthoflavone. Pharmacol Exp Therap 225:316-319

Juricek L, Bui LC, Busi F, Pierre S, Guyot E, Lamouri A, Dupret JM, Barouki R, Coumoul X, Rodrigues-Lima F (2015) Activation of the aryl hydrocarbon receptor by carcinogenic aromatic amines and modulatory effects of their $N$-acetylated metabolites. Arch Toxicol 89:2403-2412. https://doi.org/10.1007/s0020 4-014-1367-7

Kalabus JL, Cheng Q, Jamil RG, Schuetz EG, Blanco JG (2012) Induction of carbonyl reductase 1 (CBR1) expression in human lung tissues and lung cancer cells by the cigarette smoke constituent benzo[a]pyrene. Toxicol Lett 21:266-273. https://doi. org/10.1016/j.toxlet.2012.04.006

Kalpana Deepa Priya D, Gayathri R, Sakthisekaran D (2011) Role of sulforaphane in the anti-initiating mechanism of lung carcinogenesis in vivo by modulating the metabolic activation and detoxification of benzo(a)pyrene. Biomed Pharmacother 65:9-16. https ://doi.org/10.1016/j.biopha.2010.08.009

Karlgren M, Miura S, Ingelman-Sundberg M (2005) Novel extrahepatic cytochrome P450s. Toxicol Appl Pharmacol 207(Suppl):57-61

Kasper J, Hermanns MI, Bantz C, Maskos M, Stauber R, Pohl C, Unger RE, Kirkpatrick JC (2011) Inflammatory and cytotoxic responses of an alveolar-capillary coculture model to silica nanoparticles: comparison with conventional monocultures. Part Fibre Toxicol 8:6. https://doi.org/10.1186/1743-8977-8-6

Kastelova A, Dimova S, Nemery B (2003) Effects of propranolol on xenobiotic enzyme activities in rat type II pneumocytes and alveolar macrophages in vivo. Methods Find Exp Clin Pharmacol 25:797-802

Kaufmann W, Mellert W, van Ravenzwaay B, Landsiedel R, Poole A (2005) Effects of styrene and its metabolites on different lung compartments of the mouse-cell proliferation and histomorphology. Regul Toxicol Pharmacol 42:24-36

Keith IM, Olson EB Jr, Wilson NM, Jefcoate CR (1987) Immunological identification and effects of 3-methylcholanthrene and phenobarbital on rat pulmonary cytochrome P-450. Cancer Res 47:1878-1882

Kelly JD, Eaton DL, Guengerich FP, Coulombe RA (1997) Aflatoxin B1 activation in human lung. Toxicol Appl Pharmacol 144:88-95 
Keserü B, Barbosa-Sicard E, Popp R, Fisslthaler B, Dietrich A, Gudermann T, Hammock BD, Falck JR, Weissmann N, Busse R, Fleming I (2008) Epoxyeicosatrienoic acids and the soluble epoxide hydrolase are determinants of pulmonary artery pressure and the acute hypoxic pulmonary vasoconstrictor response. FASEB J 22:4306-4315. https://doi.org/10.1096/fj.08-112821

Kim JH, Sherman ME, Curriero FC, Guengerich FP, Strickland PT, Sutter TR (2004) Expression of cytochromes P450 1A1 and 1B1 in human lung from smokers, non-smokers, and ex-smokers. Toxicol Appl Pharmacol 199:210-219

Kivisto KT, Fritz P, Linder A, Friedel G, Beaune P, Kroemer HK (1995) Immunohistochemical localization of cytochromeP450 $3 \mathrm{~A}$ in human pulmonary carcinomas and normal bronchial tissue. Histochemistry 103:25-29

Klein SG, Hennen J, Serchi T, Blömeke B, Gutleb AC (2011) Potential of coculture in vitro models to study inflammatory and sensitizing effects of particles on the lung. Toxicol In Vitro 25:15161534. https://doi.org/10.1016/j.tiv.2011.09.006

Klein SG, Serchi T, Hoffmann L, Blömeke B, Gutleb AC (2013) An improved 3D tetraculture system mimicking the cellular organisation at the alveolar barrier to study the potential toxic effects of particles on the lung. Part Fibre Toxicol 10:31. https://doi. org/10.1186/1743-8977-10-31

Kletting S, Barthold S, Repnik U, Griffiths G, Loretz B, SchneiderDaum N, de Souza C-WC, Lehr CM (2018) Co-culture of human alveolar epithelial (hAELVi) and macrophage (THP-1) cell lines. Altex 35:211-222. https://doi.org/10.14573/altex.1607191

Koban R, Neumann M, Daugs A, Bloch O, Nitsche A, Langhammer S, Ellerbrok H (2018) A novel three-dimensional cell culture method enhances antiviral drug screening in primary human cells. Antiviral Res 150:20-29. https://doi.org/10.1016/j.antiv iral.2017.12.005

Köhler CU, Welge P, Roos PH (2006a) Regulation of CYP2E1 at the transcript level in minipig tissues. Naunyn-Schmiedeberg's Arch Pharmacol Mainz 372(Suppl. 1):95 (abstr. no 353)

Köhler CU, Welge P, Roos PH (2006b) Regulation of CYP2E1 at the protein and activity level in minipigs. Naunyn-Schmiedeberg's Arch Pharmacol Mainz 375(Suppl. 1):72 (abstr. no 347)

Konsue N, Ioannides C (2008) Tissue differences in the modulation of rat cytochromes $\mathrm{P} 450$ and phase II conjugation systems by dietary doses of phenethyl isothiocyanate. Food Chem Toxicol 46:3677-3683. https://doi.org/10.1016/j.fct.2008.09.046

Krueger SK, Yueh MF, Martin SR, Pereira CB, Williams DE (2001) Characterization of expressed full-length and truncated FMO2 from rhesus monkey. Drug Metab Dispos 29:693-700

Kurogi K, Chen M, Lee Y, Shi B, Yan T, Liu M-Y, Sakakibara Y, Suiko M, Liu M-C (2012) Sulfation of buprenorphine, pentazocine, and naloxone by human cytosolic sulfotransferases. Drug Metab Lett 6:109-115

Kuzuya Y, Adachi T, Hara H, Anan A, Izuhara K, Nagai H (2004) Induction of drug-metabolizing enzymes and transporters in human bronchial epithelial cells by beclomethasone dipropionate. IUBMB Life 56:355-359

Lake BG, Meredith C, Scott MP, Renwick AB, Price RJ (2003) Use of cultured precision-cut rat lung slices to study the in vitro induction of pulmonary cytochrome P450 forms. Xenobiotica 33:691-702

Landsiedel R, Fabian E, Tralau T, Luch A (2011) Chemical toxicity testing in vitro using cytochrome $\mathrm{P} 450$-expressing cell lines, such as human CYP1B1. Nat Protoc 6:677-688

Lanza DL, Code E, Crespi CL, Gonzalez FJ, Yost GS (1999) Specific dehydrogenation of 3-methylindole and epoxidation of naphthalene by recombinant human CYP2F1 expressed in lymphoblastoid cells. Drug Metab Dispos 27:798-803
Leclerc J, Courcot-Ngoubo Ngangue E, Cauffiez C, Allorge D, Pottier N, Lafitte JJ, Debaert M, Jaillard S, Broly F, Lo-Guidice JM (2011) Xenobiotic metabolism and disposition in human lung: transcript profiling in non-tumoral and tumoral tissues. Biochimie 93:1012-1027. https://doi.org/10.1016/j.bioch i.2011.02.012

Lehmann AD, Daum N, Bur M et al (2011) Anin vitrotriple cell coculture model with primary cells mimicking the human alveolar epithelial barrier. Eur J Pharmaceut Biopharmaceut 77:398-406. https://doi.org/10.1016/j.ejpb.2010.10.014

Lepers C, André V, Dergham M, Billet S, Verdin A, Garçon G, Dewaele D, Cazier F, Sichel F, Shirali P (2014) Xenobiotic metabolism induction and bulky DNA adducts generated by particulate matter pollution in BEAS-2B cell line: geographical and seasonal influence. J Appl Toxicol 34:703-713. https://doi.org/10.1002/ jat.2931 (Epub 2013 Sep 30)

Levin W, Lu AY, Thomas PE, Ryan D, Kizer DE, Griffin MJ (1978) Identification of epoxide hydrase as the preneoplastic antigen in rat liver hyperplastic nodules. Proc Natl Acad Sci USA 75:3240-3243

Lewis DFV, Ito Y, Lake BG (2009) Molecular modeling of CYP2F substrates: comparison of naphthalene metabolism by human, rat and mouse CYP2F subfamily enzymes. Drug Metabol Drug Interact 20:229-257

Li Q, Csanády GA, Kessler W, Klein D, Pankratz H, Pütz C, Richter N, Filser JG (2011a) Kinetics of ethylene and ethylene oxide in subcellular fractions of lungs and livers of male B6C3F1 mice and male fischer 344 rats and of human livers. Toxicol Sci 123:384-398. https://doi.org/10.1093/toxsci/kfr194

Li L, Wei Y, Van Winkle L, Zhang QY, Zhou X, Hu J, Xie F, Kluetzman K, Ding X (2011b) Generation and characterization of a Cyp2f2-null mouse and studies on the role of CYP2F2 in naphthalene-induced toxicity in the lung and nasal olfactory mucosa. J Pharmacol Exp Ther 339:62-71. https://doi.org/10.1124/ jpet.111.184671

Libalova H, Rossner P, Vrbova K, Brzicova T, Sikorova J, VojtisekLom M, Beranek V, Klema J, Ciganek M, Neca J, Pencikova K, Machala M, Topinka J (2016) Comparative analysis of toxic responses of organic extracts from diesel and selected alternative fuels engine emissions in human lung BEAS-2B cells. Int $\mathrm{J}$ Mol Sci 17:E1833

Líbalová H, Krčková S, Uhlîrová K, Kléma J, Ciganek M, Rössner P Jr, Šrám RJ, Vondráček J, Machala M, Topinka J (2014) Analysis of gene expression changes in A549 cells induced by organic compounds from respirable air particles. Mutat Res 770:94-105. https://doi.org/10.1016/j.mrfmmm.2014.10.002

Lieber M, Smith B, Szakal A et al (1976) A continuous tumor-cell line from a human lung carcinoma with properties of type II alveolar epithelial cells. Int J Cancer 17:62-70

Lin HR, Wu CC, Wu YH, Hsu CW, Cheng ML, Chiu DT (2013) Proteome-wide dysregulation by glucose-6-phosphate dehydrogenase (G6PD) reveals a novel protective role for G6PD in aflatoxin $\mathrm{B}_{1}$-mediated cytotoxicity. J Proteome Res 12:3434-3448

Lindsay J, Wang LL, Li Y, Zhou SF (2008) Structure, function and polymorphism of human cytosolic sulfotransferases. Curr Drug Metab 9:99-105

Ling G, Wei Y, Ding X (2007) Transcriptional regulation of human CYP2A13 expression in the respiratory tract by CCAAT/ enhancer binding protein and epigenetic modulation. Mol Pharmacol 71:807-816

Longhin E, Capasso L, Battaglia C, Proverbio MC, Cosentino C, Cifola I, Mangano E, Camatini M, Gualtieri M (2016) Integrative transcriptomic and protein analysis of human bronchial BEAS-2B exposed to seasonal urban particulate matter. Environ Pollut 209:87-98 
Lorenz J, Schmassmann H, Ohnhaus E, Oesch F (1979) Activities of polycyclic hydrocarbon activating and inactivating enzymes in human lungs of smokers, non-smokers, lung-cancer and noncancer patients. Arch Toxicol 1979(Suppl 2):483-489

Lorenz J, Glatt HR, Fleischmann R, Ferlinz R, Oesch F (1984) Drug metabolism in man and its relationship to that in three rodent species: monooxygenase, epoxide hydrolase, and glutathione $S$-transferase activities in subcellular fractions of lung and liver. Biochem Med 32:43-56

Ma JY, Yang HM, Barger MW, Siegel PD, Zhong BZ, Kriech AJ, Castranova V (2002) Alteration of pulmonary cytochrome p-450 system: effects of asphalt fume condensate exposure. J Toxicol Environ Health A 65:1247-1260

Mace K, Vautravers P, Granato D et al (1997) Development of CYP450-expressing human bronchial epithelial cell lines for in vitro pharmacotoxicologic application. Vitro Toxicol 10:85-92

Macé K, Bowman ED, Vautravers P, Shields PG, Harris CC, Pfeifer AMA (1998) Characterisation of xenobiotic-metabolising enzyme expression in human bronchial mucosa and peripheral lung tissues. Eur J Cancer 34:914-920

Magdaleno SM, Wang G, Jackson KJ, Ray MK, Welty S, Costa RH, DeMayo FJ (1997) Interferon-gamma regulation of Clara cell gene expression: in vivo and in vitro. Am J Physiol 272:L1142-L1151

Mainwaring GW, Williams SM, Foster JR, Tugwood J, Green T (1996) The distribution of theta-class glutathione $S$-transferases in the liver and lung of mouse, rat and human. Biochem J. 318(Pt 1):297-303

Mansour H, Levacher M, Azoulay-Dupuis E, Moreau J, Marquetty C, Gougerot-Pocidalo MA (1988) Genetic differences in response to pulmonary cytochrome P-450 inducers and oxygen toxicity. J Appl Physiol 64:1376-1381

Mathia NR, Timoszyk J, Stetsko PI et al (2002) Permeability characteristics of calu-3 human bronchial epithelial cells: in vitro-in vivo correlation to predict lung absorption in rats. J Drug Target $10: 31-40$

McLellan LI, Harrison DJ, Hayes JD (1992) Modulation of glutathione S-transferases and glutathione peroxidase by the anticarcinogen butylated hydroxyanisole in murine extrahepatic organs. Carcinogenesis 13:2255-2261

McLemore TL, Adelberg S, Liu MC, McMahon NA, Yu SJ, Hubbard WC, Czerwinski M, Wood TG, Storeng R, Lubet RA, Eggleston JC, Boyd MR, Hines RN (1990) Expression of CYP1A1 gene in patients with lung cancer: evidence for cigarette smoke-induced gene expression in normal lung tissue and for altered gene regulation in primary pulmonary carcinomas. J Natl Cancer Inst 82:1333-1339

McManus ME, Boobis AR, Pacifici GM, Frempong RY, Brodie MJ, Kahn GC, Whyte C, Davies DS (1980) Xenobiotic metabolism in the human lung. Life Sci 11(26):481-487

Mendrala AL, Langvardt PW, Nitschke KD, Quast JF, Nolan RJ (1993) In vitro kinetics of styrene and styrene oxide metabolism in rat, mouse and human. Arch Toxicol 67:18-27

Messina A, Chirulli V, Gervasi PG, Longo V (2008) Purification, molecular cloning, heterologous expression and characterization of pig CYP1A2. Xenobiotica 38:1453-1470

Messina A, Nannelli A, Fiorio R, Longo V, Gervasi PG (2009) Expression and inducibility of CYP1A1, 1A2, 1B1 by -naphthoflavone and CYP2B22, 3A22, 3A29 3A46 by rifampicin in the respiratory and olfactory mucosa of pig. Toxicology 260:47-52

Miller VL, Lin HK, Murugan P, Fan M, Penning TM, Brame LS, Yang Q, Fung KM (2012) Aldo-keto reductase family 1 member C3 (AKR1C3) is expressed in adenocarcinoma and squamous cell carcinoma but not small cell carcinoma. Int J Clin Exp Pathol $5: 278-89$
Mollerup S, Ryberg D, Hewer A, Phillips DH, Haugen A (1999) Sex differences in lung CYP1A1 expression and DNA adduct levels among lung cancer patients. Cancer Res 59:3317-3320

Mollerup S, Øvrebø S, Haugen A (2001) Lung carcinogenesis: resveratrol modulates the expression of genes involved in the metabolism of PAH in human bronchial epithelial cells. Int J Cancer 92:18-25

Mollerup S, Berge G, Bære R, Skaug V, Hewer A, Phillips DH, Stangeland L, Haugen A (2006) Sex differences in risk of lung cancer: expression of genes in the PAH bioactivation pathway in relation to smoking and bulky DNA adducts. Int J Cancer 119:741-744

Nagata K, Martin BM, Gillette JR, Sasame HA (1990) Isozymes of cytochrome P-450 that metabolize naphthalene in liver and lung of untreated mice. Drug Metab Dispos 18:557-564

Nakajima T, Elovaara E, Gonzalez FJ, Gelboin HV, Raunio H, Pelkonen O, Vainio H, Aoyama T (1994) Styrene metabolism by cDNA-expressed human hepatic and pulmonary cytochromes P450. Chem Res Toxicol 7:891-896

Nakanishi M, Deyashiki Y, Ohshima K, Hara A (1995) Cloning, expression and tissue distribution of mouse tetrameric carbonyl reductase. Identity with an adipocyte $27-\mathrm{kDa}$ protein. Eur J Biochem 228:381-387

Nakayama Wong LS, Aung HH, Lamé MW, Wegesser TC, Wilson DW (2011) Fine particulate matter from urban ambient and wildfire sources from California's San Joaquin Valley initiate differential inflammatory, oxidative stress, and xenobiotic responses in human bronchial epithelial cells. Toxicol In Vitro 25:1895-1905. https://doi.org/10.1016/j.tiv.2011.06.001

Nakamura A, Nakajima M, Yamanaka H et al (2008a) Expression of UGT1A and UGT2B mRNA in human normal tissues and various cell lines. Drug Metab Dispos 36:1461-1464

Nakamura T, Yamamori M, Sakaeda T (2008b) Pharmacogenetics of intestinal absorption. Curr Drug Deliv 5:153-169

Nannelli A, Chirulli V, Longo V, Gervasi PG (2008) Expression and induction by rifampicin of CAR- and PXR-regulated CYP2B and CYP3A in liver, kidney and airways of pig. Toxicology 252:105-112

Nave R, Fisher R, Zech K (2006) In vitro metabolism of ciclesonide in human lung and liver precision-cut tissue slices. Biopharm Drug Dispos 27:197-207

Newland N, Baxter A, Hewitt K, Minet E (2011) CYP1A1/1B1 and CYP2A6/2A13 activity is conserved in cultures of differentiated primary human tracheobronchial epithelial cells. Toxicol In Vitro 25:922-929. https://doi.org/10.1016/j.tiv.2011.02.014

Nhamburo PT, Gonzalez FJ, McBride OW, Gelboin HV, Kimura S (1989) Identification of a new P450 expressed in human lung: complete cDNA sequence, cDNA-directed expression, and chromosome mapping. Biochemistry 28:8060-8066

Nhamburo PT, Kimura S, McBride OW, Kozak CA, Gelboin HV, Gonzalez FJ (1990) The human CYP2F gene subfamily: identification of a cDNA encoding a new cytochrome P450, cDNAdirected expression, and chromosome mapping. Biochemistry 29:5491-5499

Nishimura M, Naito S (2006) Tissue-specific mRNA expression profiles of human phase I metabolizing enzymes except for cytochrome P450 and phase II metabolizing enzymes. Drug Metab Pharmacokinet 21:357-374

Oesch F, Bentley P (1976) Antibodies against homogeneous epoxide hydratase: evidence for a single enzyme hydrating styrene oxide and benzo[a]pyrene 4,5-(K-region-)oxide. Nature 259:53-55

Oesch F, Jerina DM, Daly J (1971) A radiometric assay for hepatic epoxide hydrase activity with 7-3H-styrene oxide. Biochim Biophys Acta 227:685-691

Oesch F, Glatt HR, Schmassmann HU (1977) The apparent ubiquity of epoxide hydratase in rat organs. Biochem Pharmacol 26:603-607 
Oesch R, Schmassmann H, Ohnhaus E, Althaus U, Lorenz J (1980) Monooxygenase, epoxide hydrolase, and glutathione- $S$-transferase activities in human lung. Variation between groups of bronchogenic carcinoma and non-cancer patients. Carcinogenesis 1:827-835

Oesch F, Milbert U, Friedberg T, Wolf CR (1983) Identification of novel glutathione $S$-transferases in kidney and lung and the inducibility of various isozymes in liver and other organs. In: Rydström J, Montelius I, Bengtsson M (eds) Extrahepatic drug metabolism and chemical carcinogenesis. Elsevier, New York, pp 163-170

Oesch F, Schladt L, Hartmann R, Timms C, Wörner W (1986) Rat cytosolic epoxide hydrolase. Adv Exp Med Biol 197:195-201

Oesch-Bartlomowicz B, Oesch F (2007) Mechanisms of toxification and detoxification which challenge drug candidates and drugs. In: Testa B, van de Waterbeemd $\mathrm{H}$ (eds) Comprehensive medicinal chemistry. Elsevier, Oxford, pp 193-214

Okamatsu G, Komatsu T, Ono Y, Inoue H, Uchide T, Onaga T, Endoh D, Kitazawa T, Hiraga T, Uno Y, Teraoka H (2017) Characterization of feline cytochrome P450 2B6. Xenobiotica 47:93-102. https://doi.org/10.3109/00498254.2016.1145754

Olsavsky KM, Page JL, Johnson MC, Zarbl H, Strom SC, Omiecinski CJ (2007) Gene expression profiling and differentiation assessment in primary human hepatocyte cultures, established hepatoma cell lines, and human liver tissues. Toxicol Appl Pharmacol 222:42-56

Omiecinski CJ, Redlich CA, Costa P (1990) Induction and developmental expression of cytochrome P450IA1 messenger RNA in rat and human tissues: detection by the polymerase chain reaction. Cancer Res 50:4315-4321

Omiecinski CJ, Aicher L, Holubkov R, Checkoway H (1993) Human peripheral lymphocytes as indicators of microsomal epoxide hydrolase activity in liver and lung. Pharmacogenetics 3:150-158

Omiecinski CJ, Aicher L, Swenson L (1994) Developmental expression of human microsomal epoxide hydrolase. J Pharmacol Exp Ther 269:417-423

Overby L, Nishio SJ, Lawton MP, Plopper CG, Philpot RM (1992) Cellular localization of flavin-containing monooxygenase in rabbit lung. Exp Lung Res 18:131-144

Pacifici GM, Bencini C, Rane A (1986) Acetyltransferase in humans: development and tissue distribution. Pharmacology 32:283-291

Pacifici GM, Temellini A, Giuliani L, Rane A, Thomas H, Oesch F (1988a) Cytosolic epoxide hydrolase in humans: development and tissue distribution. Arch Toxicol 62:254-257

Pacifici GM, Franchi M, Bencini C, Repetti F, Di Lascio N, Muraro GB (1988b) Tissue distribution of drug-metabolizing enzymes in humans. Xenobiotica 18:849-856

Palackal NT, Lee SH, Harvey RG, Blair IA, Penning TM (2002) Activation of polycyclic aromatic hydrocarbon trans-dihydrodiol proximate carcinogens by human aldo-keto reductase (AKR1C) enzymes and their functional overexpression in human lung carcinoma (A549) cells. J Biol Chem 277:24799-24808

Paolini M, Mesirca R, Pozzetti L, Sapone A, Cantelli-Forti G (1995) Induction of CYP2B1 mediated pentoxyresorufin O-dealkylase activity in different species, sex and tissue by prototype 2B1-inducers. Chem Biol Interact 95:127-139

Pavek P, Dvorak Z (2008) Xenobiotic-induced transcriptional regulation of xenobiotic metabolizing enzymes of the cytochrome P450 superfamily in human extrahepatic tissues. Curr Drug Metab 9:129-143

Petruzzelli S, Bernard P, Paoletti P, Rane A, Giuntini C, Pacifici GM (1988) Presence of epoxide hydrolase and glutathione S-transferase in human pulmonary alveolar macrophages. Eur J Clin Pharmacol 34:419-421
Pettigrew NE, Brush EJ, Roberta F (2001) 3-Methyleneoxindole: an affinity label of glutathione s-transferase pi which targets Tryptophan 38. Biochemistry 40:7549-7558

Pezzulo AA, Starner TD, Scheetz TE et al (2010) The air-liquid interface and use of primary cell cultures are important to recapitulate the transcriptional profile of in vivo airway epithelia. Am J Physiol Lung Cell Mol Physiol 300:L25-L31

Pfaffli P, Hesso A, Vainio H, Hirvonen M (1981) 4-Vinylphenol excretion suggestive of arene oxide formation in workers occupationally exposed to styrene. Toxicol Appl Pharmacol 60:85-90

Piipari R, Savela K, Nurminen T, Hukkanen J, Raunio H, Hakkola J, Mäntylä T, Beaune P, Edwards RJ, Boobis AR, Anttila S (2000) Expression of CYP1A1, CYP1B1 and CYP3A, and polycyclic aromatic hydrocarbon-DNA adduct formation in bronchoalveolar macrophages of smokers and non-smokers. Int J Cancer 86:610-616

Plopper CG, Cranz DL, Kemp L, Serabjit-Singh CJ, Philpot RM (1987) Immunohistochemical demonstration of cytochrome P-450 monooxygenase in Clara cells throughout the tracheobronchial airways of the rabbit. Exp Lung Res 13:59-68

Poch MT, Cutler NS, Yost GS, Hines RN (2005) Molecular mechanisms regulating human CYP4B1 lung-selective expression. Drug Metab Dispos 33:1174-1184

Ponomarkov V, Tomatis L (1978) Effects of long-term oral administration of styrene to rats and mice. Scand J Work Environ Health 4(suppl. 2):127-135

Port JL, Yamaguchi K, Du B, De Lorenzo M, Chang M, Heerdt PM, Kopelovich L, Marcus CB, Altorki NK, Subbaramaiah K, Dannenberg AJ (2004) Tobacco smoke induces CYP1B1 in the aerodigestive tract. Carcinogenesis 25:2275-2281

Price RJ, Renwick AB, Beamand JA, Esclangon F, Wield PT, WaltersDG Lake BG (1995) Comparison of the metabolism of 7-ethoxycoumarin and coumarin in precision-cut rat liver and lung slices. Food Chem Toxicol 33:233-237

Price RJ, Walters DG, Hoff C, Mistry H, Renwick AB, Wield PT, Beamand JA, Lake BG (1996) Metabolism of [ring-U-14C] agaritine by precision-cut rat, mouse and human liver and lung slices. Food Chem Toxicol 34:603-609

Pushparajah DS, Umachandran M, Plant KE, Plant N, Ioannides C (2007) Evaluation of the precision-cut liver and lung slice systems for the study of induction of CYP1, epoxide hydrolase and glutathione $S$-transferase activities. Toxicology 231:68-80

Pushparajah DS, Umachandran M, Plant KE, Plant N, Ioannides C (2008a) Differential response of human and rat epoxide hydrolase to polycyclic aromatic hydrocarbon exposure: studies using precision-cut tissue slices. Mutat Res 640:153-161. https://doi.org/10.1016/j.mrfmmm.2008.01.004

Pushparajah DS, Umachandran M, Plant KE, Plant N, Ioannides C (2008b) Up-regulation of the glutathione $S$-transferase system in human liver by polycyclic aromatic hydrocarbons; comparison with rat liver and lung. Mutagenesis 23:299-308. https:// doi.org/10.1093/mutage/gen012

Ramirez RD, Sheridan S, Girard L et al (2004) Immmortalization of human bronchial epithelial cells in the absence of viral oncoproteins. Cancer Res 64:9027-9034

Ramakanth S, Thornton-Manning JR, Wang H, Maxwell H, Yost GS (1994) Correlation between pulmonary cytochrome P450 transcripts and the organselective pneumotoxicity of 3-methylindole. Toxicol Lett 71:77-85

Raunio H, Hakkola J, Hukkanen J, Pelkonen O, Edwards RJ, Boobis A, Anttila S (1998) Expression of xenobiotic-metabolizing cytochrome P450s in human pulmonary tissues. Arch Toxicol 20:465-469

Raunio H, Hakkola J, Hukkanen J et al (1999) Expression of xenobiotic-metabolizing CYPs in human pulmonary tissue. Exp Toxicol Pathol 51:412-417 
Raunio H, Hakkola J, Pelkonen O (2004) Regulation of CYP3A genes in the human respiratory tract. Chem Biol Interact 151:53-62

Reddel RR, Ke Y, Gerwin BI et al (1988) Transformation of human bronchial epithelial cells by infection with SV40 or adenovirus-12 SV40 hybrid virus, or transfection via strontium phosphate coprecipitation with a plasmid containing SV40 early region genes. Cancer Res 48:1904-1909

Reddel RR, Salghetti SE, Willey JC et al (1993) Development of tumorigenicity in simian virus 40- immortalized human bronchial epithelial cell lines. Cancer Res 53:985-991

Ren Q, Murphy SE, Zheng Z, Lazarus P (2000) $O$-Glucuronidation of the lung carcinogen 4-(methylnitrosamino)-1-(3-pyridyl)-1butanol (NNAL) by human UDP-glucuronosyltransferases 2B7 and 1A9. Drug Metab Dispos 28:1352-1360

Renaud HJ, Cui JY, Khan M, Klaassen CD (2011) Tissue distribution and gender-divergent expression of 78 cytochrome P450 mRNAs in mice. Toxicol Sci 124:261-277. https://doi.org/10.1093/toxsc $\mathrm{i} / \mathrm{kfr} 240$

Richard K, Hume R, Kaptein E, Stanley EL, Visser TJ, Coughtrie MW (2001) Sulfation of thyroid hormone and dopamine during human development: ontogeny of phenol sulfotransferases and arylsulfatase in liver, lung, and brain. J Clin Endocrinol Metab 86:2734-2742

Rioux N, Castonguay A (1998) Prevention of NNK-induced lung tumorigenesis in A/J mice by acetylsalicylic acid and NS-398. Cancer Res 58:5354-5360

Rivera SP, Saarikoski ST, Hankinson O (2002) Identification of a novel dioxin-inducible cytochrome P450. Mol Pharmacol 61:255-259

Robertson LG, Philpot RM, Zeiger E, Wolf CR (1981) Specificity of rabbit pulmonary cytochrome $\mathrm{P}-450$ isozymes in the activation of several aromatic amines and aflatoxin B1. Mol Pharmacol 20:662-668

Robertson IG, Jensson H, Mannervik B, Jernström B (1986) Glutathione transferases in rat lung: the presence of transferase 7-7, highly efficient in the conjugation of glutathione with the carcinogenic (+)-7 beta, 8 alpha-dihydroxy-9 alpha, 10 alpha-oxy7,8,9,10-tetrahydrobenzo[a]pyrene. Carcinogenesis 7:295-299

Roos PH, Tschirbs S, Welge P, Hack A, Theegarten D, Mogilevski G, Wilhelm M (2002) Induction of cytochrome P450 1A1 in multiple organs of minipigs after oral exposure to soils contaminated with polycyclic aromatic hydrocarbons (PAH). Arch Toxicol 76:326-334

Rothbauer M, Rosser JM, Zirath H, Ertl P (2019) Tomorrow today: organ-on-a-chip advances towards clinically relevant pharmaceutical and medical in vitro models. Curr Opin Biotechnol $55: 81-86$

Rothen-Rutishauser BM, Kiama SG, Gehr P (2005) A three-dimensional cellular model of the human respiratory tract to study the interaction with particles. Am J Respir Cell Mol Biol 32:281-289

Rothen-Rutishauser B, Blank F, Muhlfeld C et al (2008) In vitro models of the human epithelial airway barrier to study the toxic potential of particulate matter. Expert Opin Drug Metab Toxicol 4:1075-1089

Ruangyuttikarn W, Appleton ML, Yost GS (1991) Metabolism of 3-methylindole in human tissues. Drug Metab Dispos 19:977-984

Runge DM, Stock TW, Lehmann T, Taege C, Bernauer U, Stolz DB, Hofmann S, Foth H (2001) Expression of cytochrome P450 2E1 in normal human bronchial epithelial cells and activation by ethanol in culture. Arch Toxicol 75:335-345

Rylander T, Neve EP, Ingelman-Sundberg M, Oscarson M (2001) Identification and tissue distribution of the novel human cytochrome P450 2S1 (CYP2S1). Biochem Biophys Res Commun 281:529-535
Rynning I, Neca J, Vrbova K, Libalova H, Rossner P Jr, Holme JA, Gützkow KB, Afanou AKJ, Arnoldussen YJ, Hruba E, Skare Ø, Haugen A, Topinka J, Machala M, Mollerup S (2018) In vitro transformation of human bronchial epithelial cells by diesel exhaust particles: gene expression profiling and early toxic responses. Toxicol Sci 166:51-64

Saarikoski ST, Husgafvel-Pursiainen K, Hirvonen A, Vainio H, Gonzalez FJ, Anttila S (1998) Localization of CYP1A1 mRNA in human lung by in situ hybridization: comparison with immunohistochemical findings. Int J Cancer 77:33-39

Saarikoski ST, Wikman HA, Smith G, Wolff CH, Husgafvel-Pursiainen K (2005) Localization of cytochrome P450 CYPS2S1 expression in human tissues by in situ hybridization and immunohistochemistry. J Histochem Cytochem 53:549-556

Sabourin PJ, Tynes RE, Philpot RM, Winquist S, Dahl AR (1988) Distribution of microsomal monooxygenases in the rabbit respiratory tract. Drug Metab Dispos 16:557-562

Sadler NC, Webb-Robertson BM, Clauss TR, Pounds JG, Corley R, Wright AT (2018) High-fat diets alter the modulatory effects of xenobiotics on cytochrome P450 activities. Chem Res Toxicol 31:308-318. https://doi.org/10.1021/acs.chemrestox.8b00008

Sainkhuu B, Park BS, Kim HW (2016) Induction of flavin-containing monooxygenase in mice by oral administration of Phellinus baumii (Agaricomycetes) extract. Int J Med Mushrooms 18:793-806

Sakakibara Y, Yanagisawa K, Katafuchi J, Ringer DP, Takami Y, Nakayama T, Suiko M, Liu MC (1998) Molecular cloning, expression, and characterization of novel human SULT1C sulfotransferases that catalyze the sulfonation of $N$-hydroxy2-acetylaminofluorene. J Biol Chem 273:33929-33935

Samuelsen JT, Holme JA, Låg M, Schwarze PE, Dahl JE, Becher R (2012) Biotransformation enzymes and lung cell response to 2-hydroxyethyl-methacrylate. J Biomed Mater Res A 100:462469. https://doi.org/10.1002/jbm.a.33287

Sarangapani R, Teeguarden JG, Cruzan G, Clewell HJ, Andersen ME (2002) Physiologically based pharmacokinetic modeling of styrene and styrene oxide respiratory-tract dosimetry in rodents and humans. Inhal Toxicol 14:789-834

Schladt L, Wörner W, Setiabudi F, Oesch F (1986) Distribution and inducibility of cytosolic epoxide hydrolase in male SpragueDawley rats. Biochem Pharmacol 35:3309-3316

Schlicht KE, Berg JZ, Murphy SE (2009) Effect of CYP2A13 active site mutation N297A on metabolism of coumarin and tobaccospecific nitrosamines. Drug Metab Dispos 37:665-671

Schmalix WA, Barrenscheen M, Landsiedel R, Janzowski C, Eisenbrand G, Gonzalez F, Eliasson E, Ingelman-Sundberg M, Perchermeier M, Greim H, Doehmer J (1995) Stable expression of human cytochrome P450 2E1 in V79 Chinese hamster cells. Eur J Pharmacol EnvironToxicol Pharmacol Sect 293:123-131

Schmassmann H, Sparrow A, Platt K, Oesch F (1978) Epoxide hydratase and benzo(a)pyrene monooxygenase activities in liver, kidney and lung after treatment of rats with epoxides of widely varying structures. Biochem Pharmacol 27:2237-2245

Seaton MJ, Plopper CG, Bond JA (1996) 1,3-Butadiene metabolism by lung airways isolated from mice and rats. Toxicology 113:314-317

Serabjit-Singh CJ, Devereux TR, Fouts JR, Philpot RM, Plopper CG (1980) Rabbit pulmonary monooxygenase enzymes in tissue sections and in isolated cell fractions. In: Gustafsson JA, CarlstedtDuke J, Mode A, Rafter J (eds) Biochemistry, biophysics and regulation of cytochrome P-450. Elsevier, New York, pp 451-454

Serabjit-Singh CJ, Nishio SJ, Philpot RM, Plopper CG (1988) The distribution of cytochrome P-450 monooxygenase in cells of the rabbit lung: an ultrastructural immunocytochemical characterization. Mol Pharmacol 33:279-289 
Sheets PL, Yost GS, Carlson GP (2004) Benzene metabolism in human lung cell lines BEAS-2B and A549 and cells overexpressing CYP2F1. J Biochem Mol Toxicol 18:92-99

Shen S, Li L, Ding X, Zheng J (2014) Metabolism of styrene to styrene oxide and vinylphenols in cytochrome P450 2F2- and P450 2E1-knockout mouse liver and lung microsomes. Chem Res Toxicol 27(1):27-33. https://doi.org/10.1021/tx400305w

Shimada T, Martin MV, Pruess-Schwartz D, Marnett LJ, Guengerich FP (1989) Roles of individual human cytochrome P-450 enzymes in the bioactivation of benzo(a)pyrene, 7,8-dihydroxy7,8-dihydrobenzo(a)pyrene, and other dihydrodiol derivatives of polycyclic aromatic hydrocarbons. Cancer Res 49:6304-6312

Shimada T, Yun C-H, Yamazaki H, Gautier J-C, Beaune PH, Guengerich FP (1992) Characterization of human lung microsomal cytochrome P-450 1A1 and its role in the oxidation of chemical carcinogens. Mol Pharmacol 41:856-864

Shimada T, Hayes CL, Yamazaki H, Amin S, Hecht SS, Guengerich FP, Sutter TR (1996a) Activation of chemically diverse procarcinogens by human cytochrome P-450 1B1. Cancer Res 56:2979-2984

Shimada T, Yamazaki H, Mimura M, Wakamiya N, Ueng YF et al (1996b) Characterization of microsomal cytochrome P450 enzymes involved in the oxidation of xenobiotic chemicals in human fetal liver and adult lungs. Drug Metab Dispos 24:515-522

Shultz MA, Choudary PV, Buckpitt AR (1999) Role of murine cytochrome P-4502F2 in metabolic activation of naphthalene and metabolism of other xenobiotics. J Pharmacol Exp Ther 290:281-288

Shultz MA, Morin D, Chang AM, Buckpitt A (2001) Metabolic capabilities of CYP2F2 with various pulmonary toxicants and its relative abundance in mouse lung subcompartments. J Pharmacol Exp Ther 296:510-519

Simmonds AC, Reilly CA, Baldwin RM, Ghanayem BI, Lanza DL, Yost GS, Collins KS, Forkert PG (2004) Bioactivation of 1,1-dichloroethylene to its epoxide by CYP2E1 and CYP2F enzymes. Drug Metab Dispos 32:1032-1039

Sivars KB, Sivars U, Hornberg E, Zhang H, Brändén L, Bonfante R, Huang S, Constant S, Robinson I, Betts CJ, Åberg PM (2018) A $3 \mathrm{~d}$ human airway model enables prediction of respiratory toxicity of inhaled drugs in vitro. Toxicol Sci 162:301-308

Sivils JC, Ancrum TM, Bain LJ (2013) Loss of Mrp1 alters detoxification enzyme expression in a tissue- and hormonal-status-specific manner. J Appl Toxicol 33:766-773. https://doi.org/10.1002/ jat. 2727

Slaughter SR, Statham CN, Philpot RM, Boyd MR (1983) Covalent binding of metabolites of 4-ipomeanol to rabbit pulmonary and hepatic microsomal proteins and to the enzymes of the pulmonary cytochrome P-450-dependent monooxygenase system. J Pharmacol Exp Ther 224:252-257

Slaus K, Coughtrie MW, Sharp S, Vanhaecke T, Vercruysse A, Rogiers V (2001) Influence of culture system and medium enrichment on sulfotransferase and sulfatase expression in male rat hepatocyte cultures. Biochem Pharmacol 61:1107-1117

Smith BR, Brian WR (1991) The role of metabolism in chemicalinduced pulmonary toxicity. Toxicol Pathol 19(4 Pt 1):470-481

Smith BR, Plummer JL, Wolf CR, Philpot RM, Bend JR (1982) p-Xylene metabolism by rabbit lung and liver and its relationship to the selective destruction of pulmonary cytochrome P-450. J Pharmacol Exp Ther 223:736-742

Smith TJ, Stoner GD, Yang CS (1995) Activation of 4-(methylnitrosamino)-1-(3-pyridyl)-1-butanone (NNK) in human lung microsomes by cytochromes $\mathrm{P} 450$, lipoxygenase, and hydroperoxides. Cancer Res 55:5566-5573

Smith KR, Pinkerton KE, Watanabe T, Pedersen TL, Ma SJ, Hammock BD (2005) Attenuation of tobacco smoke-induced lung inflammation by treatment with a soluble epoxide hydrolase inhibitor. Proc Natl Acad Sci USA 102:2186-2191

Somers GI, Lindsay N, Lowdon BM, Jones AE, Freathy C, Ho S, Woodrooffe AJ, Bayliss MK, Manchee GR (2007) A comparison of the expression and metabolizing activities of phase I and II enzymes in freshly isolated human lung parenchymal cells and cryopreserved human hepatocytes. Drug Metab Dispos 35:1797-1805

Spivack SD, Hurteau GJ, Reilly AA, Aldous KM, Ding X, Kaminsky LS (2001) CYP1B1 expression in human lung. Drug Metab Dispos 29:916-922

Stoddard EG, Volk RF, Carson JP, Ljungberg CM, Murphree TA, Smith JN, Sadler NC, Shukla AK, Ansong C, Wright AT (2018) Multifunctional activity-based protein profiling of the developing lung. J Proteome Res 17:2623-2634. https://doi.org/10.1021/acs. jproteome.8b00086

Stone KC, Mercer RR, Gehr P, Stockstill B, Crapo JD (1992) Allometric relationships of cell numbers and size in the mammalian lung. Am J Respir Cell Mol Biol 6:235-243

Stucki AO, Stucki JD, Hall SR et al (2015) A lung-on-a-chip array with an integrated bio-inspired respiration mechanism. Lab Chip 15:1302-1310. https://doi.org/10.1039/c4lc01252f

Sturton RG, Trifilieff A, Nicholson AG et al (2008) Pharmacological characterization of indacaterol, a novel once daily inhaled $\beta 2$ adrenoceptor agonist, on small airways in human and rat precision-cut lung slices. J Pharmacol Exp Ther 324:270-275. https ://doi.org/10.1124/jpet.107.129296

Su T, Bao ZP, Zhang QY, Smith TJ, Hong JY, Ding X (2000) Human cytochrome P450 CYP2A13: predominant expression in the respiratory tract and its high efficiency metabolic activation of a tobacco-specific carcinogen, 4-(methylnitrosamino)-1-(3pyridyl)-1-butanone. Cancer Res 60:5074-5079

Sukhanov VA, Sotnichenko AI, Serdyuk OA, Kalinina EV, Fedorova TY, Saprin AN, Piruzyan LA (2009) Influence of seasonal fluctuations, sex, and age on epoxide synthetase and epoxide hydrolase activities of the xenobiotic-metabolizing enzyme system. Dokl Biol Sci. 429:500-502

Sukumaran S, Jusko WJ, Dubois DC, Almon RR (2011) Light-dark oscillations in the lung transcriptome: implications for lung homeostasis, repair, metabolism, disease, and drug action. J Appl Physiol 110:1732-1747. https://doi.org/10.1152/japplphysi ol.00079.2011

Sumner SJ, Fennell TR (1994) Review of the metabolic fate of styrene. Crit Rev Toxicol 24(S1):S11-S33

Sutter TR, Tang YM, Hayes CL, Wo YY, Jabs EW, Li X, Yin H, Cody CW, Greenlee WF (1994) Complete cDNA sequence of a human dioxin-inducible mRNA identifies a new gene subfamily of cytochrome $\mathrm{P} 450$ that maps to chromosome 2. J Biol Chem 6(269):13092-13099

Swain RJ, Kemp SJ, Goldstraw P et al (2008) Spectral monitoring of surfactant clearance during alveolar epithelial Type II cell differentiation. Biophys J 95:5978-5987. https://doi.org/10.1529/ biophysj.108.136168

Tablin F, den Hartigh LJ, Aung HH, Lame MW, Kleeman MJ, Ham W, Norris JW, Pombo M, Wilson DW (2012) Seasonal influences on CAPs exposures: differential responses in platelet activation, serum cytokines and xenobiotic gene expression. Inhal Toxicol. 24:506-517. https://doi.org/10.3109/08958378.2012.695815

Takahashi K, Mitsui M, Takeuchi K et al (2004) Preservation of the characteristics of the cultured human Type II alveolar epithelial cells. Lung 182:213-226. https://doi.org/10.1007/s0040 8-004-2504-5

Takahashi K, Pavlidis S, Ng Kee Kwong F, Hoda U, Rossios C, Sun K, Loza M, Baribaud F, Chanez P, Fowler SJ, Horvath I, Montuschi P, Singer F, Musial J, Dahlen B, Dahlen SE, Krug N, Sandstrom T, Shaw DE, Lutter R, Bakke P, Fleming LJ, Howarth 
PH, Caruso M, Sousa AR, Corfield J, Auffray C, De Meulder B, Lefaudeux D, Djukanovic R, Sterk PJ, Guo Y, Adcock IM, Chung KF (2018) Sputum proteomics and airway cell transcripts of current and ex-smokers with severe asthma in U-BIOPRED: an exploratory analysis. Eur Respir J 51(5):1702173. https://doi. org/10.1183/13993003.02173-2017 (Print 2018 May)

Tang YM, Chen GF, Thompson PA et al (1999) Development of an antipeptide antibody that binds to the C-terminal region of human CYP1B1. Drug Metab Dispos 27:274-280

Tang SC, Sheu GT, Wong RH et al (2010) Expression of glutathione $S$-transferase M2 in stage I/II non-small cell lung cancer and alleviation of DNA damage exposure to benzo[a]pyrene. Toxicol Lett 192:316-323

Tanimura N, Kusunose N, Matsunaga N, Koyanagi S, Ohdo S (2011) Aryl hydrocarbon receptor-mediated Cyp1a1 expression is modulated in a CLOCK-dependent circadian manner. Toxicology 290:203-207. https://doi.org/10.1016/j.tox.2011.09.007

Theken KN, Deng Y, Kannon MA et al (2011) Activation of the acute inflammatory response alters cytochrome $\mathrm{P} 450$ expression and eicosanoid metabolism. Drug Metab Dispos 39:22-29

Thornton-Manning J, Appleton ML, Gonzalez FJ, Yost GS (1996) Metabolism of 3-methylindole by vaccinia-expressed P450 enzymes: correlation of 3-methyleneindolenine formation and protein-binding. J Pharmacol Exp Ther 276:21-29

Thum T, Erpenbeck VJ, Moeller J, Hohlfeld JM, Krug N, Borlak J (2006) Environ Health Perspect 114:1655-1661

Toussaint C, Albin N, Massaad L, Grunenwald D, Parise O Jr, Morizet J, Gouyette A, Chabot GG (1993) Main drug- and carcinogenmetabolizing enzyme systems in human non-small cell lung cancer and peritumoral tissues. Cancer Res 53:4608-4612

Tsuchiya Y, Nakajima M, Itoh S et al (2003) Expression of aryl hydrocarbon receptor repressor in normal human tissues and inducibility by polycyclic aromatic hydrocarbons in human tumor-derived cell lines. Toxicol Sci 72:253-259

Turgeon D, Carrier JS, Lévesque E, Hum DW, Bélanger A (2001) Relative enzymatic activity, protein stability, and tissue distribution of human steroid-metabolizing UGT2B subfamily members. Endocrinology 142:778-787

U. S. NCI, U.S. National Cancer Institute (1979) Bioassay of a solution of beta-nitrostyrene and styrene for possible carcinogenicity. NCI-CG-TR-170. Bethesda, MD: U.S. Department of Health, Education, and Welfare, Public Health Service, National Institutes of Health

Uehara S, Uno Y, Oshio T, Inoue T, Sasaki E, Yamazaki H (2018) Marmoset pulmonary cytochrome P450 2F1 oxidizes biphenyl and 7-ethoxycoumarin and hepatic human P450 substrates. Xenobiotica 48:656-662. https://doi.org/10.1080/00498254.2017.1354138

Ueng TH, Hu SH, Chen RM, Wang HW, Kuo ML (2000) Induction of cytochrome P-450 1A1 in human hepatoma HepG2 and lung carcinoma NCI-H322 cells by motorcycle exhaust particulate. $\mathbf{J}$ Toxicol Environ Health 60:101-119

Umachandran M, Ioannides C (2006) Stability of cytochromes P450 and phase II conjugation systems in precision-cut rat lung slices cultured up to $72 \mathrm{~h}$. Toxicology 224:14-21

Umachandran M, Howarth J, Ioannides C (2004) Metabolic and structural viability of precision-cut rat lung slices in culture. Xenobiotica 34:771-780

van Bladeren PJ, Sayer JM, Ryan DE, Thomas PE, Levin W, Jerina DM (1985) Differential stereoselectivity of cytochromes P-450b and P-450c in the formation of naphthalene and anthracene 1,2-oxides. The role of epoxide hydrolase in determining the enantiomer composition of the 1,2- dihydrodiols formed. J Biol Chem 260:10226-10235

van Duinen V, Trietsch SJ, Joore J, Vulto P, Hankemeier T (2015) Microfluidic 3D cell culture: from tools to tissue models. Curr Opin Biotechnol 35:118-126
Van Dyck E, Nazarov PV, Muller A, Nicot N, Bosseler M, Pierson S, Van Moer K, Palissot V, Mascaux C, Knolle U, Ninane V, Nati R, Bremnes RM, Vallar L, Berchem G, Schlesser M (2014) Bronchial airway gene expression in smokers with lung or head and neck cancer. Cancer Med 3:322-336. https://doi.org/10.1002/ cam4.190

Van Haute L, De Block G, Liebaers I, Sermon K, De Rycke M (2009) Generation of lung epithelial-like tissue from human embryonic stem cells. Respir Res 10:105. https://doi. org/10.1186/1465-9921-10-105

Van Vleet TR, Klein PJ, Coulombe RA (2001) Metabolism of aflatoxin B1 by normal human bronchial cells. J Toxicol Environ Health A 63:525-540

Vanderslice RR, Domin BA, Carver GT, Philpot RM (1987) Speciesdependent expression and induction of homologues of rabbit cytochrome P-450 isozyme 5 in liver and lung. Mol Pharmacol $31: 320-325$

Vanhees K, van Schooten FJ, Moonen EJ, Maas LM, van Waalwijk van Doorn-Khosrovani SB, Godschalk RW (2012) Maternal intake of quercetin during gestation alters ex vivo benzo[a]pyrene metabolism and DNA adduct formation in adult offspring. Mutagenesis 27:445-451. https://doi.org/10.1093/mutage/ges002

Vaughan MB, Ramirez RD, Wright WE et al (2006) A three-dimensional model of differentiation of immortalized human bronchial epithelial cells. Differentiation 74:141-148. https://doi.org/10.1 111/j.1432-0436.2006.00069.x

Veith A, Moorthy B (2018) Role of cytochrome P450 s in the generation and metabolism of reactive oxygen species. Curr Opin Toxicol 7:44-51. https://doi.org/10.1016/j.cotox.10.003

Verschoyle RD, Philpot RM, Wolf CR, Dinsdale D (1993) CYP4B1 activates 4-ipomeanol in rat lung. Toxicol Appl Pharmacol 123:193-198

Vickers AE, Jimenez RM, Spaans MC, Pflimlin V, Fisher RL, Brendel K (1997) Human and rat lung biotransformation of cyclosporin A and its derivatives using slices and bronchial epithelial cells. Drug Metab Dispos 25:873-880

Vieira I, Passanen M, Raunio H, Cresteil T (1998) Expression of CYP2E1 in human lung and kidney during development and in full-term placenta: a differential methylation of the gene is involved in the regulation process. Pharmacol Toxicol 83:183-187

Visser M, Weber K, Rincon G, Merritt D (2017) Use of RNA-seq to determine variation in canine cytochrome P450 mRNA expression between blood, liver, lung, kidney and duodenum in healthy beagles. J Vet Pharmacol Ther 40:583-590. https:// doi.org/10.1111/jvp. 12400

Vodicka P, Koskinen M, Naccarati A, Oesch-Bartlomowicz B, Vodickova L, Hemminki K, Oesch F (2006) Styrene metabolism, genotoxicity, and potential carcinogenicity. Drug Metab Rev 38:805-853

Vogel-Bindel U, Bentley P, Oesch F (1982) Endogenous role of microsomal epoxide hydrolase. Ontogenesis, induction inhibition, tissue distribution, immunological behaviour and purification of microsomal epoxide hydrolase with 16 alpha, 17 alpha-epoxyandrostene-3-one as substrate. Eur J Biochem 126:425-431

Walraven JM, Barker DF, Doll MA, Hein DW (2007) Tissue expression and genomic sequences of rat $\mathrm{N}$-acetyltransferases rNat1, rNat2, rNat 3 , and Functional characterization of a novel rNat $3 * 2$ genetic variant. Toxicol Sci 99:413-421

Walters M, Gomi K, Ashbridge B et al (2013) Generation of a human airway epithelium derived basal cell line with multipotent differentiation capacity. Respir Res 14:135. https://doi. org/10.1186/1465-9921-14-135

Walters MS, Salit J, Ju JH, Staudt MR, Kaner RJ, Rogalski AM, Sodeinde TB, Rahim R, Strulovici-Barel Y, Mezey JG, Almulla 
AM, Sattar H, Mahmoud M, Crystal RG (2017) Waterpipe smoking induces epigenetic changes in the small airway epithelium. PLoS One 12:e0171112. https://doi.org/10.1371/journ al.pone.0171112 (eCollection 2017)

Wan J, Carr BA, Cutler NS, Lanza DL, Hines RN, Yost GS (2005) Drug Metab Dispos 33:1244-1253

Wang H, Lanza DL, Yost GS (1998) Cloning and expression of CYP2F3, a cytochrome P450 that bioactivates the selective pneumotoxins 3-methylindole and naphthalene. Arch Biochem Biophys 349:329-340

Wang Y, Spitz MR, Schabath MB et al (2003) Association between glutathione $S$-transferase P1 polymorphisms and lung cancer risk in Caucasians: a case-control study. Lung Cancer 40:25-32

Wang D, Haviland DL, Burns AR, Zsigmond E, Wetsel RA (2007) A pure population of lung alveolar epithelial type II cells derived from human embryonic stem cells. Proc Natl Acad Sci USA 104:4449-4454

Wang P, Zheng J, Qu L, Lian L, Xu G, Yang N (2013) Molecular cloning, sequence characterization, SNP detection, and tissue expression analysis of duck FMO3 gene. Mol Cell Biochem 379:141-151. https://doi.org/10.1007/s11010-013-1636-4

Warren DL, Brown DL, Buckpitt AR (1982) Evidence for cytochrome P-450 mediated metabolism in the bronchiolar damage by naphthalene. Chem Biol Interact 40:287-303

Watt KC, Buckpitt AR (2000) Species differences in the regio- and stereoselectivity of 1-nitronaphthalene metabolism. Drug Metab Dispos 28:376-378

Wattenberg LW, Leong JL (1962) Histochemical demonstration of reduced pyridine nucleotide dependent polycyclic hydrocarbon metabolizing systems. J Histochem Cytochem 10:412-420

Waxman DJ (1999) P450 gene induction by structurally diverse xenochemicals: central role of nuclear receptors CAR, PXR, and PPAR. Arch Biochem Biophys 369:11-23

Waxman DJ, Lapenson DP, Aoyama T, Gelboin HV, Gonzalez FJ, Korzekwa K (1991) Steroid hormone hydroxylase specificities of eleven cDNA-expressed human cytochrome P450s. Arch Biochem Biophys 290:160-166

Weems JM, Yost GS (2010) 3-Methylindole metabolites induce lung CYP1A1 and CYP2F1 enzymes by AhR and non-AhR mechanisms, respectively. Chem Res Toxicol 23:696-704. https://doi. org/10.1021/tx9004506

Wei C, Caccavale RJ, Weyand EH, Chen S, Iba MM (2002) Induction of CYP1A1 and CYP1A2 expressions by prototypic and atypical inducers in the human lung. Cancer Lett 178:25-36

Wei Y, Wu H, Li L, Liu Z, Zhou X, Zhang QY, Weng Y, D’Agostino J, Ling G, Zhang X, Kluetzman K, Yao Y, Ding X (2012) Generation and characterization of a CYP2A13/2B6/2F1-transgenic mouse model. Drug Metab Dispos 40:1144-1150. https://doi. org/10.1124/dmd.112.044826

Weng Y, Fang C, Turesky RJ, Behr M, Kaminsky LS, Ding X (2007) Determination of the role of target tissue metabolism in lung carcinogenesis using conditional cytochrome $\mathrm{P} 450$ reductasenull mice. Cancer Res 67:7825-7832

Wenker MAM, Kecic S, Monster AC, de Wolff FA (2001) Metabolism of styrene in human liver in vitro: interindividual variation and enantioselectivity. Xenobiotica 31:61-72

Wermuth B, Platt KL, Seidel A, Oesch F (1986) Carbonyl reductase provides the enzymatic basis of quinone detoxication in man. Biochem Pharmacol 35:1277-1282

Westlind A, Malmebo S, Johansson I, Otter C, Andersson TB, Ingelman-Sundberg M, Oscarson M (2001) Cloning and tissue distribution of a novel human cytochrome P450 of the CYP3A subfamily, CYP3A43. Biochem Biophys Res Commun 281:1349-1355
Wheeler CW, Guenthner TM (1991) Cytochrome P-450-dependent metabolism of xenobiotics in human lung. J Biochem Toxicol 6:163-169

Wheeler CW, Park SS, Guenthner TM (1990) Immunochemical analysis of cytochrome P-450IA1 homologue in human lung microsomes. Mol Pharmacol 38:634-643

Wheeler CW, Wrighton SA, Guenthner TM (1992) Detection of human lung cytochromes $\mathrm{P} 450$ that are immunochemically related to cytochrome P450IIE1 and cytochrome P450IIIA. Biochem Pharmacol 44:183-186

Willey JC, Coy E, Brolly C, Utell MJ, Frampton MW, Hammersley J, Thilly WG, Olson D, Cairns K (1996) Xenobiotic metabolism enzyme gene expression in human bronchial epithelial and alveolar macrophage cells. Am J Respir Cell Mol Biol 14:262-271

Williams DE, Hale SE, Okita RT, Masters BS (1984) A prostaglandin omega-hydroxylase cytochrome P-450 (P-450PGomega) purified from lungs of pregnant rabbits. J Biol Chem 259:14600-14608

Wilson AS, Davis CD, Williams DP, Buckpitt AR, Pirmohamed M, Park BK (1996) Characterisation of the toxic metabolite(s) of naphthalene. Toxicology 114:233-242 (Erratum in: Toxicology 1997 120:75)

Woenckhaus M, Klein-Hitpass L, Grepmeier U, Merk J, Pfeifer M, Wild P, Bettstetter M, Wuensch P, Blaszyk H, Hartmann A, Hofstaedter F, Dietmaier W (2006) Smoking and cancer-related gene expression in bronchial epithelium and non-small-cell lung cancers. J Pathol 210:192-204

Wolf CR, Szutowski MM, Ball LM, Philpot RM (1978) The rabbit pulmonary monooxygenase system: characteristics and activities of two forms of pulmonary cytochrome P-450. Chem Biol Interact 21:29-43

Wu X, Peters-Hall JR, Bose S et al (2011) Human bronchial epithelial cells differentiate to 3D glandular acini on basement membrane matrix. Am J Respir Cell Mol Biol 44:914-921. https://doi. org/10.1165/rcmb.2009-03290C

Würthwein G, Rohdewald P (1990) Activation of beclomethasone dipropionate by hydrolysis to beclomethasone-17-monopropionate. Biopharm Drug Dispos 11:381-394

Xiang C, Wang J, Kou X, Chen X, Qin Z, Jiang Y, Sun C, Xu J, Tan W, Jin L, Lin D, He F, Wang H (2015) Pulmonary expression of CYP2A13 and ABCB1 is regulated by FOXA2, and their genetic interaction is associated with lung cancer. FASEB J 29:19861998. https://doi.org/10.1096/fj.14-264580

Yilmaz Y, Umehara K, Williams G, Faller T, Schiller H, Walles M, Kraehenbuehl S, Camenisch G, Manevski N (2018) Assessment of the pulmonary CYP1A1 metabolism of mavoglurant (AFQ056) in rat. Xenobiotica 48:793-803. https://doi. org/10.1080/00498254.2017.1373311

Yilmaz Y, Williams G, Walles M, Manevski N, Krahenbuhl S, Camenisch G (2019) Comparison of rat and human pulmonary metabolism using precision-cut lung slices (PCLS). Drug Metab Lett 13:53-63. https://doi.org/10.2174/18723128126661810221 14622

Yokose T, Doy M, Taniguchi T, Shimada T, Kakiki M, Horie T, Matsuzaki Y, Mukai K (1999) Immunohistochemical study of cytochrome P450 2C and 3A in human non-neoplastic and neoplastic tissues. Virchows Arch 434:401-411

Yokota H, Kunimasa Y, Shimoyama Y, Kobayashi T, Matsumoto J, Yuasa A (2002) Effects on extrahepatic UDP-glucuronosyltransferases in hypophysectomized rat. J Biochem 132:265-270

Yoon M, Madden MC, Barton HA (2006) Developmental expression of aldehyde dehydrogenase in rat: a comparison of liver and lung development. Toxicol Sci 89:386-398

Yun JH, Morrow J, Owen CA, Qiu W, Glass K, Lao T, Jiang Z, Perrella MA, Silverman EK, Zhou X, Hersh CP (2017) Transcriptomic analysis of lung tissue from cigarette smoke-induced emphysema 
murine models and human chronic obstructive pulmonary disease show shared and distinct pathways. Am J Respir Cell Mol Biol. 57:47-58. https://doi.org/10.1165/rcmb.2016-0328OC

Zeldin DC, Plitman JD, Kobayashi J, Miller RF, Snapper JR, Falck JR et al (1995) The rabbit pulmonary cytochrome P450 arachidonic acid metabolic pathway: characterization and significance. J Clin Invest 95:2150-2160

Zeldin DC, Foley J, Ma J, Boyle JE, Pascual JM, Moomaw CR, Tomer KB, Steenbergen C, Wu S (1996) CYP2J subfamily P450 s in the lung: expression, localization and potential functional significance. Mol Pharmacol 50:1111-1117

Zhang J, Cashman JR (2006) Quantitative analysis of FMO gene mRNA levels in human tissues. Drug Metab Dispos 34:19-26

Zhang JY, Wang Y, Prakash C (2006) Xenobiotic-metabolizing enzymes in human lung. Curr Drug Metab 7:939-948

Zhang H, Liu H, Davies KJ, Sioutas C, Finch CE, Morgan TE, Forman HJ (2012) Nrf2-regulated phase II enzymes are induced by chronic ambient nanoparticle exposure in young mice with agerelated impairments. Free Radic Biol Med 52:2038-2346. https ://doi.org/10.1016/j.freeradbiomed.2012.02.042

Zheng YM, Fisher MB, Yokotani N, Fujii-Kuriyama Y, Rettie AE (1998) Identification of a meander region proline residue critical for heme binding to cytochrome P450: implications for the catalytic function of human CYP4B1. Biochemistry 37:12847-12851

Zheng J, Plopper CG, Lakritz J, Storms DH, Hammock BD (2001) Leukotoxin-diol: a putative toxic mediator involved in acute respiratory distress syndrome. Am J Respir Cell Mol Biol 5(4):434-438

Zheng Z, Fang JL, Lazarus P (2002) Glucuronidation: an important mechanism for detoxification of benzo[a]pyrene metabolites in aerodigestive tract tissues. Drug Metab Dispos 30:397-403
Zheng R, Dragomir AC, Mishin V, Richardson JR, Heck DE, Laskin DL, Laskin JD (2014) Differential metabolism of 4-hydroxynonenal in liver, lung and brain of mice and rats. Toxicol Appl Pharmacol 279:43-52. https://doi.org/10.1016/j.taap.2014.04.026

Zhou X, D'Agostino J, Li L, Moore CD, Yost GS, Ding X (2012) Respective roles of CYP2A5 and CYP2F2 in the bioactivation of 3-methylindole in mouse olfactory mucosa and lung: studies using Cyp2a5-null and Cyp2f2-null mouse models. Drug Metab Dispos 40:642-647. https://doi.org/10.1124/dmd.111.044081

Zhu D, Zhang C, Medhora M, Jacobs ER (1985) CYP4A mRNA, protein, and product in rat lungs: novel localization in vascular endothelium. J Appl Physiol 93:330-337

Zhu LR, Thomas PE, Lu G et al (2006) CYP2A13 in human respiratory tissues and lung cancers: an immunohistochemical study with a new peptide-specific antibody. Drug Metab Dispos 34:1672-1676

Zuo WL, Shenoy SA, Li S, O’Beirne SL, Strulovici-Barel Y, Leopold PL, Wang G, Staudt MR, Walters MS, Mason C, Kaner RJ, Mezey JG, Crystal RG (2018) Ontogeny and biology of human small airway epithelial club cells. Am J Respir Crit Care Med 198:1375-1388. https://doi.org/10.1164/rccm.201710-2107OC

Publisher's Note Springer Nature remains neutral with regard to jurisdictional claims in published maps and institutional affiliations. 